\title{
Closed Orbit Feedback System for the Fast Ramping Hadron Synchrotrons
}

\author{
DISSERTATION \\ zur Erlangung des Doktrogrades \\ der Naturwissenschaften
}

vergelegt am Fachbereich Physik

der Johann Wolfgang Goethe-Universität

in Frankfurt am Main
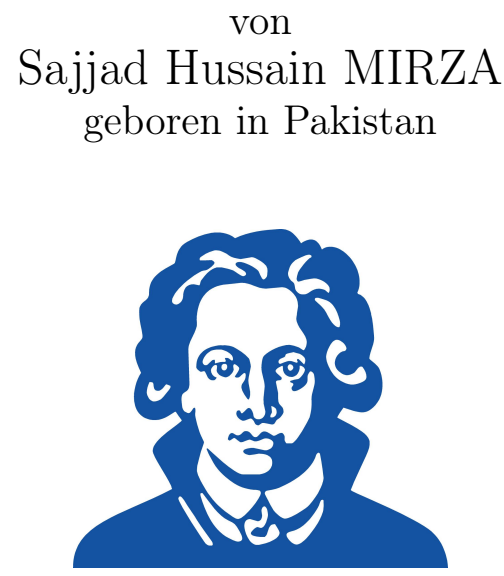

Frankfurt am Main 2021

(D30) 
vom Fachbereich Physik der

Johann Wolfgang Goethe-Universität als Dissertation angenommen.

Datum der Disputation: 11 May 2021.

\section{Dekan:}

Prof. Dr. Harald Appelshäuser

\section{Gutachter:}

PD Dr. Giuliano Franchetti

Prof. Dr. Ulrich Ratzinger 


\section{Abstract}

The realization of a fast and robust closed orbit feedback (COFB) system for the on-ramp

orbit correction at SIS18 synchrotron of FAIR project is reported in this thesis. SIS18 has some peculiar behaviors including on-ramp optics variation, very short lengths of the ramps (200 ms to $1 \mathrm{~s}$ ) and a cycle-to-cycle variation of beam parameters. The realized fast COFB system being robust against above mentioned features of SIS18 is a first of its kind and the course to its realization led to some novel contributions in the field of closed orbit correction. A new method relying on the discrete Fourier transform (DFT)based decomposition of the orbit response matrix (ORM) has been introduced, exploiting the symmetry in the arrangement of beam position monitors (BPMs) and the corrector magnets in the synchrotrons. A nearest-circulant approximation has also been introduced for synchrotrons having slight deviation from the symmetry, making the method applicable to a vast majority of synchrotrons. Moreover, the performance and the stability analysis of COFB systems in the presence of ORM mismatch between the synchrotron and the feedback controller is presented. The COFB systems are divided into slow and fast regimes and a new stability criterion consistent with measurements, is introduced. The practicality of the criterion is verified experimentally at COSY Jülich and is used for the analysis of various sources of ORM mismatch at SIS18. The commissioning of the SIS18 COFB system is also reported in detail which relies on Libera Hadron as the main hardware resource for the controller implementation. The on-ramp orbit correction is demonstrated for the horizontal plane of SIS18, for the disturbance rejection up to $600 \mathrm{~Hz}$. 


\section{Dedication}

I dedicate my dissertation to my mother and my late father, for their countless efforts behind every success of my life. 


\section{Zusammenfassung}

Thema dieser Doktorarbeit ist die Entwicklung eines Systems zur schnellen Orbitkorrektur (Englisch: closed orbit feedback, COFB) Systems und dessen Anwendung an den GSISchwerionensynchrotrons SIS18 bzw. SIS100. Das wichtigste Ziel ist es eine Ortsstabilität (RMS-Wert) unterhalb $1 \mathrm{~mm}$ zu erreichen insbesondere auch während der Beschleunigungsrampe; diese Ortsstabilität entspricht ca. 10\% der RMS-Strahlbreite. Das COFB-System soll desweiteren möglichst breitbandig wirken können - die natürliche obere Grenzfrequenz ist hier durch die erreichbare Bandbreite der Korrektordipolmagnete gegeben. In SynchrotronLichtquellen und Hadronen-Beschleunigern sind COFB Systeme integraler Bestandteil eines ordnungsgemäßen Betriebs.

Die Korrekturen eines COFB-Systems basiert auf der Messung der transversalen Ortsablage an diskreten Stellen im Synchrotron und die Korrektur mit Hilfe von ebenfalls an diskreten Stellen installierten Korrektordipolmagneten. Die lineare Antwort zwischen der Wirkung der Korrekturmagnete auf die Ortsablagen wird durch die Orbit Response Matrix (ORM) beschrieben. Im Gegensatz zu den meisten Synchrotrons hat das SIS18 die Besonderheit, dass diese ORM während der Beschleunigung variiert, da die Fokussierung durch die QuadrupolMagnet verändert wird. Die maximale Bandbreite üblicher COFB-System liegt im Bereich vom maximal $150 \mathrm{~Hz}$, während hier eine höhere Bandbreite notwendig ist. Für den Fall von SIS18 sind starke Störungen durch die Netzgeräte Variation bei $150 \mathrm{~Hz}$ und ihren harmonischen Frequenzen vorhanden, die während der Beschleunigungsrampe (typische Dauer zwischen $200 \mathrm{~ms}$ und $1000 \mathrm{~ms}$ ) korrigiert werden müssen. Um nun die oben genannten Ziele zu erreichen, müssen folgende Fragen beantwortet werden:

- Welche Methode der Orbitkorrektur funktioniert am besten während der Beschleunigungungsrampe, bei der sich die Beschleunigeroptik und damit die ORM ändert?

- Wie häufig soll die zur Korrektur verwendete ORM während der Beschleunigungsrampe aktualisiert werden, damit die geforderte Ortsstabilität und Bandbreite durchgehalten werden kann?

- Falls aus technischen Gründen die ORM nicht aktualisiert werden kann, wie sind dann die festen oder variablen Regelparameter zu wählen?

- Unter welchen Bedingungen kann eine Instabilität der Rückkopplung vermieden werden?

- Welche Auswirkungen hat die strahlintensitätsabhängige Verschiebung des Arbeitspunktes (tune shift) auf die Stabilität des COFB-Systems? 
Die Antworten auf diese Frage sind ausschlaggebend für das Design und die Realisierung des COFB-Systems am SIS18 und wurden deshalb in dieser Arbeit ausführlich untersucht. Sie führten außerdem zu neuartigen Einsichten, die auch für andere Beschleuniger Anwendung finden können.

COFB-Systeme werden schon seit einigen Jahrzenten unter Verwendung verschiedener Methoden realisiert. In letzter Zeit wird meistens ein auf der Singulärwertzelegung (SVD) der ORM-basierendes Verfahren verwendet. Formal werden zur Orbitkorrektur die gemessenen Strahlpositionen ausgewertet und die entsprechenden Korrekturmagnetfeldstärken mithilfe der inversen ORM berechnet. Zur (Pseudo-)Invertierung ist hier das SVD-Verfahren günstig, die die reellwertige ORM in zwei orthogonale Matrizen $\mathbf{U}$ und $\mathbf{V}$ sowie eine Diagonalmatrix $\mathbf{S}$ zerlegt, so dass gilt ORM $=: \mathbf{R}=\mathbf{U S V}^{T}$. Die Einträge der Diagonalmatrix $\mathbf{S}$ heißen Singulärwerte. Die Pseudo-Inverse $\mathbf{R}^{+}$wird realisiert, indem man die Reziprokwerte derjenigen Singulärwerte durch Null ersetzt, die unterhalb einer gewissen Schelle liegen. Die Spalten der orthogonalen Matrizen sind eindeutig durch die Singulärwerte verkoppelt, so dass eine modenbasierte Rückkopplung durchführbar ist.

In dieser Arbeit wird das SVD-basierte Verfahren detailliert untersucht und die Vor- und Nachteile zusammengestellt. Z.B. müssen im Rahmen der SVD alle Matrizen (U, S und V) neu berechnet werden, wenn sich die Beschleunigeroptik und damit die ORM ändert, wie im Fall von SIS18 während der Beschleunigungsrampe vorkommt.

Ein auf diskreter Fouriertransformation (DFT) basierendes Verfahrenes der ORM-Zerlegung wurde in dieser Arbeit erstmalig detailliert beschrieben. Die Grundlage der Methode ist die periodische Anordnung der BPMs und Korrekturmagnete, die zu einer ORM mit sogenannter zyklischer bzw. blockzyklischer Symmetrie führt. Für das SIS18 ist die vertikale ORM ein Beispiel für eine zyklische Matrix, da jedes Segment einen BPM sowie einen Korrekturmagnet enthält und sich die Positionierung über die 12 Segmente wiederholt. In einer zyklischen bzw. blockzyklischen Matrix sind die Zeilen bzw. Spalten verbunden durch zyklische Permutation ihrer Elemente/Blöcke. Die DFT-Zerlegung der ORM führt auf ein Produkt dreier Matrizen $\mathbf{F} \mathbf{\Lambda} \mathbf{F}$, wobei $\mathbf{F}$ die Standardfouriermatrix beschreibt und die Diagonalmatrix $\boldsymbol{\Lambda}$ die Fourierkoeffizienten auf der Diagonalen entält. Für zyklische ORMs derselben Dimension ändert sich die Standardfouriermatrix nicht. Daher ist die Information der ORM in der Diagonalmatrix $\boldsymbol{\Lambda}$ konzentriert. Dies führt zu geringem Rechen- und Speicheraufwand, sobald die ORM aktualisiert werden soll (z.B. während der Beschleunigungsrampe). Weiterhin stellen die Spalten der Standardfouriermatrix Fouriermoden dar, die eine einfach physikalische Interpretation bzgl. verschiedener Strahlphänomene zulassen. Weiterhin kann damit der closed orbit zwischen den BPM Stationen interpoliert werden. Die Äquivalenz der DFT- und SVD-basierten Zerlegung zyklischer ORMs wurde in dieser Arbeit gezeigt.

Das DFT-basierte Verfahren wird in dieser Arbeit auch auf gebrochene Symmetrien bzgl. der BPM- und Korrekturmagnetanordnung angewandt durch die Einführung einer Approximation, die eine Matrix auf eine zyklische Matrix abbildet. Damit ist das DFT-basierte Verfahren für Synchtrotrons allgemein anwendbar, auch für Synchtrotron-Lichtquellen inklusive der Modifikationen der optischen Funktionen durch insertion devices. Die Verhältnisse für die horizontale Korrektur am SIS18 werden diskutiert, da dort die ORM durch die nicht-symmetrische Position zweier Korrekturmagnete gestört ist. Die Orbitkorrektur wird experimentell mit Hilfe der originalen ORM durchgeführt. Die Differenz der RMS-Werte des 
closed orbit zwischen der vollständigen ORM und der approximiert-zyklischen ORM ist nur ca. $9 \%$ und somit kann das DFT-basierte Verfahren auch für diesen Fall angewendet werden. Auch für SIS100 kann die blockzyklische Approximation der ORM benutzt werden, obwohl dort durch beta-beating die Symmetrie nicht ganz erfüllt ist.

In der Arbeit wird die Robustheit des DFT-basierten Verfahrens gegenüber Fehlmessungen der Strahlposition, sogenannter ,missing BPM` gezeigt, da die Strahlposition am Ort des fehlenden BPMs durch die erwähnte physikalische Interpretation der DFT-Moden rekonstruiert werden kann. Der Vorteil der DFT-basierten gegenüber der SVD-basierten Methode wird experimentell demonstriert durch die Manipulation der disperionsinduzierten Abweichungen vom closed orbit in der Horizontalebene des SIS18.

Ein zweiter wichtiger Inhalt dieser Dissertation ist die Modellierung des Verhaltens der ORM um deren Robustheit gegenüber Variationen des räumlichen Modells zwischen Synchrotron und Regler zu untersuchen. Damit kann ein Stabilitätskriterium angegeben werden um die Leistungsfähigkeit eines COFB-System zu beschreiben. Dies ist wichtig, da sich am SIS18 die ORM während der Beschleunigung ändert und somit eventuell die Reglerparameter angepasst werden müssen. Zur Beschreibung wird das COFB-System konzeptionell in zwei Bestandteile zerlegt: Dies ist einerseits der zeitabhängige Anteil der die zeitliche Reaktion der beteiligten Hardware und der Rückkoppelschleife angibt. Der zweite Teil betrifft den räumlichen Anteil, der mit Hilfe der ORM beschrieben wird. Somit kann nicht nur die Änderung während der Beschleunigung beschreiben werden, sondern auch Strahlstrom-abhängige Effekte wie die Verschiebung des Arbeitspunktes oder beta-beating Effekte.

Für eine zusätzliche Analyse wird das COFB-System konzeptionell zerlegt in ein sogenanntes schnelle und langsames Regime: In dem langsamen Regime sind die Zeitschritte der Orbitkorrektur mindestens eine Größenordnung langsamer als alle Zeitverzögerungen in dem elektronischen System (Systemverzögerung und offene Schleifenverstärkung, im folgenden offener Regelkreis genannt). Damit kann der zeitliche Anteil der Regelung zunächst vernachlässigt werden und die Stabilität ist nur durch das Verhalten der ORM zu charakterisieren. Die Korrektur-Matrix $\mathbf{M}=\mathbf{I}-\mathbf{R R}_{\Theta}^{+}$beschreibt die Abweichung zwischen der systemischen ORM $\mathbf{R}$ und der in der Steuerung benutzten ORM $\mathbf{R}_{\Theta}$. Die Stabilitätscharakterisierung erfolgt mit Hilfe des spektralen Radius $\rho(\mathbf{M})$; Stabilität wird erreicht falls $\rho(\mathbf{M})<1$ ist, was in dieser Arbeit erstmalig für ein COFB-System gezeigt wurde. Ein Vergleich mit dem üblicherweise verwendeten Kleinsignaltheorem wurde durchgeführt mit dem Ergebnis, dass unter Verwendung der Spektralradiusbedingung größere Toleranzbänder realisiert werden können im Vergleich zum Kleinsignaltheorem. Eine Relation des spektralen Radius zu der messbaren RMS Ortsabweichung nach einem Korrekturschritt $\delta_{1}$ wurde hergeleitet. Die Gültigkeit dieses Stabilitätskriterium wurde durch Simulationen sichergestellt und deren Richtigkeit durch strahlbasierte Messungen am COSY Speicherring beim FZ-Jülich durch Variation von $\rho(\mathbf{M})$ bestätigt.

Verschiedene Quellen der räumlichen Fehlanpassung für das SIS18 wurden simuliert wie z.B. die Variation der ORM während der Beschleunigungsrampe, intensitätsabhängige Arbeitspunktverschiebung und beta-beating. Der spektrale Radius wurde für diese Fälle berechnet: Beispielsweise ist für die ORM Variation während der Beschleunigungsrampe des SIS18 der maximale spektrale Radius 0.56 für die horizontale Ebene und 0.13 für die vertikale Ebene, wenn man statt der variierenden ORM ausschließlich die ORM bei Injektion benutzt. Für 
diesen Fall ist zu erwarten, dass das COFB System über die gesamte Beschleunigungsrampe stabil bleibt; allerdings unter signifikanter Einschränkung der Regelbandbreite. Für die intensitätsabhängige Arbeitspunktvariation wird ein Anstieg des spektralen Radius mit der Größe der Arbeitspunktverschiebung vorhergesagt, allerdings mit einer größeren Steigung in Abhängigkeit von der Änderung in Richtung eines ganzzahligen Arbeitspunkts verglichen mit einer entfernenden Bewegung. Das entsprechende Verhalten wurde für die Parameter am SIS18 simuliert und experimentell an COSY für ein langsames Regime bestätigt; dabei wurde das COFB durch Arbeitspunkt-verschiebende ORM-Werte in Richtung eines instabilen Bereichs geschoben. Weiterhin wurde gezeigt das beta-beating nicht zu Instabilitäten führt, so lange ein ganzzahligen Arbeitspunkt nicht erreicht wird.

Die Stabilitätsuntersuchungen wurden auch für das schnelle Regime unter Beachtung der System- und Rückkoppeldynamik durchgeführt. Die Transferfunktionen für den geschlossener Regelkreis werden als Funktionen von $\rho(\mathbf{M})$ hergeleitet. Auch hier kann der spektrale Radius als Stabilitätskriterium genutzt werden. Dabei zeigt sich erwartungsgemäß, dass die erreichbare Bandbreite der Korrekturen abnimmt bei steigenden $\rho(\mathbf{M})$. Benutzt man die Transfunktionen, so lassen sich die benötigten Aktualisierungsraten der ORM vorhersagen, um eine feste Bandbreite während des Rampens zu erreichen. Die geschlossener Regelkreis Instabilitäten entsprechen den rechtsseitigen Koordinaten der Polestellen der Transferfunktion innerhalb der komplexen Ebene (im mathematischen Sinne). Es wurde durch Simulationen gezeigt, dass die Pole bei steigenden $\rho(\mathbf{M})$ von der links- auf die rechtsseitige Ebene wandern. Die den Polestellen zugeordneten Frequenzen und die Instabilitätsschwellwert von $\rho(\mathbf{M})$ hängen von dem zeitlichen Verhalten des offener Regelkreis System ab und erreichen $\rho(\mathbf{M})=1$ im Fall vernachlässigbarer Systemverzögerung. Für den Fall $\rho(\mathbf{M})>1$ wird das COFBSystem auf jeden Fall instabil unabhängig von der Rückkopplungsdynamik. Die Skalierungen der Rückkoppelparameter als Funktion von $\rho(\mathbf{M})$ wurde hergeleitet und Schwellwerte der Instabilität bestimmt.

Das im Rahmen der Doktorarbeit entwickelte SIS18 COFB wurde mithilfe der Libera Hadron Hardware für die horizontale Ebene in Betrieb genommen. Die Libera Hadron Hardware wurde zur BPM Datenverarbeitung, Reglerimplementation und Berechnung der Korrekturmagnetfeldstärke benutzt. Dabei wurden 3 Liberas für die 12 im SIS18 installierten BPMs und 12 horizontalen Korrekturmagnete verwendet. Die System-Antwort hängt von den Subsystemen ab: Zunächst wurden die Charakteristik der Netzgeräte und deren Steuereinheit bestimmt, sowohl durch sinusartige Anregung bei fest Korrekturstrom (0.78 A) als auch mit Pulsen variabler Amplitude (0.78 bis 36 A mit Sollanstiegszeiten von $10 \mu \mathrm{s})$. Die Flankensteilheit (slew-rate dependence) wurde gemessen und führt zu einer Abnahme der Frequenz des dominierenden Pols für größere slew-rates des Eingangssignals. Die Charakterisierung des Gesamtsystems wurde zusätzlich durch strahlbasierte Messungen mit sinusartiger Anregung (0.78 A) durchgeführt. Dabei wurden neue Pole und Nullstellen im Bereich von 300 bis $400 \mathrm{~Hz}$ festgestellt, die sich für die einzelnen Netzgeräte unterscheiden. Die Parameter der proportional-integral Regelung (PI controller) der Libera Hardware Implementierung wurden mit Hilfe der Charakterisierung des Gesamtsystems bestimmt. Diese Werte sind nur eine erste Abschätzung, da sich auf Grund der Abhängigkeit von der slew-rate die Transfer-Funktion während der Beschleunigungsrampe ändert.

Die ORM wurde während der gesamten Beschleunigungsrampe am SIS18 für zwei unter- 
schiedliche Beschleunigereinstellungen vermessen, indem der Strahl sinusartig mit einer Amplitude von $1 \mathrm{mrad}$ angeregt wurde. Die räumliche Fehlanpassung (charakterisiert durch $\rho(\mathbf{M})$ ) wurde zu verschiedenen Zeitpunkten innerhalb der Beschleunigungsrampen in Beziehung zu MADX modellierten sowie gemessenen ORMs bei Injektion gesetzt. Das Maximum von $\rho(\mathbf{M})$ bei Extraktionsenergie ist vergleichbar mit dem zuvor berechneten Wert von 0.56 für die horizontale Ebene. Andererseits zeigte sich bei den strahlbasierten Messungen eine intrinsische Fehlanpassung zum MADX Model $(0.4<\rho(\mathbf{M})<0.6)$ schon bei Injektion sowie das Überschreiten des kritischen Werts von $\rho(\mathbf{M})>1$ während der Beschleunigungsrampe. Der Grund der Abweichung konnte nicht genau bestimmt werden und wird zukünftig noch zu untersuchen sein.

Am SIS18 wurde die Funktionalität des COFB-Systems demonstriert mit Hilfe der durch MADX berechneten ORM sowie unter Verwendung der gemessenen ORM bei Injektion, jeweils für konstante Regelparameter. Die maximal erreichte Bandbreite war etwa $600 \mathrm{~Hz}$ im Fall der gemessenen ORM (bei relativ kleinem Wert von $\rho(\mathbf{M})$ ) während im Fall der berechneten ORM (bei relativ großem Wert von $\rho(\mathbf{M})$ ) eine Bandbreite bis $300 \mathrm{~Hz}$ erreicht wurde. Dies bestätigt die zuvor beschriebenen theoretischen Überlegungen, nämlich dass räumliche Fehlanpassungen die maximale Bandbreite signifikant beeinflusst. Höhere Werte für die Regelparameter bei Benutzung der MADX ORM resultierten in einer Instabilität des closed orbit. Die Frequenz des instabilen Pols liegt bei $335 \mathrm{~Hz}$; ein Wert der nahe am Pol des offener Regelkreis Systems bei $322 \mathrm{~Hz}$ liegt, aber kleiner ist als der vorhergesagte Wert für das offener Regelkreis System. Dies kann durch die Verwendung einer kleineren slew-rate während der offener Regelkreis Charakterisierung erklärt werden, während bei der strahlbasierten Messung höhere slew-rates zu erwarten sind. Damit ergibt sich eine Verschiebung der Pole und Nullstellen gegenüber der bei der Charakterisierung des Gesamtsystems gefundenen Positionen.

Zur Demonstration des Einflusses der räumlichen Modellfehlanpassung auf das gesamte COFB-Systems und um die Frequenz der Polstellen zu finden, wurde der Strahl angeregt während das Rückkoppelsystem eingeschaltet war. Das geschlossener Regelkreis System zeigt als Antwort eine gedämpft oszillierendes Verhalten aus dem die Anstiegs- bzw. Abfallszeit und die Frequenz gemessenen wurde um damit die Position des dominierenden Pols in der komplexen Ebene bestimmen zu können. Die störende Oszillationsfrequenz lag bei dieser Messung bei $335 \mathrm{~Hz}$ und, für abnehmendes $\rho(\mathbf{M})$, änderte sich das Vorzeichen der Dämpfungskonstante (d.h. der Pol wanderte von rechten auf die linke Seite der komplexen Ebene). Da die Regelparameter kleiner gewählt wurden als die ursprünglichen theoretischen Werte, wurde das geschlossener Regelkreis System erst nahe bei $\rho(\mathbf{M})=1$ instabil. Damit konnte die Funktionalität des COFB-Systems demonstriert und die in dieser Arbeit abgeleiteten Vorhersagen bestätigt werden. 


\section{List of Tables}

2.1 Transverse closed orbit stability requirements for some synchrotron light sources worldwide. . . . . . . . . . . . . . . . . . . . 10

2.2 Sources of perturbations and their typical time and length scales $[26]$. . . . 12

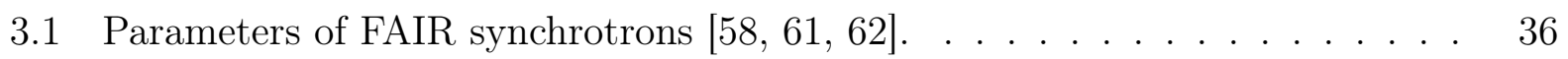

$3.2 \quad$ Parameters for the upgraded SIS18 as the booster ring of SIS100 [16, 62]. . . 37

3.3 Dimensions of the vacuum chambers for dipole and quadrupole magnets in SIS18 |51|. . . . . . . . . . . . . . . . . . . . . . . 40

5.1 Calculated spectral radius in case of symmetric approximations for SIS100

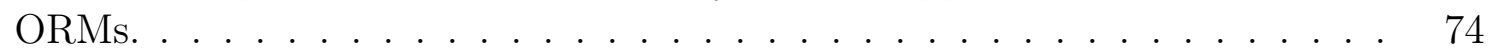

6.1 The symbols of the signals and matrices used in the COFB algorithm. . . . . 99

6.2 Available register width and bit cutting at each node of the corrector-settings calculation. The center value in the third column is retained while left and right number of bits are cut |90|. For meaning of the symbols see Table. 6.1] 102

7.1 Comparison of SIS18 and SIS12 modes of main dipole configuration [93]. . . 106

7.2 Parameters extracted from the system identification for the corrector magnets 4 and 11 along with their power converters. $p$ and $z$ represent the poles and zeros, respectively. . . . . . . . . . . . . . . . 110

7.3 Parameters extracted from the full-system identification for the corrector channels 4 and 8. $p$ and $z$ represent the poles and zeros, respectively. . . . . 117

7.4 PI controller parameter estimation for measured transfer functions of corrector channels 4 and $8 . \ldots \ldots \ldots$. . . . . . . . . . . . . . . . . . . . . . 118

7.5 Information of the step disturbance applied to the SIS18 COFB system in the closed-loop configuration. The last value corresponds to the ramp of orbit correction with measured injection ORM while others are for MAD-X ORM. 131 


\section{List of Figures}

2.1 One section of SIS18 hadron synchrotron showing the RF cavity, two dipole magnets and three quadrupole magnets. . . . . . . . . . . . . . .

2.2 Schematic example of ideal closed orbit (dashed black) and particle trajectories around the closed orbit. . . . . . . . . . . . . . . . . 7

2.3 Schematic example of ideal (dashed black) and perturbed closed orbit in horizontal (blue) and vertical (red) planes. . . . . . . . . . . . . . . . . . 9

2.4 A simple RC circuit. When the switch $\mathrm{S}$ is connected to a, the capacitor is charged by the emf $\xi$ through the resistor $R$. . . . . . . . . . . . . . . 20

2.5 Mass-spring system with damping proportional to the velocity $v=\frac{d x}{d t}$ and

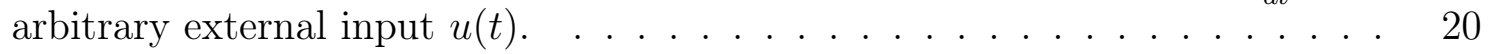

2.6 Transient response of linear systems associated with certain pole location marked as ' $\mathrm{x}$ '. Stable systems are required to have poles located in the left-half plane (LHP), whereas unstable systems have poles in right-half plane of the

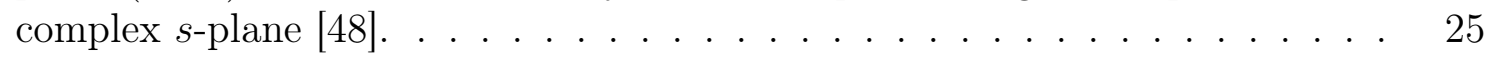

$2.7 \quad$ Example of a typical Bode plot for a second order system $|36|$. . . . . . . . 26

2.8 Schematic diagram of a basic feedback loop. . . . . . . . . . . . . . . . 27

2.9 Left: Scheme of the position measurement using the so-called linear cut BPM and an example of an electrode arrangement for the horizontal plane. Right: Schematic drawing for a linear cut BPM for both planes |54|. . . . . . . . . 30

2.10 Schematic of an internal model controller (IMC) in $s$-domain for a SISO system. 32

2.11 Sampling of a delayed signal. . . . . . . . . . . . . . . . . . . 33

3.1 Layout of the upcoming FAIR facility 60 . SIS18 is the booster ring of SIS100 synchrotron. . . . . . . . . . . . . . . . . . . . . 36

3.2 Measured transverse beam profile in horizontal plane for SIS18 over various times from injection to extraction. . . . . . . . . . . . . . . . 37

3.3 Top: Triplet to doublet quadrupole transition over the ramp of $10 \mathrm{~T} / \mathrm{s}$ in SIS18 (an example of optics change during acceleration cycle). $\mathrm{K} 1_{f 1}, \mathrm{~K} 1_{d 2}$ and $\mathrm{K} 1_{f 3}$ are the normalized strengths of first, second and third singlets quadrupole families of SIS18, respectively. Bottom: The variation of the highest singular values of the ORM in both transverse planes, over the ramp of $10 \mathrm{~T} / \mathrm{s}$. . . . 38 
3.4 Beta function corresponding to the quadrupole strengths at injection and extraction related to the focusing strengths depicted in Fig. 3.3 for SIS18. Horizontal (top) and vertical (bottom). . . . . . . . . . . . . . . . . 39

3.5 Measured tune variation over a typical ramp in SIS18 |53|. . . . . . . . . . . 40

3.6 Mains frequency and its higher harmonics coupled to the closed orbit in SIS18. 41

3.7 Measured beam position over 30 consecutive cycles at two BPM locations in SIS18, for injection and extraction settings. Measurement was made in July

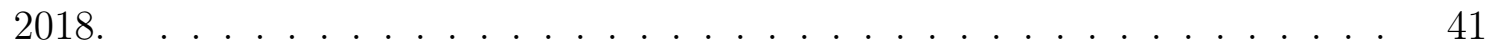

4.1 Comparison of sliding bump and SVD method for the correction of a static orbit correction in SIS18 (simulation in MAD-X). . . . . . . . . . . . . . 44

4.2 SVD modes (columns of $\mathbf{U}$ (blue) and V (orange) matrices). Left: vertical plane. Right: horizontal plane. . . . . . . . . . . . . 46

4.3 Schematic of the lattice of the SIS18 synchrotron. The first section has been magnified on top right and the components are labelled. The horizontal correctors are located at the $1^{\text {st }}$ dipole as extra windings in all sections except in the $4^{\text {th }}$ and $6^{\text {th }}$ sections where they reside on the second dipoles (not labeled in the figure). . . . . . . . . . . . . . . . . . . . 48

4.4 Fourier modes of order $m$. (Real (black dots) and imaginary (red dots) parts of the columns of Fourier matrix F). . . . . . . . . . . . . . . . . 50

4.5 Top: Comparison of singular values of SIS18 ORM with its nearest-circulant approximation and its pure-circulant approximation in the $x$-plane. Bottom: Measured closed orbits in the $x$-plane of SIS18. Red( (**): Perturbed orbit $(\mathrm{RMS}=7.12 \mathrm{~mm})$. Blue(' $\mathrm{x}$ '): Corrected orbit using original ORM (RMS $=2.15$ $\mathrm{mm})$. Black $\left({ }^{\circ} \diamond^{\prime}\right)$ : Corrected orbit using nearest-circulant approximation $(\mathrm{RMS}=2.82 \mathrm{~mm}) . \ldots \ldots \ldots \ldots \ldots$. . . . . . . . . . . . . . . . . . . . . .

4.6 Beta function at BPM locations in the y-plane of SIS100 (simulated in MAD-X) plotted for three cells, with beta beating $\left(\operatorname{red}\left({ }^{(*}\right)\right)$ and without beta beating (blue ('x')). The abscissa is the distance along the synchrotron. . . . . . . . . 58

4.7 Magnitudes of the complex entries of the block diagonal matrix $\mathbf{D}$ defined in Eq. 4.38 calculated for the case of ignoring the beta beating in the $x$-plane of SIS100. . . . . . . . . . . . . . . . . . . . . . . . . . . .

4.8 Magnitudes of the complex entries of the block diagonal matrix $\mathbf{D}$ defined in Eq. 4.38 , calculated for the case of ignoring the beta beating in the $y$-plane of SIS100. . . . . . . . . . . . . . . . . . . . . 59

4.9 Tune modes of DFT matrix $\left(\mathbf{F}_{6}^{*} \otimes \mathbf{F}_{14}^{*}\right)$ (blue, solid) and SVD matrix U (red, dotted) for SIS100 ORM in the x-plane. The horizontal tune is 18.87. . . . . 59 
4.10 Comparison of the SVD singular values with the magnitudes of the rows of matrix D defined in Eq. 4.38 for the SIS100 ORM in x-plane. The abscissa is the index of singular values of the matrix $\mathbf{S}$ as well as the index of rows of matrix D (after sorting in the descending order of their magnitude). . . . . . 60

4.11 Top: Prediction of the closed orbit position at the "missing" BPM locations. Red('*'): Simulated perturbed orbit in MAD-X in the y-plane of SIS18. Black('o'): Sampling of perturbed orbit at BPM locations. Green('*'): Random initial guess for the orbit position at the two "missing" BPM locations. Blue(' $\mathrm{x}$ '): Estimated orbit position at "missing" BPM locations using DFT mode structure of the ORM. Bottom: Simulation of orbit correction for various cases of "missing" BPMs. Red('*) : Simulated perturbed orbit in MAD-X in y-plane of SIS18. Magenta(' $\diamond$ '): Corrected orbit using all BPMs. Green(' $\curlywedge$ '): Corrected orbit using the predicted orbit position for the "missing" BPMs. Black(' $\mathrm{x}$ '): Corrected orbit excluding the rows corresponding to the "missing" BPMs from ORM. Blue('o'): Corrected orbit by taking zero orbit position at the "missing" BPM locations. . . . . . . . . . . . . . . . . 61

4.12 Measured horizontal closed orbits in SIS18 for the various average momentum offsets represented as \% with each orbit. . . . . . . . . . . . . . . . 63

4.13 Coupling of experimentally measured DC shift $\Delta x(\mathrm{~mm})$ in the horizontal closed orbit to the last two columns of the $\mathbf{U}$ matrices $\left(\mathbf{u}_{12}\right.$ and $\left.\mathbf{u}_{11}\right)$ of the original ORM and its nearest-circulant approximation. . . . . . . . . . 63

$5.1 \quad$ Schematic of closed orbit feedback loop in $z$-domain. . . . . . . . . . . . . . 67

5.2 Schematic of the digital closed orbit feedback system in the slow regime. $-\mathbf{r}(z)$ is the error signal, $\boldsymbol{\Theta}(z)$ is the corrector settings, $\mathbf{r}(z)$ is the residual orbit and

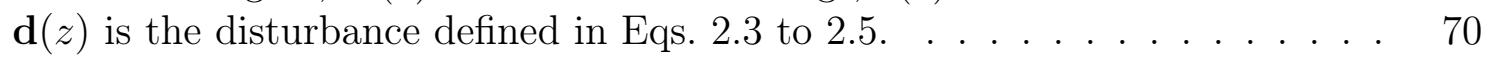

$5.3 \quad$ System interconnection for the application of small gain theorem. . . . . . . 73

5.4 The variation of the highest singular values of the ORMs in both transverse planes, over the ramps of dipole graidents of $5 \mathrm{~T} / \mathrm{s}$ and $10 \mathrm{~T} / \mathrm{s}$. . . . . . . . 76

5.5 The simulated correction ratio $\delta_{1}=\frac{\mathbf{r}_{1, \mathrm{RMS}}}{\mathbf{w}_{0, \mathrm{RMS}}}$ and $\rho(\mathbf{M}(t))$ over a ramp of $10 \mathrm{~T} / \mathrm{s}$ for both planes. . . . . . . . . . . . . . . . . . . 77

5.6 Correction ratio $\delta_{k}=\frac{\mathbf{r}_{k, \mathrm{RMS}}}{\mathbf{r}_{k-1, \mathrm{RMS}}}$ over subsequent iterations in agreement with Gelfand's tormula. . . . . . . . . . . . . . . . . 78

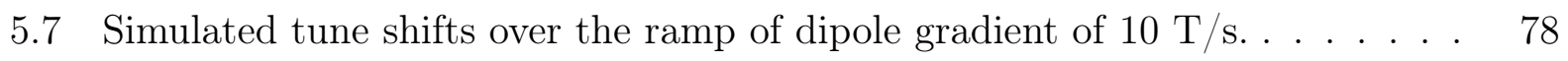

$5.8 \rho(\mathbf{M})$ for both transverse planes over the ramp of dipole gradient of $10 \mathrm{~T} / \mathrm{s}$ for ideal quadrupole strengths as well as filtered for the tune variations. . . . 79

$5.9 \rho\left(\mathbf{M}\left(\Delta Q_{y}\right)\right)$ for image charge based tune shift for three different initial vertical tunes. SGT means small gain theorem. . . . . . . . . . . . . 80 
5.10 Simulated closed orbit RMS over successive time steps of correction in MAD-X for a fixed $\Delta Q=-0.079$ in the slow regime of SIS18 for three initial machine tunes.

$5.11 \rho\left(\mathbf{M}\left(\frac{\Delta \beta}{\beta}\right)\right)$ for beta beating produced by randomly distributed field errors for an initial vertical tune $Q_{y 0}=3.287$. Top: Versus beta beating. Bottom: Versus tune shift. . . . . . . . . . . . . . . . . . . 82

$5.12 \rho\left(\mathbf{M}\left(\frac{\Delta \beta}{\beta}\right)\right)$ for beta beating produced by randomly distributed field errors for an initial vertical tune $Q_{y 0}=3.770$. Top: Versus beta beating. Bottom: Versus

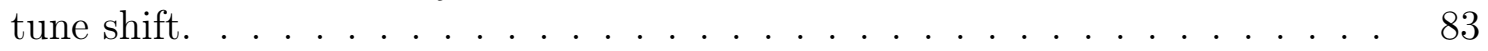

5.13 Measured ratio $\delta_{1}=\frac{\mathbf{r}_{1, \mathrm{RMS}}}{\mathbf{y}_{0, \mathrm{RMS}}}$ in the vertical plane of COSY for the fixed machine tune of $Q_{y 0}=3.62$ while varying the ORM in the feedback system corresponding to a tune range of 3.53 to 4.16 (top). Calculated $\rho(\mathbf{M})$ for vertical plane (bottom). SGT means small gain theorem. For ideal and realistic case please see the text. . . . . . . . . . . . . . . . . . . . 85

5.14 Measured RMS of the closed orbit in $y$-plane of COSY synchrotron for different ORMs used for the corrector settings calculation while keeping the machine settings fixed for a tune of 3.62. . . . . . . . . . . . . . . . . . . . . 86

5.15 Measured RMS of the closed orbit in $x$-plane (top) and in $y$ - plane (bottom) for machine settings corresponding to injection, mid-ramp and extraction energy while using the ORM corresponding to extraction settings for the corrector-settings calculation. . . . . . . . . . . . . . 87

5.16 Sensitivity function of internal model controller (IMC) vs. frequency for various values of $\rho(\mathbf{M})$ between 0 and 1. . . . . . . . . . . . . 90

5.17 Reduction in the cut-off frequency as a function of $\rho(\mathbf{M})$ (lower $x$-axis) and delay (upper $x$-axis). $\ldots \ldots \ldots \ldots . \ldots \ldots 1$

5.18 The closed-loop poles of complementary sensitivity function moving from leftto the right-hand side the the complex plane with the increase of spatial model mismatch characterized by $\rho(\mathbf{M})$. Three open-loop systems are considered having different pure delays. . . . . . . . . . . . . . . . . . . 92

$6.1 \quad$ SIS18 COFB design overview. For the meanings of symbols see Table 6.1$]$. . 98

6.2 Schematic of the PI controller for $j^{\text {th }}$ SVD mode. IAC stands for Integrator accumulator. . . . . . . . . . . . . . . . . . . . . 98

6.3 $\quad$ Frequency response of Libera Hadron and DAC card. The points show the measurement while the lines show the linear fit. . . . . . . . . . . . . . . . 100

6.4 Comparison of corrector-settings calculation between Libera Hadron, simulation with bit cutting and calculation without bit cutting. . . . . . . . . . 103

7.1 Measured Bode plots for all 12 horizontal corrector magnets along with their power converters and ACUs, in SIS18 mode. . . . . . . . . . . . . . . . . . . 108 
7.2 Measured and fitted (in MATLAB) Bode plots for corrector magnets 4 and 11 with their power converters. . . . . . . . . . . . . . . . . . 109

7.3 Pulse response of corrector magnet 4 along with its power converter and ACU for SIS18 (green) and SIS12 modes (blue) of main dipole configuration. The input signal (red) have amplitudes of $0.7 \mathrm{~A}, 6 \mathrm{~A}, 12 \mathrm{~A}, 18 \mathrm{~A}$ and $36 \mathrm{~A}$ reached

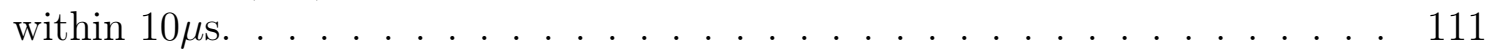

7.4 Pulse response of corrector magnet 11 along with its power converter and ACU for SIS18 (green) and SIS12 modes (blue) of main dipole configuration. The input signal (red) have amplitudes of 0.7A, 6A, 12A, 18A and 36 A reached within $10 \mu \mathrm{s} . \ldots \ldots \ldots \ldots \ldots$. . . . . . . . . . . . . . . . . . . . . . . .

7.5 Bode plots for horizontal corrector 4 and its power converter in SIS18 mode $\begin{array}{lll}\text { extracted from its responses to the pulse inputs of variable heights per } 10 \mu \mathrm{s} . & 113\end{array}$

7.6 Dominant poles and zeros of estimated system models for various heights of

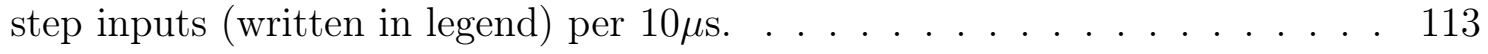

7.7 Schematic of the hardware arrangement for the open-loop system identification. 114

7.8 Measured Bode plots for the full-system for 6 of 9 similar horizontal corrector channels for sinusoidal excitation of $0.7 \mathrm{~A}$ amplitude. . . . . . . . . . . . . . 115

7.9 Measured Bode plots for the full-system for $4^{\text {th }}, 8^{\text {th }}$ and $11^{\text {th }}$ (similar to $10^{\text {th }}$ ) horizontal corrector channels for sinusoidal excitation of 0.7A amplitude. . . 115

7.10 Measured and fitted Bode plot for the full-system for $4^{\text {th }}$ horizontal corrector channel for sinusoidal excitation of 0.7A amplitude. . . . . . . . . . . . 116

7.11 Measured and fitted Bode plot for the full-system for $8^{\text {th }}$ horizontal corrector channel for sinusoidal excitation of 0.7A amplitude. . . . . . . . . . . . . . 116

7.12 Example of closed orbit measured at 3 BPMs over the ramp as result of sinusoidal excitation of $100 \mathrm{~Hz}$ at corrector 1 for the ORM measurement (normalized with beam rigidity over the ramp). . . . . . . . . . . . . . . 119

7.13 Measured singular values over a ramp of dipole field gradient of $1.4 \mathrm{~T} / \mathrm{s}$ at a step of $4 \mathrm{~ms}$ over a time window of $14 \mathrm{~ms}$ for each data point. . . . . . . . . 119

7.14 Measured spatil model mismatch over a ramp of dipole field gradient of 1.4

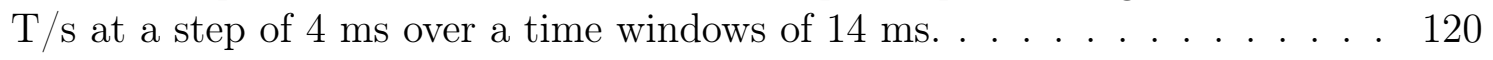

7.15 Measured spatial model mismatch over a ramp of dipole field gradient of 1.0

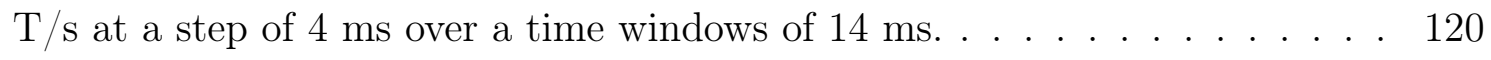

7.16 Orbit correction over the ramp in BPM space for the case of using MAD-X based model ORM corresponding to the injection energy in the controller. Measured beam position on all BPMs in SIS18 for uncorrected orbit (above) and for corrected orbit up to $260 \mathrm{~ms}$ (below). . . . . . . . . . . . . . . . . 122 
7.17 Orbit correction over the ramp in BPM space for the case of using MAD-X based model ORM corresponding to the injection energy in the controller. Measured beam position on all BPMs in SIS18 for uncorrected orbit (above) and for corrected orbit from $40 \mathrm{~ms}$ to $350 \mathrm{~ms}$ (below) using $K_{p}=0.046$ and

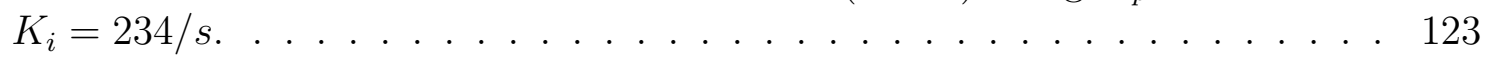

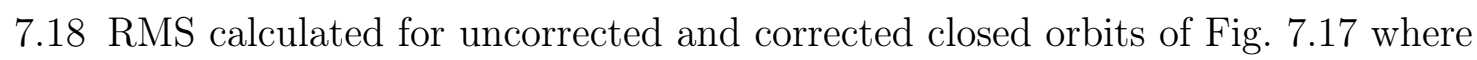
$K_{p}=0.046$ and $K_{i}=234 / s$ for the orbit correction. . . . . . . . . . . . . . . 124

7.19 Golden orbit manipulation during the orbit correction over the ramp. Measured beam position on all BPMs in SIS18 for corrected orbit from $40 \mathrm{~ms}$ to $350 \mathrm{~ms}$ using $K_{p}=0.046$ and $K_{i}=234 / s$. The MAD-X based model ORM corresponding to the injection settings is used in the controller. . . . . . . . . 124

7.20 Orbit correction for SIS18 in frequency domain (average of the DFT of position data from all BPMs) for various controller gains for the case of using MAD-X based model ORM corresponding to the injection settings in the controller. . 125

7.21 Orbit correction over the ramp in BPM space. Measured beam position on all BPMs in SIS18 for uncorrected orbit (above) and for corrected orbit from 40 ms to $540 \mathrm{~ms}$ (below) for the case of using measured ORM corresponding to the injection settings and controller gains of $K_{p}=0.37$ and $K_{i}=2343 /$ s. . . 127

7.22 Average of the DFT of position data from 9 BPMs (excluding BPMs 2, 6 and 12) for COFB OFF (red) and COFB ON (blue) with $K_{p}=0.37$ and $K_{i}=2343 / s$, using measured ORM corresponding to the injection settings in the controller. For explanation of low frequency enhancement, please see text. 128

7.23 Closed orbit excitation for higher controller gains for the case of using MAD-X based model ORM corresponding to the injection settings in the controller. The induced oscillations are in the region of maximum $\rho(\mathbf{M})$. . . . . . . . . 129

7.24 Closed orbit instability (average of the DFT of position data from all BPMs) as a function of higher controller gains for the case of using MAD-X based model ORM corresponding to the injection settings in the controller. . . . . 130

7.25 Schematic diagram of closed-loop system identification. The step input is applied at the output of the controller as a multiplication factor. . . . . . . . 131

7.26 The closed-loop response of the SIS18 COFB system coupled to the one of the least varying SVD modes $\left(4^{t h}\right)$ of MAD-X ORM over the ramp, to the step inputs at various positions on the ramp (i.e. at different magnitudes of spatial model mismatch). . . . . . . . . . . . . . . . . . . . 132

7.27 Decay constant of the beam motion coupled to one of the least varying SVD modes $\left(4^{\text {th }}\right)$ of MAD-X ORM over the ramp, as a response to the step change at various times on the ramp (i.e. at different magnitudes of spatial model mismatch), plotted in Fig. 7.28 . . . . . . . . . . . . . . . . . 133 
7.28 Pole movement as a function of model mismatch over the ramp measured by the fitting of step responses of beam motion coupled to the one of the least varying SVD modes $\left(4^{\text {th }}\right)$ of MAD-X ORM over the ramp, plotted in Fig. 7.26 . 134

7.29 Closed-loop response to the step disturbance at $200 \mathrm{~ms}$ of the ramp for which $\rho(\mathbf{M})=0.58$. Further details in text below. . . . . . . . . . . . . . . 135

A.1 Architecture Overview of the Accelerator Control System. Picture copied as it

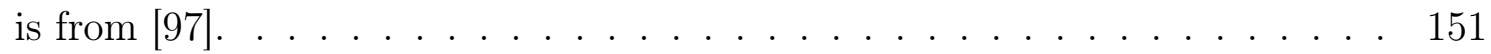

A.2 Libera Hadron hardware layout. For abbreviations please see the text below. 152

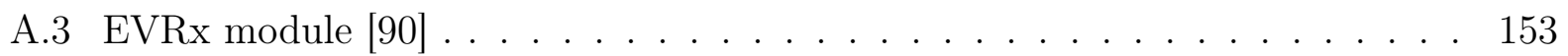

A.4 Local data concentration from HBPM modules to GDX module [90]. . . . . 154

B.1 Front board of Multifunction Module of ACU unit. . . . . . . . . . . . . . . 156

B.2 A screen shot of "Power Config Advanced" software to set the controller parameters in the adaptive control units connected to the horizontal corrector power converters of SIS18. . . . . . . . . . . . . . . . . . . 157

xvii 


\section{List of symbols}

BPM Beam position monitor

COFB Closed orbit feedback

COSY Cooler synchrotron

DFT Discrete Fourier transform

FPGA Field-programmable gate array

IMC Internal model controller

MADX Methodical Accelerator Design

MICADO MInimisation des CArres des Distortions d'Orbite

MIMO Multiple-input-multiple-output

ORM Orbit response matrix

PI Proportional Integral

RMS Root mean square

$\mathbf{R}_{C} \quad$ Circulant ORM

$\mathbf{R}_{B C} \quad$ Block-circulant ORM

SGT Small gain theorem

SISO Single-input-single-output

SIS Schweres Ionensynchrotron (Heavy ion synchrotron)

SVD Singular value decomposition

$\rho(\mathbf{M})$ Highest eigenvalue of matrix $\mathbf{M}$ 


\section{Contents}

Page

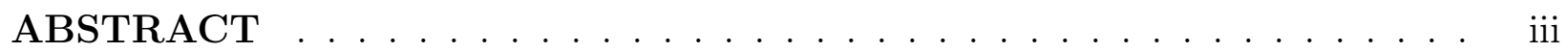

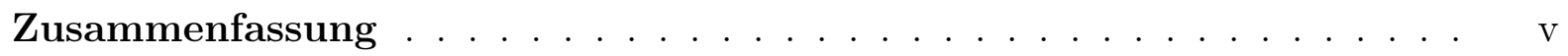

LIST OF TABLES . . . . . . . . . . . . . . . . . . . . . . . . . $\mathrm{x}$

LIST OF FIGURES . . . . . . . . . . . . . . . . . . . . . . . . . . . . . . . xi

LIST OF SYMBOLS $\ldots \ldots \ldots \ldots \ldots$. . . . . . . . . . . . . . . . . . . . . . . .

1 Introduction . . . . . . . . . . . . . . . . . . . 1

2 Closed orbit feedback system in synchrotrons . . . . . . . . . . . . . 4

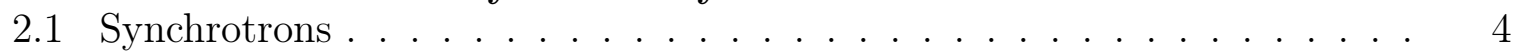

2.2 Closed orbit in a synchrotron . . . . . . . . . . . . . . . . . . . . 6

2.3 Closed orbit distortion $\ldots \ldots \ldots \ldots$. . . . . . . . . . . . . . . . 7

2.4 Disadvantages of closed orbit distortion in synchrotrons . . . . . . . . . . 9

2.4 .1 Synchrotron light sources $\ldots \ldots \ldots \ldots$

2.4 .2 Hadron synchrotrons and particle colliders . . . . . . . . . . . . 11

2.5 Sources of closed orbit distortions $\ldots \ldots \ldots \ldots \ldots \ldots \ldots \ldots$

2.5 .1 Long term perturbations ( $\sim$ hours, days and years, $<<1 \mathrm{~Hz}) \ldots 12$

2.5 .2 Medium term perturbations $(\sim$ few seconds, $1-50 \mathrm{~Hz}) \quad \ldots \ldots . .12$

2.5.3 Short term perturbations (fractions of seconds, several $100 \mathrm{~Hz}$ ) . . 12

2.6 Closed orbit correction $\ldots \ldots \ldots \ldots \ldots \ldots$

2.7 Methods of correction calculation for correctors. . . . . . . . . . . . . . . 13

2.7 .1 Sliding bump method . . . . . . . . . . . . . . . . . . . . . . . 13

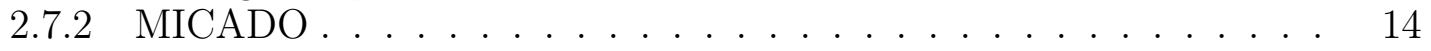

2.7 .3 Harmonic correction . . . . . . . . . . . . . . . . . . . . . . 15

2.7 .4 Singular value decomposition of ORM . . . . . . . . . . . 15

2.8 Closed orbit feedback system $\ldots \ldots \ldots \ldots \ldots \ldots \ldots$

2.8 .1 Fundamentals of control theory . . . . . . . . . . . . . . . . . . 19

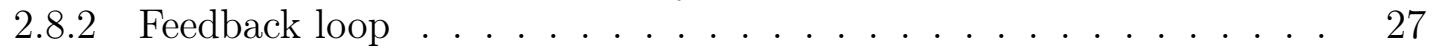

$2.8 .3 \quad$ System model for closed orbit feedback (COFB) system . . . . . . 28

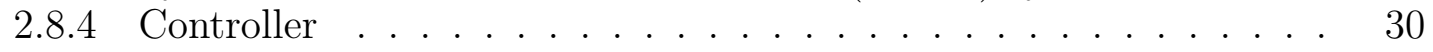

2.8 .5 The z-transform . . . . . . . . . . . . . . . . . 33

3 Problem description: on-ramp fast closed orbit correction in SIS18 . 35

3.1 Motivation of orbit correction at SIS18 $\ldots \ldots \ldots \ldots \ldots \ldots$

3.2 Peculiar behaviors of SIS18 synchrotron in the context of COFB system. 37 
3.3 Demands for the SIS18 COFB system . . . . . . . . . . . . . . . . . . . 42

4 Symmetry exploitation in the orbit response matrix . . . . . . . . . 43

4.1 Orbit correction methods: a brief comparison . . . . . . . . . . . . . . . 43

4.2 Limitations of SVD-based orbit correction . . . . . . . . . . . . . . . . . 45

4.3 Symmetry exploitation in the ORM . . . . . . . . . . . . . . . . . . . 47

4.3 .1 Circulant symmetry of SIS18 vertical ORM . . . . . . . . . . . . . 48

$4.3 .2 \quad$ Decomposition and inversion of the ORM . . . . . . . . . . . . . 50

$4.3 .3 \quad$ Equivalence of SVD and DFT for circulant symmetry . . . . . . . 51

$4.3 .4 \quad$ Broken symmetry and nearest-circulant approximation. . . . . . . 53

4.3.5 $\quad$ Block-circulant symmetry in the ORM . . . . . . . . . . . . . . 55

$4.3 .6 \quad$ SIS100 ORMs and symmetry exploitation . . . . . . . . . . . . . . 57

4.4 Advantages of symmetry exploitation . . . . . . . . . . . . . . . 60

4.4.1 Orbit correction in the case of malfunctioning BPMs . . . . . . . . 60

4.4 .2 Computational complexity _..................... 62

$4.4 .3 \quad$ Momentum mismatch and orbit correction . . . . . . . . . . . . . 62

4.5 Summary and contributions . . . . . . . . . . . . . . . 64

5 Characterization of spatial model mismatach for a COFB system . . 66

5.1 Closed orbit feedback system in generic form . . . . . . . . . . . . . 66

5.2 Spatial model mismatch $\ldots \ldots \ldots$. . . . . . . . . . . . . . . . . . . . . . . . . . . 68

5.3 Spatial model mismatch in slow temporal regime . . . . . . . . . . . . . . 70

5.3.1 Stability criteria for the slow regime of COFB system . . . . . . . 71

5.3 .2 Small gain theorem $\ldots \ldots \ldots \ldots$. . . . . . . . . 72

5.3.3 Comparison between spectral radius condition and small gain theorem 72

5.3.4 Measurement of the spectral radius . . . . . . . . . . . . 73

5.4 Spectral radius for circulant approximations . . . . . . . . . . . . . . . 74

5.5 Spectral radius calculation for spatial model mismatch in SIS18: simulations 75

5.5 .1 On-ramp ORM variation for SIS18. . . . . . . . . . . . . 75

5.5 .2 Intensity dependent tune shift . . . . . . . . . . . . . . . . . . . 79

5.5 .3 Beta beating . . . . . . . . . . . . . . . . . . . 81

5.6 Experimental verification at COSY FZJ . . . . . . . . . . . . . . . 83

5.6 .1 Vertical tune variation . . . . . . . . . . . . . . . . . 84

5.6 .2 On-ramp ORM variation . . . . . . . . . . . . . . . . . 84

5.7 Spatial model mismatch in the fast temporal regime . . . . . . . . . . . . 86

5.7 .1 Temporal bandwidth . . . . . . . . . . . . . . . . 89

5.7 .2 Closed-loop stability . . . . . . . . . . . . . . . . . . . . . . . . . . 90

5.8 Summary and contributions . . . . . . . . . . . . . . . . . . . . . 92

6 Hardware for SIS18 COFB system . . . . . . . . . . . . . . . . . . . . . . 95

6.1 Basic hardware requirements . . . . . . . . . . . . . . . . . . . . . . . . 95

6.2 Hardware for SIS18 COFB system . . . . . . . . . . . . . . . . . . . . . 96

6.2 .1 Prime modules of Libera Hadron . . . . . . . . . . . . . . . . . . . 96

6.2 .2 Functionalities implemented in GDX module . . . . . . . . . . . . 97

6.3 Testing of Libera Hadron during commissioning $\quad$. . . . . . . . . . . . . . . 99

6.3 .1 Delay measurement . . . . . . . . . . . . . . . . . . . . 100

$6.3 .2 \quad$ Fixed-point arithmetic test . . . . . . . . . . . . . . . . . . 101

$6.3 .3 \quad$ Synthetic data generator for system identification . . . . . . . . . 103 
6.4 Summary . . . . . . . . . . . . . . . . . . . . . . . 103

7 SIS18 COFB system in horizontal plane: orbit correction and char-

acterization . . . . . . . . . . . . . . . . . . . 105

7.1 Components of the COFB system . . . . . . . . . . . . . . . 105

7.2 Open-loop system identification . . . . . . . . . . . . . . . . 106

7.2.1 Sub-system identification: Corrector magnets, power converters and adaptive control units . . . . . . . . . . . . . . . . 106

7.2 .2 Full-system identification in open-loop . . . . . . . . . . . . . . . 111

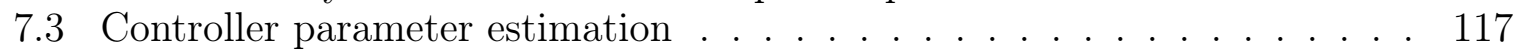

7.4 Orbit response matrix measurement . . . . . . . . . . . . . . . . . . . . . 118

7.5 Closed-loop measurements . . . . . . . . . . . . . . . . . . . . 121

7.5 .1 On-ramp orbit correction . . . . . . . . . . . . . . . . 121

7.5.2 Spatial model mismatch and COFB system stability . . . . . . . . 126

7.5.3 Closed-loop system identification . . . . . . . . . . . . . . . 130

7.6 Summary . . . . . . . . . . . . . . . . . . . . . . . . . . . . 135

8 Summary and outlook f . . . . . . . . . . . . . . . . . . . 137

8.1 Summary . . . . . . . . . . . . . . . . . . . . . . . 137

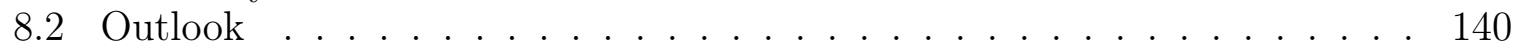

\section{APPENDIX}

$\mathrm{A}$

A.1 GMT of FAIR . . . . . . . . . . . . . . . . . . . . . . . . 150

A.2 Modules of Libera Hadron . . . . . . . . . . . . . . . . . . . . . . 150

A.2.1 FRTN module . . . . . . . . . . . . . . . . . . . . . . . 151

A.2.2 EVRx module . . . . . . . . . . . . . . . . . . . . . . . . . 152

A.2.3 HBPM module . . . . . . . . . . . . . . . . . . . . . . . . 153

A.2.4 GDX module . . . . . . . . . . . . . . . . . . . . . . . . . . . . 153

A.2.5 SER module . . . . . . . . . . . . . . . . . . . . . . . . . . . 154

A.2.6 COFB validity state machine . . . . . . . . . . . . . . . . . . 154

$\mathrm{B} \ldots \ldots \ldots \ldots \ldots \ldots \ldots$

B.1 ACU of corrector power converters . . . . . . . . . . . . . 156 


\section{Chapter One}

\section{Introduction}

This thesis is dedicated to the topic of closed orbit correction in synchrotrons, in general, and the on-ramp orbit correction in hadron synchrotron SIS18 of the FAIR project, in particular. The main goal was to realize a closed orbit feedback (COFB) system for SIS18, efficient enough to achieve the RMS residual below $1 \mathrm{~mm}$ (below 10\% of the typical RMS beam size) throughout the ramp and fast enough to reject disturbances up to the frequencies limited by the accelerator components e.g. corrector magnets and power converters.

COFB system has been an integral part of the synchrotrons and storage rings of light sources as well as of hadron machines for stable beam operations [1-8]. Orbit correction relies on the measurement of transverse beam position at discrete locations in the synchrotron and applying the appropriate kicks to the beam with dedicated dipole magnets located at other discrete locations to counteract the existing field errors in the synchrotron. The linear relation between dipolar kicks and the transverse beam position is described with the help of the orbit response matrix (ORM). A unique feature of SIS18 is the ORM variation over the ramp due to the lattice-settings variation from injection to extraction for every acceleration cycle. The state-of-the-art COFB systems implemented in most other facilities operate for the constant machine settings, e.g. in electron storage rings. The Large Hadron Collider (LHC) is an example of a hadron machine with on-ramp ORM variation but the ramp time of approximately 20 minutes is long enough to update the pre-calculated ORMs for 20 different optics, and the bandwidth of interest lies in the range of several $\mathrm{Hz}[9]$. For light sources, the disturbance rejection is relevant only in the range of DC to $150 \mathrm{~Hz}$ [10].

For SIS18, high-frequency ripples (150 Hz and its higher harmonics) coupled to the beam motion and ramps of very short lengths (200 ms to $1 \mathrm{~s}$, for typical energy gain from 11 $\mathrm{MeV} / \mathrm{u}$ to $1 \mathrm{GeV} / \mathrm{u}$ ) require a larger bandwidth of the feedback system as well as robustness against ORM mismatch. These new challenges not encountered before in any other facility for the COFB system require a thorough study of the existing methods of orbit correction and uncertainty modelling in the ORM in order to answer questions like

- Which orbit correction method is more suitable for the ramping synchrotrons for the computationally efficient update of the ORM and the calculation of the corrector strengths? Existing methods include MICADO[11], harmonic analysis[12], sliding bump method[13] and singular value decomposition of the ORM[14].

- In order to achieve a persistent orbit correction during the ramp of changing lattice settings, what parameter can help decide the rate of the ORM update in the controller? 
- If the COFB system has to run in the fixed-ORM mode during the ramp, is it possible to use fixed controller parameters? If not, how should they scale with the machine ORM variation over the ramp to avoid controller instability?

- Variation of which lattice parameters/functions is crucial, e.g. machine tune or beta function at the location of BPMs and correctors, and how can their influence be characterized?

- What magnitudes of intensity-dependent tune shifts can be tolerated by a COFB system for the required performance and for avoiding instability?

The research conducted to answer the above questions for the realization of a fast and robust SIS18 COFB system has resulted in several new contributions in the field of orbit correction and are presented in this thesis which is arranged as follows:

Chapter 2 comprises basic information required for the understanding of the upcoming chapters and can be divided into two sections. One section is dedicated to the fundamentals of beam dynamics in synchrotrons, including closed orbit distortion, its sources and typical orbit correction methods. The second section describes the basic tools of the control theory required for the design and characterization of a feedback loop.

Chapter 3 is dedicated to the problem statement of the thesis. It sums up the motivation for orbit correction in SIS18 and the challenges associated with its peculiar behavior, sources of the orbit perturbations and comparison of COFB system requirements between SIS18 and other facilities.

In chapter 4, a new discrete Fourier transform (DFT)-based method for the closed orbit correction relying on the symmetry exploitation of the ORM has been introduced. The circulant and block-circulant symmetries in the ORM are discussed, and the supremacy of the DFT-based method over the already existing methods in literature is discussed in terms of information compression, computational benefits, uncertainty modelling and the physical interpretation of the mode space of ORM. A nearest-symmetric approximation is also introduced for the synchrotrons having slight deviation from pure symmetry in the ORM. The advantages of the ORM symmetry exploitation are demonstrated with the help of simulations and experiments for orbit correction in SIS18.

Chapter 5 is the main contribution of the thesis regarding the stability and performance analysis of a COFB system in the presence of ORM mismatch between the controller and the system. A mathematical framework is established in order to model the uncertainty in the ORM and to define a spectral radius condition for the stability of the COFB system. The circulant ORMs' favourable properties are used for a transparent comparison between the already existing condition by small gain theorem and the newly introduced spectral radius condition. Extensive simulations are performed for SIS18 for different sources of ORM mismatch, and the results are verified experimentally at the COFB system of COSY Jülich. The outcome of this chapter has been very helpful for the realization of the SIS18 COFB system.

Chapter 6 introduces the Libera Hadron hardware used for the implementation of the SIS18 COFB system. Different modules of Libera Hadron and their functions are described. The pure delay measurement and the fixed-point arithmetic tests for Libera Hadrons are also 
performed. special features of the hardware, implemented according to the on-ramp orbit correction needs, are also discussed.

Chapter 7 consists of the major experimental part of the thesis and is dedicated to the commissioning and testing of the SIS18 COFB system. It reports about the sub-system and full-system identification, ORM measurement over the ramps and the successful achievement of orbit correction in SIS18 throughout the ramp. The closed-loop characterizations in the light of the conclusions drawn in chapter 5 are also presented.

The summary of the thesis and the outlook for future work are summed up in Chapter 8 . 


\section{Chapter Two}

\section{Closed orbit feedback system in synchrotrons}

In this chapter, the concepts of accelerator physics as well as the control theory are described which form the basis of the work presented in the rest of the chapters. The accelerator related topics include the particle trajectories in the synchrotrons, the closed orbit perturbations, their sources and state-of-the art correction methods. The control theory part describes the representation of the dynamic behavior of the physical systems in the frequency domain and their application to the feedback systems.

\subsection{Synchrotrons}

Synchrotrons are the circular type of charged particle accelerators used for the acceleration as well as storage of high energy particle beams. Synchrotrons can be classified into two broad categories as,

- Synchrotrons where the high energy electron beams act as the source point of the photon beams that are used in the experimental activities. They are knows as synchrotron light sources [15].

- Synchrotrons where the high energy charged beams are themselves used for the experiments. The charged particles cover a wide range from electrons, positrons, protons, antiprotons and heavy ions. They include particle colliders and hadron synchrotrons [16].

The beams are injected into synchrotrons in most cases from the linear accelerators at low energies limited by their physical length and the single-pass trajectory of charged particles through the accelerating structure. In the synchrotrons, the particles are bent around a fixed closed trajectory to have a multiple-pass through the same accelerating structure. The basic acceleration principle is same for any kind of accelerator i.e. the electric field part of the Lorentz force causes the change in the kinetic energy of the charged particles as [17, 18

$$
\begin{aligned}
\Delta E_{k i n} & =q \int(\mathbf{E}+(\mathbf{v} \times \mathbf{B})) d \mathbf{s} \\
& =q \int \mathbf{E} d \mathbf{s}+\underbrace{\left.q \int(\mathbf{v} \times \mathbf{B})\right) \mathbf{v} d t}_{0}
\end{aligned}
$$




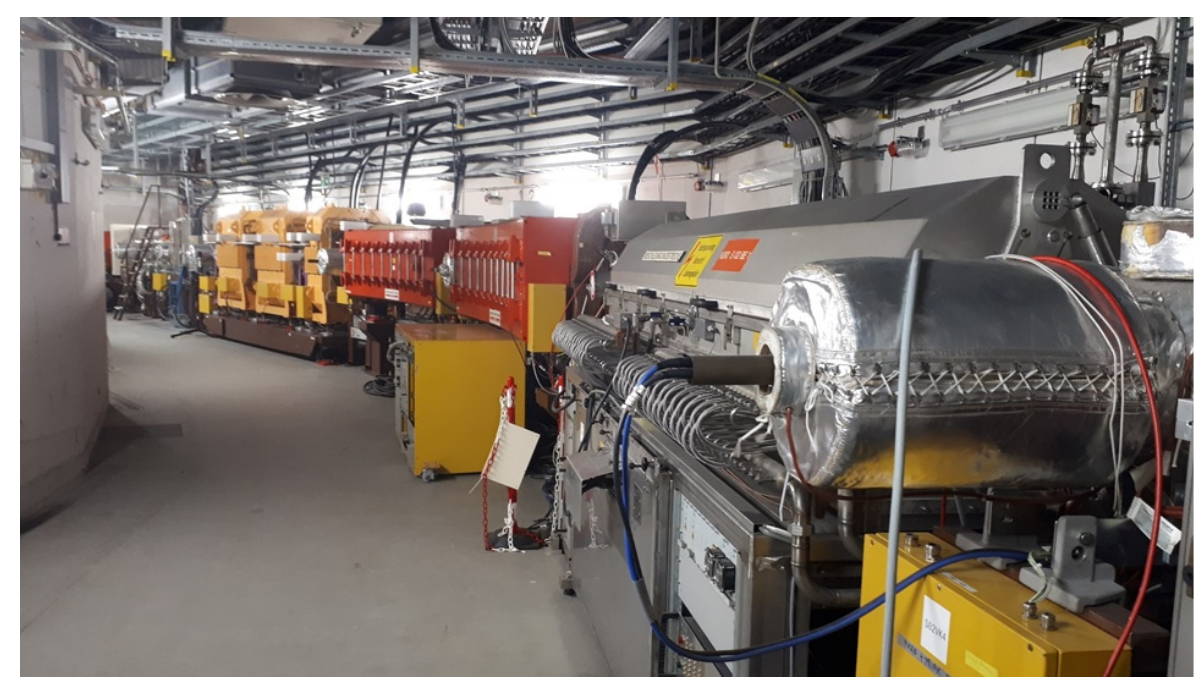

Figure 2.1 One section of SIS18 hadron synchrotron showing the RF cavity, two dipole magnets and three quadrupole magnets.

while the magnetic field is used to bend the particles and is synchronized with the momentum of the particles in order to achieve a fixed radius orbit in a synchrotron. The design condition for a synchrotron is 17,19

$$
B \rho=\frac{p}{q}
$$

where $\rho$ is the gyro-radius of the circular motion in the magnetic field of magnitude $B$ perpendicular to the plane of rotation and $p$ is the momentum of a particle of charge $q$. The term $(B \rho)$ is well knows as the magnetic rigidity and is a measure of the maximum achievable momentum of a charged particle for a given design of the synchrotron which is characterized by the gyro-radius as well as maximum dipole magnetic field. In a synchrotron, the bending magnetic field $B$ is provided by the dipole magnets of finite length distributed uniformly all over the ring while the quadrupole magnets act as the focusing lens for the charged particles with a magnetic field which is a linear function of the particle's transverse position relative to the magnetic center of the quadrupole and is given as [17,

$$
\begin{aligned}
& B_{x}=\frac{\partial B_{x}}{\partial y} y \\
& B_{y}=\frac{\partial B_{y}}{\partial x} x
\end{aligned}
$$

Both dipole and quadrupole magnets along with the straight section form a unit cell which repeats itself to form the full lattice of the synchrotron. Other parts of the synchrotron that lie in the straight sections include the sextupoles, drift spaces and radio frequency (rf) accelerating cavities along with diagnostic and experimental equipment that varies with the type and application of the synchrotron. For example, modern light sources are equipped with Undulators and Wigglers for the emission of high intensity synchrotron radiation [20]. Among diagnostic equipment, one important device is the beam position monitor (BPMs) 
placed in each section in order to measure the transverse position of the beam while small dipole magnets are used to steer the beam at specific locations as well as at the locations of injection and extraction of the beam. Figure 2.1 shows a section of the SIS18 hadron synchrotron of GSI Darmstadt. SIS18 is a strictly periodic lattice with 12 identical cells with a total circumference of $216 \mathrm{~m}$. Each of the 12 periods contains two bending magnets and a quadrupole triplet consisting of two longer singlets and one short singlet at the end, the polarity in $x$-plane is FDF (focus-defocus-focus) [16].

\subsection{Closed orbit in a synchrotron}

The closed path defined by the magnetic centers of the dipole and the quadrupole magnets is referred to as the "golden orbit". A charged particle traveling on this trajectory experiences the "right" centripetal force due to the dipole magnetic field $B$ adjusted with its momentum $p$ according to Eq. 2.2. Such an "ideal" particle faces zero force inside the quadrupoles which provides the focusing field to all off-trajectory "real" particles. The transverse motion $w$ (where $w$ is either $x$ or $y$ ) of such off-trajectory particles inside the linear magnetic structure comprising of dipoles and quadrupole magnets only, as a function of longitudinal coordinate $z$ can be described with the help of a differential equation of the Hill's type given as [17],

$$
\begin{aligned}
w^{\prime \prime}(z)+K_{w}(z) w(z) & =0 \\
w^{\prime}(z) & =\frac{d w}{d z} \\
K_{x}(z) & =\frac{1}{\rho^{2}}+\frac{1}{B \rho} \frac{\partial B_{x}}{\partial y} y \\
K_{y}(z) & =-\frac{1}{B \rho} \frac{\partial B_{y}}{\partial x} x
\end{aligned}
$$

The trajectory of the particles can be written as the solution of Eq. 2.4 as,

$$
w_{\beta}(z)=\sqrt{\epsilon_{w}} \sqrt{\beta_{w}(z)} \cos \left(\phi_{w}(z)-\phi_{0}\right)
$$

where $\epsilon$ and $\phi_{0}$ are the constants of integration. Equation 2.5 shows that the motion of particles in the synchrotron are non-harmonic or -periodic but is a kind of distorted sine-like motion (due to $K$ being a function of $z$ ) with the varying amplitude $\sqrt{\epsilon \beta(z)}$ where $\beta(z)$ is the positive valued function of $z$ and is called beta function having the same periodicity of the circumference $C$ of the ideal orbit as $K(z)$ i.e.

$$
\begin{aligned}
\frac{\partial \sqrt{\beta}}{\partial z} & =K(z) \sqrt{\beta}+\frac{1}{\sqrt{\beta^{3}}} \\
\beta(z) & =\beta(z+C)
\end{aligned}
$$

The phase advance $\phi(z)$ is related to the beta function as

$$
\phi_{w}(z)=\int_{0}^{z} \frac{d z}{\beta_{w}(z)}
$$




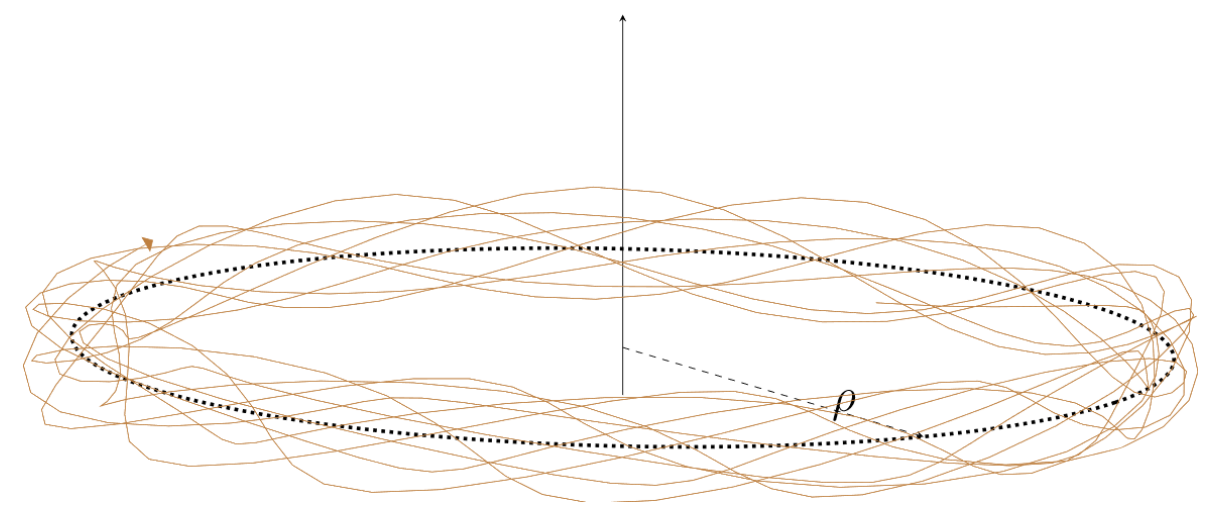

Figure 2.2 Schematic example of ideal closed orbit (dashed black) and particle trajectories around the closed orbit.

The constant of motion $\epsilon$ in Eq. 2.5 is called Emittance and is defined in each transverse plane for the particle motion in the linear field gradient $K(z)$ as,

$$
\epsilon_{w}=\gamma(z) w^{2}(z)+2 \alpha(z) w(z) w^{\prime}(z)+\beta(z) w^{\prime 2}(z)
$$

where $\alpha, \beta$ and $\gamma$ are better called the Courant-Synder functions and defined as,

$$
\begin{aligned}
& \alpha(z)=-\frac{1}{2} \beta^{\prime}(z) \\
& \gamma(z)=\frac{1+\alpha^{2}(z)}{\beta(z)}
\end{aligned}
$$

An example of particle trajectories are plotted in Fig. 2.2 around the ideal orbit for various initial conditions in Eq. 2.5. It is important to mention that the trajectory of individual particles is never closed in one turn and particles make betatron oscillations (defined by Eq. 2.5 around the closed orbit with the total number of oscillations in one turn defined as the tune $(Q)$ given as

$$
Q_{w}=\frac{1}{2 \pi} \int_{z}^{z+C} \frac{d z}{\beta_{w}(z)}
$$

The transverse position of the particles is measured at discrete locations with the help of BPMs and the closed orbit can be extracted as the average of the particle position over many turns for a single particle or as the average of the transverse positions of many particles (forming a beam) over a single turn.

\subsection{Closed orbit distortion}

The ideal closed orbit is valid to exist only in the design magnetic field of a synchrotron given by Eqns. 2.2 and 2.3 and in the absence of any field error. In real synchrotrons, field errors can cause a particle to deviate from the ideal closed orbit even if it originally starts on that orbit. A localized field error of dipolar nature deflects the particle by an angle $\theta$ (mrad) and 
the trajectory for such a distorted closed orbit can be described as the particular solution of the inhomogeneous Hill's equation [17],

$$
w^{\prime \prime}(z)+K_{w}(z) w(z)=\delta\left(z-z_{0}\right) w_{0}^{\prime}
$$

where,

$$
\begin{array}{lrl}
w_{0}^{\prime}=\sin \theta=\frac{\Delta B}{B \rho} l_{\text {mag }} & \text { for the dipole field error } \Delta B \\
w_{0}^{\prime}=\sin \theta=K l_{\text {mag }} \Delta x & \text { for the quadrupole misalignment } \Delta x
\end{array}
$$

with the following boundary conditions required to have the orbit closed at the point of field error $z_{0}$ :

$$
\begin{aligned}
& w_{\text {c.o. }}\left(z_{0}\right)=w_{\text {c.o. }}\left(z_{0}+C\right) \\
& w_{\text {c.o. }}^{\prime}\left(z_{0}\right)=w_{\text {c.o. }}^{\prime}\left(z_{0}+C\right)+w_{0}^{\prime}
\end{aligned}
$$

The particular solution of Eq. 2.11 can be written for small angles $(\sin \theta \approx \theta)$,

$$
w_{\text {c.o. }}(z)=\theta \frac{\sqrt{\beta_{w}\left(z_{0}\right) \beta_{w}(z)}}{2 \sin \left(\pi Q_{w}\right)} \cos \left(\pi Q_{w}-\left|\phi_{w}(z)-\phi_{w}\left(z_{0}\right)\right|\right)
$$

So far we have considered the trajectories for the charged particles with nominal momentum $p$ of Eq. 2.2 both for ideal and distorted closed orbits and the betatron oscillations around them. For a momentum mismatch $\frac{\Delta p}{p}$, the ideal closed orbit is also deviated transversely in the bending magnets and hence primarily in $x$-plane from the ideal orbit as,

$$
x_{D}(z)=D(z) \frac{\Delta p}{p}
$$

where the periodic function $D(z)$ is called the momentum dispersion function and is the solution of Hill's equation in case of $\frac{\Delta p}{p}=1$ given as 21 ,

$$
D^{\prime \prime}(z)+K_{w}(z) D(z)=\frac{1}{\rho(z)}
$$

The final trajectories of charged particles in a synchrotron can be described as the superposition of the perturbed closed orbit and the betatron oscillations around that as,

$$
\begin{aligned}
& x(z)=x_{\beta}(z)+x_{c . o .}+D(z) \frac{\Delta p}{p} \\
& y(z)=y_{\beta}(z)+y_{\text {c.o. }}
\end{aligned}
$$

In the presence of rf system, the revolution time is forced to stay constant in order to maintain synchronism of beam motion with the rf phase. For the perturbed orbit, the beam adopts to the constant revolution time by the slight variation of its energy, which in turn creates a mismatch of beam energy with the dipole fields. As a result, the closed orbit gets an extra contribution proportional to the dispersion in the ring and the field error $\theta$. For the synchrotrons that operate near the transition energy $\left(\gamma_{t}\right)$ or cross it during the ramp, Eq. 2.14 is modified for the horizontal plane in order to keep the orbit length constant as follows [22],

$$
x_{c . o .}\left(z-z_{0}\right)=\theta \frac{\sqrt{\beta_{x}\left(z_{0}\right) \beta_{x}(z)}}{2 \sin \left(\pi Q_{x}\right)} \cos \left(\pi Q_{x}-\left|\phi_{x}(z)-\phi_{x}\left(z_{0}\right)\right|\right)-\theta \frac{D(z) D\left(z_{0}\right)}{\left(\frac{1}{\gamma^{2}}-\frac{1}{\gamma_{t}^{2}}\right) C}
$$




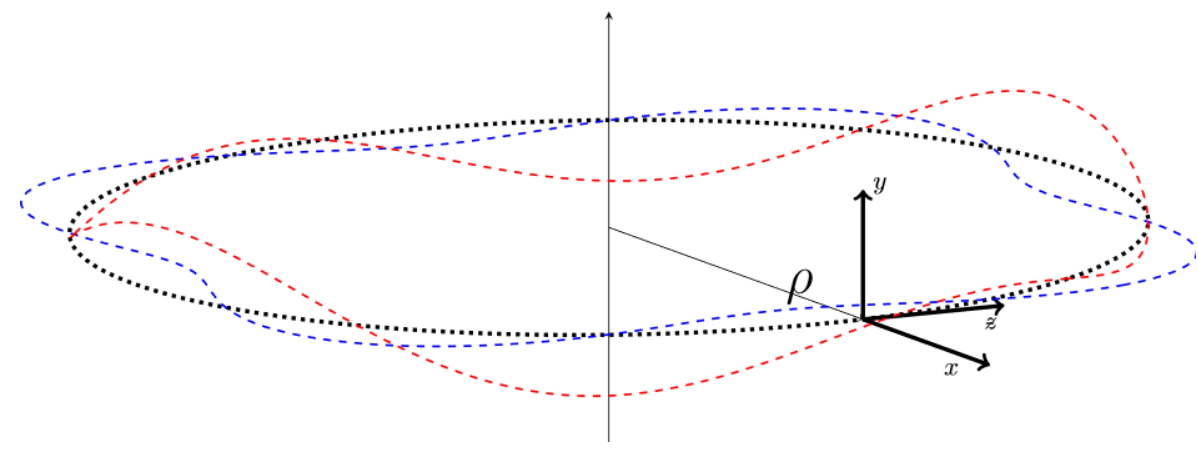

Figure 2.3 Schematic example of ideal (dashed black) and perturbed closed orbit in horizontal (blue) and vertical (red) planes.

\subsection{Disadvantages of closed orbit distortion in synchrotrons}

Closed orbit distortion is an unwanted feature in any kind of synchrotron both in electron and hadron machines as can be (even naively) deduced from the reason of such a distortion i.e. field "errors" (Eq. 2.11). This means that an ideal synchrotron without field errors has no closed orbit distortion and in real synchrotrons the distortion should be as minimal as possible. The justification for the requirement of minimum orbit distortion is very meaningful in terms of accelerator operation and the achievable beam quality in a synchrotron. For example, in real synchrotrons, the larger transverse offset of closed orbit reduces the available aperture for betatron oscillations resulting into the possibility of loss of particles and causes the individual particle trajectories to lie near the poles of the quadrupole magnets. The non-linear field gradient near the poles of the quadrupole magnets and the sextupole magnets can deteriorate the emittance of the particle beams which is a constant of motion only for the linear field gradient. Specific examples of the adverse effects of closed orbit distortion on the accelerator operation for different kinds of synchrotrons are described in the following subsections.

\subsubsection{Synchrotron light sources}

Light sources are the dedicated synchrotrons for the storage of electron beams of fixed energy where the bending electron beam (either in dipole magnets or insertion devices) act as the source of synchrotron radiation which is channeled through optical beamlines to the experimental halls for research purposes. The number of light source synchrotron accelerators has increased in last thirty years remarkably. There are currently more than 50 light sources all around the world fulfilling the experimental needs of the researchers from diverse fields of science and engineering. The general information about these synchrotrons has been gathered on a single web-page www.lightsources.org. These light sources have gone through technological evolution from $0^{\text {th }}$ generation to $4^{\text {rd }}$ generation 23 having nominal beam emittance 5-20 nm-rad and x-ray brightness of the order of $10^{20}$ photons $/ \mathrm{s} / \mathrm{mm}^{2} / \mathrm{mrad}^{2}$. The largest of these synchrotrons include ESRF France, APS USA and SPRING-8 Japan, while recently the synchrotrons based upon the idea of $4^{\text {th }}$ generation light sources to touch the 
Table 2.1 Transverse closed orbit stability requirements for some synchrotron light sources worldwide.

\begin{tabular}{|c|c|c|c|c|}
\hline$\overline{\text { Facility }}$ & $\sigma_{\mathbf{x}}(\mu m)$ & $\overline{\sigma_{y}(\mu m)}$ & $\begin{array}{l}\text { Vertical stability } \\
\text { requirement }\end{array}$ & Reference \\
\hline NSLS II & 32 & 3.2 & $0.3 \mu m$ & 4 \\
\hline PETRA III & 40 & 5 & $0.5 \mu \mathrm{m}$ & $|\overline{6}, \overline{24}|$ \\
\hline DLS (SR) & 123 & 6.4 & $0.6 \mu m$ & $|3|$ \\
\hline ALBA & 53 & 23 & $0.2 \mu m$ & 25 \\
\hline SPEAR3 & 350 & 21 & $<1 \mu m$ & 26 \\
\hline ESRF & 380 & 14 & $1.4 \mu \mathrm{m}$ & 5 \\
\hline BESSY II & 250 & 25 & $2.5 \mu \mathrm{m}$ & {$[\overline{2} \overline{7} \mid$} \\
\hline MAX IV & 22 & 11.5 & $<1 \mu m$ & 28 \\
\hline
\end{tabular}

fundamental limits of x-ray brightness are being realized at ESRF France, MAX IV Swedan, Diamond II UK and SIRIUS Brazil. The concept of beam stability in light sources is primarily defined by the photon beam parameters which are function of electron beam parameters and beam line optical components, measurement devices and experimental samples. For example, the photon beam position error $\Delta y_{p h}$ at a distance $L$ from the source point (without a slit) depends upon the electron beam position error $\Delta y_{e}$ and in case of no focusing is given as [29],

$$
\Delta y_{p h}(L)=\Delta y_{e}+L \Delta y_{e}^{\prime}
$$

where $\Delta y_{e}^{\prime}$ is the electron beam divergence at the source point of photon beam. While in case of slit as the secondary source point of the photon beam, electron beam movement will result into the direct decrease in the intensity of the photon beam. Similarly, the brightness of photon beam directly depends upon the size and divergence of electron beam. The relationship between the beam emittance and brightness is given as [30],

$$
B_{p h} \propto B_{e}=\frac{I_{e}}{8 \pi^{2} \epsilon_{x} \epsilon_{y}}
$$

where $B_{p h}$ and $B_{e}$ are the x-ray and electron beam brightnesses respectively and $I_{e}$ is the electron beam current and $\epsilon_{x}, \epsilon_{y}$ are the emittance in both transverse directions mentioned in Eq. 2.5.

The horizontal beam sizes in the existing synchrotron light sources remains one or two orders of magnitude larger than vertical beam sizes and the general accepted stability criteria for the RMS variation of beam position in synchrotrons is up to $10 \%$ of the RMS beam size, requires sub- $\mu \mathrm{m}$ stabilization of the closed orbit in vertical plane. The required stability criteria for some of the light sources is collected in Table 2.1.

As mentioned before, in synchrotron light sources, the major area of focus is the electron beam stability at the source point of the synchrotron light i.e. in the storage ring. Any emittance growth/distortion due to the non-linear magnetic field gradients in the booster ring can be compensated in the storage ring due to the radiation damping of the vertical 
emittance given as [31],

$$
d \epsilon_{y}=-\epsilon_{y} \frac{d p}{p}
$$

\subsubsection{Hadron synchrotrons and particle colliders}

Synchrotrons where the high energy beams are directly used for the experimental activities include the hadron synchrotrons and particle colliders. In particle colliders, two beams of same or different kind of particles are head-on collided for the production of elementary particles. Relativistic Heavy Ion collider (RHIC) [32] of BNL US, Hadron-Electron Ring Accelerator (HERA) [33] of DESY Germany and Large Hadron Collider (LHC) [34] of CERN Switzerland are among the most famous synchrotron-based hadron colliders in the world. The transverse beam position stability at the interaction point is an important requirement for the collision of two beams while the beam quality deterioration (e.g. brightness as in Eq. 2.20 due to the closed orbit distortion in the non-linear magnetic fields of quadrupole and sextupole magnets decreases the interaction cross-section at the interaction point.

Hadron synchrotrons are the major attraction for nuclear physics community to get relativistic heavy ions. Famous facilities worldwide include Rapid Cycling Synchrotron(RCS) and Main Ring Synchrotron (MR) of J-PARC Japan [35] and the Heavy Ion synchrotron (SIS18) of GSI [16], Germany. A major motivation to keep the closed orbit distortions minimum in hadron synchrotrons come from the fact that heavy ions do not have synchrotron damping even at relativistic energies and hence any beam quality deterioration caused during the injection process and early acceleration remains preserved. Moreover, the ion sources are not as efficient as the electron sources in terms of beam current and intensity. Significant closed orbit distortions can cause beam losses by limiting the aperture for the betatron oscillations. The particle loss can also ruin the vacuum pressure especially in case of heavy ions. Therefore, the closed orbit care is getting more important with the increasing demand of high intensity beams in hadron synchrotrons .

\subsection{Sources of closed orbit distortions}

Perturbations that effect the position of beam center-of-mass in a synchrotron can be divided into three categories in terms of their time period or vibration frequencies; long term, medium term and short term perturbations. The time scale of perturbations depends upon the nature of source producing them. It is worth mentioning here that for each accelerator, the type of perturbations important to be removed depends upon the type and parameters of accelerator itself. The time scale classification is a general classification but the terms long, medium and short are more relative and depend upon the time scale of some specific measurement or phenomenon under consideration, for example, the bunch revolution period considered as

a reference. A list of general sources of perturbations, their time periods and length scales have been shown in Table. 2.2. 
Table 2.2 Sources of perturbations and their typical time and length scales $\mid 26$.

\begin{tabular}{lll}
\hline \hline Source & Time scale & Length Scale \\
\hline Ground settlement & weeks to months & 10 to $100 \mu \mathrm{m} /$ year \\
\hline Earth Tides & hours & $\sim 30 \mu \mathrm{m} / \mathrm{km}$ \\
\hline Air/Water Temperatures & minute to days & $\sim 10 \mu \mathrm{m} /{ }^{\circ} \mathrm{C}$ \\
\hline Beam-induced heating & minutes to hours & $10 \mu \mathrm{m}$ \\
\hline Insertion device parameter changes & $10 \mathrm{~ms}$ to $10 \mathrm{~s}$ & 10 Gauss-cm \\
\hline Stray Fields & 0.1 seconds to hours & variable \\
\hline Magnet power supply noise & $10 \mu$ s to $10 \mathrm{~ms}$ & design dependent \\
\hline Ground Vibrations & $10 \mathrm{~ms}$ to $1 \mathrm{~second}$ & $\sim 10 \mathrm{~nm}$ \\
\hline Magnet vibration (from resonance) & $10 \mathrm{~ms}$ to $1 \mathrm{~second}$ & $\sim 100 \mathrm{~nm}$ \\
\hline RF system & $\mu \mathrm{s}$ to minutes & $\sim 10 \mu \mathrm{m}$ \\
\hline Impedances/Wakefiels & $\mathrm{ns}$ to $\mu \mathrm{s}$ & $\sim 10 \mu \mathrm{m}$ \\
\hline
\end{tabular}

\subsubsection{Long term perturbations ( hours, days and years, $<<1 \mathrm{~Hz}$ )}

These perturbations have time periods of one week or above up to years and correspond to very small frequencies that's why usually described only in terms of time period. Ground settlements and seasonal and environmental changes e.g. temperature changes, rain fall, tides etc. come in this category. These perturbations result in alignment changes of accelerator components e.g. girders and magnets. Orbit drifts caused by such perturbations can reach (for example, for LHC) between 200-300 $\mu \mathrm{m}$ within 10 hours [36]. Such perturbations become significant for storage rings where the beam is stored for long times (for hours). For example, even for smaller machines such as the Swiss Light Source (SLS, $288 \mathrm{~m}$ ) the RF frequency needs to be adjusted to compensate for variations in the ring circumference.

\subsubsection{Medium term perturbations ( few seconds, 1-50 Hz)}

These perturbations mainly include ground vibrations induced by human activities in experimental hall e.g. vibration of mechanical devices and external activities e.g. road traffic noise and range from a fraction of a $\mathrm{Hz}$ to 50 or $100 \mathrm{~Hz}$ maximum. Sources of this category also include movement of the vacuum chambers or quadrupole magnets due to water cooling system etc.

\subsubsection{Short term perturbations (fractions of seconds, several 100 $\mathrm{Hz}$ )}

Short term perturbations usually have sources from the 50 or $60 \mathrm{~Hz}$ mains frequencies and their harmonics (higher frequencies). The major sources include power supply noise, electrical stray fields, booster operation etc. In fast booster rings, the thickness of the vacuum chambers is kept thin enough to avoid eddy currents during the ramping which also allows the power supply high frequency noise to couple to the beam motion. 


\subsection{Closed orbit correction}

Closed orbit correction has become an integral part of almost every synchrotron operation $1-$ 6] and is the process of steering the closed orbit down to the golden orbit against the existing magnetic field errors of dipolar nature, by introducing the artificial field errors (corrections). The perturbed closed orbit is measured with the help of BPMs while dedicated dipole magnets (correctors) are deployed for the application of corrections. BPMs are the transverse beam position readout devices. Corrector magnets can be used to apply local bumps to the orbit in certain positions around the synchrotron or for the orbit correction throughout the ring called local and global orbit correction, respectively. In this thesis, we shall focus on the global orbit correction. The global effect of a single dipolar kick (for small perturbations) $\theta_{c}$ located at a longitudinal location $z_{1}$ is described by the linear solution of Hill's equation given in Eq. 2.14. In case of $M$ BPMs and $N$ correctors, the response of closed orbit at each $\mathrm{BPM}$ to each corrector is given by the elements of $M \times N$ linear matrix called orbit response matrix (ORM) represented by $\mathbf{R}$ such that,

$$
\mathbf{w}=\mathbf{R} \Theta_{w}
$$

where $\Theta$ is the corrector settings vector and $\mathbf{w}$ is the beam position vector at the BPM locations in either transverse plane and the ORM elements are given as,

$$
R_{m n}=\frac{\sqrt{\beta_{m} \beta_{n}}}{2 \sin (\pi Q)} \cos \left(Q \pi-\left|\mu_{m}-\mu_{n}\right|\right)
$$

where $m$ and $n$ represent the BPM and corrector index, respectively. The dispersion term on the right hand side of Eq. 2.18 is ignored in the definition of the ORM in Eq. 2.23 which is only relevant at the transition-crossing during acceleration process. The main concept of global orbit correction is to calculate the corrector-strengths that can counteract the existing dipolar field errors such that the orbit distortion measured with the BPMs is minimized.

\subsection{Methods of correction calculation for correctors}

Historically, four distinct methods have served the global orbit correction which include sliding bump method [13], MICADO [11], harmonic correction [12] and singular value decomposition (SVD) [14. A variant of the SVD type correction referred to as eigenvalue decomposition has also been reported [37, 38. These methods are revised here briefly, before a new method is introduced in the upcoming chapters.

\subsubsection{Sliding bump method}

The sliding bump method is based upon creating a local bump in the closed orbit using any three correctors such that the beam position at a specific BPM location can be made equal to the golden orbit. Once such a bump is created, the set of correctors "slides" to the next three correctors to create a local bump at the next BPM location. The condition on the bump 
angles for three correctors $\left(\theta_{1}, \theta_{2}\right.$ and $\left.\theta_{3}\right)$ is that the resultant closed orbit bump leaves the orbit outside of the corrector region 1-3 unchanged. The bump angle condition is given as,

$$
\begin{aligned}
& \theta_{2}=-\theta_{1} \sqrt{\frac{\beta_{1}}{\beta_{2}}} \frac{\sin \phi_{31}}{\sin \phi_{32}} \\
& \theta_{3}=-\theta_{1} \sqrt{\frac{\beta_{1}}{\beta_{3}}} \frac{\sin \phi_{21}}{\sin \phi_{32}}
\end{aligned}
$$

where $\theta_{1}$ is an arbitrary angle. A local bump does not change the closed boundary condition and no betatron oscillations propagate outside the bump.

The three-corrector-local-bump method is used as following: the uncorrected closed orbit shift $w_{0}$ at one BPM located between correctors 1 and 3 is measured. By varying $\theta_{1}$, the rate of change of closed orbit with the corrector angle $\frac{\partial w}{\partial \theta_{1}}$ is also measured. Within linear region of magnetic lattice,

$$
w=w_{0}+\frac{\partial w}{\partial \theta_{1}} \theta_{1}
$$

where $w$ is the resultant closed orbit position at the location of BPM. By requiring $w=0$, $\theta_{1}$ is calculated to be equal to $\left(w_{0} / \frac{\partial w}{\partial \theta_{1}}\right)$ and then $\theta_{2}$ and $\theta_{3}$ are calculated using Eq. 2.24 in order to close the bump at the location of corrector 1 and corrector3. By repeating this procedure to all correctors in groups of three, the closed orbit is consequently corrected at all BPM positions, in each plane. Anyhow, this method has a fundamental limitation since three correctors are used for the orbit correction at one BPM, the degree of freedom for the correctors is always less than the total number of correctors by 2 i.e. effective number of correctors is $N-2$.

Sliding bump scheme of orbit correction was historically used at SIS18 synchrotron of GSI [39].

\subsubsection{MICADO}

MICADO, also referred to as orthogonal matching pursuit in the signal processing literature $[15,16]$, was devised to find the most effective correctors to minimize the orbit distortion. The name MICADO comes from an algorithm named in French as "MInimisation des CArres des Distortions d'Orbite" meaning Minimization of Orbit Distortions Squares. This method is discussed for the completion of the topic and currently almost no synchrotron facility has reported to use it for the orbit correction, to the best knowledge of the author. The major utility of this method was at the time when orbit distortions were also removed by changing the physical alignment of the quadrupoles and it was very fruitful to locate the regions of maximum magnet defects.

Following strategy is followed in MICADO: At the first stage, each magnet is tested singly and only the corrector correction which minimizes the residual orbit is retained. In the next iteration, the second most effective corrector is identified and the process is repeated unless the peak-to-peak amplitude of the residual orbit vector is smaller than a fixed value given in advance. 


\subsubsection{Harmonic correction}

Harmonic correction was the first method to discuss the notion of mode-based correction by means of a decomposition of the perturbed orbit into Fourier harmonics which can be corrected individually. The technique is primarily based upon the work of L.H.YU and co-authers at BNL US [12], where they introduced the Courant-Snyder variables in Eq. 2.11 as,

$$
\begin{aligned}
\psi & =\frac{\phi}{Q} \\
\eta & =\frac{w}{\sqrt{\beta}} \\
\frac{\partial^{2} \eta}{\partial \phi^{2}}+Q^{2} \eta & =Q \sqrt{\beta_{0}} \theta \delta\left(\psi-\psi_{0}\right)
\end{aligned}
$$

The periodic solution of Eq. 2.26 is given as,

$$
\eta=t \cos \left(Q\left(\pi-\left|\psi-\psi_{0}\right|\right)\right)
$$

where $t=\frac{\sqrt{\beta_{0} \theta}}{2 \sin (\pi Q)}$. Fourier analyzing Eq. 2.27 has been worked out as,

$$
\eta=t \sum_{n=0}^{\infty} c_{n} \cos \left(n\left(\psi-\psi_{0}\right)\right)
$$

where,

$$
\begin{aligned}
& c_{0}=\frac{1}{2 \pi} \int_{-\pi}^{\pi} \cos (Q \psi) d \psi=\frac{1}{Q} \frac{\sin (\pi Q)}{\pi} \\
& c_{n}=\frac{-1^{n}}{\pi} \int_{-\pi}^{\pi} \cos (n \psi) \cos (Q \psi) d \psi=\frac{2 Q}{Q^{2}-n^{2}} \frac{\sin (\pi Q)}{\pi}
\end{aligned}
$$

where $n$ is the harmonic number of Fourier components. Harmonic analysis helps to predict the dominant modes in the perturbed orbit of a synchrotron e.g. the mode closest to the tune value $Q$ always has the ultimate dominance as predicted by Eq. 2.29. The calculation of corrector-strengths $t_{i, n}$ (in terms of Courant-Snyder variables, where $i$ is the corrector index) for the cancellation of selected modes $c_{n}$ in a given perturbed orbit $\eta$ is carried out by the solution of Eq. 2.28 which depends upon the phase relation of BPMs and correctors in the synchrotron. The corrector-strengths are also proportional to the inverse of the Fourier coefficient 12, 40].

\subsubsection{Singular value decomposition of ORM}

Singular value decomposition (SVD) [41, 42] is the most commonly used method for the correction calculation for the correctors and was the first method to rely on the orbit response matrix (ORM) of Eq. 2.23. SVD was first co-introduced by the Volker Ziemann [43] and Y. Chung [14] in 1993 in the closed orbit correction community and since then has become the de-facto method for the orbit correction at synchrotrons.

Before describing the SVD-based correction calculation for the correctors, let us first define the necessary matrix properties. 


\section{Orthogonality of a matrix}

A square matrix $\mathbf{Q} \in \mathbb{R}^{m \times m}$ is said to be orthogonal if $\mathbf{Q}^{T} \mathbf{Q}=\mathbf{I}$ i.e. its inverse is equal to its transpose. And if $\mathbf{Q}=\left[q_{1}|\ldots| q_{m}\right]$ is orthogonal then the columns $\mathbf{q}_{i}$ form the orthonormal basis of $\mathbb{R}^{m}$ where symbol | seperates the columns.

\section{Orthonormality}

For $\mathbb{R}^{m}$ being an inner-product space. A set of vectors $\left\{\mathbf{q}_{i}\right\}$ is called orthonormal if and only if, for all $q_{i}$ and $q_{j}$,

$$
<q_{i}, q_{j}>=\delta_{i j}
$$

where $\delta_{i j}$ is the Kronecker delta and $<,>$ is the inner-product over $\mathbb{R}^{m}$.

\section{Singular value decomposition}

For any real-valued $m \times n$ matrix $\mathbf{R}$, there exist orthogonal matrices,

$$
\begin{aligned}
\mathbf{U} & =\left[u_{1}|\ldots| u_{m}\right] \in \mathbb{R}^{m \times m} \\
\mathbf{V} & =\left[v_{1}|\ldots| v_{n}\right] \in \mathbb{R}^{n \times n}
\end{aligned}
$$

such that

$$
\mathbf{S}=\mathbf{U}^{\mathrm{T}} \mathbf{R V}=\operatorname{diag}\left(\sigma_{1}, \ldots, \sigma_{p}\right) \in \mathbb{R}^{m \times n}, p=\min \{m, n\}
$$

and

$$
\mathbf{R}=\mathbf{U S V}^{\mathrm{T}}
$$

where $\sigma_{1} \geq \sigma_{2} \geq \ldots \geq \sigma_{p} \geq 0$.

$\sigma_{i}$ are called the singular values of matrix $\mathbf{R}$ and the columns $\mathbf{u}_{i}$ and $\mathbf{v}_{i}$ are called left and right singular vectors of $\mathbf{R}$ and are the eigen-vectors of matrices $\mathbf{R R}^{\mathrm{T}}$ and $\mathbf{R}^{\mathrm{T}} \mathbf{R}$, respectively as,

$$
\begin{aligned}
\mathbf{R R}^{\mathrm{T}} \mathbf{u}_{i} & =\sigma_{i}^{2} \mathbf{u}_{i} \\
\mathbf{R}^{\mathrm{T}} \mathbf{R}_{i} & =\sigma_{i}^{2} \mathbf{v}_{i}
\end{aligned}
$$

Singular values are the positive square roots of the eigenvalues of both $\mathbf{R} \mathbf{R}^{\mathrm{T}}$ and $\mathbf{R}^{\mathrm{T}} \mathbf{R}$, by definition. A relation between left and right singular vectors of $\mathbf{R}$ can be defined as a direct consequence of Eq. 2.31 as,

$$
\begin{aligned}
\mathbf{R}^{\mathrm{T}} \mathbf{u}_{i} & =\sigma_{i} \mathbf{v}_{i} \\
\mathbf{R v}_{i} & =\sigma_{i} \mathbf{u}_{i}
\end{aligned}
$$

The orthonormal vectors $\mathbf{u}_{i}$ and $\mathbf{v}_{i}$ are also referred to as the SVD modes which are arranged in the matrices $\mathbf{U}$ and $\mathbf{V}$ as the descending order of their corresponding singular values. In this way, singular values are the measure of the coupling between right and left SVD modes for the system represented by the matrix $\mathbf{R}$. 


\section{Condition number of a matrix}

The condition number $\kappa(\mathbf{R})$ is defined as the ratio of largest to smallest singular value of the matrix $\mathbf{R}$ i.e.

$$
\kappa(\mathbf{R})=\frac{\sigma_{\max }}{\sigma_{\min }}
$$

\section{Pseudo-inversion of a matrix}

The inverse of a matrix is one of the fundamental benefits of SVD and can be defined as,

$$
\mathbf{R}^{-1}=\left(\mathbf{U S V}^{\mathrm{T}}\right)^{-1}=\left(\mathbf{V}^{\mathrm{T}}\right)^{-1} \mathbf{S}^{-1} \mathbf{U}^{-1}=\mathbf{V S}^{-1} \mathbf{U}^{\mathrm{T}}
$$

where e.g. $\mathbf{U}^{-1}=\mathbf{U}^{\mathrm{T}}$ is by definition of the orthogonality of the matrix. For the diagonal matrix $\mathbf{S}$, the inverse is defined as

$$
\mathbf{S}^{-1}=\operatorname{diag}\left\{\frac{1}{\sigma_{1}} \ldots, \frac{1}{\sigma_{p}}\right\}, p=\min \{m, n\}
$$

If any of the singular value $\sigma_{i}=0$, the inverse $\mathbf{S}^{-1}$ does not exist and the matrix is called a singular matrix for which the condition number is $\infty$. A matrix is called ill-conditioned if its condition number is too large than one.

SVD provides the liberty to calculate an approximate inverse even for the singular matrices by replacing $\frac{1}{\sigma_{i}}$ for $\sigma_{i}=0$ with the zero and the inverse is called Pseudo-inverse $\mathbf{R}^{+}$given as

$$
\mathbf{R}^{+}=\mathbf{V S}^{+} \mathbf{U}^{\mathrm{T}}
$$

It is important to mention that $\mathbf{R} \mathbf{R}^{+}$needs not to be the general identity matrix, which means that $\mathbf{R}^{+}$is the inverse of a modified or conditioned $\mathbf{R}$ in which the singular values smaller than a threshold are effectively removed.

\section{Corrector-strength calculation using SVD}

In case of ORM, where $M$ and $N$ are the number of BPMs and correctores, for a given perturbed orbit vector $\mathbf{w}$, the required corrector-strengths vector $\Theta$ (combining Eq. 2.22 and Eq. 2.37) can be written as,

$$
\boldsymbol{\Theta}_{w}=\mathbf{V S}^{+} \mathbf{U}^{\mathrm{T}} \mathbf{w}
$$

The corrector-strengths required to compensate the exiting orbit distortion $\mathbf{w}$ is given by $-\boldsymbol{\Theta}_{w}$.

The real space orbit distortion vector $\mathbf{w}$ and the corrector-strength vector $\boldsymbol{\Theta}_{w}$ can be transformed into SVD mode space (represented by the superscript ${ }^{m}$ ) as,

$$
\begin{aligned}
& \mathbf{w}^{m}=\mathbf{U}^{\mathrm{T}} \mathbf{w} \\
& \boldsymbol{\Theta}_{w}^{m}=\mathbf{V}^{\mathrm{T}} \boldsymbol{\Theta}_{w}
\end{aligned}
$$

and Eq. 2.38 can be written in mode space as,

$$
\Theta_{w}^{m}=\mathbf{S}^{+} \mathbf{w}^{m}
$$

Following implications can be made from the representation of orbit and corrector-strength vectors in the mode space of the ORM in Eq. 2.39 and 2.40, 
- Singular value decomposition provides the information of the mode structure of the perturbed closed orbit for a given ORM as the columns of $\mathbf{U}$. Any mode structure that does not couple to the $\mathbf{U}$ matrix i.e. for which the product $\mathbf{u}_{i} \mathbf{w}=0$ (where $i$ is the column index) cannot be corrected using the given ORM.

- The SVD singular values are the measure of the coupling sensitivity between BPM and corrector mode space, defined by the Eq. 2.39. The higher the singular values, the smaller is the required corrector-strengths in order to create/diminish the corresponding mode in BPM space.

- A unique singular value between each pair of BPM and corrector space modes, provides possibility to control/manipulate each BPM mode independent of the rest of modes.

- The modes coupled through smaller singular values require higher corrector-strengths and can cause the corrector current saturation.

- Since, the rank of the ORM and hence non-zero singular values are equal to $\min \{m, n\}$, and the dimension of the corrector space is closely related to the number of correctors, the number of correctable modes cannot be more than the number of correctors even if BPM space has larger dimension due to $m>n$.

\subsection{Closed orbit feedback system}

So far we have discussed the methods for the calculation of corrector-strengths for a given perturbed closed orbit sampled at the BPM locations. In modern synchrotrons, the orbit correction is performed in an automatic way, where the beam position is continuously measured at specific time steps and fed to a computer or FPGA hardware which is programmed for the calculation of corrector-strengths to apply at the beam. Such an orbit correction system can remove the effect of dynamic magnetic field errors from the closed orbit. The continuous readout of the beam position with the help of BPMs makes possible to "feedback" the effect of previously applied corrections, to the hardware which "controls" the corrector-strengths, hence forming the closed orbit feedback control loop.

Feedback control has very ancient history from the time of Egyptians who used it in the water clock more than 2000 years ago [44]. A relatively recent example is the feedback control that allowed James Watt [45] to invent the governor which regulated the speed of steam engines in the $19^{\text {th }}$ century. But it was only in the 1930's that a theory of feedback control was first developed by Black and Nyquist at Bell Labs. They were studying feedback as a means to linearize repeater amplifiers for telephone lines, but they had problems with what they called "singing". This was simply the onset of closed-loop instability when the feedback gain was set too high, transforming the amplifier into an oscillator. Nowadays, it is as hard as impossible to find any technologically advanced engineering process without feedback control e.g. aerospace, process control, electronics and manufacturing automation etc. [46].

The main job of any kind of the feedback system can be classified into two broad categories in terms of final requirement, 
- Set-point tracking (servo problem): Example being the Cruise control on road vehicle in order to achieve the desired set speed by the driver, without delay or overshoot, by adjusting the power output of the vehicle's engine.

- Disturbance rejection (regulator problem): In this kind of feedback control, the focus is to reject the effect of external disturbances on the time behavior of a physical system.

At this point, it is beneficial to introduce some fundamental concepts of the control theory which will be later used to characterize the closed orbit feedback system. The following reference books are used to write the upcoming subsection 46, 47].

\subsubsection{Fundamentals of control theory}

Any feedback control loop has two basic subsections i.e. the system and the controller. The system is the physical device whose dynamical behavior needs to be modified with reference to a set value or against external perturbations. The controller is an electronics device (computer or FPGA) whose output is the input to the system.

Control theory provides a mathematical recipe to model the dynamical behavior of system and hence the possibility to better design the controller for the desired behavior of the system. It also provides the tools to analyze the system response to a large variety of input signals.

\section{Linear time-invariant (LTI) dynamical system}

A system whose behavior changes over time, often in response to external stimulation or force, is called dynamical system. The linearity means that the input $u(t)$ and output $x(t)$ time response of such systems scale linearly with each other i.e. if the input is changed by a factor $a$, the output also changes with the same factor as $a x(t)$. The time invariance is the trait of a system to have the same output response if an input is applied now or $T$ seconds later except for a time delay of $T$ seconds i.e. for input $u(t-T)$, the output is $x(t-T)$. The input-output relationships for such systems is governed by the linear ordinary differential equations and most of the physical systems can be approximated as LTI systems.

One example of such systems is the simple RC series circuit with a DC source of emf $\xi$ as shown in Fig. 2.4. When the switch $\mathrm{S}$ is connected to point " $a$ ", the capacitor $C$ is charged by electromotive force (emf $\xi$ ) through the resistor $R$ and the rate of change of charge $q$ in the capacitor is given by the following differential equation as,

$$
R \frac{d q}{d t}+\frac{q}{C}=\xi
$$

Another example is the driven harmonic mass-spring oscillator with damping, whose equation of motion is given as,

$$
\frac{d^{2} x}{d t^{2}}+2 \zeta \omega_{0} \frac{d x}{d t}+\omega_{0}^{2} x=u(t)
$$

where $x(t)$ is the displacement, $\omega_{0}=\sqrt{\frac{k}{m}}$ is the undamped angular frequency of the oscillator and $\zeta=\frac{c}{2 m k}$ is called the damping ratio. $c$ and $k$ are the damping coefficient and the spring constant, respectively. $u(t)$ is the input to the system. The schematic of such a system is shown in Fig. 2.5. 


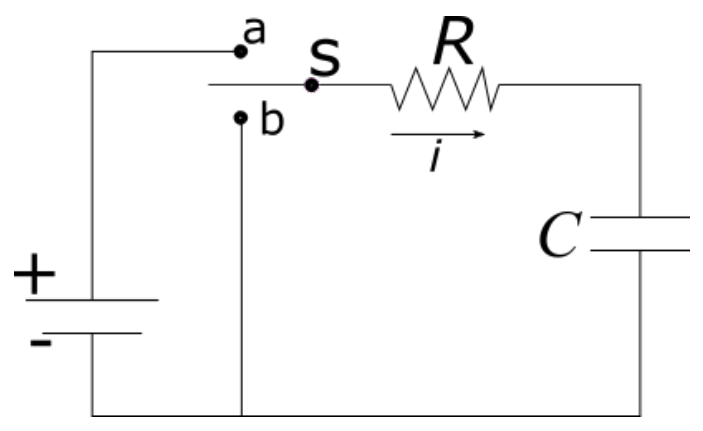

Figure 2.4 A simple RC circuit. When the switch $\mathrm{S}$ is connected to a, the capacitor is charged by the emf $\xi$ through the resistor $R$.

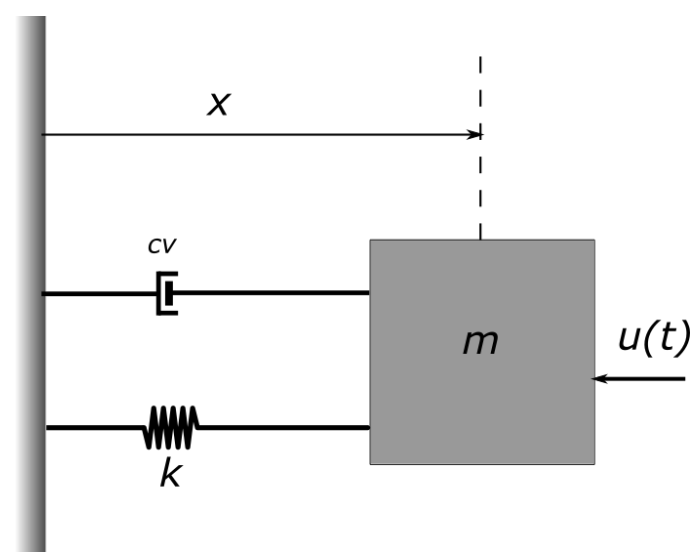

Figure 2.5 Mass-spring system with damping proportional to the velocity $v=\frac{d x}{d t}$ and arbitrary external input $u(t)$.

\section{State of a dynamic system}

The state of a dynamical system is a collection of variables that completely characterize the behavior of a system at a given time as well as for the purpose of predicting the future. The set of all possible states is called state space. In the example of mass-spring system of Eq. 2.42, $x$ and $v=\frac{d x}{d t}$ are the state variables.

\section{Response of an LTI system to an arbitrary input}

The time response of a dynamical system to an input $u(t)$ can be calculated as the solution of the linear differential equations describing the process. Let us start with the impulse response $h(t)$ of a linear system at time $t$ to an impulse at time $\tau$. This can be written as,

$$
h(t)=H\left(\delta_{\tau}\right)
$$

where the impulse $\delta_{\tau}$ is a function of time such that it has non-zero value only at $t=\tau$ and $H$ is the response operator. In order to find the output of the linear system $y(t)$ to an arbitrary 
input signal $u(t)$, let us write the input signal as sample of itself i.e.

$$
u(t)=\int_{-\infty}^{\infty} u(\tau) \delta_{\tau} d \tau
$$

This means that $u(t)$ can be written as a weighted integral of $\delta$ functions. The response of the linear system $H(u(t))$ to the input can be written as,

$$
y(t)=H(u(t))=H\left(\int_{-\infty}^{\infty} u(\tau) \delta_{\tau} d \tau\right)
$$

Due to the linearity of the system, the order of the operator $H$ and integration can be interchanged as,

$$
y(t)=\int_{-\infty}^{\infty} u(\tau) H\left(\left(\delta_{\tau}\right)\right) d \tau
$$

Substituting the definition of impulse response from Eq. 2.43 in the above equation,

$$
y(t)=\int_{-\infty}^{\infty} u(\tau) h(t) d \tau
$$

Equation 2.47 is the convolution integral and the output $y(t)$ of the system to input $x(t)$ can be defined using the convolution operator * as,

$$
y(t)=u(t) * h(t)
$$

In order to demonstrate the above definition of the output response, the system of series RC circuit shown in Fig. 2.4 is considered. The homogeneous solution of the Eq. 2.41 for zero initial conditions can be written as,

$$
q(t)=A_{0} e^{-\frac{t}{R C}}
$$

which can be verified by the direct substitution. In order to solve for the total response, let the undetermined coefficient be a function of time as,

$$
q(t)=A(t) e^{-\frac{t}{R C}}
$$

Substituting Eq. 2.50 into Eq. 2.41 one can solve for the input $u(t)=\xi(t) C$ as,

$$
R C\left(A^{\prime}(t) e^{\frac{-t}{R C}}-\frac{1}{R C} A(t) e^{\frac{-t}{R C}}\right)+A(t) e^{\frac{-t}{R C}}=u(t)
$$

Simplifying,

$$
A^{\prime}(t)=u(t)\left(\frac{1}{R C} e^{\frac{t}{R C}}\right)
$$

which can be integrated from $t=0$ to get

$$
A(t)=\int_{0}^{t} u(\tau)\left(\frac{1}{R C} e^{\frac{t}{R C}}\right)+A(0)
$$


Then

$$
\begin{aligned}
q(t) & =A(t) e^{-\frac{t}{R C}} \\
& =e^{\frac{-t}{R C}} \int_{0}^{t} u(\tau)\left(t / R C e^{\frac{t}{R C}}\right)+A(0) e^{\frac{-t}{R C}} \\
& =\int_{0}^{t} u(\tau)\left(\frac{1}{R C} e^{(t-\tau) / R C}\right)+A(0) e^{\frac{-t}{R C}}
\end{aligned}
$$

where the first term on the right hand side is the response of the $\mathrm{RC}$ circuit to the input $u(t)$ which can also be calculated using the impulse response $h(t)$ of the system as follows,

$$
h(t)=\frac{1}{R C} e^{-t / R C}
$$

The response of the system to an input $u(t)$ is then

$$
\begin{aligned}
q(t) & =\int_{-\infty}^{\infty} u(\tau) h_{\tau}(t) d \tau \\
& =\int_{-\infty}^{\infty} u(\tau)\left(\frac{1}{R C} e^{(t-\tau) / R C}\right) d \tau
\end{aligned}
$$

Hence demonstrated that Eq. 2.48 can be used to transform any input to the system to the output, once the impulse response $h(t)$ of the system is known and it is the most fundamental basis of the control theory.

\section{Transfer function}

Although the final goal of the mathematical treatment of the system models is to predict the time behavior of the systems for various input signals, dealing with linear differential equations in time domain gets cumbersome with the increasing order of the system as well as the number of state variables. For example, when the output of one LTI system is the input of another LTI system and so on. On the other hand, dealing in the frequency domain is more useful e.g. solving the differential equations in the Laplace domain defined as

$$
\mathscr{L}(y(t))=Y(s)=\int_{0}^{\infty} y(t) e^{-s t} d t
$$

where the complex parameter $s=\sigma+j \omega$ defines the $\mathscr{L}$-space with the following very useful properties of Laplace transform,

$$
\begin{aligned}
\mathscr{L}(a y(t)) & =a \mathscr{L}(y(t))=a Y(s) \\
\mathscr{L}\left(a y_{1}(t)+b y_{2}(t)\right) & =a \mathscr{L}\left(y_{1}(t)\right)+b \mathscr{L}\left(y_{2}(t)\right)=a Y_{1}(s)+b Y_{2}(s) \\
\mathscr{L}(y(t-\tau)) & =e^{-\tau s} Y(s) \\
\mathscr{L}\left(\frac{d y(t)}{d t}\right) & =-y(0)+s Y(s) \\
\mathscr{L}\left(\int y(t) d t\right) & =\frac{1}{s} Y(s) \\
\left.\mathscr{L}\left(y_{1}(t) * y_{2}(t)\right)\right) & =Y_{1}(s) Y_{2}(s)
\end{aligned}
$$


Let us consider a linear input-output system described by the generalized differential equation including derivatives for both input $u(t)$ and output $y(t)$ as,

$$
\frac{d^{n} y(t)}{d t^{n}}+a_{1} \frac{d^{n-1} y(t)}{d t^{n-1}}+\ldots+a_{n} y(t)=\frac{d^{m} u(t)}{d t^{m}}+b_{1} \frac{d^{m-1} u(t)}{d t^{m-1}}+\ldots+a_{m} u(t)
$$

The Laplace transform on both sides of Eq. 2.59 with zero initial conditions yields,

$$
\left(s^{n}+a_{1} s^{n-1}+\ldots+a_{n}\right) Y(s)=\left(s^{n}+b_{1} s^{n-1}+\ldots+b_{n}\right) U(s)
$$

where $U(s)$ and $Y(s)$ are the Laplace transforms of the input and output signals, respectively. Rearranging Eq. 2.60 results in,

$$
\begin{aligned}
Y(s) & =\frac{\left(s^{n}+b_{1} s^{n-1}+\ldots+b_{n}\right)}{\left(s^{n}+a_{1} s^{n-1}+\ldots+a_{n}\right)} U(s) \\
Y(s) & =H(s) U(s)
\end{aligned}
$$

Eq. 2.61 is the frequency domain version of Eq. 2.48 where the transfer function $H(s)$ is the impulse response in the frequency domain. For the first and second order system of Eqs. 2.41 and 2.42 , the transfer functions can be defined as,

$$
\begin{aligned}
& H_{1}(s)=\frac{K_{0} \omega}{s+\omega_{0}} \\
& H_{2}(s)=\frac{K_{0} \omega_{0}}{s^{2}+2 \zeta \omega_{0} s+\omega_{0}^{2}}
\end{aligned}
$$

where $K_{0}$ is introduced to normalize and generalize the definition.

The transfer function representation of an LTI system provides very fruitful insight into the physical behavior of the system. Important points are summarized below.

- Transfer function is the ratio of Laplace transform of output to Laplace transform of input assuming all initial conditions to be zero.

- The transfer function of a system is the Laplace transform of its impulse response under assumption of zero initial conditions.

- Replacing ' $s$ ' variable with linear operation $D=\frac{d}{d t}$ in transfer function of a system, the differential equation of the system can be obtained.

- The transfer function of a system does not depend on the inputs to the system.

- The roots of the numerator and denominator of the transfer function called system zeros and poles, respectively, provide direct information of the stability of the system (explained below).

The zero frequency gain of a system is given by the magnitude of the transfer function at $s=0$. It represents the ratio of the steady state value of the output with respect to a impulse 
input. Generally, any transfer function of an LTI system can be represented in the form of polynomial fraction as,

$$
H(s)=\frac{\prod_{i=0}^{n}\left(s+z_{i}\right)}{\prod_{i=1}^{n}\left(s+p_{i}\right)}
$$

where $z_{i}$ are called the zeros and $p_{i}$ are called the poles of the transfer function. Since, the transfer function completely represents the differential equations of the system, its poles and zeros effectively define the system response. For example, applying the Laplace transform method for the transient solution of Eq. 2.42 for $u(t)=0$ and for $x(0)=0$ and $x^{\prime}(0)=v_{0}$ one can obtain,

$$
X(s)=\frac{v_{0}}{s^{2}+2 \xi \omega_{0}+\omega_{0}^{2}}=\frac{v_{0}}{\left(s+p_{1}\right)\left(s+p_{2}\right)}
$$

where $p_{1,2}=-\xi \omega_{0} \pm \omega_{0} \sqrt{\xi^{2}-1}$ which can be real or complex depending upon the value of $\xi$. The solution $x(t)$ can be calculated by the inverse Laplace transform of Eq. 2.64 to be

$$
x(t)=\sum C_{i} e^{p_{i} t}
$$

where $C_{i}$ are the constants. One can see that the transient (homogeneous) response of the system is completely defined by the poles for the LTI systems. For example, for the under-damped case of spring-mass system, $0<\xi<1$ and $p_{1,2}=\sigma \pm j \omega$ where $\sigma=-\xi \omega_{0}$ and

$\omega=\sqrt{1-\xi^{2}}$. The real part represents the amplitude growth or damping and the imaginary part defines the oscillation frequency, respectively as,

$$
x(t)=\sum C_{i} e^{\sigma t}(\cos (w t) \pm j \sin (w t))
$$

If a system has several poles, the poles closest to the imaginary axis define the main behavior of the system and are referred to as the dominant poles.

\section{System stability}

The poles of the transfer function give direct insight about the stability of the system regardless of the input type. By definition, an $n^{\text {th }}$ order linear system is asymptotically stable only if all of the components in the homogeneous response from a finite set of initial conditions decay to zero as time increases. If any pole has a positive real part, there is a component in the output e.g. given in Eq. 2.65 that increases without bound, causing the system to be unstable. In order for a system to be stable, all of its poles must have negative real parts, i.e. they must all lie within the left-half of the complex s-plane shown in Fig. 2.6. All other cases are also depicted with the help of time responses for each pole position. A system having one or more poles lying on the imaginary axis of the complex $s$-plane has non-decaying oscillatory component in its homogeneous response, and is defined to be marginally stable. 


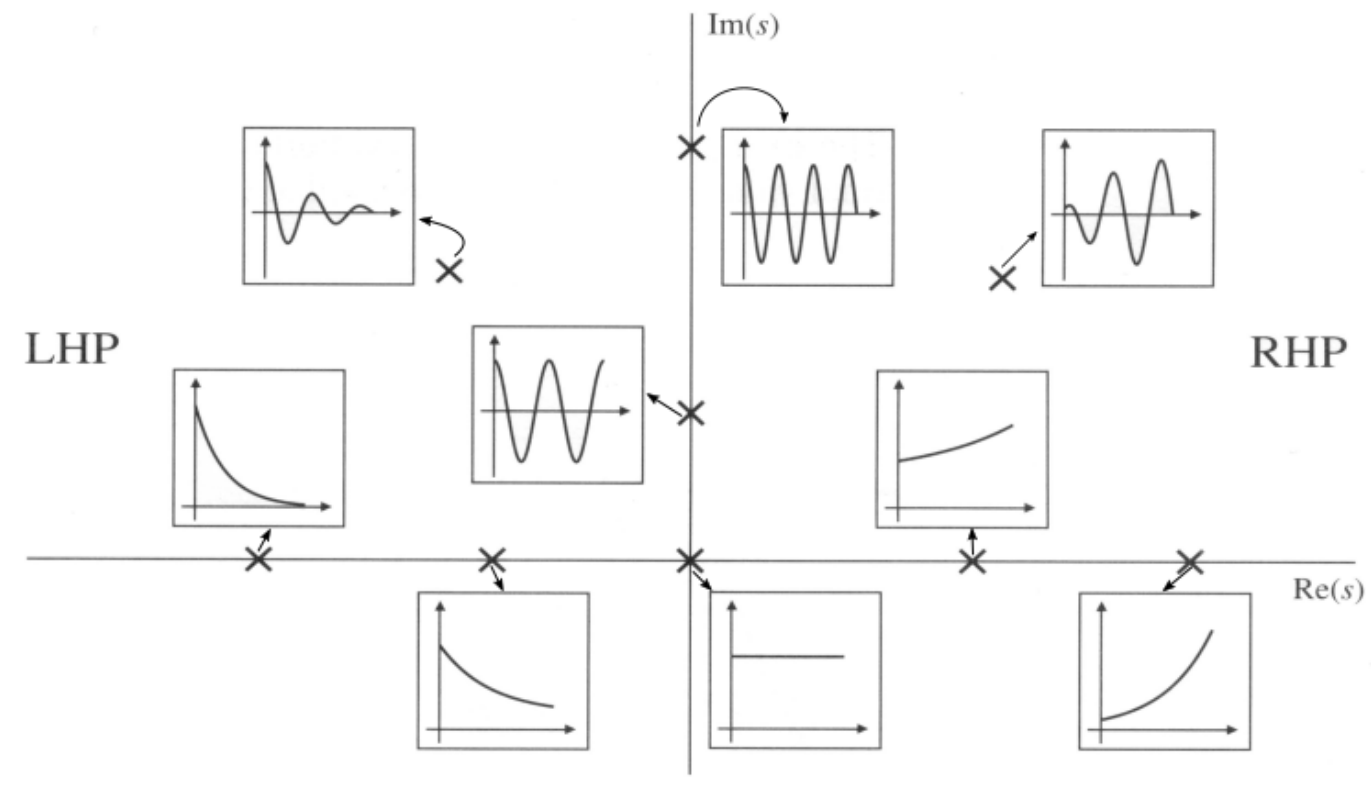

Figure 2.6 Transient response of linear systems associated with certain pole location marked as ' $\mathrm{x}$ '. Stable systems are required to have poles located in the left-half plane (LHP), whereas unstable systems have poles in right-half plane of the complex $s$-plane 48$]$.

\section{Frequency response (Bode plot)}

Another important description is the system's response with respect to the sinusoidal excitation of constant amplitude and phase but varying frequency. The output response represented in two separate quantities i.e. the magnitude (gain) and the phase of the output with respect to the input signals plotted as a function of the frequency of excitation is called Bode plot. Such an output response can be measured by exciting the system over a frequency range and recording the output response. Bode plots are used with the Fourier Transform of a given system instead of Laplace Transform in order to work in the pure frequency domain. Frequency response can be evaluated with the help of transfer function by the change of variable from Laplace variable " $s$ " to Fourier variable " $j \omega$ " i.e.

$$
H(j \omega)=\frac{Y(j \omega)}{U(j \omega)}
$$

which is effectively the transfer function evaluated on the imaginary axis of the complex $s$-plane. The magnitude and the phase of the response to the sinusoidal inputs at each fixed frequency for Eq. 2.67 can be calculated as,

$$
\begin{aligned}
M\left(Y_{B}(j \omega)\right) & =20 \log _{10}(|H(j \omega)|) \\
\phi\left(Y_{B}(j \omega)\right) & =\arg (H(j \omega))
\end{aligned}
$$




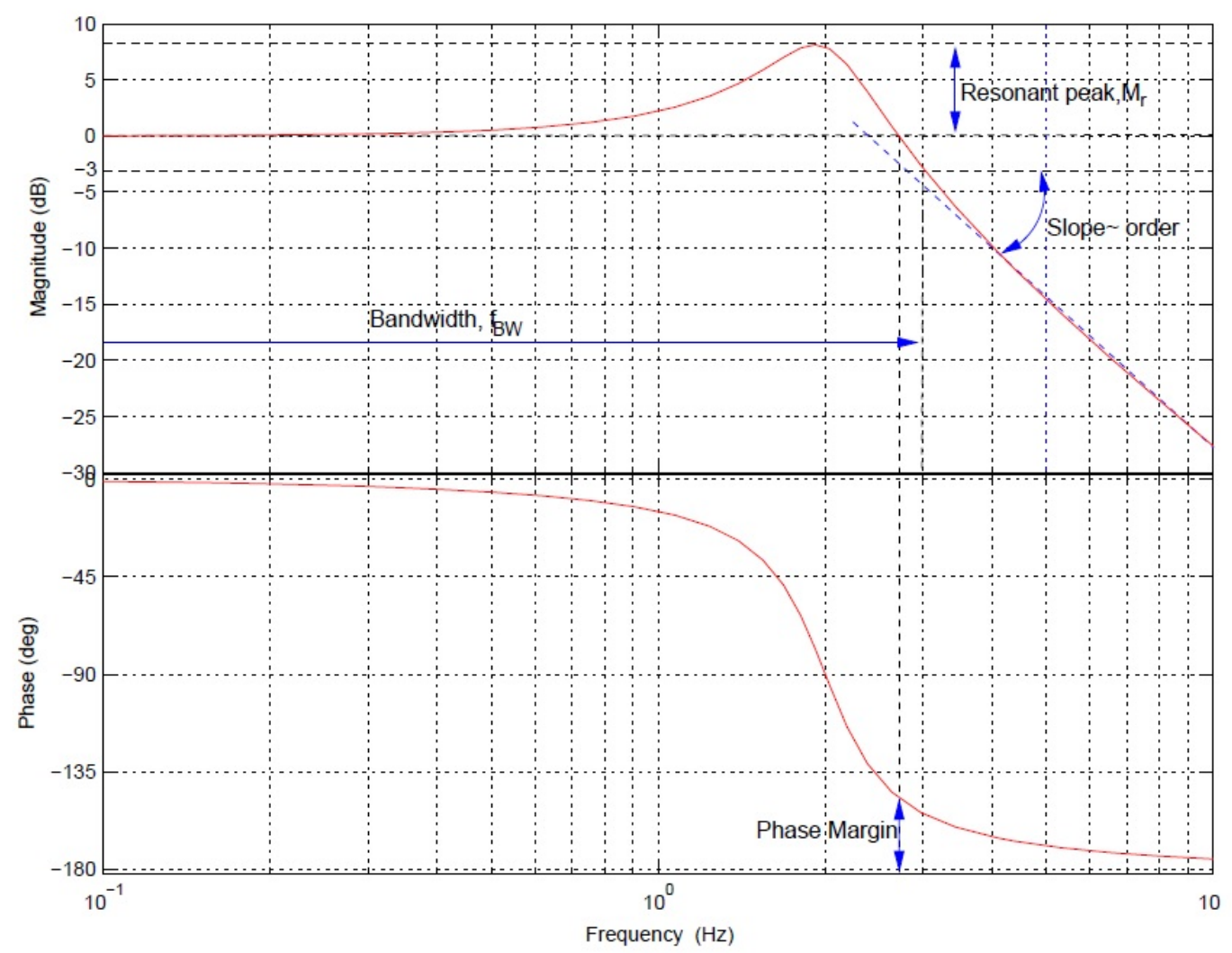

Figure 2.7 Example of a typical Bode plot for a second order system $36 \mid$.

The magnitude $M\left(Y_{B}\right)$ and phase $\phi\left(Y_{B}\right)$ are usually plotted as a function of frequency $f=\frac{\omega}{2 \pi}$ and measured in units of decibels $(\mathrm{dB})$ and degree, respectively. Figure 2.7 shows the typical Bode plots for a second order system. The $\mathrm{x}$-axis is logarithmic in frequency with the base 10. Often the unit "decade" is used to describe the distance between the two frequencies $f_{1}$ and $10 f_{1}$. The maximum phase lag depends on the order $n$ of the system and is given by,

$$
\delta \phi_{\max }=n \frac{\pi}{2}
$$

Another indication for the order of the system is the slope indicated in the Bode plot which is proportional to $20 n \frac{d B}{\text { decade }}$. Following important information can be extracted from a Bode plot (see Fig. 2.7):

- The Bandwidth $f_{B W}$ which is defined by the frequency where the gain drops below $-3 \mathrm{~dB}$.

- The resonant peak $M_{r}$ which is the highest value of the frequency response and an indication for the system's damping coefficient: the lower the damping, the higher the resonant peak.

- The phase margin $\Delta \phi$ is given as the difference between the systems phase lag and $\pi$ $\left(180^{\circ}\right)$ at the frequency when the gain crosses $0 \mathrm{~dB}$. 


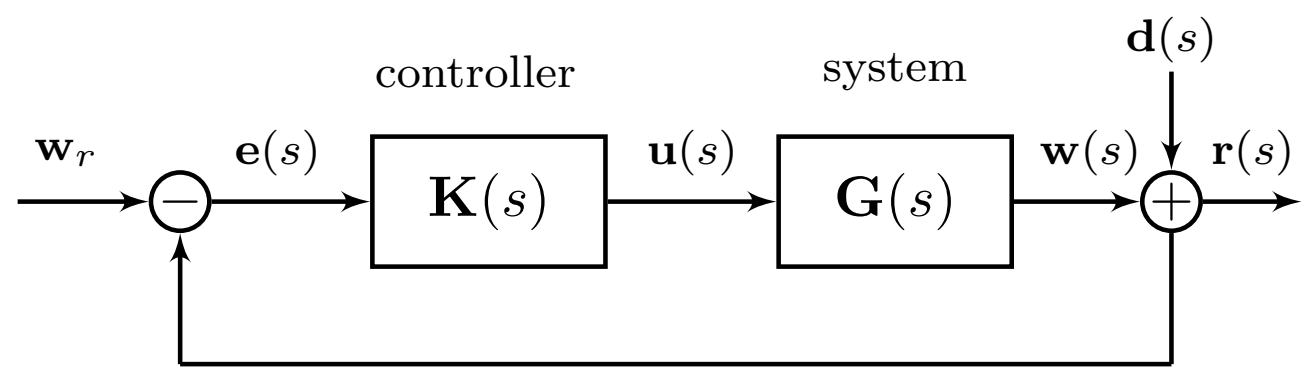

Figure 2.8 Schematic diagram of a basic feedback loop.

- Similarly one defines the gain margin $\Delta M$ as the difference of the magnitude with respect to zero in the logarithmic representation at a frequency where the phase margin is $\Delta \phi=0$.

\subsubsection{Feedback loop}

A typical feedback loop is shown in Fig. 2.8 where the transfer functions of LTI systems are represented as blocks and interconnected in the order of signal flow. Here we directly talk about Multiple-Input-Multiple-Output (MIMO) control problem where the input to the system is a vector $\mathbf{u}(s)$ of size $M \times 1$ and the output is also a vector of size $N \times 1$ while the transfer function is an $M \times N$ matrix whose each element is a Single-Input-Single-Output (SISO) transfer function. For both requirements from a feedback system (servo and regulator problems), a controller is used to manipulate the input to the system $\mathbf{G}(s)$ such that the error $\mathbf{e}(s)$ between the set value $\mathbf{w}_{r}$ and the system output $\mathbf{r}(s)$ remains small in spite of disturbances $\mathbf{d}(s)$. The error signal, closed-loop output and controller output are defined by the following matrix properties as,

$$
\begin{aligned}
\mathbf{r}(s) & =\mathbf{G}(s) \mathbf{K}(s) \mathbf{e}(s)+\mathbf{d}(s) \\
\mathbf{e}(s) & =\mathbf{w}_{r}(s)-\mathbf{r}(s) \\
\mathbf{u}(s) & =\mathbf{K}(s) \mathbf{e}(s)
\end{aligned}
$$

Following three important transfer function are defined for the closed-loop as,

$$
\begin{aligned}
\mathbf{L}(s) & =\mathbf{G}(s) \mathbf{K}(s) \\
\mathbf{S}(s) & =(\mathbf{I}+\mathbf{G}(s) \mathbf{K}(s))^{-1} \\
\mathbf{T}(s) & =(\mathbf{I}+\mathbf{G}(s) \mathbf{K}(s))^{-1} \mathbf{G}(s) \mathbf{K}(s) \\
& =(\mathbf{I}+\mathbf{L}(s))^{-1} \mathbf{L}(s)
\end{aligned}
$$

where $\mathbf{L}(s)$ is called the loop transfer function, $\mathbf{S}(s)$ is the sensitivity function from disturbance $\mathbf{d}(s)$ to the output $\mathbf{r}(s)$ and $\mathbf{T}(s)$ is the complementary transfer function from set value $\mathbf{w}_{r}(s)$ to the output $\mathbf{r}(s)$. All the fundamental tools of the control theory discussed in the previous subsection can be applied on the closed-loop transfer functions e.g. Bode plot representation of sensitivity function $\mathbf{S}(s)$ provides the information about the frequency range of effective disturbance rejection and is very useful tool during the controller design process. 


\section{Effect of feedback on poles and zeros}

The comparison between open- and closed-loop transfer functions is made between $\mathbf{G}(s)$ and $\mathbf{T}(s)$ of Eq. 2.71. The location of poles and zeros of the closed-loop transfer function relative to the open-loop poles and zeros depends upon the controller gains. Following conclusions are given without proof 47 ,

- The zero locations are unchanged by the feedback between $\mathbf{G}(s)$ and $\mathbf{T}(s)$.

- The pole locations are changed by the feedback as a function of controller gain. If the controller gain approaches zero, the poles of $\mathbf{T}(s)$ approach to the poles of $\mathbf{G}(s)$. On the other hand, if the controller gain gets larger (approaching to infinity), the closed-loop poles move from open-loop poles to the open-loop zeros. The open-loop zeros on the right-hand side of the complex plane therefore imply high gain instability of the closed-loop.

\subsubsection{System model for closed orbit feedback (COFB) system}

Closed orbit correction using a feedback system is a MIMO control problem due to $M$ correctors and $N$ BPMs. The system model can be described in two separate parts i.e. the spatial part and the temporal parts. The spatial part is the real valued orbit response matrix (ORM) defined by Eq. 2.23. In case of SIS18, the maximum size of the ORM is $12 \times 12$. The temporal part represents the frequency response of BPM electronics, corrector magnet coils, their power supplies, vacuum chamber and cables etc. The temporal response of all hardware components can be represented as one separate transfer function in the continuous frequency domain as $g(s) e^{-\tau_{d} s}$ (where $s$ represents the Laplace domain variable and $\tau_{d}$ is the pure delay in seconds) resulting in the total process model as,

$$
\mathbf{G}(s)=g(s) e^{-\tau_{d} s} \mathbf{R}
$$

Here, it is assumed that all corrector magnets and BPMs have same temporal responses, respectively, which is a realistic assumption in most of the cases. On the other hand, $g(s)=\prod g_{i}(s)$ where $g_{i}$ represents the transfer function of individual subsystems of the closed-loop, briefly discussed below.

\section{Corrector magnets}

The corrector magnets are small dipole magnets used to steer the beam at discrete locations of choice and act as the actuators in the closed-loop of the COFB system. The corrector magnets are artificial sources of dipolar field errors which are used to compensate the effect of the non-removable sources of the similar field errors in the ring. They are also used for the alignment of the beam at the injection and extraction to and from the ring as well in the transfer lines. In SIS18, the vertical correctors have dedicated positions in the ring while the horizontal correctors are the extra winding on the main dipoles.

Since, the inductive and resistive parts of the electromagnets as well as the hysteresis losses and eddy currents in the magnet core can be modelled as low pass RL series filter which 
can have a limiting effect on the achievable bandwidth of the feedback system. The transfer function model for such a circuit is

$$
\frac{1}{a_{L} s+a_{R}}
$$

where $a_{L}$ is the magnet inductance and $a_{R}$ is the magnet resistance.

\section{Power converters}

Power converters are complicated circuits and are the potential source of higher order transfer functions in the closed orbit feedback loop due to the presence of many solid state hardware.

\section{Vacuum chamber}

Vacuum chamber contributes in the overall frequency response as a low pass filter due to the eddy current losses in the stainless steel walls. SIS18 vacuum chambers are of elliptical type with the ratio of minor to major diameter $\left(\frac{2 b}{2 a}\right)^{2}=0.1$ and the transfer function for such a geometry is worked out to be 49

$$
\frac{1}{a_{v a c} s+1}
$$

where $a_{v a c}=\frac{1.46}{2} v_{\text {per }} v_{\text {con }} a_{\text {rad }} a_{\text {wall }}$ and for vacuum permeability $v_{v a c}=4 \pi \times 10^{-7} \mathrm{H} \cdot \mathrm{m}^{-1}[50$ ], 316LN stainless steel conductivity $v_{c o n}=1.35146(\Omega \mathrm{m})^{-1}[3]$, the major radius $a_{\text {rad }}$ of $0.1907 \mathrm{~m}$ and wall thickness of $400 \mu \mathrm{m}[51], a_{v a c}=94.51 \mu \mathrm{s}$ and consequently the cut-off frequency for such a low pass filter is much higher of the order of $10 \mathrm{kHz}$ as compared to the range of interest for closed orbit correction (several hundred $\mathrm{Hz}$ ).

It is important to mention that the measurement of the collective transfer function $g(s)$ is always recommended and the process is called full-system identification.

\section{Beam position monitors (BPMs)}

Beam position monitors serve the job of sensors in the closed orbit feedback system and their types and shapes vary for electron and hadron synchrotrons. BPMs work on the principle of comparison of induced voltage on two parallel plates around the beam in each plane [52]. The analog signal from each plate for each bunch is fed to the digitizer and converted into the transverse bunch position at the bunch revolution frequency in the ring. Beam position sensitivity is the measure of proportionality between the beam's transverse displacement and the difference of voltage induced on both plates.

SIS18 BPMs are of so-called linear cut-type which is a unique box-like device with longitudinal length of $0.155 \mathrm{~m}$. Each plate is terminated with a high impedance amplifier (details are given in [53]). Such a BPM geometry gives the beam position readout independent of the beam size and helps to achieve a linear relation between the beam position and the induced image voltages $(U)$ on the left and right plates as,

$$
w=\frac{1}{S_{w}} \frac{U_{\text {right }}-U_{\text {left }}}{U_{\text {right }}+U_{\text {left }}}
$$



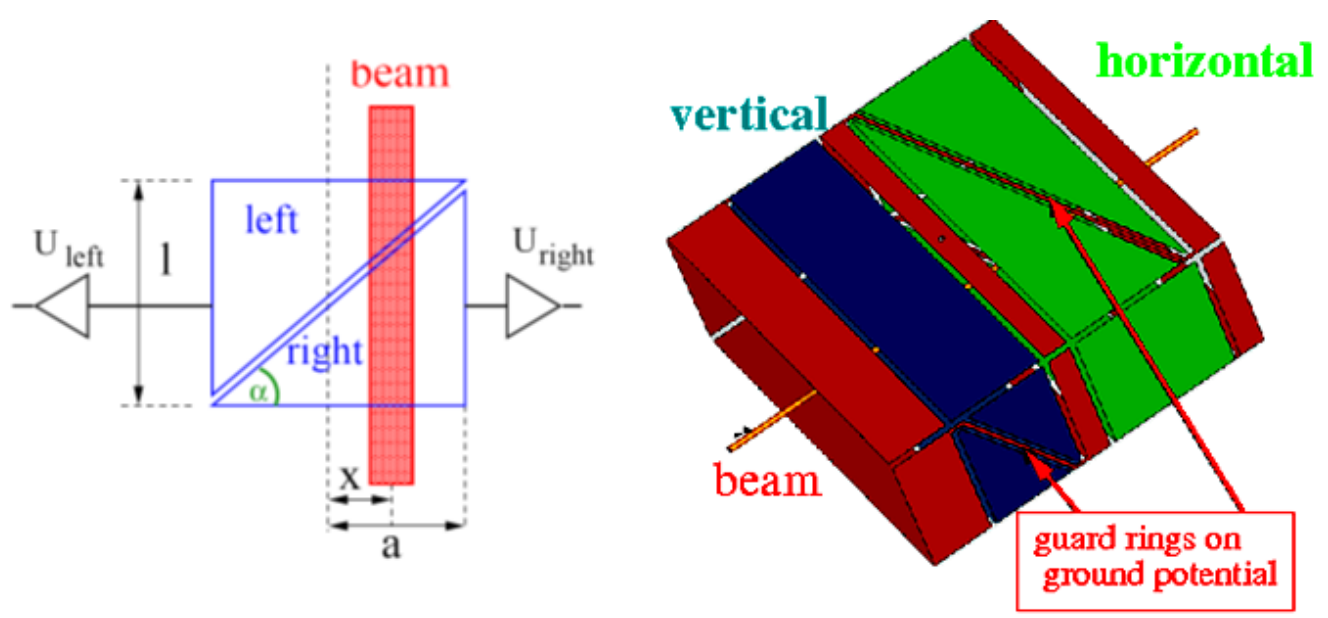

Figure 2.9 Left: Scheme of the position measurement using the so-called linear cut BPM and an example of an electrode arrangement for the horizontal plane. Right:

Schematic drawing for a linear cut BPM for both planes [54].

where $S_{w}$ is the position sensitivity in either plane. The sensitivity for the SIS18 BPM geometry shown in Fig. 2.11 has been calculated in [54] as,

$$
S_{w}=\frac{1}{a_{w}}
$$

where $a_{w}$ is the half-length of the gap between the plates in the plane of measurement. For the horizontal plane of SIS18, $S_{x}=\frac{1}{100} \% / \mathrm{mm}$.

Similarly, the resolution defined as the ability for measuring small displacement variations has been shown to be less than $100 \mu \mathrm{m}$ in [55, 56] for SIS18 BPMs. Therefore, the SIS18 BPMs are well suited for their use as sensors in the SIS18 COFB system.

\section{Hardware for controller implementation}

The controller hardware is considered here as a digital device either a computer, digital signal processors (DSP) or field programmable gate arrays (FPGA) and can contribute in the pure delay depending upon the time required for the corrector-strength calculation, in terms of the digital clock frequency of the digital controller. The calculation time depends strongly on the size of the orbit response matrix and the choice of the hardware is made accordingly.

\subsubsection{Controller}

As discussed in previous section, the system model for a closed orbit feedback can be separated into the spatial and temporal parts with the approximation that each corrector channel has the same frequency response $g(s)$. Consequently, the controller can also be modeled as having two separate parts. The spatial part is the pseudo-inverse $\mathbf{R}_{\Theta}^{+}$((Eq. 2.37)) of the ORM $\mathbf{R}$, for the corrector-strength calculation. The subscript $\Theta$ is used to distinguish the controller 
model ORM from the actual system ORM R. The temporal part $k(s)$ depends upon the system model $g(s)$ and the the total controller model can be written as,

$$
\mathbf{K}(s)=k(s) \mathbf{R}_{\Theta}^{+}
$$

\section{Proportional Integral (PI) controller}

PI controller is the mostly used type of controller in COFB systems and has a first order transfer function between error signal $e(s)$ and the controller output $u(s)$ and is given in Laplace domain as,

$$
k(s)_{P I}=\frac{u(s)}{e(s)}=K_{p}+\frac{K_{i}}{s}
$$

where $K_{p}$ and $K_{i}$ are called the proportional and integral gains, respectively, which need to be tuned. Eq. 2.78 can be written in time domain using the relation given in Eq. 2.58 as,

$$
u(t)=K_{p} e(t)+K_{i} \int e\left(t^{\prime}\right) d t^{\prime}
$$

The integral part unlike the proportional term increases the controller output signal $u(t)$ until the error signal $e(t)$ is zero and thus minimizes any steady state error. For practical implementations, the correctors can have finite strengths and increase in the controller output $u(t)$ can result in the saturation of the correctors. In order to avoid this saturation, an anti-windup scheme is used such that whenever the PI controller output reaches a certain limit defined by the user, the Integrator accumulator is held constant at the last stored values.

\section{Internal model controller (IMC)}

IMC belongs to the class of controllers which are based upon the best known model of the system as shown schematically for a SISO system in Fig. 2.10. The idea behind the IMC-structure is that the parameter $q(s)$ can be designed in an open-loop fashion since the feedback signal only contains information about the difference between the actual output of the system $g(s)$ and the output predicted from the model $\tilde{g}(s)$.

The effective controller $k(s)_{I M C}$ can thus be calculated as,

$$
k(s)_{I M C}=q(s)(1-\tilde{g}(s) q(s))^{-1}
$$

which ensures the stability of the feedback loop if both $q(s)$ and $\tilde{g}(s)$ are stable. IMC helps to design stable feedback loop as for a stable $\tilde{g}(s)$, any choice of stable $q(s)$ can be made (chapter4 of 47]).

An ideal choice for $q(s)$ is the inverse of the best known model augmented by a low pass filter $Z(s)$ as,

$$
q(s)=[\tilde{g}(s)]^{-1} Z(s)=[\tilde{g}(s)]^{-1} \frac{p}{s+p} e^{-s \tau_{d}}
$$

which results into following IMC-controller

$$
k(s)_{I M C}=[\tilde{g}(s)]^{-1} \frac{Z(s)}{1-Z(s)}
$$




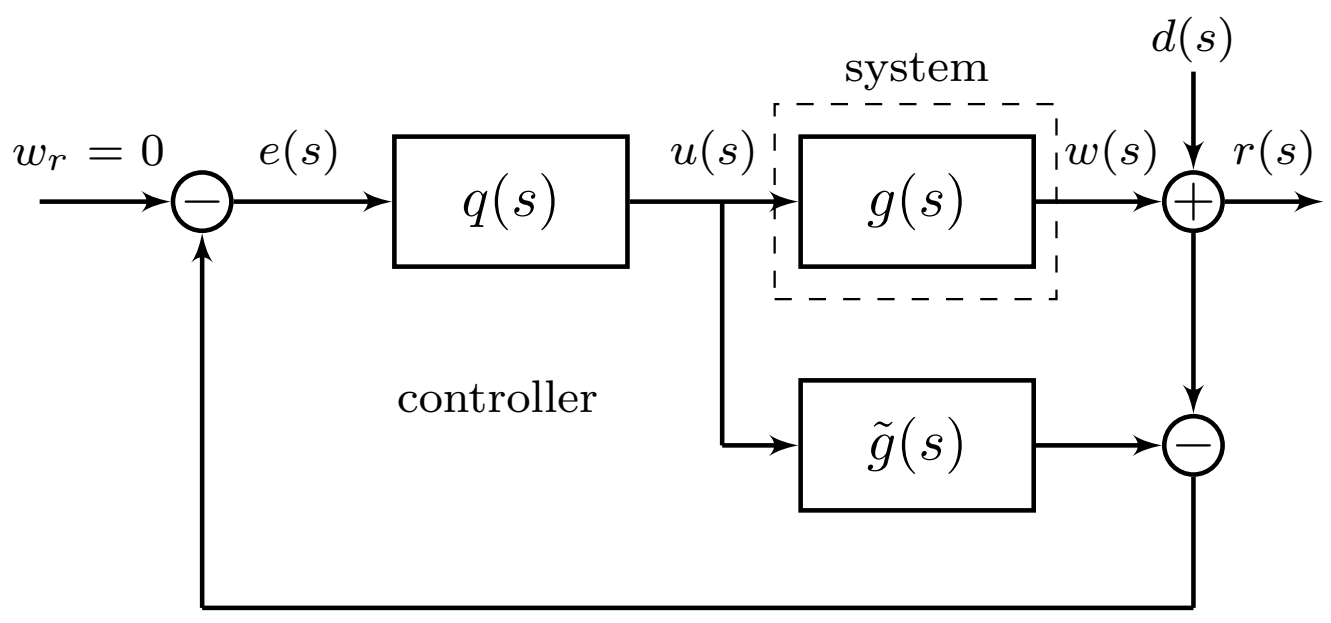

Figure 2.10 Schematic of an internal model controller (IMC) in $s$-domain for a SISO system.

\section{IMC-based PI tuning for first-order system}

For a first-order system $g(s)$ whose best known model $\tilde{g}(s)$ is given as,

$$
\tilde{g}(s)=\frac{a}{s+a} e^{-s \tau_{d}}
$$

and the low pass filter given as,

$$
Z(s)=\frac{p}{s+p} e^{-s \tau_{d}}
$$

The IMC controller can be computed as,

$$
k(s)_{I M C}=[\tilde{g}(s)]^{-1} \frac{Z(s)}{1-Z(s)}=\frac{p(s+a)}{a\left(s+p\left(1-e^{-s \tau_{d}}\right)\right)}
$$

Using, $1-e^{-s \tau_{d}} \approx s \tau_{d}$ for small $s$,

$$
k(s)_{I M C}=\frac{p(s+a)}{a s\left(1+p \tau_{d}\right)}=\frac{K_{p} s+K_{i}}{s}
$$

which results into,

$$
\begin{aligned}
K_{p} & =\frac{p}{a\left(1+p \tau_{d}\right)} \\
K_{i} & =\frac{p}{1+p \tau_{d}}
\end{aligned}
$$

and suggests that $K_{p}=\frac{K_{i}}{a}$ 


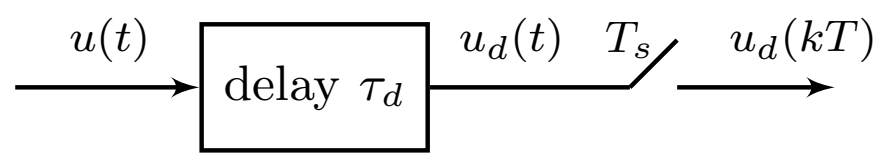

Figure 2.11 Sampling of a delayed signal.

\subsubsection{The z-transform}

In modern applications, no controller is implemented in the analog domain and digital controllers always act on discrete time steps set by the clock frequency. For example, the COFB control system is implemented in "sample and hold" mode where the beam position is measured at discrete time steps $\left\{t=k T_{s}, k \in \mathbb{Z}^{+}\right\}$with $T_{s}$ being the sampling time interval and corrector-strengths are held constant over the time interval $t \in\left\{k T_{s},(k+1) T_{s}\right\}$. On the other hand, even if almost all processes of physical systems and actuators are analog, the signal processing is done in discrete time domain with the help of analog-to-digital converters (ADC) and digital-to-analog converters (DAC). In this case, a "change of language" is advised from Laplace domain to discrete domain called the $z$-domain for digital interpretation of system models and the implementation of the controllers. Anyhow, if the clock frequency is an order of magnitude higher than the frequency of interest for the feedback system, a continuous domain analysis still provides valid conclusions. The $z$-transform can be derived from the Laplace transform with the following relation [57]

$$
z=e^{s T_{s}}
$$

where $T_{s}$ is the sampling period of a continuous signal $u(t) \rightarrow u\left(k T_{s}\right)$. With the definition given in Eq. 2.88, $z$-transform holds almost all properties of the Laplace transform given in Eq. 2.58 where the differential equations are written as difference equations for discrete domain. The only ambiguity arises for dealing with the pure delay in the $z$-transform when it lies somewhere between the sampling points in time. The $z$-transform associated with the delayed signal is known as the modified $z$-transform $\left(\mathcal{Z}_{m}\right)$ as explained below. Let us consider a casual continuous-time domain signal $u(t)$ only for $t \geq 0$, sampled every $T_{s}$ seconds. Introducing a delay $\tau_{d}<T_{s}$ before the sampler as shown in Fig. 2.11, the output of the delayed element before the sampler is,

$$
u_{d}(t)= \begin{cases}u\left(t-\tau_{d}\right), & \text { if } t \geq 0 \\ 0, & \text { if } t<0\end{cases}
$$

Delaying a causal system always results in an initial zero value. To avoid inappropriate initial values, the delay can be written for $0 \leq m<1$ as,

$$
\begin{aligned}
\tau_{d} & =T_{s}-m T_{s} \\
m & =1-\frac{\tau_{d}}{T_{s}}
\end{aligned}
$$

If $u_{-1}(t)$ is defined as $u(t)$ delayed by one complete sampling period i.e. $u_{-1}(t)=u\left(t-T_{s}\right)$ then,

$$
u_{d}(t)=u_{d}\left(t-\tau_{d}\right)=u\left(t-T_{s}+m T_{s}\right)=u_{-1}\left(t+m T_{s}\right)
$$


For the sampling of delayed signal $u_{d}(t)$ with sampling period $T_{s}$, replace $t$ with $k T_{s}$ in Eq. 2.91 as,

$$
u_{d}\left(k T_{s}\right)=u_{-1}\left(k T_{s}+m T_{s}\right), k=0,1,2, \ldots
$$

For a delay of one complete sampling period the $z$-transform of $u_{-1}(t)$ is given as,

$$
U_{-1}(z)=z^{-1} U(z)
$$

The modified $z$-transform for a signal delayed by $\tau_{d}$ can be defined by the Eqs. 2.92 and 2.93 as,

$$
U(z, m)=\mathcal{Z}\left(u_{d}\left(k T_{s}\right)\right)=z^{-1} \mathcal{Z}\left\{u\left(k T+m T_{s}\right)\right\}=\mathcal{Z}_{m}\left(u\left(k T_{s}\right)\right)
$$




\section{Chapter Three}

\section{Problem description: on-ramp fast closed orbit correction in SIS18}

In this chapter, the requirements for the SIS18 closed orbit feedback system are discussed in the light of the peculiar behaviors of SIS18 synchrotron.

\subsection{Motivation of orbit correction at SIS18}

SIS18 synchrotron has served as the main synchrotron of GSI facility at Darmstadt Germany, since 1988 and has been the contributor of several scientific discoveries by delivering high energy beams of heavy ions to various experimental facilities. The notables include FRagment Separator (FRS) and Experimental Storage Ring (ESR) [16]. The new role of SIS18 is to deliver the high intensity beams to the upcoming SIS100 synchrotron (currently under construction in 2021) of the Facility for Antiproton and Ion Research (FAIR), as the booster ring [58]. The planned intensities for FAIR project (e.g. $4 \times 10^{11}$ Uranium ions per pulse) are two orders of magnitude higher than previously achieved at SIS18, posing extra challenges to the beam quality in SIS18 which in turn required upgrade of the current facility. The upgrade plan [59] included vacuum improvements to avoid beam losses due to residual gas pressures, decrease of the charge of the reference particle ( $\mathrm{U}^{73+}$ to intermediate charge state $\mathrm{U}^{28+}$ ) in order to avoid space charge effects at high intensities, new injection system for a safe inflection of the intermediate charge states at $11.4 \mathrm{MeV} / \mathrm{u}$, installation of the new $\mathrm{RF}$ cavity for the harmonic number $h=2$, upgrade of the main power supplies in order to reach higher cycling frequencies and longitudinal and transverse feedback systems. A schematic of the proposed FAIR facility is shown in Fig. 3.1 while the comparison between the parameters of SIS18 and SIS100 synchrotrons is given in Table 3.1. Closed orbit correction in SIS18 has also been planned as part of the upgradation of SIS18 in order to achieve higher beam intensities. The closed orbit correction in hadron synchrotrons is usually needed due to the two main reasons:

- In order to avoid particle losses because of the decrease in the transverse aperture due to the large closed orbit distortions. For SIS18, the beam size is of the order of 10 $\mathrm{mm}$ as shown in Fig. 3.2 for the horizontal plane which in this case is $\sigma_{x}=8 \mathrm{~mm}$ at injection. 


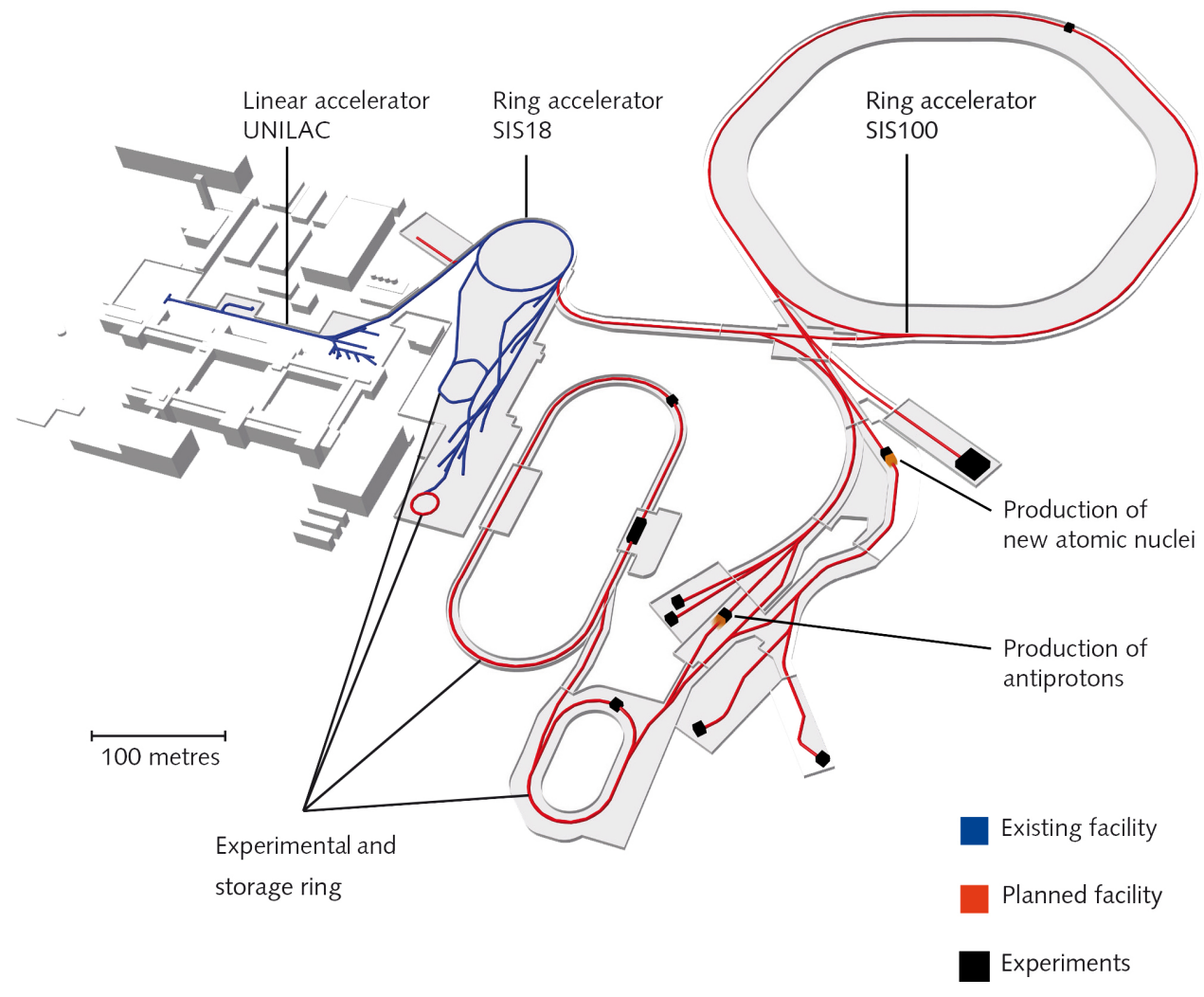

Figure 3.1 Layout of the upcoming FAIR facility [60. SIS18 is the booster ring of SIS100 synchrotron.

Table 3.1 Parameters of FAIR synchrotrons $58,61,62$.

\begin{tabular}{lll}
\hline \hline Parameter & SIS18 & SIS100 \\
\hline Circumference & $218 \mathrm{~m}$ & $1083.6 \mathrm{~m}$ \\
\hline Magnetic rigidity & $18 \mathrm{Tm}$ & $100 \mathrm{Tm}$ \\
\hline Injection energy & $11 \mathrm{MeV} / \mathrm{u}$ for $\mathrm{U}^{28+}$ & $200 \mathrm{MeV} / \mathrm{u}$ for $\mathrm{U}^{28+}$ \\
& $70 \mathrm{MeV} / \mathrm{u}$ for protons & $4.5 \mathrm{GeV} / \mathrm{u}$ for protons \\
\hline Extraction energy & $200 \mathrm{MeV} / \mathrm{u}$ for U ${ }^{28+}$ & $2.7 \mathrm{GeV} / \mathrm{u}$ for $\mathrm{U}^{28+}$ \\
& $4.5 \mathrm{GeV}$ for protons & $29 \mathrm{GeV}$ for protons \\
\hline Beam intensity & $1.5 \times 10^{11}$ ions & $6 \times 10^{11}$ ions \\
(per pulse) & $5 \times 10^{12}$ protons & $4 \times 2^{13}$ protons \\
\hline Magnets & Normal conducting & Super conducting \\
\hline Ramp rate & $10 \mathrm{~T} / \mathrm{s}$ (maximum) & $4 \mathrm{~T} / \mathrm{s}($ maximum $)$ \\
\hline Repetition frequency & $2.7 \mathrm{~Hz}$ & $0.7 \mathrm{~Hz}$ \\
\hline Beam size horizontal (RMS) & $10 \mathrm{~mm}$ at injection & $2-10 \mathrm{~mm}$ at injection \\
\hline
\end{tabular}




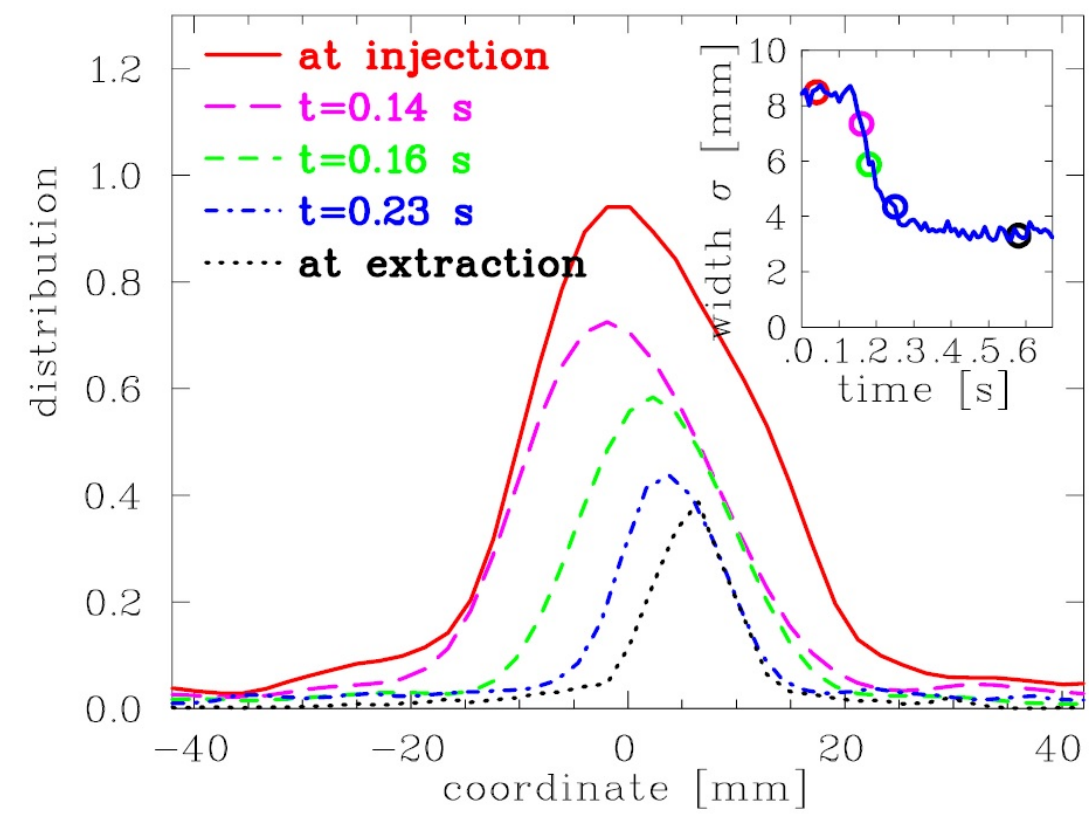

Figure 3.2 Measured transverse beam profile in horizontal plane for SIS18 over various times from injection to extraction.

Table 3.2 Parameters for the upgraded SIS18 as the booster ring of SIS100 16, 62.

\begin{tabular}{lll}
\hline \hline Parameter & SIS18 (old) & SIS18 (upgraded) \\
\hline Reference ion & $\mathrm{U}^{73+}$ & $\mathrm{U}^{28+}$ \\
\hline Maximum energy & $1 \mathrm{GeV} / \mathrm{u}$ & $0.2 \mathrm{GeV} / \mathrm{u}$ \\
\hline $\begin{array}{l}\text { Maximum intensity } \\
\text { (ions per pulse) }\end{array}$ & $4 \times 10^{9}$ ions & $1.5 \times 10^{11}$ ions \\
\hline Repetition frequency & $0.3-1 \mathrm{~Hz}$ & $2.7 \mathrm{~Hz}$ \\
\hline
\end{tabular}

- In order to keep the closed orbit within the linear field regions of the quadrupole and sextupole magnets as the nonlinear fields can deteriorate the beam quality (e.g. emittance and consequently the brightness) which cannot be retained for hadron beams even at higher energies due to the lack of the synchrotron damping.

\subsection{Peculiar behaviors of SIS18 synchrotron in the con- text of COFB system}

The dynamic orbit correction over the entire ramp in SIS18 imposes new challenges for the realization of the closed orbit feedback system in comparison to the state-of-the-art systems becuase of some unique features of the SIS18 synchrotron, as explained below. 

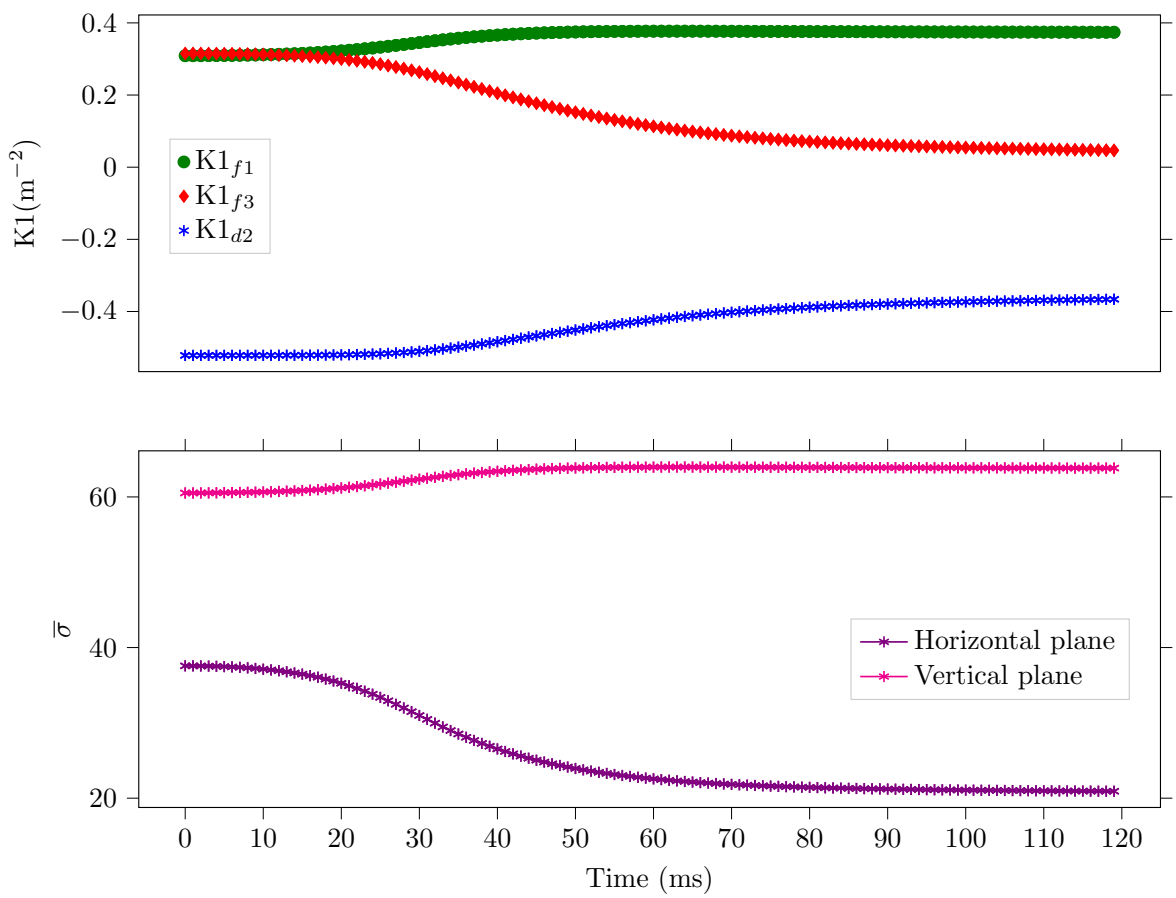

Figure 3.3 Top: Triplet to doublet quadrupole transition over the ramp of $10 \mathrm{~T} / \mathrm{s}$ in SIS18 (an example of optics change during acceleration cycle). $\mathrm{K} 1_{f 1}, \mathrm{~K} 1_{d 2}$ and $\mathrm{K} 1_{f 3}$ are the normalized strengths of first, second and third singlets quadrupole families of SIS18, respectively. Bottom: The variation of the highest singular values of the ORM in both transverse planes, over the ramp of $10 \mathrm{~T} / \mathrm{s}$.

\section{Triplet to doublet transition}

SIS18 is a very versatile synchrotron that can be used for a vast variety of ions from protons to uranium accelerated to various energies (maximum $1 \mathrm{GeV} / \mathrm{u}$ depending upon charge-to-mass ratio) in acceleration cycles of various lengths in times (100 ms to $1 \mathrm{~s}$ ). Each of the 12 machine periods contains two bending magnets and a triplet group of two long doublet quadrupoles and one additional triplet lens (Fig. 2.1). One of the peculiar behavior of SIS18 is the transition from triplet to doublet focusing from injection to extraction, meaning that triplet is reduced to doublet by switching off the third quadrupole singlet up to the extraction as shown in Fig. 3.3 (top) for a ramp of maximum dipole field gradient of $10 \mathrm{~T} / \mathrm{s}$, where the third singlet strength is marked as $\mathrm{K}_{f 3}$. The triplet focusing at injection leads to a fairly large transverse machine acceptance primarily in horizontal plane required for the multi-turn injection due to the low injection energy of $11.4 \mathrm{MeV} / \mathrm{u}$ and the relatively large beam emittance for heavy ions. On the other hand, at higher energies, the focusing is switched to doublet configuration which requires less quadrupole magnet currents and make beam extraction and chromatic control easier. The effect of quadrupole strengths is directly visible in the beta function pattern throughout the synchrotron. A comparison of the beta functions calculated with the help of MAD-X 63] corresponding to the quadrupole strengths for injection and extraction 

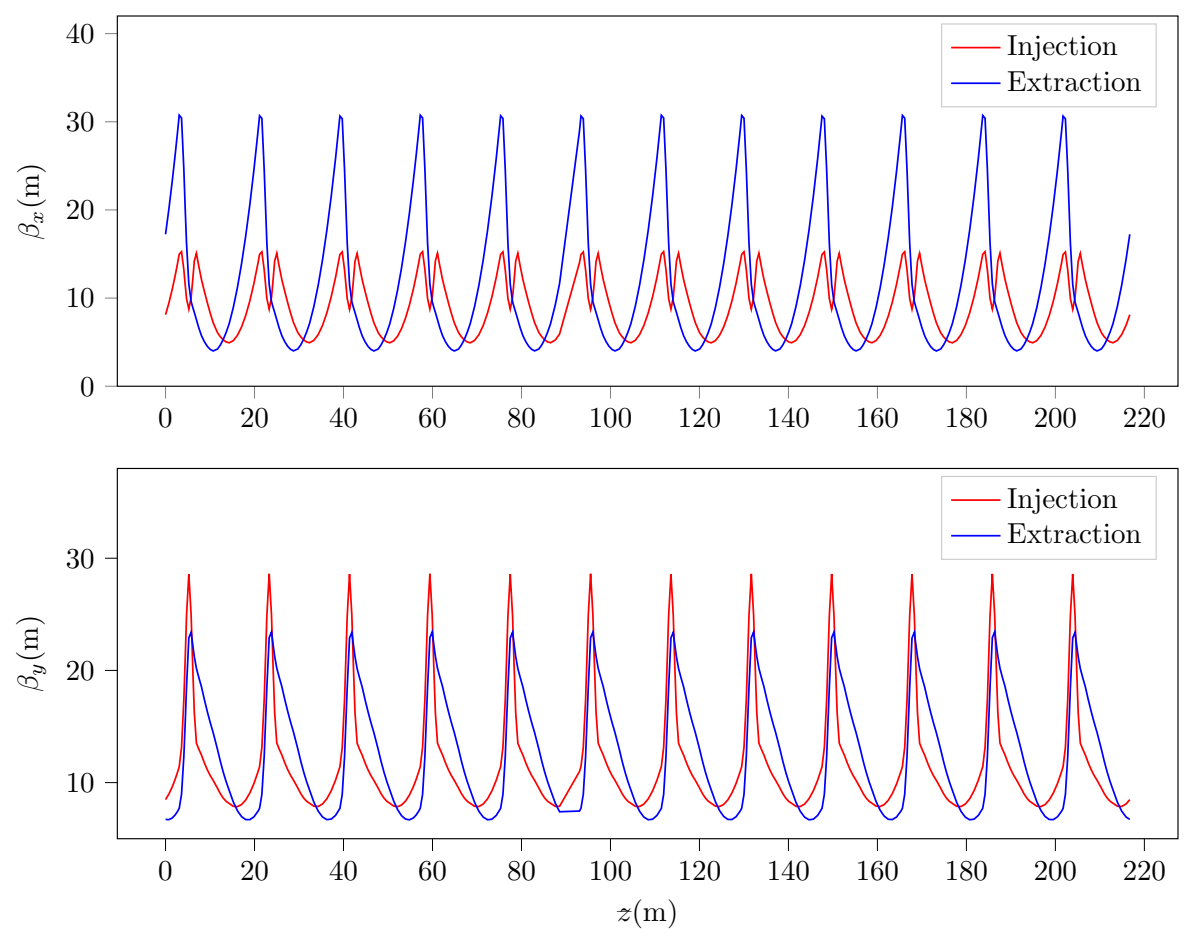

Figure 3.4 Beta function corresponding to the quadrupole strengths at injection and extraction related to the focusing strengths depicted in Fig. 3.3 for SIS18. Horizontal (top) and vertical (bottom).

is shown in Fig. 3.4. One can see that the maximum beta functions at injection energy is smaller than that corresponding to the extraction energy where the machine acceptance is defined as 64],

$$
A_{w}=\pi \min \left\{\frac{a_{w}^{2}(z)}{\beta_{w}(z)}\right\}
$$

where $A_{w}$ is the acceptance (mm mrad) in either transverse direction and $a_{w}$ is the minimum aperture radius. The transverse acceptance for SIS18 is larger at injection energy in the horizontal plane as multi-turn injection is done primarily in this plane.

The triplet to doublet transition causes a systematic change in the orbit response matrix of SIS18 over the ramp by varying the beta functions and phase advances at the locations of BPMs and correctors (according to Eq. 2.23), which is otherwise regarded as fixed model for the state-of-the-art COFB systems at other facilities. The highest singular values for the ORMs calculated in MAD-X using the quadrupole strength patters of Fig. 3.3 (top) are plotted in Fig. 3.3 (bottom) for both transverse planes. One can see a major change in the horizontal ORM over the ramp.

It is important to mention that the quadrupole strength variation over the ramp is intended to keep the horizontal and vertical tune values nearly constant but a tune shift over the ramp has also been measured experimentally adding another source of ORM variation. Figure 3.5 shows the on-ramp measured tune variation in both planes. As a typical value, tune shift of 


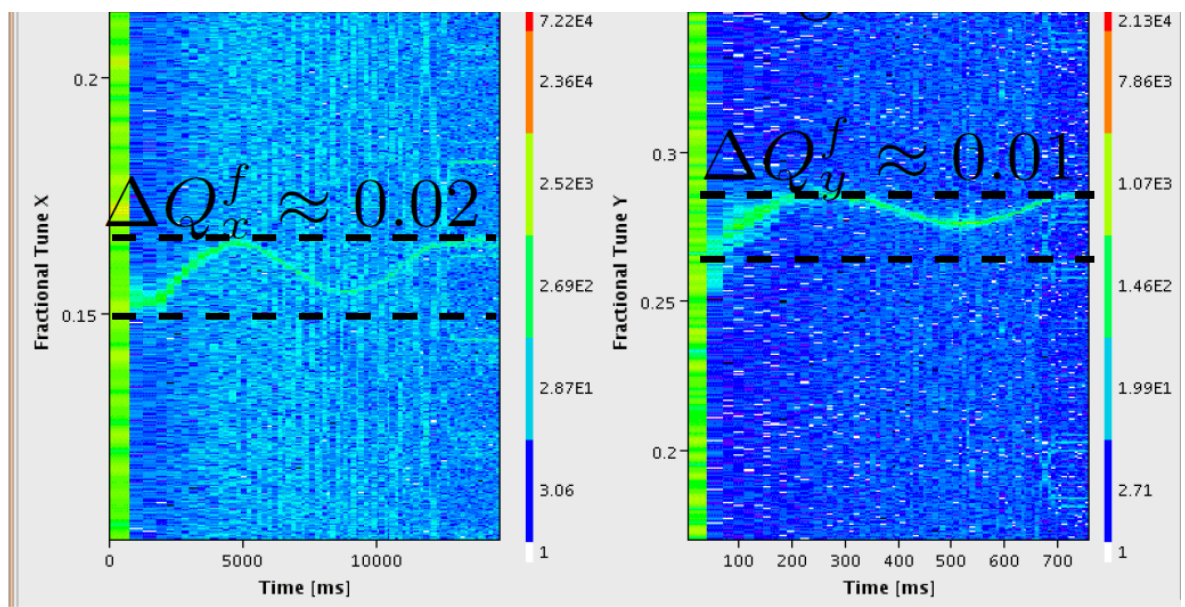

Figure 3.5 Measured tune variation over a typical ramp in SIS18 |53.

0.02 has been observed in horizontal plane.

\section{High frequency ripples}

The ripple in the output current of the power supply of a dipole magnet appear in its magnetic field and can be seen as fast changing dipole error by the closed orbit. The frequency of ripple coupled to the orbit motion depends upon the thickness of the vacuum chamber. In SIS18, the thickness of the vacuum chambers inside the dipole and quadrupole magnets is kept very small in order to avoid the eddy current effects in the walls of the chambers, during the fast ramps. Table 3.3 lists the dimensions for the SIS18 elliptical vacuum chambers. The vacuum chamber can be modelled as a low pass filter as discussed in sec. 2.8.3 where the cut-off frequency for the dipole chamber has been calculated to be $10 \mathrm{kHz}$. Such a high cut-off frequency is beneficial, on one hand to apply the fast changing dipole and quadrupole magnetic ramps to the beam, but on the other hand, let the high frequency power supply ripple to couple to the closed orbit motion. Figure 3.6 shows the frequency content of the beam motion during the full acceleration cycle at one BPM of SIS18. The $150 \mathrm{~Hz}$ mains frequency and its higher harmonics are clearly visible. Therefore, the COFB system capable of disturbance rejection as high as possible up to $1 \mathrm{kHz}$ is envisaged.

Table 3.3 Dimensions of the vacuum chambers for dipole and quadrupole magnets in SIS18 [51].

\begin{tabular}{|c|c|c|}
\hline Parameter & Dipole chamber & Quadrupole chamber \\
\hline Major axis radius & $190.7 \mathrm{~mm}$ & $200.36 \mathrm{~mm}$ \\
\hline Minor axis radius & $70.7 \mathrm{~mm}$ & $116.36 \mathrm{~mm}$ \\
\hline Wall thickness & $400 \mu \mathrm{m}$ & $300 \mu \mathrm{m}$ \\
\hline
\end{tabular}




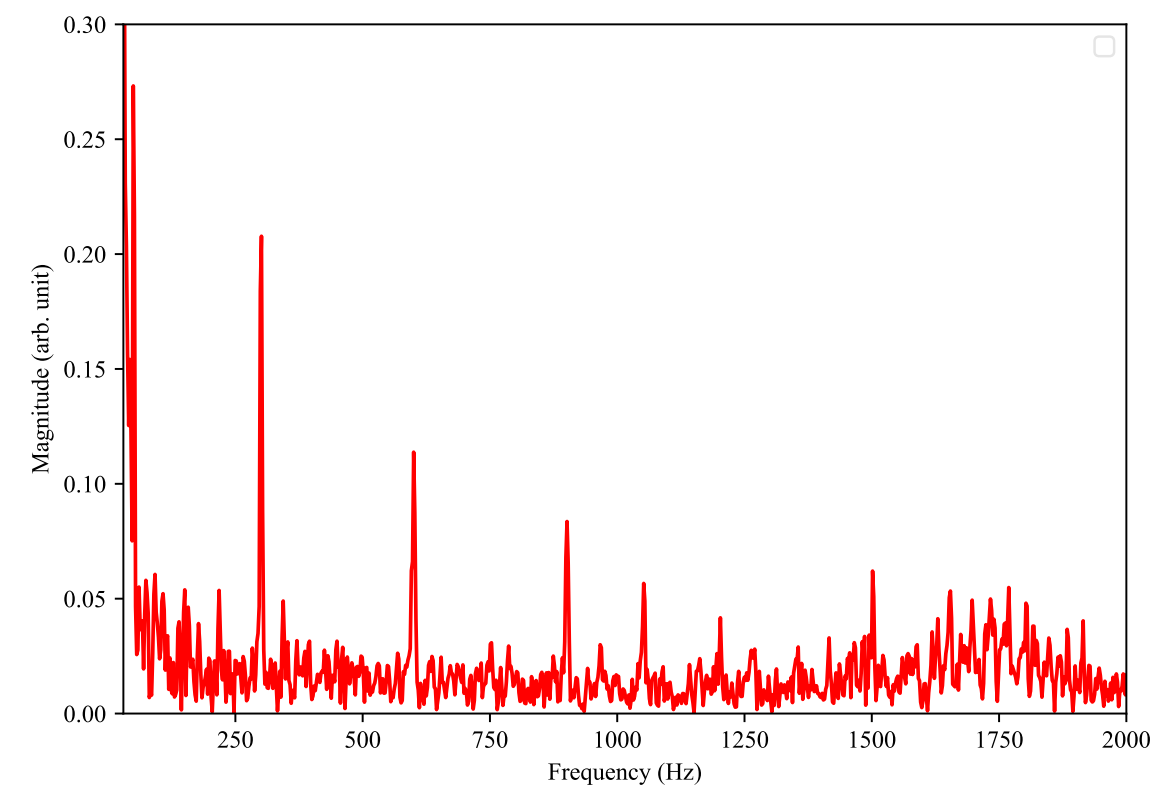

Figure 3.6 Mains frequency and its higher harmonics coupled to the closed orbit in SIS18.
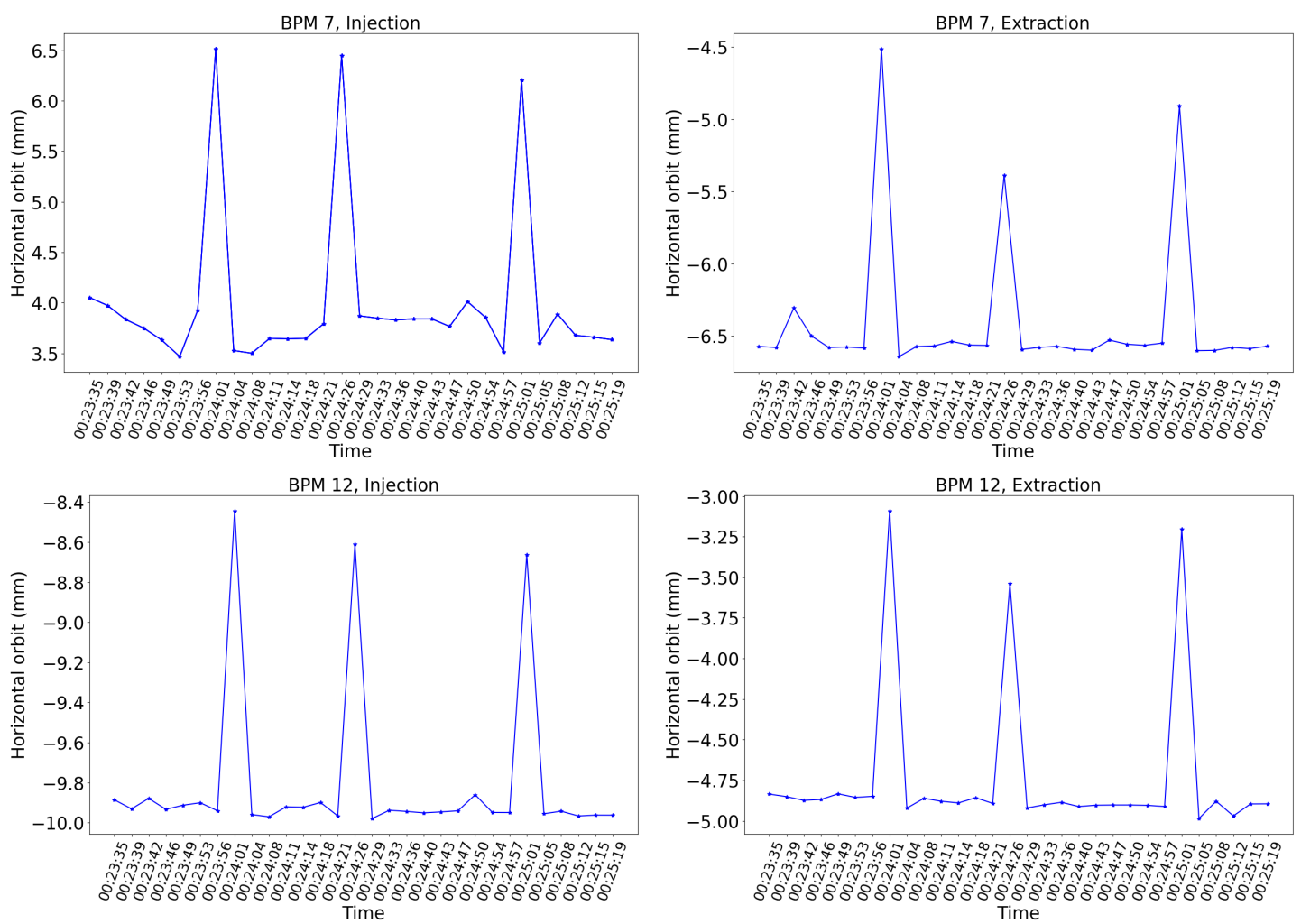

Figure 3.7 Measured beam position over 30 consecutive cycles at two BPM locations in SIS18, for injection and extraction settings. Measurement was made in July 2018. 


\section{Cycle-to-cycle influences}

Several users (up to 16) can be supported in "parallel" operation presently at SIS18 where the users can request dynamic changes in beam energy and intensity. This often leads to non-reproducible orbits due to magnet hysteresis which in turn depends strongly upon the mode of accelerator operation. In this case, pre-calculated correction strengths for the DC orbit shifts can also cause further orbit distortion due to sudden change in the perturbed orbit pattern and a feedback system is required even for slow/DC orbit shifts. Figure 3.7 show a measurement of sudden change in the DC beam position over many cycles.

\subsection{Demands for the SIS18 COFB system}

The unique features and versatile applications of SIS18 synchrotron require following parameters for the on-ramp COFB system.

- The COFB system should exhibit robustness against the ORM variation during the acceleration cycles of various time lengths and dipole field gradients.

- The possibility of delivering different ion beams to several users in alternate acceleration cycles in the same operation mode of SIS18 requires a COFB system robust against cycle-to-cycle hysteresis effects.

- High frequency ripples observed in Fig. 3.6 require a fast feedback system with disturbance rejection upto several hundred $\mathrm{Hz}$. A reasonably anticipated bandwidth is upto $600 \mathrm{~Hz}$.

- A stabilization of the closed orbit for a maximum RMS deviation below $1 \mathrm{~mm}$ is anticipated for a typical beam size $\sigma_{x}=10 \mathrm{~mm}$. This is in accordance with stability criteria of $10 \%$ of the beam size generally accepted in the light source community.

This kind of COFB system has not been realized before at any facility within the combination of these specifications. The goal of this Ph.D. thesis is to investigate the feasibility of a fast and robust $\mathrm{COFB}$ system in terms of the performance and stability for the on-ramp orbit correction in SIS18 and demonstrate its realization with beam-based measurements. 


\section{Chapter Four}

\section{Symmetry exploitation in the orbit response matrix}

In this chapter, a one dimensional discrete Fourier transform (DFT)-based diagonalization and inversion of the orbit response matrix (ORM) for symmetric lattices is presented, for the first time in the COFB system community. The technique is based upon the exploitation of circulant symmetry in the lattice and provides information compression into a diagonal matrix since the left and right orthogonal matrices are standard Fourier matrices. This method serves as the transition between previously discussed harmonic analysis and SVD with an exact equivalence for symmetric lattices. Further, a nearest-circulant extension is discussed for broken symmetries making most of the ideas discussed for symmetric matrices applicable to those of near-symmetric lattices.

\subsection{Orbit correction methods: a brief comparison}

The global orbit correction in synchrotrons is based upon using a finite number of correctors to modify the closed orbit at the locations of BPMs where the linear response of closed orbit at each BPM to each corrector is given by Eq. 2.23. The historical development and applications of the global orbit correction methods have been discussed in sec. 2.7 where it is mentioned that singular valued decomposition (SVD) of the ORM has become a de facto method for the orbit correction in synchrotrons. It is important to clarify that SVD method of closed orbit correction is in fact an ORM-based correction method and SVD is just a mathematical recipe to invert (pseudo-invert) the ORM. All the nice properties of SVD e.g. representation of the closed orbit in a mode space (Eq. 2.39) can be exploited only because of using the ORM for the calculation of correction values of the corrector magnets. Before discussing the merits and demerits of SVD, let us compare the two methods of global orbit correction, the sliding bump method and the ORM-based calculation of corrector-strengths for the vertical plane of SIS18 with the help of simulation using the MAD-X model of the synchrotron. A non-dynamical fixed perturbed orbit is generated with the help of random misalignment of all quadrupoles (in MAD-X) in a Gaussian distribution within the range of $\pm 0.5 \mathrm{~mm}$ and is plotted in Fig. 4.1 at the location of BPMs, in red. A straight line interpolation is made for the closed orbit between BPM locations for the purpose of visualization. In order to use the sliding bump method, the local bump condition using three correctors is given in Eq. 2.24 


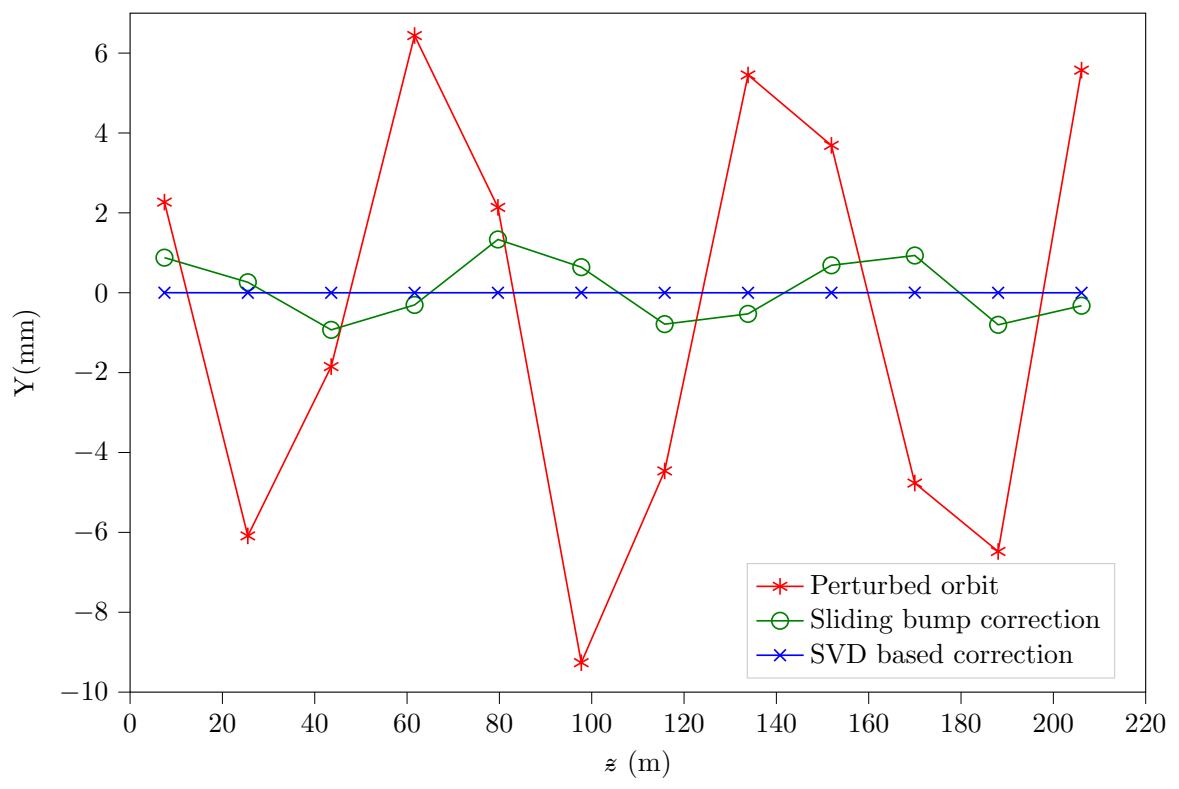

Figure 4.1 Comparison of sliding bump and SVD method for the correction of a static orbit correction in SIS18 (simulation in MAD-X).

and can be re-written for vertical plane of SIS18 as [39],

$$
\begin{aligned}
& \theta_{2}=-2 \theta_{1} \cos (\Delta \phi) \\
& \theta_{3}=\theta_{1}
\end{aligned}
$$

where $\theta_{1}$ is an arbitrary angle, $\Delta \phi=\frac{2 \pi Q_{y}}{N}$ is the phase advance between any two consecutive correctors which are placed periodically throughout the synchrotron and $N=12$ is the total number of correctors. For each BPM location, three consecutive correctors are chosen, one from the same cell of the BPM and two from the two neighboring cells and $\theta_{i}$ are calculated for each corrector as the main contributor of one local bump as a direct consequence of Eq. 2.25 as,

$$
\theta_{i}=-\frac{y_{i+1}}{\left(\frac{\partial y_{i+1}}{\partial \theta_{i}}\right)}
$$

where $\frac{\partial y_{i+1}}{\partial \theta_{i}}$ is estimated by varying $\theta_{1}$ and applying $\left(\theta_{1}, \theta_{2}, \theta_{3}\right)$ obtained from Eq. 4.1 . For the BPM position marked as $y_{2}$, the set of correctors consists of $\theta_{1}, \theta_{2}$ and $\theta_{3}$. It is important to understand that on one hand, three correctors are used to "act" on one BPM and on the other hand, each corrector is also shared by three bumps and hence by three BPMs. Each corrector acts as the "main" contributor for each bump and as the "constrained" contributor for closing other two bumps created by the two preceding correctors. This can be mathematically illustrated by writing the total corrector-strength $\theta^{\prime}$ for a steerer used in three consecutive bumps using Eq. 4.1 and 4.2 as,

$$
\theta_{i}^{\prime}=-\frac{y_{i+1}}{\left(\frac{\partial y_{i+1}}{\partial \theta_{i}}\right)}-2 \theta_{i-1} \cos (\Delta \phi)+\theta_{i-2}
$$


where the first term is to make the closed orbit minimum at the BPM location marked as $y_{i+1}$ and second and third terms are to contribute in the local bumps created by the correctors marked as $\theta_{i-1}$ and $\theta_{i-2}$. Equation 4.3 infers that sliding bump method has $(N-2)$ degrees of freedom in terms of linearly independent correctors, regardless of number of BPMs. An orbit correction simulated in this case is shown in Fig. 4.1 in green where a residual is left over due to the effectively 10 out of 12 correctors being used.

In case of using the full ORM (and consequently using SVD for matrix inversion) for the corrector-strength calculation, the blue curve is obtained as a result of orbit correction. In this case, the closed orbit at each BPM location is effected by all correctors as,

$$
y_{i}=\sum_{j=0}^{N-1}\left(r_{i, j}\right)\left(\theta_{j}\right)
$$

where $r_{i, j}$ are the elements of the $i^{\text {th }}$ row of the ORM R (Eq. 2.23) and $\theta_{j}$ are the calculated corrector-strength using SVD-based inversion of the ORM. The above example shows that ORM-based orbit correction has rightly taken over all other methods for the global orbit correction.

\subsection{Limitations of SVD-based orbit correction}

SVD is a powerful technique for the decomposition and pseudo-inversion of any matrix of arbitrary size and dimension and its merits for the ORM inversion are listed already in sec. 2.7.4. Here we talk about the weaknesses of SVD technique that arose primarily in the context of SIS18 COFB requirements. SVD decomposes the ORM into left and right orthogonal matrices whose columns $\mathbf{u}_{i}$ and $\mathbf{v}_{i}$ act as the modes for the perturbed orbit and the corrector-strength vectors, respectively. There is no physical interpretation of SVD modes available except that they form an orthonormal space i.e. SVD modes can be collection of any numbers who obey the restriction given as,

$$
<\mathbf{u}_{i}, \mathbf{u}_{j}>=\delta_{i j}
$$

By physical interpretation, it is meant that there is no information available between the discrete points of the columns of $\mathbf{U}$ and $\mathbf{V}$ matrices, for the interpolation. Fig 4.2 shows the columns $\mathbf{u}_{i}$ and $\mathbf{v}_{i}$ plotted for the SIS18 ORMs calculated in MAD-X corresponding to typical injection settings, in both planes. The data points are connected via straight lines only for the purpose of visualization. Moreover, all the three matrices are interconnected through a phase relation between columns of $\mathbf{U}$ and $\mathbf{V}$ matrices. SVD-based diagonlization has following limitations in the context of SIS18 COFB system:

- SVD resolves the closed orbit into a mode space that has no physical meaning in order to relate different beam phenomena and their variation over the ramp with the resolved components of the closed orbit. 

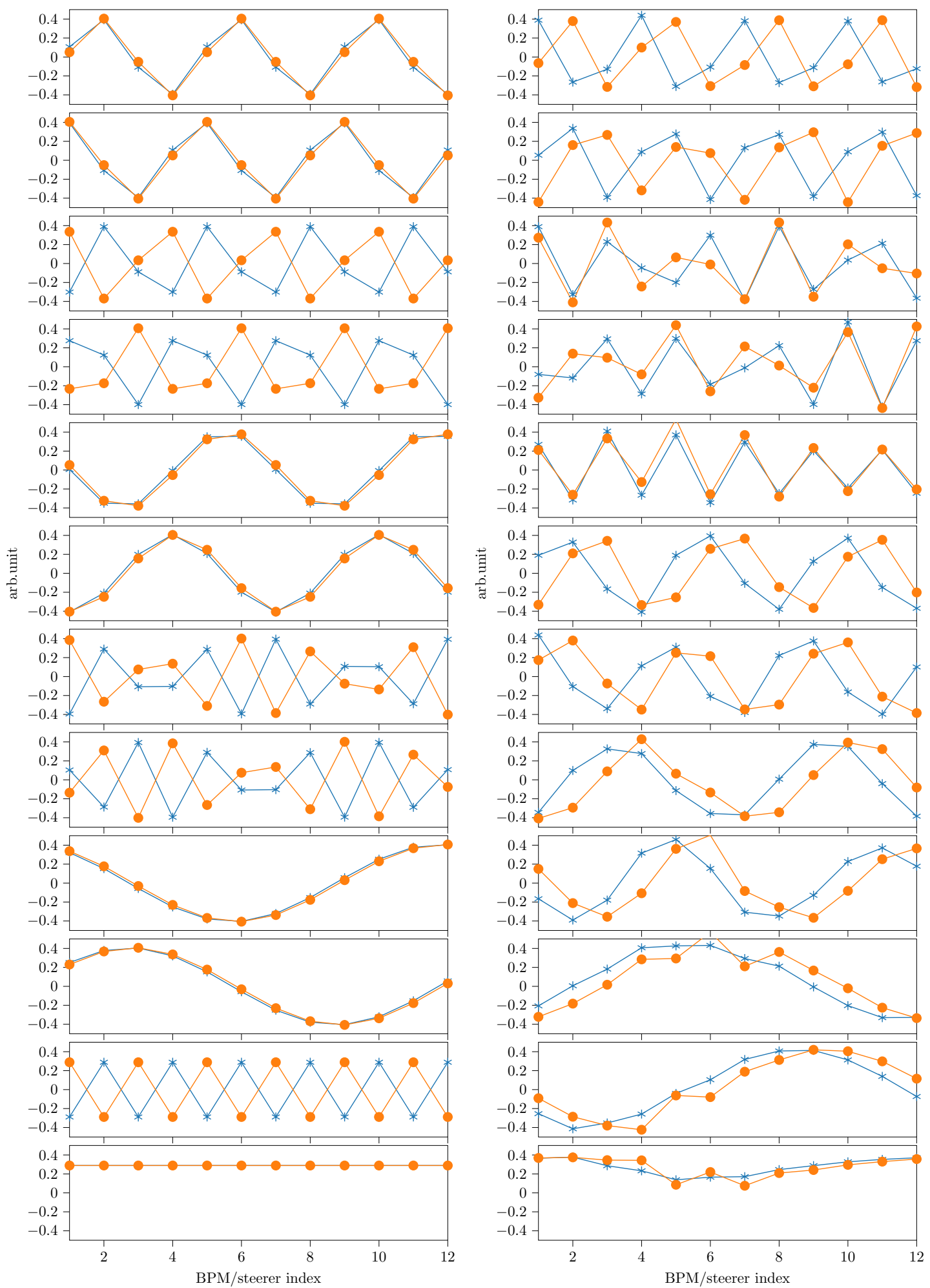

Figure 4.2 SVD modes (columns of $\mathbf{U}$ (blue) and V (orange) matrices). Left: vertical plane. Right: horizontal plane. 
- As discussed in sec. 3.2, the ORM changes from injection to extraction in both planes of SIS18 synchrotron because of the triplet to doublet transition. The lack of physical interpretation hinders to predict the evolution of SVD modes over the ramp. The interdependence between $\mathbf{U}, \mathbf{S}$ and $\mathbf{V}$ matrices requires the calculation and updating of all three matrices at each time step of the ORM update, during the ramp. Pre-calculation

and storage of the pseudo-inverses of the ORMs requires larger memory while online inversion needs a faster calculation in order to avoid delays.

- SVD also has some limitations particularly when dealing with uncertainty in the spatial process model as there is no apparent analytic way of associating uncertainties in the lattice parameters to the singular values [65]. Uncertainties appear into ORMs through various sources e.g. BPM and corrector calibration errors, tune variation due to magnet gradient errors or during the acceleration ramp. In case of SVD, the uncertainty $\Delta$ appear in all the three matrices of decomposition which e.g. in additive representation can be written as [3],

$$
\left(\mathbf{I}+\Delta_{R}\right) \mathbf{R}=\left(\mathbf{I}+\Delta_{U}\right) \mathbf{U}\left(\mathbf{I}+\Delta_{S}\right) \mathbf{S V}^{\mathrm{T}}\left(\mathbf{I}+\Delta_{V}\right)^{\mathrm{T}}
$$

- In case of a missing data point for example for the columns of $\mathbf{U}$ matrix, which corresponds to the scenario of ignoring a faulty beam position $w_{i}$, from heron called a "missing" BPM, a straight line interpolation will lose the information of that data point and one cannot predict the closed orbit position at the missing BPM location using SVD modes.

- Harmonic analysis was proposed as an alternative to SVD for the uncertainty modeling where the Fourier coefficients are found to be analytically related to the tune value $Q_{w}$ (Eq. 2.29] [3]. This formalism cannot be used for the matrix inversion and moreover, a quantitative relation between SVD singular values and Fourier coefficients was never found in literature, but is discussed in this thesis for the first time for the case of periodic arrangement of BPMs and correctors.

\subsection{Symmetry exploitation in the ORM}

Equation 2.23 represents the orbit response matrix and shows that it contains one global parameter, i.e. the betatron tune, while two local parameters, the beta function and phase advance, depend solely on the BPM and corrector locations. The global parameter determines the general criterion for the number of BPMs and correctors for an effective orbit correction. In order to ensure that modes up to twice the coherent betatron frequencies can be corrected, at least $4 \mathrm{BPMs}$ and correctors are required per betatron oscillation, ideally. The placement of BPMs and correctors is chosen at locations of higher beta function values in order to enhance the sensitivity of the closed orbit correction. However during the design of the synchrotrons, the symmetric arrangement of BPMs and correctors is usually neither given the importance it deserves nor exploited even if it exists. The only notable discussion of a symmetric layout for BPMs and correctors was found in [38] but was limited to finding eigenvalues for eigenvectorbased orbit correction. Any discussions towards the matrix inversion, relations to SVD and 


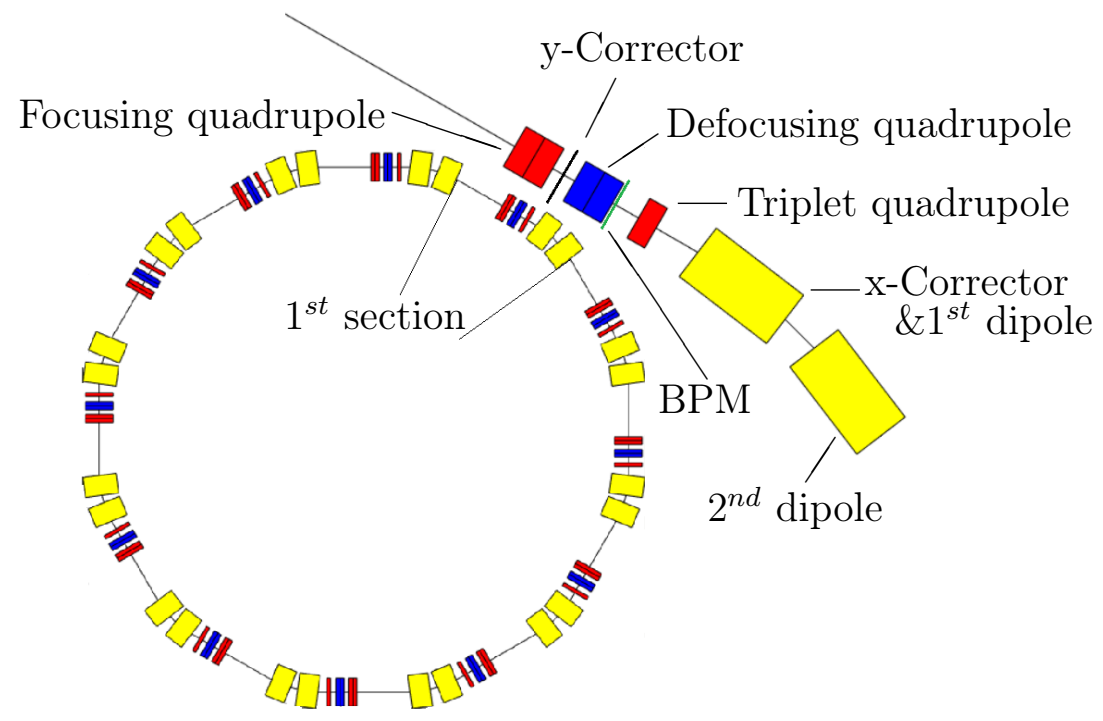

Figure 4.3 Schematic of the lattice of the SIS18 synchrotron. The first section has been magnified on top right and the components are labelled. The horizontal correctors are located at the $1^{\text {st }}$ dipole as extra windings in all sections except in the $4^{\text {th }}$ and $6^{\text {th }}$ sections where they reside on the second dipoles (not labeled in the figure).

application to broken symmetry were not made in this publication. These symmetry issues and their consequences will be addressed in this chapter for the first time in a stringent way. In the following section, we will discuss two kinds of ORM symmetries (so called circulant and block-circulant symmetries). Such an arrangement of BPMs and correctors is realized in two different synchrotrons of the FAIR project, the SIS18 and SIS100. They are used here as practical examples and to extend the findings to broken symmetries.

\subsubsection{Circulant symmetry of SIS18 vertical ORM}

The SIS18 synchrotron has a 12-fold symmetric lattice as shown schematically in Fig. 4.3. It comprises in the vertical plane of one BPM and one corrector each per section placed at the same location in each cell. In the horizontal plane, two correctors violate this symmetry which will be discussed later in the context of broken symmetry. The symmetric arrangement of BPMs and correctors in SIS18 vertical plane results in an equal phase advance $\Delta \phi$ between adjacent BPMs with same beta function for all BPMs. The correctors also have a similar arrangement such that,

$$
\begin{aligned}
\phi_{m+1 \bmod M} & =\phi_{m \bmod M}+\Delta \phi \\
\phi_{n+1 \bmod N} & =\phi_{n \bmod N}+\Delta \phi \\
\beta_{m+1, \bmod M} & =\beta_{m \bmod M} \\
\beta_{n+1 \bmod N} & =\beta_{n \bmod N}
\end{aligned}
$$


for $m=0, \ldots, M-1$ and for $n=0, \ldots, N-1$ where the modulo operator ensures the circular permutation of the synchrotron such that $\beta_{M}=\beta_{0}$ and $\phi_{M}=\phi_{0}$. Such an arrangement and same number of BPMs and correctors results into a special structure of the ORM called circulant matrix which is necessarily a square matrix and whose each row and column is a cyclic permutation of the previous row and column, respectively. The circulant symmetry in the elements of the ORM can be explained in terms of beta function and phase advance relations of Eq. 4.7 as,

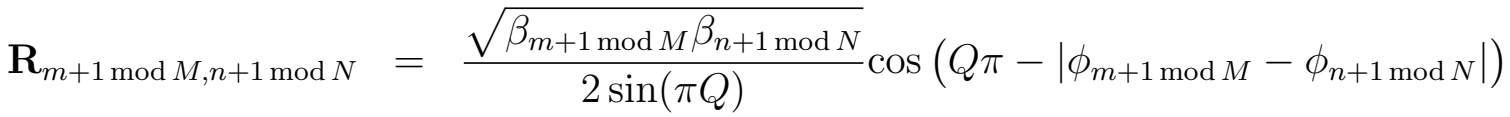

$$
\begin{aligned}
& =\frac{\sqrt{\beta_{m \bmod M} \beta_{n \bmod N}}}{2 \sin (\pi Q)} \cos \left(Q \pi-\left|\phi_{m \bmod M}+\Delta \phi-\phi_{n \bmod N}-\Delta \phi\right|\right)
\end{aligned}
$$

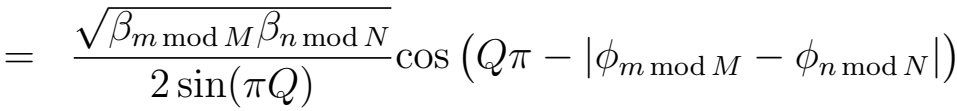

$$
\begin{aligned}
& =\mathbf{R}_{m \bmod M, n \bmod N}
\end{aligned}
$$

for $m=0, \ldots, M-1$ and for $n=0, \ldots, N-1$. A circulant matrix $\mathbf{R}_{C}$ of dimension $n$, can also be written as a sum of fundamental cyclic permutation matrices $\pi_{n}^{i}$, each of which is convoluted by the corresponding element $r_{i}$ of the first row or column of $\mathbf{R}_{C}$ as

$$
\mathbf{R}_{C}=r_{0} \pi_{n}^{0}+r_{1} \pi_{n}^{1}+\ldots \ldots . . r_{n-1} \pi_{n}^{n-1}=\sum_{i=0}^{n-1}\left(\pi_{n}^{i} r_{i}\right)
$$

where $i$ is the order of the permutation matrix. A general permutation matrix $\pi_{n}^{i}$ is a square matrix of size $n$ which can be generated as a result of the cyclic permutations of the identity matrix. The number of permutations $i(0 \leq i \leq n-1)$ is called the order of the permutation matrix. The identity matrix is a permutation matrix of order 0 . The set of fundamental permutation matrices for a dimension of 3 (as an example) are shown below:

$$
\pi_{3}^{0}=\left[\begin{array}{lll}
1 & 0 & 0 \\
0 & 1 & 0 \\
0 & 0 & 1
\end{array}\right], \pi_{3}^{1}=\left[\begin{array}{lll}
0 & 1 & 0 \\
0 & 0 & 1 \\
1 & 0 & 0
\end{array}\right], \pi_{3}^{2}=\left[\begin{array}{lll}
0 & 0 & 1 \\
1 & 0 & 0 \\
0 & 1 & 0
\end{array}\right] .
$$

The theory of circulant matrices is well established in literature 66 and they have very unique properties e.g. the whole circulant matrix can actually be represented by only one row or column. As an example, Eq. 4.11 shows the SIS18 vertical ORM calculated by MAD-X for triplet optics in the units of $\mathrm{mm} / \mathrm{mrad}$ :

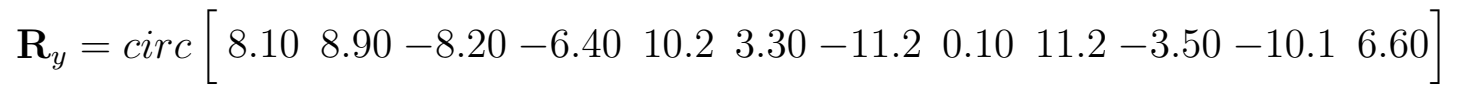

Here, the operator circ 66 is used to form a matrix by means of cyclic permutations of the row vector it contains as argument, whose elements are the $r_{i}$ of Eq. 4.9. 


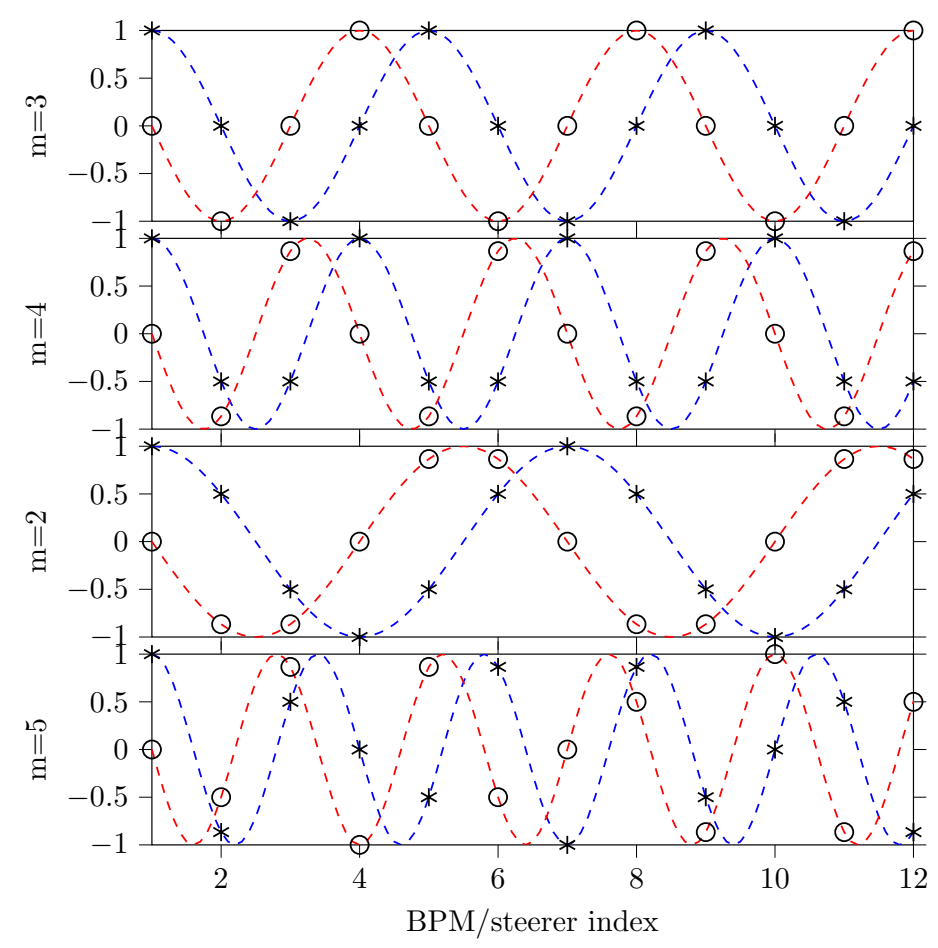

Figure 4.4 Fourier modes of order $m$. (Real (black dots) and imaginary (red dots) parts of the columns of Fourier matrix $\mathbf{F}$ ).

\subsubsection{Decomposition and inversion of the ORM}

A very useful property of circulant matrices, in the context of ORMs, is their diagonalization and decomposition by the discrete Fourier transform (DFT) of only one row or column, as explained below.

The DFT-based decomposition of a circulant matrix can be written as

$$
\mathbf{R}_{C}=\mathbf{F}^{*} \Lambda \mathbf{F}
$$

where $\mathbf{F}$ is a standard Fourier matrix which is identical for all circulants of same size with elements given as 67

$$
(\mathbf{F})_{f, i}=\frac{1}{\sqrt{n}} e^{j 2 \pi f i / n}=\frac{1}{\sqrt{n}}\left(\cos \left(\frac{2 \pi f i}{n}\right)+j \sin \left(\frac{2 \pi f i}{n}\right)\right)
$$

for $i, f \in[0, . ., n-1]$ where $i$ represents the sampling points, $f$ is the discrete frequency of each Fourier mode (column of $\mathbf{F}$ ), $j$ is the imaginary unit and $n$ is the size of the square circulant matrix. $\boldsymbol{\Lambda}$ is a diagonal matrix containing the discrete Fourier coefficients $v_{f}$ of the first row or column of $\mathbf{R}_{C}$ on its diagonal positions, which are given as,

$$
v_{f}=\operatorname{Re}\left\{v_{f}\right\}+j \operatorname{Im}\left\{v_{f}\right\}=\sum_{i=0}^{n-1} r_{i} e^{-j 2 \pi f i / n}
$$


The rows/columns of Fourier matrix and their corresponding Fourier coefficients (indexed as $k$ ) are either real or in complex conjugate pairs according to the relation [68]

$$
\begin{aligned}
(\mathbf{F})_{n+2-k} & =(\overline{\mathbf{F}})_{k} \\
v_{n+2-k} & =\bar{v}_{k}
\end{aligned}
$$

for $k=\{2, \ldots, n\}$. If $n$ is even, then $k_{1}$ and $k_{(n / 2)+1}$ are real and if $n$ is odd, then only $k_{1}$ is real. Here $k_{1}$ corresponds to frequency $f=0$.

In the case of an ORM, $n$ is the total number of BPMs or correctors while the columns of matrix $\mathbf{F}$ represent the mode space of BPM and correctors comprised of pure sine and cosine functions. In this way, a DFT-based decomposition gives a physical interpretation to the mode-space of an ORM and would be equivalent to the harmonic analysis. Fig. 4.4 shows the real and imaginary parts of the first four dominant Fourier modes where the discrete data points can be interpolated with analytic sine and cosine functions, contrary to SVD.

The inverse of $\mathbf{R}_{C}$ can be written as

$$
\mathbf{R}_{C}^{-1}=\mathbf{F}^{*} \Lambda^{-1} \mathbf{F}
$$

where $\boldsymbol{\Lambda}^{-1}$ is the diagonal matrix having inverses of Fourier coefficients at its diagonal positions.

\subsubsection{Equivalence of SVD and DFT for circulant symmetry}

For the general matrices, there is no analytic information available to interpolate the SVD modes between discrete elements of $\mathbf{U}$ and $\mathbf{V}$ matrices, as SVD chooses specific mode basis vectors in order to satisfy the orthogonality of the $\mathbf{U}$ and $\mathbf{V}$ matrices. In case of circulant matrices, there exists an equivalence between SVD- and DFT-based diagonalization of the matrices and hence, SVD modes can also be represented as sine or cosine functions. The above mentioned equivalence is worked out by introducing the discrete Hartley transform [69] matrix H. Following equation 4.12 and using the theorem 4.1 of $|70|$ which states that the SVD of a circulant matrix can be written as

$$
\mathbf{R}_{C}=(\mathbf{H}(\mathbf{F}) \operatorname{Re}\{\boldsymbol{\Sigma}\}-\mathbf{H}(\overline{\mathbf{F}}) \operatorname{Im}\{\boldsymbol{\Sigma}\})|\boldsymbol{\Lambda}| \mathbf{H}(\mathbf{F})
$$

where Hartley transformation is defined as,

$$
\begin{aligned}
\mathbf{H}(\mathbf{F}) & =\operatorname{Re}\{\mathbf{F}\}+\operatorname{Im}\{\mathbf{F}\} \\
\mathbf{H}(\overline{\mathbf{F}}) & =\operatorname{Re}\{\mathbf{F}\}-\operatorname{Im}\{\mathbf{F}\}
\end{aligned}
$$

The diagonal matrix $\boldsymbol{\Lambda}$ of the DFT-based decomposition can be written as,

$$
\Lambda=\Sigma|\Lambda|
$$

with $\boldsymbol{\Sigma}$ and $|\boldsymbol{\Lambda}|$ are the diagonal matrices containing the phases $\phi_{d i}$ and magnitudes of each Fourier coefficient, respectively. The matrices $\mathbf{U}, \mathbf{S}$ and $\mathbf{V}$ are calculated below by solving 
the right hand side of Eq. 4.17 element-wise. The last term can be solved as

$$
\begin{aligned}
(\mathbf{H}(\mathbf{F}))_{f, i} & =\frac{1}{\sqrt{n}}\left(\cos \left(\frac{2 \pi f i}{n}\right)+\sin \left(\frac{2 \pi f i}{n}\right)\right) \\
& =\sqrt{\frac{2}{n}}\left(\cos \left(\frac{2 \pi f i}{n}+\phi\right)\right) \\
& =\left(\mathbf{V}^{\mathrm{T}}\right)_{f, i}
\end{aligned}
$$

where $\phi=-\frac{\pi}{4}$. The singular values of the SVD matrix $\mathbf{S}$ are the moduli of the Fourier coefficients of DFT diagonal matrix $\Lambda$ :

$$
\mathbf{S}=|\Lambda|
$$

Similarly, the $\mathbf{U}$ matrix is equal to the following first part of the right hand side of Eq. 4.17 as,

$$
\begin{aligned}
& \left(\mathbf{H}(\mathbf{F}) \operatorname{Re}\{\boldsymbol{\Sigma}\}-\mathbf{H}(\overline{\mathbf{F}}) \operatorname{Im}\{\boldsymbol{\Sigma})_{f, i}\right. \\
& =\sqrt{\frac{1}{n}}\left(\cos \left(\frac{2 \pi f i}{n}\right)+\sin \left(\frac{2 \pi f i}{n}\right)\right) \cos \left(\phi_{d i}\right)-\frac{1}{\sqrt{n}}\left(\cos \left(\frac{2 \pi f i}{n}\right)-\sin \left(\frac{2 \pi f i}{n}\right)\right) \sin \left(\phi_{d i}\right) \\
& =\sqrt{\frac{1}{n}}\left(\cos \left(\frac{2 \pi f i}{n}-\phi_{d i}\right)+\sin \left(\frac{2 \pi f i}{n}-\phi_{d i}\right)\right) \\
& =\sqrt{\frac{2}{n}}\left(\cos \left(\frac{2 \pi f i}{n}-\phi_{d i}-\frac{\pi}{4}\right)\right) \\
& =(\mathbf{U})_{f, i}
\end{aligned}
$$

Combining Eqs. 4.20-4.22, Eq. 4.12 can be written as

$$
\mathbf{R}_{C}=\mathbf{U S V}^{\mathrm{T}}
$$

where $\mathbf{U}$ spans the space of $\mathbf{w}$ i.e. ( $\mathbf{x}$ or $\mathbf{y}$ ) and $\mathbf{V}$ spans the space of $\boldsymbol{\Theta}$. Eqs. 4.20 and 4.22 also explain the phase relationship between the columns of $\mathbf{U}$ and $\mathbf{V}$ matrices which can be seen in Fig. 4.2 (left) for the SVD modes of the SIS18 ORM in vertical plane, where a pure circulant symmetry exists. The phase difference comes from the phase angle of the corresponding Fourier matrices. This conversion shows a significant difference between the two techniques in terms of information spread. SVD distributes the information (magnitude and phase of the Fourier coefficients) in all three matrices while DFT-based decomposition compresses all the information into one diagonal matrix. SVD-like mode truncation is also possible here by removing the Fourier coefficients of absolute values below a certain threshold along with corresponding columns of $\mathbf{F}$ thus preserving the main benefit of SVD while adding the benefits of harmonic analysis for square circulant matrices. The method has direct application for the ORM in the vertical plane of SIS18. 


\subsubsection{Broken symmetry and nearest-circulant approximation}

In many practical scenarios, the circulant symmetry of the ORM can be broken due to the non-symmetric placement of BPMs and correctors, presence of insertion devices, beta beating etc. For example, two horizontal correctors in SIS18 are placed in the second dipoles of the $4^{\text {th }}$ and $6^{\text {th }}$ sections while all others are in the first dipoles, hence breaking the circulant symmetry in the corresponding columns highlighted with red color in the ORM below calculated by MAD-X for triplet optics in the units of $\mathrm{mm} / \mathrm{mrad}$ :

$$
\mathbf{R}_{x}=\left[\begin{array}{rrrrrrrrrrrr}
-2.78 & -4.86 & -1.24 & 2.89 & -6.89 & 3.82 & 4.02 & -7.36 & 5.27 & 0.70 & -6.16 & 7.07 \\
7.07 & -2.77 & -4.87 & 1.66 & 6.45 & -5.32 & 2.27 & 4.04 & -7.36 & 5.26 & 0.71 & -6.16 \\
-6.15 & 7.06 & -2.77 & -4.98 & -1.25 & 2.9 & -6.88 & 2.26 & 4.03 & -7.35 & 5.26 & 0.71 \\
0.70 & -6.15 & 7.08 & -4.08 & -4.87 & 1.65 & 6.44 & -6.89 & 2.27 & 4.02 & -7.36 & 5.27 \\
5.26 & 0.71 & -6.16 & 5.26 & -2.77 & -4.99 & -1.25 & 6.45 & -6.89 & 2.26 & 4.04 & -7.36 \\
-7.35 & 5.26 & 0.71 & -2.56 & 7.07 & -4.08 & -4.86 & -1.25 & 6.44 & -6.88 & 2.26 & 4.03 \\
4.02 & -7.36 & 5.27 & -2.03 & -6.16 & 5.26 & -2.78 & -4.87 & -1.24 & 6.44 & -6.89 & 2.27 \\
2.26 & 4.03 & -7.36 & 5.12 & 0.71 & -2.57 & 7.07 & -2.77 & -4.87 & -1.25 & 6.45 & -6.90 \\
-6.89 & 2.26 & 4.03 & -4.44 & 5.26 & -2.02 & -6.15 & 7.07 & -2.78 & -4.86 & -1.25 & 6.44 \\
6.44 & -6.89 & 2.27 & 0.49 & -7.36 & 5.12 & 0.70 & -6.16 & 7.08 & -2.78 & -4.87 & -1.24 \\
-1.24 & 6.44 & -6.9 & 3.82 & 4.04 & -4.45 & 5.26 & 0.71 & -6.16 & 7.07 & -2.77 & -4.87 \\
-4.86 & -1.25 & 6.44 & -5.31 & 2.26 & 0.50 & -7.35 & 5.26 & 0.71 & -6.15 & 7.06 & -2.77
\end{array}\right]
$$

Once the symmetry is broken, an ORM cannot be represented anymore with its single row/column and the DFT cannot be used directly for the decomposition and inversion of the ORM. In order to make the DFT-based method applicable to practical scenarios, a slight modification of the ORM in order to find a nearest-circulant approximation is proposed as an alternate for broken symmetries. This is based upon the fact an iterative correction implemented for most orbit correction systems can still converge with a modified process model at the cost of more iterations or correction speed. Another example of ORM approximation found in literature is the Tikhonov regularization [71 of the ORM which is used to "weaken" the control action for higher order SVD modes corresponding to smaller singular values by a replacement represented by the symbol " $\rightarrow$ " below:

$$
\frac{1}{\sigma_{i}} \rightarrow \frac{\sigma_{i}}{\sigma_{i}^{2}+\mu}
$$

Here the $\sigma_{i}$ are the singular values and an appropriate value of $\mu>0$ serves to filter out the effect of singular values for which $\sigma_{i}^{2} \ll \mu$, hence effectively decreasing the condition number of the ORM [72]. Such an ORM approximation has been implemented in the COFB system of Diamond Light Source, UK [3].

The theory of nearest-circulant approximation is discussed in detail in 73 but has never been explored for the ORM inversion before. For a given square matrix $\mathbf{R}$, its nearest-circulant approximation $\mathbf{R}_{N C}=\operatorname{circ}\left[c_{0}, c_{1}, \ldots c_{n-1}\right]$ can be found by the Frobenius inner product [74] of $\mathbf{R}$ with permutation matrices $\pi_{n}^{i}$ as,

$$
c_{i}=\frac{1}{n}<\mathbf{R}, \pi_{n}^{i}>
$$

where $n$ is the size of both $\mathbf{R}$ and $\pi$ matrices and the order of the permutation matrix is $i=0, \ldots, n-1$. Frobenius inner product is a binary operation that takes two matrices of equal size as input and returns a number as a result of adding the element-wise inner products. For two real $m \times n$ matrices $\mathbf{A}$ and $\mathbf{B}$ it is defined as

$$
<\mathbf{A}, \mathbf{B}>=\sum_{i=1}^{m} \sum_{k=1}^{n} a_{i k} b_{i k} .
$$



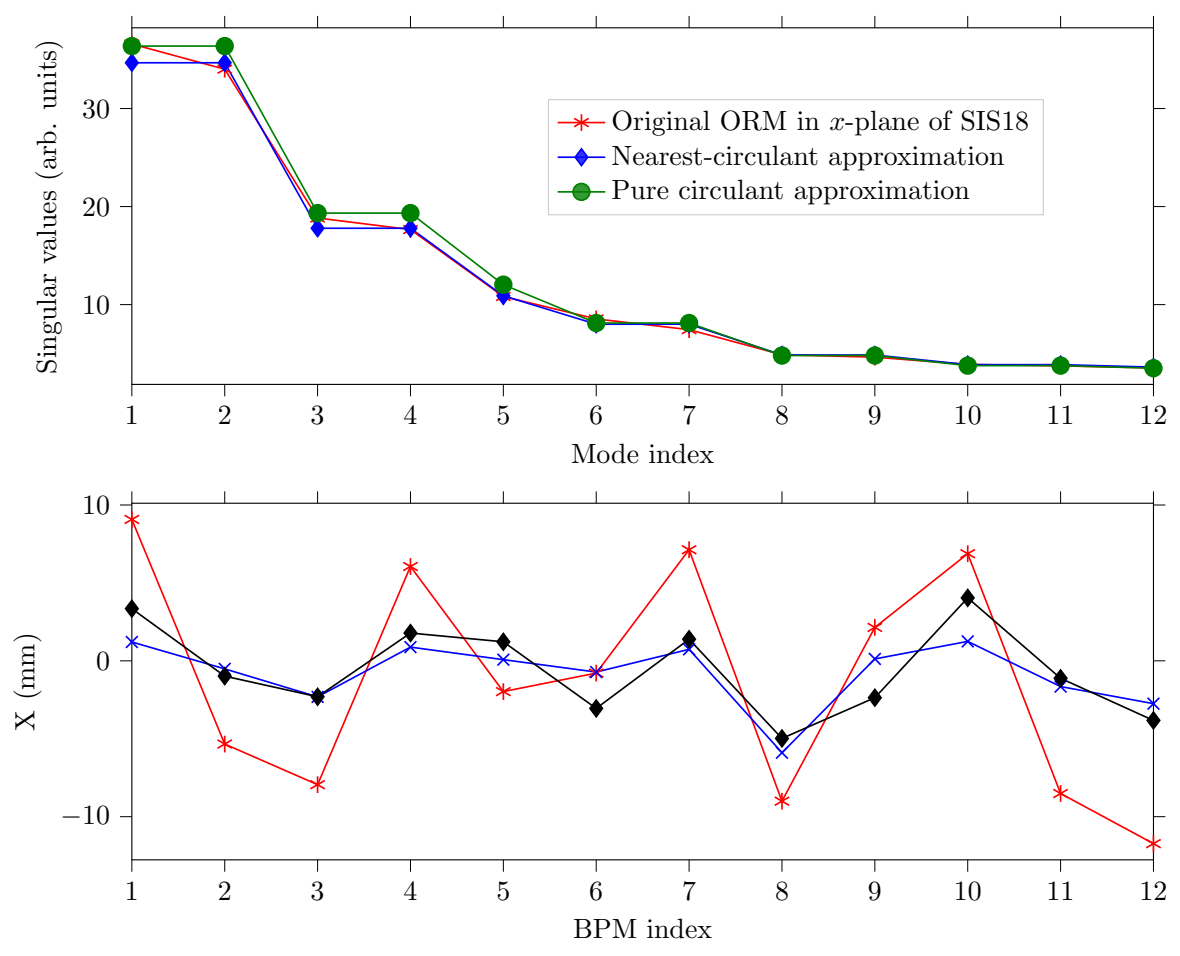

Figure 4.5 Top: Comparison of singular values of SIS18 ORM with its nearestcirculant approximation and its pure-circulant approximation in the $x$-plane. Bottom: Measured closed orbits in the $x$-plane of SIS18. Red('*'): Perturbed orbit $(\mathrm{RMS}=7.12 \mathrm{~mm})$. Blue(' $\mathrm{x}$ '): Corrected orbit using original ORM $(\mathrm{RMS}=2.15 \mathrm{~mm})$. $\operatorname{Black}\left({ }^{\prime} \diamond\right)$ : Corrected orbit using nearest-circulant approximation (RMS=2.82 mm).

Eq. 4.26 is equivalent to the averaging of those diagonal elements of $\mathbf{R}$ for which the corresponding entries in permutation matrix are non-zero. For the resultant approximation, the theory discussed in sections 4.3.1 4.3.3. holds.

\section{Experimental demonstration of using nearest-circulant approximation}

As mentioned before, the non-symmetric placement of two correctors breaks the pure circulant symmetry in the horizontal ORM of SIS18 and the nearest-circulant approximation is exploited in this case. Figure 4.5 (top) shows the calculated singular values of the SIS18 horizontal ORM and its nearest-circulant approximation for a qualitative comparison at typical settings corresponding to injection energy. Another scenario of replacing the bold columns in Eq. 2.24 with those that one would expect for a circulant matrix, called pure circulant approximation, is also considered for comparison. The mathematical treatment of using approximated ORM in the controller of COFB system is presented in the next chapter but the experimental demonstration of using such an approximation for the orbit correction is shown in Fig. 4.5 (bottom) where the red curve shows the measured perturbed closed orbit in the horizontal plane of SIS18. The perturbed orbit is measured in one acceleration cycle at injection by 
averaging over a time window of $40 \mathrm{~ms}$ (typical length of constant energy before start of ramp), while the orbit correction is performed in the next cycle with same machine settings. The corrector-strengths are calculated using the original ORM and its nearest-circulant approximation and applied one by one in two different cycles. The corrected orbits are shown in blue and black curves for the two cases, respectively. The difference in the RMS of the residual orbit is only $\simeq 9 \%$ of the initial distortion for both ORMs showing the usability of nearest-circulant approximation for orbit correction in SIS18.

\subsubsection{Block-circulant symmetry in the ORM}

SIS18 is a relatively small synchrotron in terms of number of BPMs and correctors i.e. 1 per section each, resulting into a possibility of pure circulant symmetry in the vertical plane and nearest-circulant approximation in the horizontal plane. In bigger synchrotrons, where more than one BPMs and correctors are placed in each cell, the phase advance between all adjacent BPMs and correctors is no more constant, resulting into the violation of the circulant symmetry. However, the repetition of the cell structure throughout the synchrotron and identical placement of BPMs and correctors in each cell may result in another kind of symmetry called block-circulant symmetry [66. If $p$ and $q$ are the number of BPMs and correctors in each cell placed identically in all cells and $k$ is the number of cells, the ORM attains a structure such that identical blocks of elements appear at the diagonal locations and the ORM can be expressed as,

$$
\mathbf{R}_{B C}=\operatorname{circ}\left[\mathbf{A}_{1}, \mathbf{A}_{2}, \mathbf{A}_{3} \ldots \ldots \mathbf{A}_{k}\right]
$$

Here $\mathbf{A}_{i}$ are the arbitrary matrices (not necessarily square) of dimension $p \times q$ and $\mathbf{R}_{B C}$ is a matrix of dimensions $k p \times k q$. The block-circulant symmetry in the elements of the ORM can also be expressed in terms of beta function and phase advance in analogy to Eq. 4.7 and 4.8 as,

$$
\begin{aligned}
\phi_{m+p \bmod M} & =\phi_{m \bmod M}+\Delta \phi \\
\phi_{n+q \bmod N} & =\phi_{n \bmod N}+\Delta \phi \\
\beta_{m+p \bmod M} & =\beta_{m \bmod M} \\
\beta_{n+q \bmod N} & =\beta_{n \bmod N}
\end{aligned}
$$

where $M$ and $N$ are the total number of BPMs and correctors. The elements of ORM repeat according to the following relation,

$$
\mathbf{R}_{m+p \bmod M, n+q \bmod N}=\mathbf{R}_{m \bmod M, n \bmod N}
$$

It is worth mentioning that despite of the presence of the insertion devices, the superperiodicity of the storage rings of many light sources can also be used to exploit the blockcirculant symmetry in the ORM. Few examples include ALBA of Spain [75], Sirius of Brazilian Light Source [76 and NSLS2 of Brookhaven National Laboratory [77.

A block-circulant matrix can also be written with the help of fundamental permutation 
matrices (Eq. 4.10) by replacing the numbers with matrices $\mathbf{A}_{i}$ in Eq. 4.9 66] (Theorem 56.4) as,

$$
\mathbf{R}_{B C}=\sum_{i=0}^{k-1}\left(\pi_{k}^{i} \otimes \mathbf{A}_{i+1}\right)
$$

where the symbol $\otimes$ denotes the Kronecker product [66] of the matrices. Given a $k \times k$ matrix $\mathbf{A}$ and $p \times q$ matrix $\mathbf{B}$, the Kronecker product $\mathbf{C}=\mathbf{A} \otimes \mathbf{B}$ is a matrix of size $k p \times k q$ given as

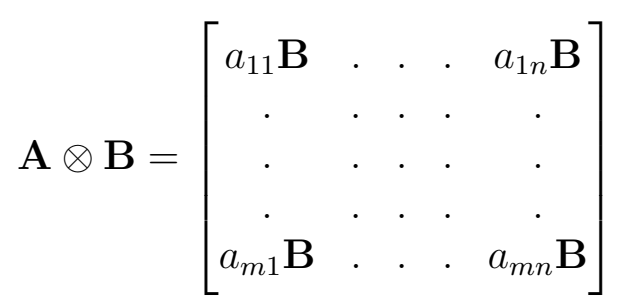

For arbitrary $\mathbf{A}_{i}$ in Eq. 4.31,

$$
\pi_{k}^{i} \otimes \mathbf{A}_{i+1}=\left(\mathbf{F}_{k}^{*} \Omega^{i} \mathbf{F}_{k}\right) \otimes \mathbf{F}_{p}^{*}\left(\mathbf{F}_{p} \mathbf{A}_{i+1} \mathbf{F}_{q}^{*}\right) \mathbf{F}_{q}
$$

where $\Omega$ is the diagonal matrix containing the Fourier coefficients of the permutation matrix. Let $\mathbf{B}_{i+1}=\mathbf{F}_{p} \mathbf{A}_{i+1} \mathbf{F}_{q}^{*}$, the Eq. 4.31 becomes

$$
\mathbf{R}_{B C}=\left(\mathbf{F}_{k} \otimes \mathbf{F}_{p}\right)^{*}\left(\sum_{i=0}^{k-1} \Omega^{i} \otimes \mathbf{B}_{i+1}\right)\left(\mathbf{F}_{k} \otimes \mathbf{F}_{q}\right)
$$

The middle term in Eq. 4.34 has a form of a diagonal matrix as

$$
\sum_{i=0}^{k-1} \Omega^{i} \otimes \mathbf{B}_{i+1}=\operatorname{diag}\left(\mathbf{M}_{1}, \mathbf{M}_{2}, \ldots, \mathbf{M}_{k}\right)=\mathbf{D}
$$

where $\mathbf{M}_{i}$ can be calculated by the relation

$$
\left[\begin{array}{c}
\mathbf{M}_{1} \\
\mathbf{M}_{2} \\
\cdot \\
\cdot \\
\mathbf{M}_{k}
\end{array}\right]=\left(m^{\frac{1}{2}} \mathbf{F}_{k}^{*} \otimes \mathbf{I}_{p q}\right)\left[\begin{array}{c}
\mathbf{B}_{1} \\
\mathbf{B}_{2} \\
\cdot \\
\cdot \\
\mathbf{B}_{k}
\end{array}\right]
$$

and Eq. 4.33 yields the following diagonalization of the block-circulant matrix,

$$
\mathbf{R}_{B C}=\left(\mathbf{F}_{k} \otimes \mathbf{F}_{p}\right)^{*}(\mathbf{D})\left(\mathbf{F}_{k} \otimes \mathbf{F}_{q}\right)
$$

where

$$
\mathbf{D}=\left[\begin{array}{cccccc}
\mathbf{M}_{1} & 0 & 0 & . & . & 0 \\
0 & \mathbf{M}_{2} & 0 & . & . & 0 \\
\cdot & . & \cdot & . & . & 0 \\
\cdot & . & . & . & . & 0 \\
0 & 0 & 0 & . & . & \mathbf{M}_{k}
\end{array}\right]
$$


$\mathbf{F}_{m}$ and $\mathbf{F}_{n}$ are the standard Fourier matrices defined in Eq. $4.13 . \mathbf{M}_{i}$ are the matrices of dimension $p \times q$ which contain all the information of the block-circulant matrix and can be calculated using only the first row of blocks. Equation 4.37 can be solved to calculate the inverse or pseudo-inverse $\left(\mathbf{R}_{B C}^{+}\right)$of the ORM as

$$
\mathbf{R}_{B C}^{+}=\left(\mathbf{F}_{k} \otimes \mathbf{F}_{p}\right)^{*}\left(\mathbf{D}^{+}\right)\left(\mathbf{F}_{k} \otimes \mathbf{F}_{q}\right)
$$

where

$$
\mathbf{D}^{+}=\operatorname{diag}\left(\mathbf{M}_{1}^{+}, \mathbf{M}_{2}^{+}, \ldots, \mathbf{M}_{k}^{+}\right)
$$

The nearest-block-circulant symmetry can also be exploited in matrices which deviate slightly from the pure block-circulant symmetry by averaging over the diagonal blocks instead of elements, in analogy to Eq. 4.26.

\subsubsection{SIS100 ORMs and symmetry exploitation}

The SIS100 which is the largest synchrotron of the FAIR project has a super-periodicity in its lattice with a six-fold symmetry of the cells where each section has 14 BPMs and 14 correctors. Anyhow, the pure block-circulant symmetry of its ORM is slightly broken due to the replacement of cryogenic quadrupole with the normal warm quadrupole in one section [58]. The warm quadrupole results in a beta beating (peak-peak $\approx 10 \%$ ) and hence a loss of symmetry in the ORM as calculated by MAD-X. The beta function at BPM locations in the vertical plane has been plotted in Fig. 4.6 for three consecutive sections with and without beta beating (by replacing the warm quadrupole by a cold quadrupole in MAD-X). One can see that the beta function repeats after every 14 data points i.e. number of BPMs in each section. The block symmetry of the SIS100 ORMs can be explored in two ways; either by ignoring beta beating or by finding the nearest-block-circulant approximation by averaging the diagonal blocks. For the SIS100 ORM, $k$ is the number of sections while $p$ is the number of BPMs or correctors in each section, hence the ORM can be approximated with a square block-circulant matrix, resulting from the equal number of BPMs and correctors in each section. The block diagonal matrices (D) of SIS100 ORMs for both the planes in case of ignoring the beta beating are visualized in Fig. 4.7 and 4.8 as Lego plots of their absolute values, respectively. It can be seen that very few elements have larger magnitude providing the liberty to remove weak modes before inversion just like singular values in SVD. Also, half of the block diagonal matrices have unique values in analogy to Eq. 4.15 as the diagonal blocks also appear in the complex conjugate pairs [68]. The rows of the block diagonal matrices containing the largest absolute values correspond to the tune modes of the Fourier matrices $\left(\mathbf{F}_{k} \otimes \mathbf{F}_{p}\right)$ which are plotted in Fig. 4.9 along with tune modes of SVD for horizontal plane. The equal integer part of the tune in both planes explains the same location of largest absolute values in both Lego plots. Moreover, a comparison of the singular values with the moduli of the row vectors of the block diagonal matrices in Fig. 4.10 provides a clear hint on the correspondence between the two methods.

Although the main focus of the thesis is the SIS18 COFB system, the symmetry exploitation in SIS100 ORMs is discussed for the completion of the topic and presenting the DFT-based diagonalization as an alternate to SVD for the majority of synchrotrons. 


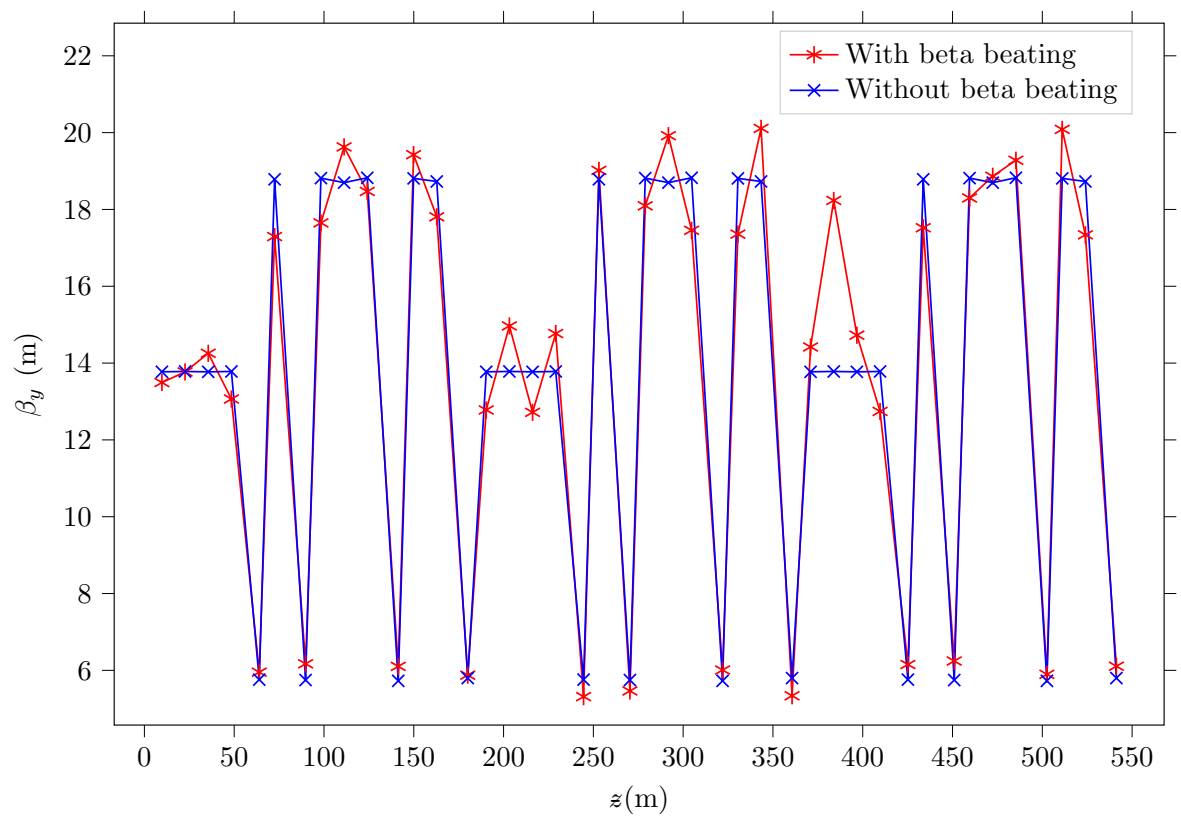

Figure 4.6 Beta function at BPM locations in the y-plane of SIS100 (simulated in MAD-X) plotted for three cells, with beta beating $\left(\operatorname{red}\left({ }^{*}\right.\right.$ ') $)$ and without beta beating (blue (' $x$ ')). The abscissa is the distance along the synchrotron.

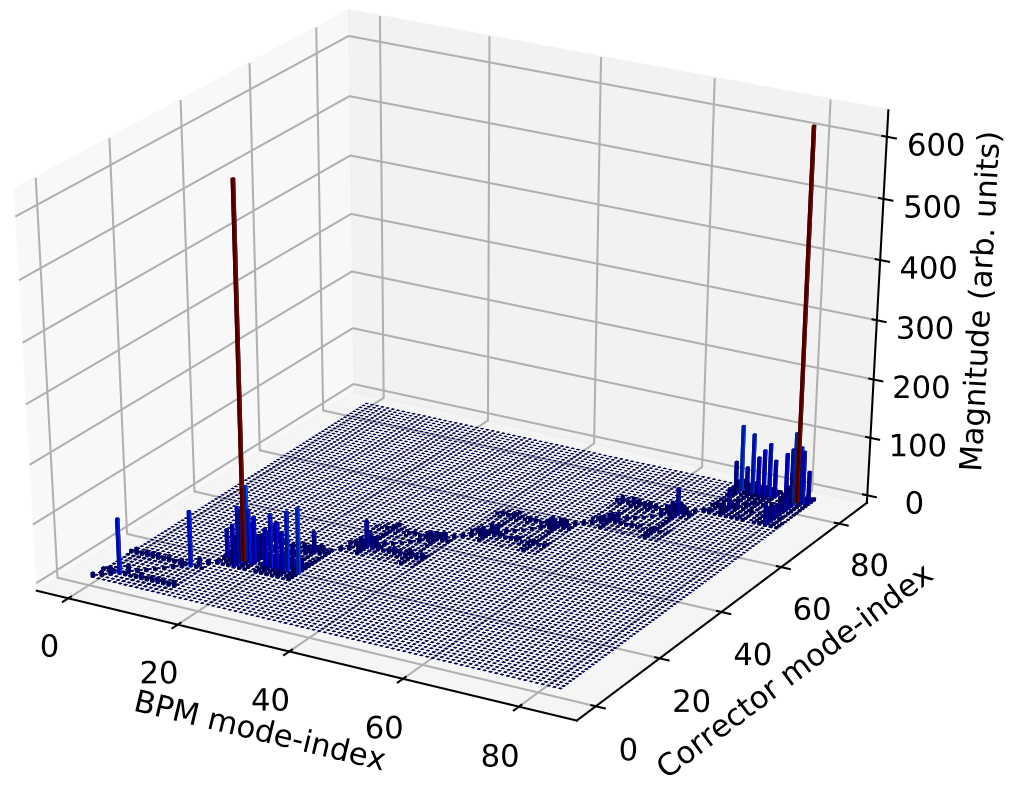

Figure 4.7 Magnitudes of the complex entries of the block diagonal matrix D defined in Eq. 4.38, calculated for the case of ignoring the beta beating in the $x$-plane of SIS100. 


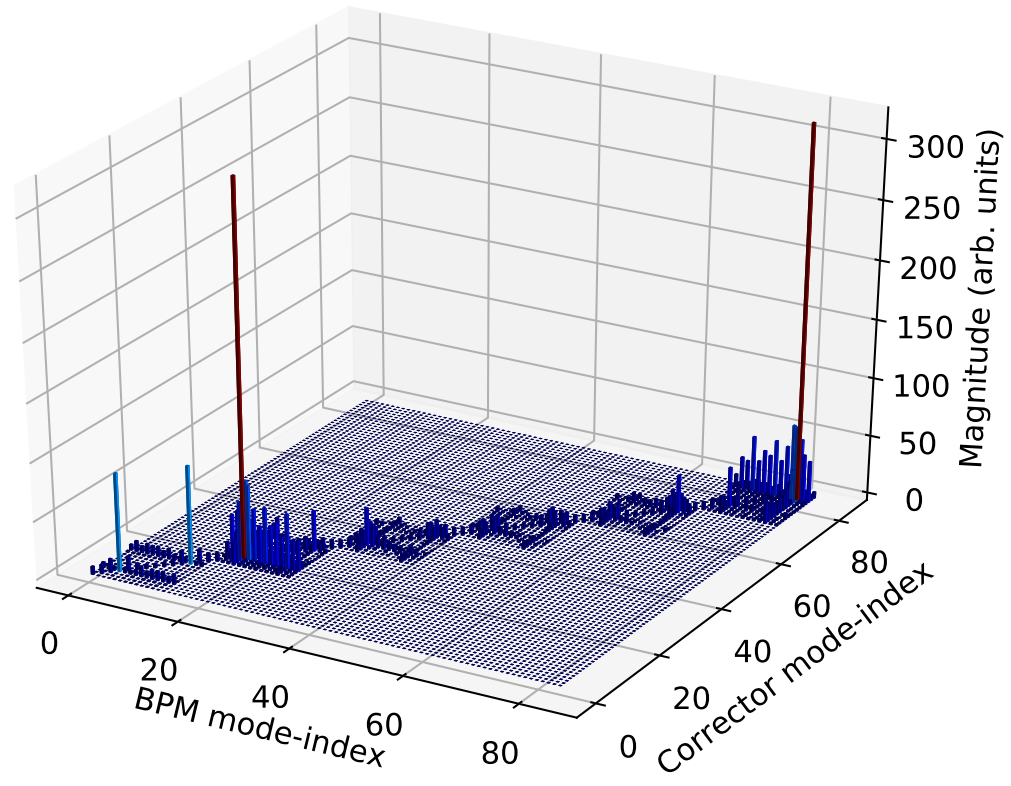

Figure 4.8 Magnitudes of the complex entries of the block diagonal matrix D defined in Eq. 4.38, calculated for the case of ignoring the beta beating in the $y$-plane of SIS100.

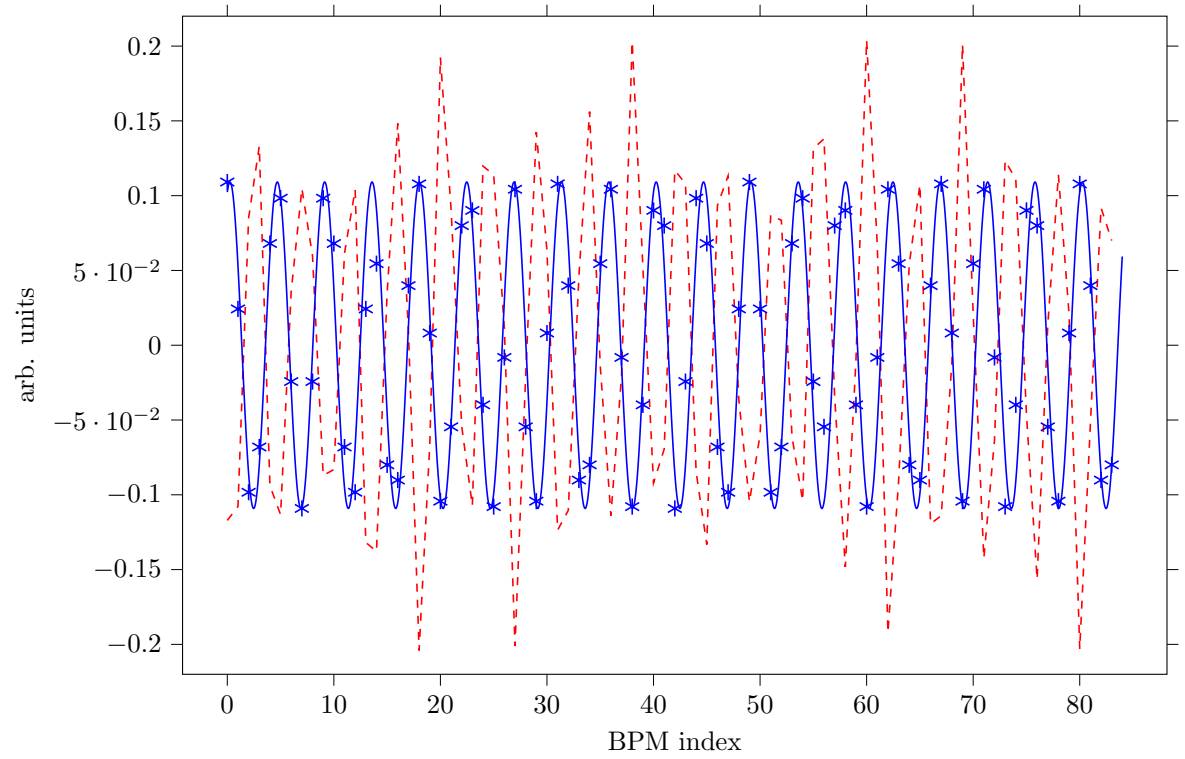

Figure 4.9 Tune modes of DFT matrix $\left(\mathbf{F}_{6}^{*} \otimes \mathbf{F}_{14}^{*}\right)$ (blue, solid) and SVD matrix $\mathbf{U}$ (red, dotted) for SIS100 ORM in the x-plane. The horizontal tune is 18.87. 



Figure 4.10 Comparison of the SVD singular values with the magnitudes of the rows of matrix D defined in Eq. 4.38 for the SIS100 ORM in x-plane. The abscissa is the index of singular values of the matrix $\mathbf{S}$ as well as the index of rows of matrix D (after sorting in the descending order of their magnitude).

\subsection{Advantages of symmetry exploitation}

Symmetry exploitation in the ORM for the DFT-based decomposition has some practical advantages over SVD-based decomposition. In this section, the benefits concerning robustness against "missing" BPMs (e.g. ignoring a possibly faulty position reading), computational complexity and removal of dispersion induced effect from the closed orbit are demonstrated with the help of simulations and experiments for the SIS18 synchrotron of GSI. The application of circulant symmetry in the uncertainty modeling for SIS18 COFB system is presented in next chapter, in detail.

\subsubsection{Orbit correction in the case of malfunctioning BPMs}

The physical interpretation of BPM and corrector mode space provided by DFT-based decomposition of the ORM can be used to interpolate the closed orbit at the location of some malfunctioning or "missing" BPM. This is demonstrated for the SIS18 in vertical plane using MAD-X for a scenario of two consecutive BPMs being excluded. The operational scenario of BPM electronics failure due to radiation shower can happen in hadron synchrotrons and leads to local bumps if there is not enough redundancy in the number of BPMs. Figure 4.11 (top) shows a simulated closed orbit (red curve) perturbed as a result of 

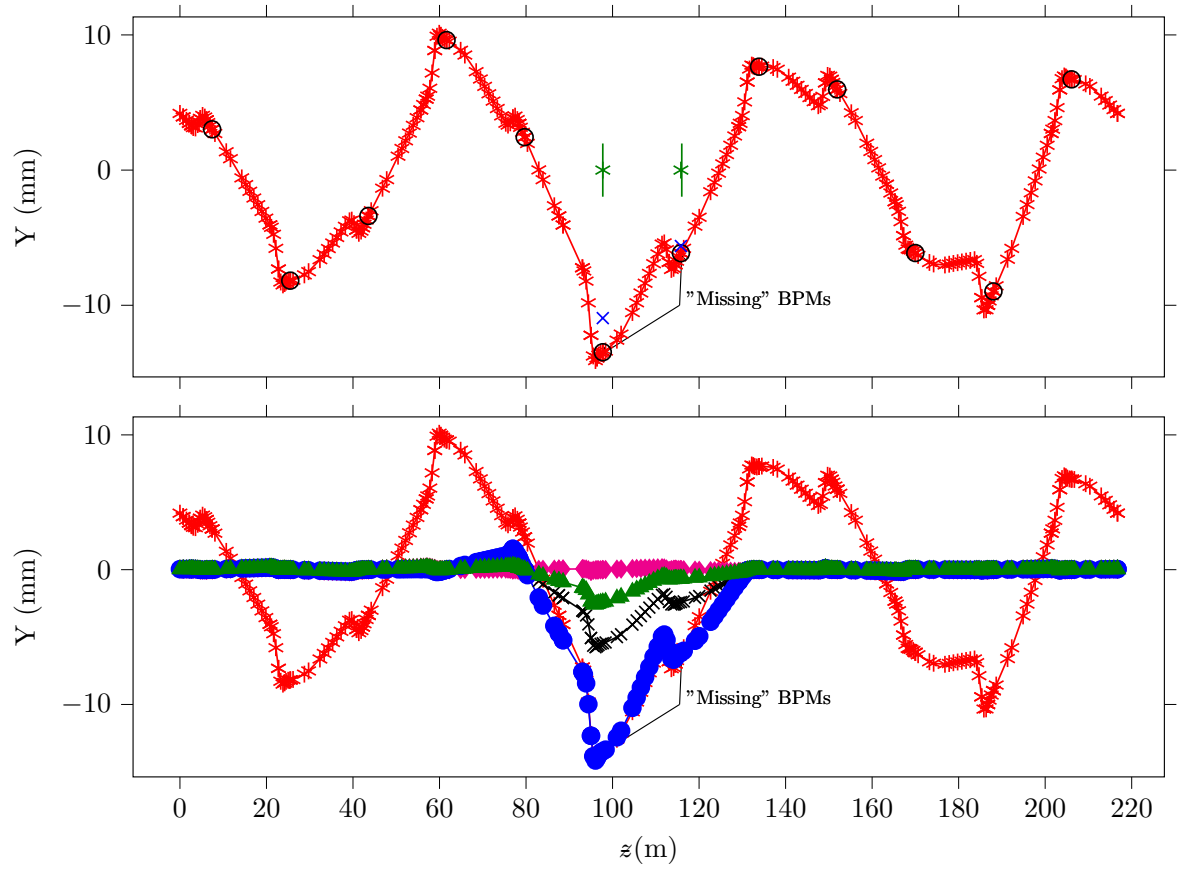

Figure 4.11 Top: Prediction of the closed orbit position at the "missing" BPM locations. $\operatorname{Red}\left({ }^{(*}\right)$ : Simulated perturbed orbit in MAD-X in the y-plane of SIS18. Black('o'): Sampling of perturbed orbit at BPM locations. Green('*'): Random initial guess for the orbit position at the two "missing" BPM locations. Blue(' $\mathrm{x}$ '): Estimated orbit position at "missing" BPM locations using DFT mode structure of the ORM. Bottom: Simulation of orbit correction for various cases of "missing" BPMs. $\operatorname{Red}\left({ }^{(*}\right)$ : Simulated perturbed orbit in MAD-X in y-plane of SIS18. Magenta(' $\left.{ }^{\prime}\right)$ : Corrected orbit using all BPMs. Green(' $\lambda$ '): Corrected orbit using the predicted orbit position for the "missing" BPMs. Black('x'): Corrected orbit excluding the rows corresponding to the "missing" BPMs from ORM. Blue('o'): Corrected orbit by taking zero orbit position at the "missing" BPM locations.

random vertical misalignment (in a range of -0.85 to $0.85 \mathrm{~mm}$ ) in all 24 quadrupoles of the SIS18. A misaligned quadrupole has the effect of a magnetic dipole on the beam and as a result the closed orbit is perturbed from its "ideal path". The black dots in 4.11 (top) represent the sampling of the perturbed orbit at the locations of BPMs while the green dots show the mean values of random orbit positions (in a range of -1 to $1 \mathrm{~mm}$ marked as error bars around the green dots) assumed at the location of "missing" or faulty BPMs. A combination of cosine functions with discrete frequencies $f=3,4,2,5$ (corresponding to dominant Fourier modes) was used to estimate the orbit position at the "missing" BPM locations keeping their relative amplitudes and phases as free parameters to be optimized. The fitting algorithm was constrained to keep the fitted curve closest to the orbit positions within an accuracy of 0.01 
$\mathrm{mm}$ at the working BPM locations while free to choose any value at the location of "missing" BPMs. As a result, the optimized orbit positions are found to be closer to the actual orbit positions within maximum errors of $3 \pm 0.048 \mathrm{~mm}$ and $0.5 \pm 0.048 \mathrm{~mm}$, respectively. Figure 4.11 (bottom) shows the simulated orbit correction using the estimated orbit positions (green curve). The red curve shows the perturbed orbit without corrections while the magenta curve shows the corrected orbit when all BPMs are working. The orbit correction using SVD of the non circulant matrix (excluding the rows in the ORM corresponding to the faulty BPMs) is plotted in black. The orbit correction taking the orbit position "zero" at the "missing" BPM locations and using a circulant matrix is also plotted as a blue curve for comparison. The robustness against "missing" BPMs is shown by the overall improved correction obtained using an estimated beam position instead of using the non circulant matrix. Besides the better global correction one can also get the benefits of circulant symmetry and DFT-based decomposition (e.g. online decomposition during ramp) even when the symmetry has been broken due to the "missing" BPMs.

\subsubsection{Computational complexity}

DFT-based diagonalization and inversion of the ORM has significant computational benefit over SVD. For a square matrix of dimension $n$, the numerical complexities for the SVD of the whole matrix and the DFT of its first row are $\mathcal{O}\left(4 n^{3}\right)[78]$ and $\mathcal{O}\left(n^{2}\right)$ [41], respectively. Such a reduction in numerical complexity becomes meaningful for larger ORMs and for synchrotrons having a continuously changing ORM within a cycle and also from one cycle to another. SIS18 is an example (sec. 3.2) of the latter, where lattice settings as well as ORM changes systematically during the ramp. In such cases, the possibility of online DFT-based ORM inversion becomes attractive from the perspective of computational speed as well as storage and transfer of the inverted ORMs.

\subsubsection{Momentum mismatch and orbit correction}

A mismatch between RF frequency and the dipole field results in a relative mismatch in the average momentum of the beam $\frac{\Delta p}{p}$ and a closed orbit deviation from the equilibrium position primarily in the $\mathrm{x}$-plane (Eq. 2.15), in the absence of coupling is given as,

$$
\Delta x_{D}(s)=D_{x}(s) \frac{\Delta p}{p}
$$

Here $D_{x}(s)$ is the dispersion function and $\Delta x_{D}(s)$ is the resultant shift in the closed orbit. Fig. 4.12 shows a set of measured horizontal dispersion orbits for a relative momentum mismatch in the range of $-2 \%$ to $2 \%$ with a step size of $0.5 \%$ in SIS18 at extraction energy. One can see a shift of the mean value of the closed orbit as a function of $\frac{\Delta p}{p}$. An attempt to correct such an orbit shift can saturate the corrector magnets. Therefore, the contribution from dispersion needs to be subtracted from the closed orbit before correction. Generally, the dispersion-induced orbit distortion will couple into many modes of BPM space, depending on the sampling of the dispersion function.

For the SIS18, the dispersion function has the same value $D(s)=D_{0}$ at all the BPM locations 


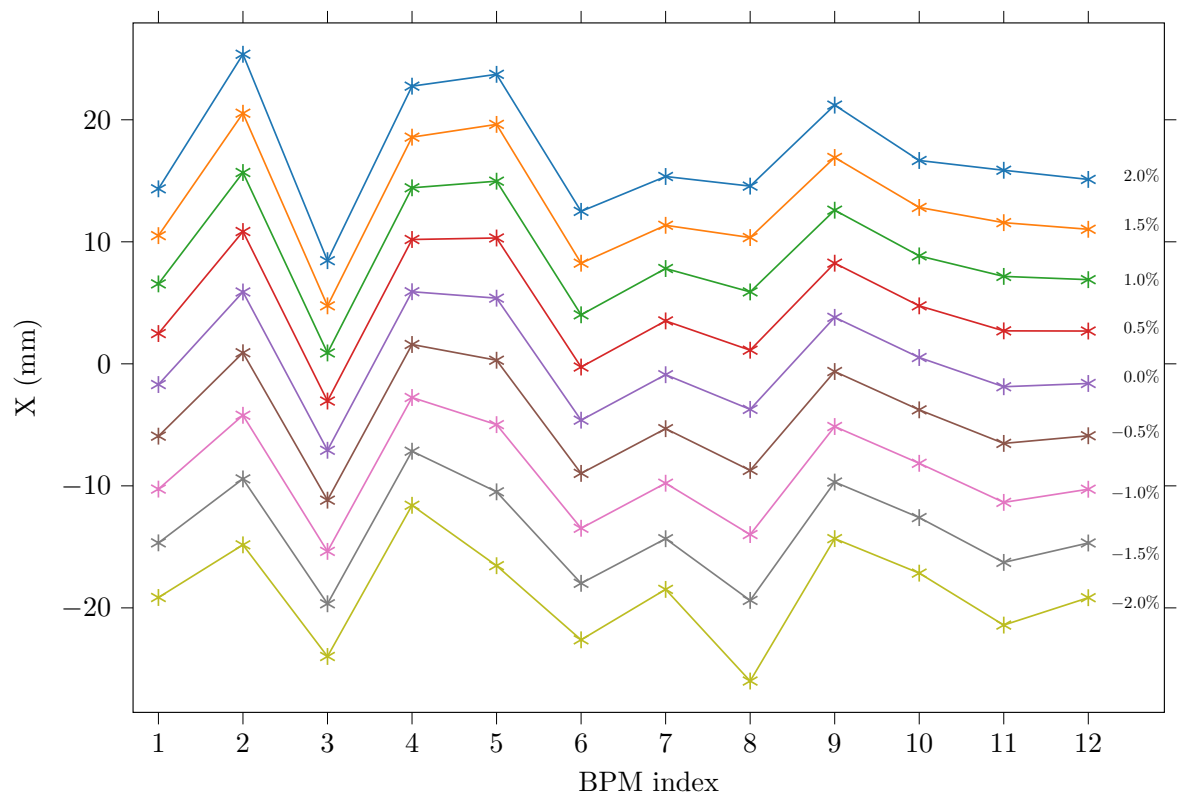

Figure 4.12 Measured horizontal closed orbits in SIS18 for the various average momentum offsets represented as \% with each orbit.

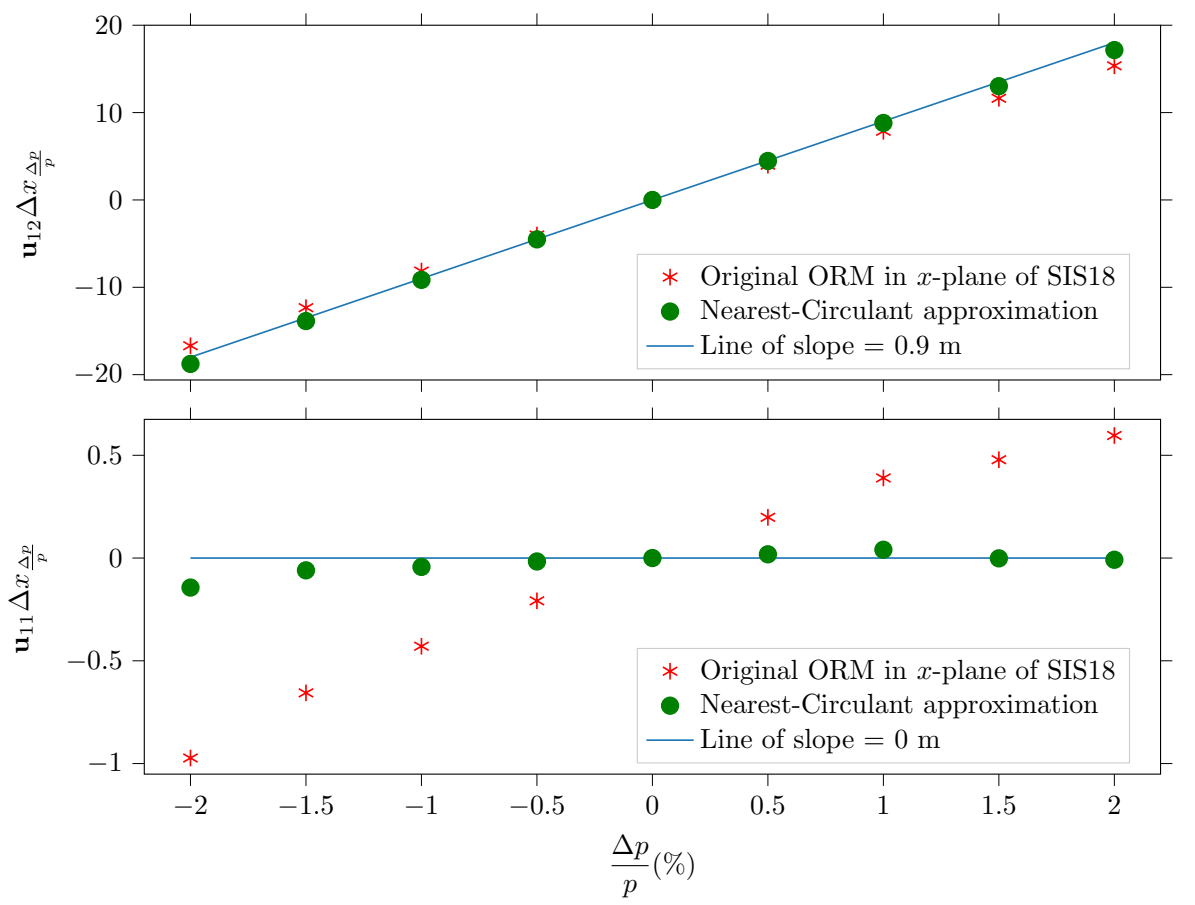

Figure 4.13 Coupling of experimentally measured DC shift $\Delta x(\mathrm{~mm})$ in the horizontal closed orbit to the last two columns of the $\mathbf{U}$ matrices $\left(\mathbf{u}_{12}\right.$ and $\left.\mathbf{u}_{11}\right)$ of the original ORM and its nearest-circulant approximation. 
and the resultant dispersion-induced DC part of the closed orbit will couple to the pure DC mode of the BPM space corresponding to $f=0$ in case of a circulant ORM and can be ignored by removing the singular value corresponding to that mode (the last column of $\mathbf{U}$ ). Fig. 4.13 shows the coupling of the DC part of the measured closed orbit to the last two modes of the original non circulant ORM and its nearest-circulant approximation for SIS18, in $x$-plane. It is evident that the DC shift of the closed orbit couples to the last mode of the matrix $\mathbf{U}$ as $\mathbf{u}_{12} \Delta x_{\frac{\Delta p}{p}}$ increases linearly with $\frac{\Delta p}{p}$ with a slope of $0.9 \mathrm{~m}$ (equal to the value of dispersion function $D_{0}$ at the BPM locations). The slight deviation from this behavior in case of the original ORM is due to a slight distortion of last mode from pure DC structure due to the broken symmetry (Fig. 4.2). This results in the coupling of the DC part of the closed orbit to higher modes as well, as evident in Fig. 4.13. Here in case of nearest-circulant approximation there is almost zero coupling to the $2^{\text {nd }}$ last mode. This shows the benefit of circulant symmetry in the ORMs in order to reject energy dispersion in the closed orbit by simply discarding the last DC mode of the matrix $\mathbf{U}$, instead of measuring and subtracting it from the closed orbit before correction. The projection of dispersion into the last mode of $\mathbf{U}$ can also be utilized to synchronize the rf frequency and the dipole field.

\subsection{Summary and contributions}

The contributions of the work presented in this chapter can be summarized below, while some of them are also published in [79].

- Singular value decomposition has some limitations in terms of the physical interpretation of mode space of the orbit response matrix, besides being an efficient technique for the matrix diagonalization and inversion.

- For synchrotrons where the placement of BPMs and correctors is periodic in terms of the lattice parameters, an efficient method relying on exploitation of the circulant symmetry for the diagonalization and inversion of the orbit response matrix (ORM) is introduced.

- The block-circulant symmetry arising from the block-wise periodicity of a lattice is shown to have SVD-like robustness benefits in line with the pure circulant symmetry case under DFT decomposition.

- A circulant ORM can be decomposed into BPM and corrector vector spaces with the help of a one dimensional DFT; this method is significantly faster than the singular value decomposition (SVD) technique and provides physical interpretation of the mode space. A relation between DFT coefficients and SVD singular values as well as modes is discussed which shows that DFT-based decomposition contains the benefits of both harmonic analysis and SVD.

- DFT-based decomposition provides information compression into the diagonal matrix only, which can significantly simplify uncertainty modeling in the ORM, overcoming a deficiency of SVD decomposition where the information is distributed among all three matrices. 
- For synchrotrons where the orbit response matrix drifts away from the pure symmetric structure, a nearest-circulant or nearest-block-circulant approximation has been introduced hence making the method applicable to a wider range of synchrotrons.

- The usefulness of the transparent interpretation of BPM and corrector spaces as Fourier modes is demonstrated for the SIS18 synchrotron at FAIR by interpolating the closed orbit to the location of "missing" BPMs to achieve a better global orbit correction.

- The reduced computational complexity of DFT can have benefits concerning the overall performance of closed orbit feedback systems in case of large ORMs and during the energy-ramp orbit correction.

- The rejection of the dispersion-induced orbit shift during orbit correction is demonstrated with measurements at SIS18, as a benefit of circulant symmetry exploitation.

- The DFT-based method of closed orbit correction is presented in the context of the FAIR synchrotrons, but would apply to any synchrotron or storage rings with the aforementioned symmetry or a slightly broken symmetry. Thus the importance of symmetry exploitation in the ORM is emphasized for the consideration at the design stage of the closed orbit feedback system. 


\section{Chapter Five}

\section{Characterization of spatial model mismatach for a COFB system}

This chapter is dedicated to the characterization of closed orbit feedback (COFB) systems in the light of control theory, for the spatial model mismatch between the system and the controller. COFB systems are classified into slow and fast regimes based upon the update rate of correction-strengths. A stability criterion is worked out for both regimes which gives a practical margin of spatial model mismatch consistent with measurements, in comparison to already used small gain theorem. Simulations are performed for various sources of model mismatch in SIS18 for the stability and performance of COFB system and the theoretical results are verified experimentally at COFB system of COSY Jülich.

\subsection{Closed orbit feedback system in generic form}

In chapter 2, the subsystems of the closed orbit feedback system along with their transfer functions are described in detail. Here, we extend the discussion to the closed-loop representation of the closed orbit feedback (COFB) system which in general is more regulator problem than the servo problem (sec. 2.8) as the set value is mostly fixed in the form of golden orbit and COFB system has to reject external disturbances acting on the closed orbit. The important terms and symbols from chapter 2 which are later used in this chapter are revised here as,

- $g(s) e^{-\tau_{d} s}$ is the temporal part of system model in Laplace domain where $\tau_{d}$ is the pure time delay. $\mathbf{R}$ represents the ORM of the synchrotron and the full system model $\mathbf{G}(s)$ is defined as $g(s) e^{-\tau_{d} s} \mathbf{R}$. (sec. 2.8.3)

- $\mathbf{R}_{\Theta}^{+}$is the pseudo-inverse of the ORM used for the calculation of corrector-strength vector $\boldsymbol{\Theta}$ for a perturbed orbit vector $\mathbf{w}$. The full controller transfer function $\mathbf{K}(s)$ is defined as $k(s) \mathbf{R}_{\boldsymbol{\Theta}}^{+}$. (sec. 2.7.4 and 2.8.4.

- SISO (single-input-single-output) system has only one sensor and one actuator while COFB system is an example of MIMO (multiple-input-multiple-output) systems where $M$ number of BPMs are used for the closed orbit measurement and $N$ number of correctors are used for the orbit correction. (sec. 2.8.2 


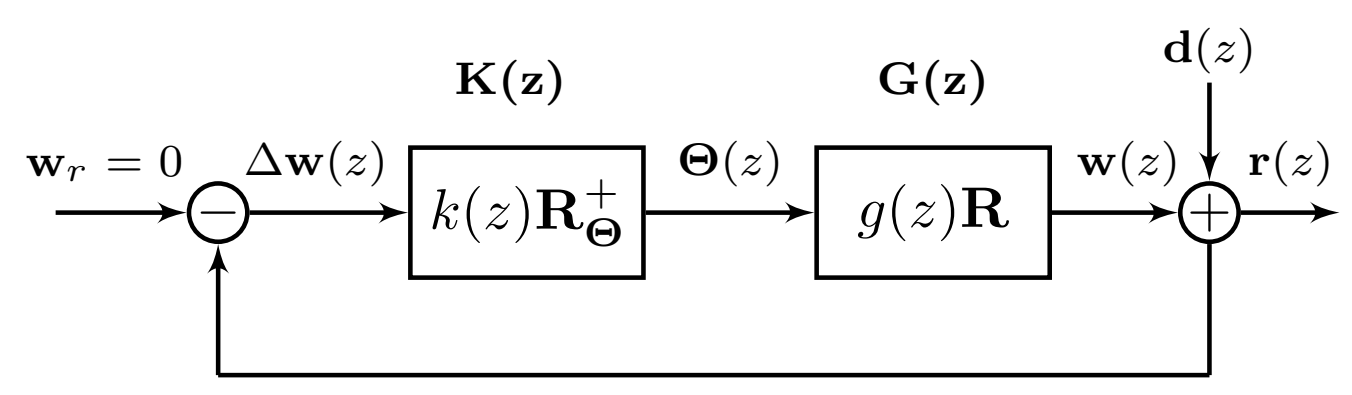

Figure 5.1 Schematic of closed orbit feedback loop in z-domain.

- Sensitivity function characterizes the effect of closed-loop on the disturbance rejection and is defined in Laplace domain $s$ for a MIMO system as $\mathbf{S}(s) \triangleq[\mathbf{I}+\mathbf{G}(s) \mathbf{K}(s)]^{-1}$. (sec. 2.8.2). The lowest frequency where $|\mathbf{S}(s)|$ has magnitude 1 is called sensitivity crossover frequency $f_{s c}$

- The digital feedback controllers are implemented in discrete time $z$-domain whose transformation with Laplace domain is given as $z=e^{s T_{s}}$ where $T_{s}$ is the sampling period. (sec. 2.8.5).

The schematic description of an arbitrary feedback system shown in Fig. 2.8 can be individualized for COFB system as shown in Fig. 5.1 where the control system $k(z)$ (where $z$ represents the $z$-domain variable) is implemented in "sample and hold" mode such that the beam position is measured at discrete time steps $\left\{t=k T_{s}, k \in \mathbb{Z}^{+}\right\}$with $T_{s}$ being the sampling time interval and the actuator (corrector magnets) inputs are held constant over the time interval $t \in\left\{k T_{s},(k+1) T_{s}\right\}$. The dynamic part of the system model $g(s) e^{-\tau_{d} s}$ can also be represented in the zero-order hold and sampled discrete-time form with the help of modified $z$-transform $\left(\mathcal{Z}_{m}\right)$ (defined in sec. 2.8.5) as,

$$
g(z)=z^{-l}(z-1) \mathcal{Z}_{m}\left[\frac{g(s)}{s}\right]
$$

where $l$ is the smallest positive integer satisfying $l T_{s} \geq \tau_{d}$. Consequently, the required corrector settings $\Theta(z)$ for a given perturbed orbit vector $\Delta \mathbf{w}(z)$ are calculated as,

$$
\boldsymbol{\Theta}(z)=k(z) \mathbf{R}_{\Theta}^{+} \Delta \mathbf{w}(z)
$$

For the servo problem, the sensitivity function from disturbance $\mathbf{d}(z)$ to the output $\mathbf{r}(z)$ is a good parameter of characterization for the performance of the closed-loop and can be written for the Fig. 5.1 as,

$$
\mathbf{S}(z) \triangleq\left[\mathbf{I}+g(z) \mathbf{R} k(z) \mathbf{R}_{\Theta}^{+}\right]^{-1}
$$

The order of the total system model $g(s)$ for the COFB system depends upon the dynamics of the individual subsystems and the system identification (measurement of the transfer function of the full system) is a necessary step before designing the controller. But in general, 
a first order approximation for $g(s)$ of bandwidth $a(\mathrm{rad} / \mathrm{s})$ is a workable choice for most of the synchrotrons (e.g. [3]) such that

$$
g(s)=\frac{a}{s+a} e^{-\tau_{d} s}
$$

where the corrector frequency response is usually the most dominant contributor in the system model. The corresponding discrete domain transfer function for the system model of Eq. 5.4 (with the help of Eq. 5.1) is given as,

$$
g(z)=z^{-l} \frac{b_{0} z+b_{1}}{z-a_{1}}
$$

where $l$ is the delay in the units of sampling time $T_{s}, a_{1}=e^{-a T_{s}}, b_{0}=1-e^{a\left(\tau_{d}-l T_{s}\right)}$ and $b_{1}=e^{a\left(\tau_{d}-l T_{s}\right)}-e^{-a T_{s}}$.

Similarly, the type and order of the controller $k(z)$ depends upon the identified system model and two kinds of controllers have been reported in the literature of COFB systems so far: Proportional-Integral (PI) controller [36] and the internal model controller (IMC) [3], as introduced already in chapter 2 and the system identification for SIS18 will come later in subsequent chapters.

\subsection{Spatial model mismatch}

A mismatch between the actual ORM $\mathbf{R}$ of the synchrotron and its model $\mathbf{R}_{\Theta}$ used in the controller is always probable and can have a detrimental effect on the performance and the stability of the COFB system. The possible reasons for such a model mismatch fall into three broad categories:

- Uncertainty in the measurement of the orbit response matrix due to BPM and corrector calibration errors 65,80$]$.

- Change in the machine ORM relative to the controller ORM due to systematic drift of the machine settings during operation. SIS18 is an examples in which the triplet to doublet transition of the quadrupole configuration over the ramp results in the ORM variation. This is already shown in Fig. 3.3 (bottom) where the highest singular values of the calculated ORMs using MAD-X in both planes for the quadrupole settings of a typical ramp of $10 \mathrm{~T} / \mathrm{s}$, are plotted.

- Changes of beam parameters where coherent tune shift being the most popular one and relevant for both hadron machines [53] as well as for synchrotron light sources [3, 65]. Typical sources of tune shift include magnet current drifts during long storage of the beams, magnet gradient errors during the fast ramps [81, 82 and intensity-dependent tune shifts at higher beam intensities [55] (Fig. 3.5).

In order to implement the fast COFB system for SIS18 in the presence of various kinds of systematic as well as beam parameter assisted changes in the ORM, following questions needed to be answered: 
1. In order to achieve a persistent orbit correction during the ramp, when the machine ORM changes over time, how frequently the ORM in the controller needs to be updated?

2. If the COFB system has to run in the fixed-ORM mode during the ramp, is it possible to use the fixed controller parameters? If not, how should they scale with the machine ORM variation over the ramp in order to avoid controller instability?

3. Variation of which lattice parameters is crucial e.g. tune (global lattice parameter) or beta function at the location of BPMs and correctors (local lattice parameter) (see Eq. 2.14) and how can their influence be characterized?

4. How much intensity dependent tune shifts can be tolerated by COFB system?

Surprisingly, these questions could not be answered based upon previous work related to the uncertainty modeling in the ORM, found in literature. The primary focus has been defining the stability margins from the small gain theorem [80, 83] which yields a conservative stability criterion while the ORM variation in SIS18 is found much larger that the limits defined by small gain theorem. Another recent work is based on enhancement of the stability margins (at the cost of bandwidth) for higher-order modes of the ORM using the Tikhonov regularization [3] (Eq.4.25). Therefore, a thorough study of the COFB robustness against the large spatial model mismatch is required before the realization of SIS18 COFB system. A clear motivation is to establish the theoretical stability margins consistent with the simulation and experimental results against the various sources of model mismatch. It is however important to note that, if the time scale of the ORM variation due to aforementioned effects is longer (an order of magnitude) than the delays and time constants of the temporal response of BPMs and corrector magnets, the ORM can still be considered as a separate part $\mathbf{R}$ in the total system model $\mathbf{G}(s)$. Therefore the ORM variations are treated exclusively as the spatial model mismatch in this thesis.

The orbit correction systems are divided into two temporal regimes, based upon the relative time scale of the correction rate (reciprocal of time step for update of controller output) and the dynamics of the system.

- Slow regime: For the slow feedback systems, the time step of orbit correction is at least an order of magnitude longer than than system delays and open-loop time constant (the time required to achieve $63.2 \%$ of the steady-state). The performance of a COFB system against the spatial model mismatch is primarily evaluated by the correction ratio in the slow regime defined as the ratio of residual orbit to original orbit (RMS) after one correction.

- Fast regime: Systems, where the correction rate is comparable to temporal dynamics are referred to here as the fast feedback systems. In this regime, the performance of the COFB system is evaluated in terms of sensitivity crossover frequency defined in sec. 5.1 .

The motivation for this classification is to first ignore the temporal parts of the system and the controller in the slow regime in order to define stability criteria only in terms of the ORMs and then modify the results for the scenario of including temporal parts, in the fast regime. 


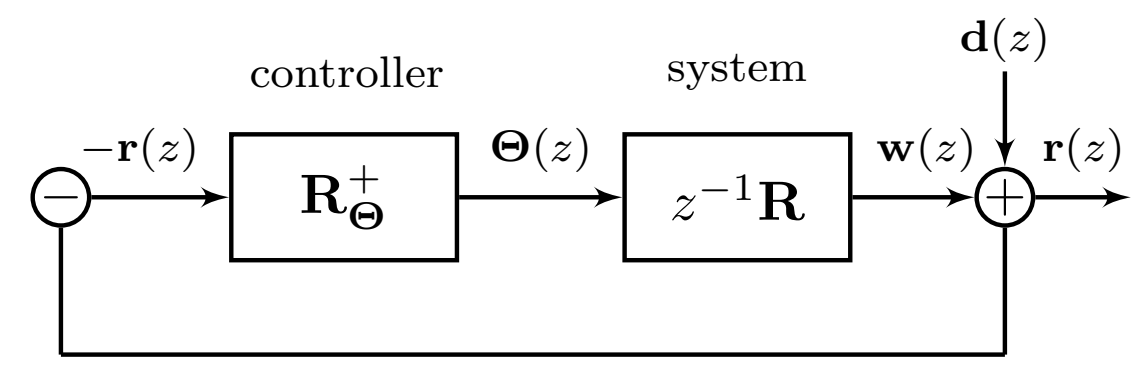

Figure 5.2 Schematic of the digital closed orbit feedback system in the slow regime. $-\mathbf{r}(z)$ is the error signal, $\boldsymbol{\Theta}(z)$ is the corrector settings, $\mathbf{r}(z)$ is the residual orbit and $\mathbf{d}(z)$ is the disturbance defined in Eqs. 2.3 to 2.5 .

\subsection{Spatial model mismatch in slow temporal regime}

The slow temporal regime of orbit correction is a scenario where the temporal dynamics of the system (corrector magnets and their power supplies etc.) is fast enough to be ignored as compared to the rate of update of the controller output. An example is the correction of a static orbit distortion caused by a fixed non-oscillating magnet field error in a synchrotron. In this case, the sampling time $T_{s}$ can be selected in such a way that the effect of each controller output is fully settled and the system is in the steady-state before the next corrector input. The orbit correction for low frequency perturbations with fast corrector magnets (having bandwidth an order of magnitude higher than frequency of interest) can also be categorized in slow regime of orbit correction. The closed orbit feedback system in the slow regime is implemented in a way that the effect of corrector settings $\boldsymbol{\Theta}_{k}$ calculated at a given time step $\left\{t=k T_{s}, k \in \mathbb{Z}^{+}\right\}$corresponding to the perturbed orbit of previous time step $\mathbf{r}_{k-1}$ is added to $\mathbf{r}_{k-1}$ i.e.,

$$
\mathbf{r}_{k}=\mathbf{r}_{k-1}+\mathbf{R} \Theta_{k}
$$

where $\boldsymbol{\Theta}_{k}=-\mathbf{R}_{\Theta}^{+} \mathbf{r}_{k-1}$. Consequently $\mathbf{r}_{k}$ can be written as

$$
\mathbf{r}_{k}=\mathbf{r}_{k-1}-\mathbf{R} \mathbf{R}_{\Theta}^{+} \mathbf{r}_{k-1}=\left(\mathbf{I}-\mathbf{R} \mathbf{R}_{\Theta}^{+}\right) \mathbf{r}_{k-1}
$$

Here, $\mathbf{I}$ is the identity matrix of the size of $\mathbf{R} \mathbf{R}_{\boldsymbol{\Theta}}^{+}$which is a square matrix even if $\mathbf{R}$ and $\mathbf{R}_{\Theta}^{+}$are rectangular matrices, since $\mathbf{r}_{k}$ and $\mathbf{r}_{k-1}$ are vectors of same dimension equal to the number of BPMs. Converting the difference Eq. 5.7 into z-domain, one obtains

$$
\mathbf{r}(z)=z^{-1} \mathbf{r}(z)-z^{-1} \mathbf{R} \mathbf{R}_{\Theta}^{+} \mathbf{r}(z)
$$

Since $g(s)=1$ and $\tau_{d} \ll T_{s}$ (for slow regime), following Eq. 5.1 $g(z)=z^{-1}$ and $k(z)=1$ and for a constant initial perturbation, the residual from previous time step acts as the disturbance to be removed at the current time step such that $\mathbf{d}(z)=z^{-1} \mathbf{r}(z)$. The corresponding block- 
diagram is shown in Fig. 5.2 for which Eq. 5.8 can be re-written as

$$
\begin{aligned}
\mathbf{r}(z) & =\mathbf{d}(z)-z^{-1} \mathbf{R} \mathbf{R}_{\Theta}^{+} \mathbf{r}(z) \\
& =\left[\mathbf{I}+z^{-1} \mathbf{R} \mathbf{R}_{\Theta}^{+}\right]^{-1} \mathbf{d}(z) \\
& =[\mathbf{I}+\mathbf{L}]^{-1} \mathbf{d}(z) \\
& =\mathbf{S}(z) \mathbf{d}(z)
\end{aligned}
$$

The term $\mathbf{S}(z)$ is by definition the sensitivity function matrix from disturbance to the output for the closed-loop of a MIMO system where $\mathbf{L}$ is the loop transfer function equal to the product of system and controller models (for definition see Eq. 2.70).

From Eq. 5.7, the residual orbit after $k$ time steps with respect to initial perturbed orbit $\mathbf{r}_{-1}=\mathbf{w}_{0}$ can be written as,

$$
\mathbf{r}_{\mathrm{k}}=\left(\mathbf{I}-\mathbf{R} \mathbf{R}_{\Theta}^{+}\right)^{k} \mathbf{w}_{0}
$$

It is evident from Eq. 5.10 that for the slow regime, the spatial model mismatch can be fully described by the following combined matrix structure referred to as "correction matrix",

$$
\mathbf{M}=\mathbf{I}-\mathbf{R R}_{\Theta}^{+}
$$

which will be a null matrix for a perfect model match i.e. for $\mathbf{R}_{\Theta}=\mathbf{R}$.

\subsubsection{Stability criteria for the slow regime of COFB system}

Equations 5.10 and 5.11 show that the matrix $\mathbf{M}$ has to be a convergent matrix for the residual orbit to approach zero after subsequent time steps. The condition of convergence is thus given as,

$$
\rho(\mathbf{M})<1
$$

where $\rho(\mathbf{M})$ is known as the spectral radius of matrix $\mathbf{M}$ and is defined as the largest absolute eigenvalue of $\mathbf{M}(\mathrm{A} .5 .3$ of 47 ) as,

$$
\rho(\mathbf{M})=\max _{i}\left\{\left|\lambda_{i}\right|\right\}
$$

Inequality 5.12 can be regarded as the general stability criteria for a COFB system in the slow regime against any source of spatial model mismatch as it depends only on the relative discord between $\mathbf{R}$ and $\mathbf{R}_{\Theta}$ and can be helpful to find the limits of optical parameter (e.g. tune) variations beyond which the correction will lead to the orbit divergence and the beam loss.

It is important to mention that pseudo-inverse $\mathbf{R}_{\Theta}^{+}$in Eq. 5.11 is often truncated for numerical stability and for robust closed orbit correction, which leads to a reduction in the rank of $\mathbf{R}_{\Theta}^{+}$. In this case, the matrix $\mathbf{R}$ also needs to be equally truncated before the calculation of $\rho(\mathbf{M})$ which otherwise will be misleadingly equal to 1 because of the truncated modes of $\mathbf{R}_{\Theta}^{+}$. 


\subsubsection{Small gain theorem}

Typically, the use of small gain theorem is found in literature for the stability margins of the COFB system [65, 80] which is a sufficient condition for the closed-loop stability but yields very stringent margins. According to the theorem, consider a system with a stable loop transfer function matrix $\mathbf{L}(\mathbf{s})$, then the closed-loop system is stable if

$$
\|\mathbf{L}(j \omega)\|<1 \quad \forall \omega
$$

where $\omega$ is the imaginary part of Laplace frequency $s$ and the symbol $\|\mathbf{L}(j \omega)\|$ denotes any matrix norm satisfying $\|\mathbf{A B}\| \leq\|\mathbf{A}\| \cdot\|\mathbf{B}\|$. The $\mathbf{H}_{\infty}$ norm $\|\cdot\|_{\infty}$ defined as the highest singular value of the matrix (Sec. 4.10 .2 of 47$]$ ) can be used and has a good relevance for the ORM. An implication of the above statement is that if two subsystems $\mathbf{A}$ and $\mathbf{B}$ are connected in a feedback loop, then the closed-loop system is input-output stable if $\|\mathbf{A}\| \cdot\|\mathbf{B}\|<1$.

Application of small gain theorem for the COFB system relies on modeling the uncertainty of the system model as a separate matrix $\Delta$ and modeling rest of MIMO system as e.g. $\mathbf{T}$ for which the condition of stability given by small gain theorem for the interconnection of Fig. 5.3 is given as

$$
\|\Delta\|_{\infty} \cdot\|\mathbf{T}\|_{\infty}<1
$$

\subsubsection{Comparison between spectral radius condition and small gain theorem}

Here we make a theoretical comparison between small gain theorem and the spectral radius condition discussed in inequality 5.12 based upon following assumptions: 1) we consider circulant or block-circulant ORMs (a scenario which exists for FAIR synchrotrons and also some synchrotron light sources as discussed in previous chapter) for which the spectral radius is equal to the $\mathbf{H}_{\infty}$ norm i.e. the highest singular value $\bar{\sigma}$. 2) we consider only the well conditioned ORMs for which the general inverse exists such that $\mathbf{R}_{\Theta} \mathbf{R}_{\Theta}^{+}=\mathbf{I}$. 3) we assume only the additive uncertainty in the ORM in order to deal with ORM errors arising from optical parameter variations e.g. tune and beta function. It is important to mention that these assumptions are required only for a clear comparison between two methods. The spectral radius condition itself holds also for non-quadratic and non-circulant ORMs as the matrix $\mathbf{M}$ is always a square matrix. For errors of additive nature in ORM, the small gain theorem-based stability criterion has been worked out in 80 and ignoring temporal part (i.e. taking $\mathbf{T}=\mathbf{R}_{\Theta}^{+}$), it can be written as

$$
\begin{aligned}
& \bar{\sigma}\left(\Delta_{R}\right)<\frac{1}{\bar{\sigma}\left(\mathbf{R}_{\Theta}^{+}\right)} \\
& \bar{\sigma}\left(\Delta_{R}\right)<\underline{\sigma}\left(\mathbf{R}_{\Theta}\right)
\end{aligned}
$$

where $\Delta_{R}=\mathbf{R}-\mathbf{R}_{\Theta}$ and $\underline{\sigma}$ is the smallest singular value. The spectral radius stability condition shown in inequality 5.12 can also be solved in a similar manner for circulant symmetric ORMs as,

$$
\rho\left(\mathbf{I}-\mathbf{R R}_{\Theta}^{+}\right)=\rho\left(-\Delta_{R} \mathbf{R}_{\Theta}^{+}\right)=\rho\left(\Delta_{R} \mathbf{R}_{\Theta}^{+}\right)=\bar{\sigma}\left(\Delta_{R} \mathbf{R}_{\Theta}^{+}\right)<1
$$




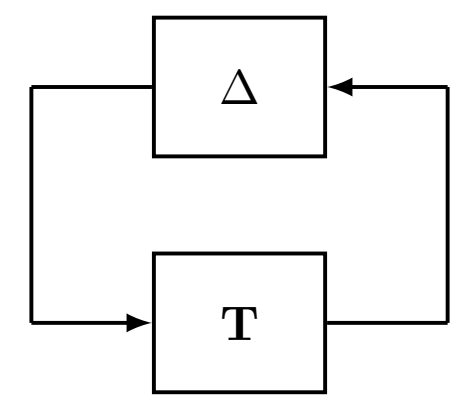

Figure 5.3 System interconnection for the application of small gain theorem.

Using the singular value inequality $\bar{\sigma}(A) \underline{\sigma}(B) \leq \bar{\sigma}(A B)($ A.3.4 of 47$])$,

$$
\begin{aligned}
\bar{\sigma}\left(\Delta_{R}\right) \underline{\sigma}\left(\mathbf{R}_{\Theta}^{+}\right)<\bar{\sigma}\left(\Delta_{R} \mathbf{R}_{\Theta}^{+}\right) & <1 \\
\bar{\sigma}\left(\Delta_{R}\right) & <\frac{1}{\bar{\sigma}\left(\mathbf{R}_{\Theta}^{+}\right)} \\
\bar{\sigma}\left(\Delta_{R}\right) & <\bar{\sigma}\left(\mathbf{R}_{\Theta}\right)
\end{aligned}
$$

According to Eq. 5.15, the highest allowed uncertainty is limited by the smallest singular value of the nominal ORM in case of small gain theorem while the spectral radius condition of Eq. 5.16 yields a much higher margin i.e. of the highest singular value. The ratio between the two margins is exactly the condition number of the nominal ORM for the circulant symmetry case.

\subsubsection{Measurement of the spectral radius}

Let us define the correction ratio $\delta_{i}$ as the ratio between the RMS of the residual orbit vector after $i^{\text {th }}$ iteration and the orbit vector after $(i-1)^{t h}$ iteration such that $\delta_{1}$ (after first iteration) is given as,

$$
\delta_{1}=\frac{\mathbf{r}_{1, \mathrm{RMS}}}{\mathbf{w}_{0, \mathrm{RMS}}}
$$

The $\mathbf{H}_{\infty}$ norm (highest singular value) of matrix $\mathbf{M}$ of Eq. 5.11 can be defined and related to the correction ratio as (Sec. 3.3.2 of [47])

$$
\|\mathbf{M}\|_{\infty}=\max \frac{\left\|r_{1}\right\|_{2}}{\left\|w_{0}\right\|_{2}} \geq \frac{\mathbf{r}_{1, \mathrm{RMS}}}{\mathbf{w}_{0, \mathrm{RMS}}}
$$

where $\|\cdot\|_{2}$ is the Euclidean norm ${ }^{1}$ and the symbol max means that the ratio is between those modes of $r_{1}$ and $w_{0}$ which have maximum coupling, i.e. which couple through the highest singular values. For the circulant symmetry, $\rho(\mathbf{M})=\|\mathbf{M}\|_{\infty}$ and the measured correction ratio can be related with $\rho(\mathbf{M})$ as

$$
\rho(\mathbf{M}) \geq \delta_{1}
$$

\footnotetext{
${ }^{1}$ defined as $\|x\|_{2}=\sqrt{x_{1}^{2}+x_{2}^{2}+\ldots . x_{n}^{2}}$
} 
For non-circulant ORMs, $\rho(\mathbf{M}) \leq\|\mathbf{M}\|_{\infty}$ (A.5.3 of $\left.[47]\right)$ and the correction ratio is asymptotically related to $\rho(\mathbf{M})$ by Gelfand's formula 84

$$
\rho(\mathbf{M})=\lim _{k \rightarrow \infty}\left\|\mathbf{M}^{k}\right\|_{\infty}^{\frac{1}{k}}
$$

as

$$
\rho(\mathbf{M}) \geq \lim _{k \rightarrow \infty} \frac{\mathbf{r}_{\mathrm{k}, \mathrm{RMS}}}{\mathbf{r}_{\mathrm{k}-1, \mathrm{RMS}}}=\lim _{k \rightarrow \infty} \delta_{k}
$$

In general, an approximation $\rho(\mathbf{M})=\frac{\mathbf{r}_{1, \mathrm{RMS}}}{\mathbf{w}_{0, \mathrm{RMS}}}$ can be made if the largest effect of model mismatch is on the dominant mode in correction space. As discussed in the next sections, this is a reasonable approximation in practical situations.

\subsection{Spectral radius for circulant approximations}

In chapter 4 a method for approximating an ORM with broken symmetry to its nearestcirculant or nearest block-circulant matrix was introduced. It is important to mention that even if the method provides the "most" nearest symmetric approximation, the resultant matrix $\mathbf{R}_{N C}$ to be used in the controller has a certain magnitude of mismatch with respect to the the original ORM $\mathbf{R}$ of the synchrotron. The spectral radius condition given in inequality. 5.12 can be applied in order to quantify the mismatch caused by nearest (block-) circulant approximation which can be calculated for the correction matrix $\mathbf{M}$ in this case as,

$$
\rho\left(\mathbf{M}_{N C}\right)=\rho\left(\mathbf{I}-\mathbf{R} \mathbf{R}_{N C}^{+}\right)
$$

For example, for the horizontal plane of SIS18 the spectral radius for the nearest-circulant approximation is 0.13 for which the experimental orbit correction in Fig. 4.5 shows a difference of $9 \%$ as compared to the original ORM.

Similarly, the spectral radius of various kinds of symmetric approximations for SIS100 ORMs calculated using Eq. 5.21 in both planes are given in table 5.1. Relatively smaller value of $\rho(\mathbf{M})$ in case of nearest-block circulant approximation as compared to the nearest-circulant approximation is because of the fact that SIS100 ORMs are more closer to block circulant symmetry due to the existence of super periods in the lattice. On the other hand, one can conclude that any symmetric approximation for FAIR synchrotrons can be used in the slow regime, as the $\rho(\mathbf{M})$ satisfies inequality 5.12 for all cases.

Table 5.1 Calculated spectral radius in case of symmetric approximations for SIS100 ORMs.

\begin{tabular}{lll}
\hline \hline SIS100 ORM & $x$-plane & $y$-plane \\
\hline Original ORM & 0 & 0 \\
\hline Nearest-circulant approximation & 0.34 & 0.31 \\
\hline Nearest-block circulant approximation & 0.13 & 0.17 \\
\hline Ignoring beta-beating & 0.16 & 0.20 \\
\hline
\end{tabular}




\subsection{Spectral radius calculation for spatial model mismatch in SIS18: simulations}

As discussed in previous chapters, the SIS18 COFB system has to operate from injection till extraction including the acceleration ramp for the beam quality preservation before injection into SIS100. The triplet to double transition for quadrupole configuration and tune shifts due to quadrupole field errors as well as due to higher beam currents are the major sources of spatial model mismatch for SIS18 COFB system and need to be investigated for the stability margins.

In this section, we calculate the spectral radius and simulate the correction ratio in MAD-X as a function of model mismatch of various sources to estimate instability thresholds of the SIS18 COFB system, in the slow feedback regime. The ideas are later extended to the fast feedback regime in section 5.7. Following types of model mismatch are investigate:

- On-ramp ORM variation due to triplet to double transition.

- Tune variation over the ramp due to quadrupole field gradient error.

- Intensity dependent tune shift.

- Beta beating due to quadrupole field errors.

\subsubsection{On-ramp ORM variation for SIS18}

The calculated on-ramp ORM variation characterized by its largest singular value $(\bar{\sigma})$ is already shown for a typical dipole ramp of $10 \mathrm{~T} / \mathrm{s}$ in Fig. 3.3 (bottom) while the corresponding normalized quadrupole strengths $\left(K 1=\frac{1}{B \rho} \frac{\partial B}{\partial z}\right.$ in $\left.m^{-2}\right)$ of focusing, defocusing and triplet focusing quadrupole families are plotted in Fig. 3.3 (top). These quadrupole settings are those which correspond to the "set values" of currents for the control system of the quadruple power supplies of SIS18 thereby meaning they are the ideal settings. In reality, the quadrupole strengths can be different from these values e.g. due to the quadrupole gradient errors. One experimental evidence of such errors is that the quadrupole settings of all three families are desired to vary in a way that the coherent tune remains constant throughout the ramp for both planes, but a tune shift is measured experimentally as shown in Fig. 3.5. On the other hand, the net magnitude of spatial model mismatch between injection and extraction remains almost same for all cycles as evident from Fig. 5.4 where the calculated highest singular values of the ORMs using MAD-X for the two different dipole ramps of $5 \mathrm{~T} / \mathrm{s}$ and $10 \mathrm{~T} / \mathrm{s}$ are plotted for comparison, for the ideal case. Moreover, one can see that the ORM variation in horizontal plane is more prominent $(\approx 45 \%$ of initial value $)$ than that in vertical plane $(\approx$ $6 \%)$ for SIS18.

In order to check the possibility of using a fixed-ORM based COFB system over the ramp, the fast dipole ramp of $10 \mathrm{~T} / \mathrm{s}$ (for ideal quadrupole settings) was selected and the spectral radius $\rho(\mathbf{M})$ between the ORMs $\mathbf{R}(t)$ at each time step of the ramp and the nominal ORM $\mathbf{R}_{\Theta}$ corresponding to the injection settings is defined as,

$$
\rho(\mathbf{M}(t))=\rho\left(\mathbf{I}-\mathbf{R}(t) \mathbf{R}_{\Theta}^{+}\right)
$$



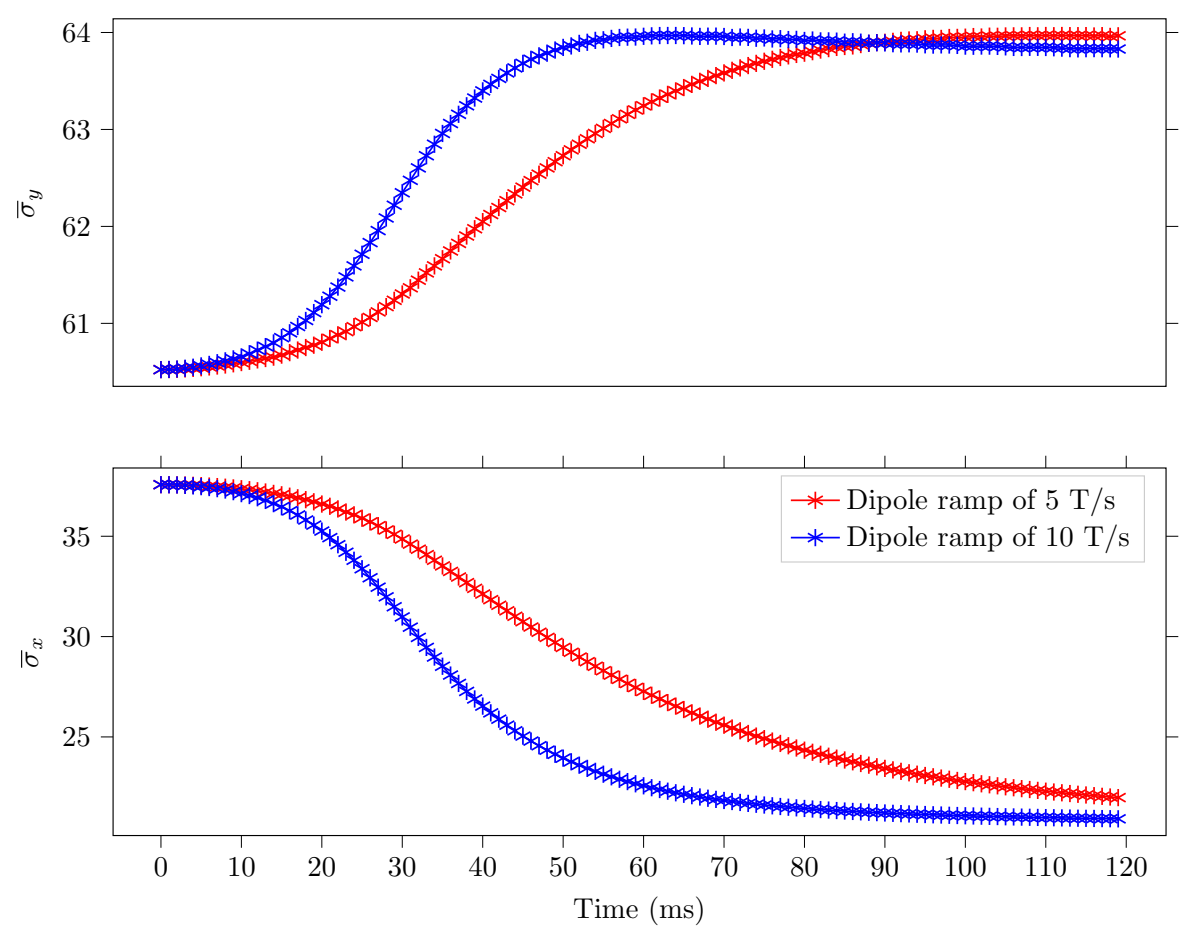

Figure 5.4 The variation of the highest singular values of the ORMs in both transverse planes, over the ramps of dipole graidents of $5 \mathrm{~T} / \mathrm{s}$ and $10 \mathrm{~T} / \mathrm{s}$.

The result of Eq. 5.22 is plotted in Fig. 5.5 for both planes (red for $x$-plane and black for $y$-plane). Furthermore, the correction ratio $\delta_{1}=\frac{\mathbf{r}_{1, \mathrm{RMS}}}{\mathbf{w}_{0, \mathrm{RMS}}}$ is also simulated for 1000 random perturbed orbits at each time step of the ramp by the following way: MAD-X is used for the generation of 1000 perturbed closed orbits at each time step of the ramp using the random combinations of transverse misalignment of quadruples with Gaussian probability distribution ( $\sigma=0.3 \mathrm{~mm}$ cut at $3 \sigma \approx 1 \mathrm{~mm}$ ). As a result the RMS values of the perturbed orbits also have a Gaussian distribution with mean $=12.5 \mathrm{~mm}$ and $\sigma=7.5 \mathrm{~mm}$. Corrector settings are calculated using all the singular values of $\mathbf{R}_{\Theta}$ for each perturbed orbit. The resultant correction ratios also have a Gaussian distribution but with a significantly smaller standard deviation and are also plotted in Fig. 5.5 as a function of time (blue for $x$-plane and green for $y$-plane). The dots in the plots comprises of the mean RMS of the Gaussian distribution of correction ratios while the error bar represent the standard deviation.

In the comparison of correction ratio to the $\rho(\mathbf{M}(t))$, one can see that the mean value of the first iteration residual increases in a direct proportionality to the $\rho(\mathbf{M})$ (according to inequality 5.19) with the increase of model mismatch in both planes, confirming that $\rho(\mathbf{M})$ is a practical figure of merit for quantifying the spatial model mismatch. Anyhow, a few orbits in $x$-plane have correction ratio larger than $\rho(\mathbf{M})$ (at the edge of standard deviation) which is a consequence of slight deviation from circulant symmetry in this plane while a pure circulant symmetry exists in the $y$-plane. The correction ratio is further simulated in $x$-plane over successive iterations of orbit correction as $\delta_{k}$ for a fixed model mismatch at $80 \mathrm{~ms}$ of the ramp where $\rho(\mathbf{M})=0.544$. The resultant $\delta_{k}$ is plotted in Fig. 5.6 for the first 7 iterations 


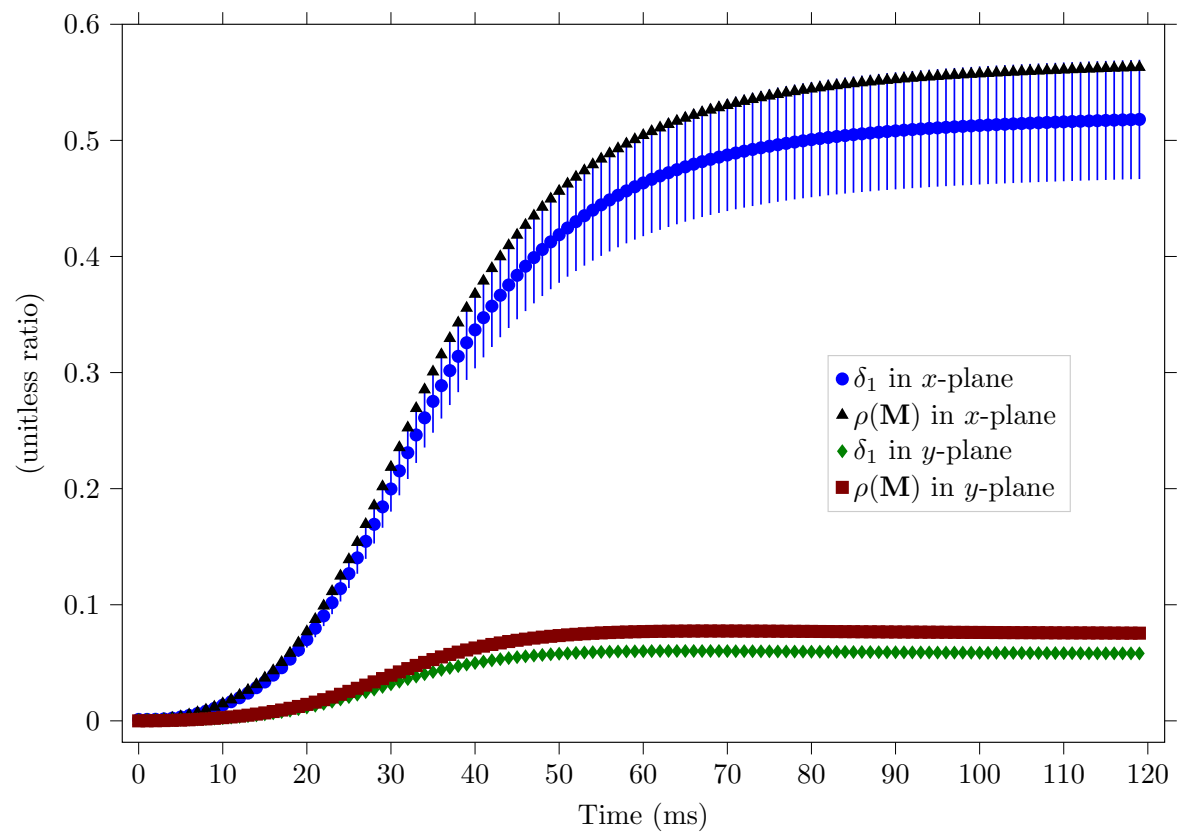

Figure 5.5 The simulated correction ratio $\delta_{1}=\frac{\mathbf{r}_{1, \mathrm{RMS}}}{\mathbf{w}_{0, \mathrm{RMS}}}$ and $\rho(\mathbf{M}(t))$ over a ramp of $10 \mathrm{~T} / \mathrm{s}$ for both planes.

after which the residual reaches to the noise level. One can see that the correction ratio of all simulated orbits is less than $\rho(\mathbf{M})$ asymptotically as predicted by inequality 5.20 for non-circulant ORMs. Moreover, the simulations suggest that the SIS18 COFB should never be unstable due to on-ramp model mismatch in the slow regime for the ideal quadrupole settings, as the maximum $\rho(\mathbf{M})$ is much less than 1 in any case. The only difference is that more ORMs will be needed to be updated in the $x$-plane in comparison to the $y$-plane, to achieve a comparable orbit correction in both planes.

As mentioned before, the tune shifts $\left(\Delta Q_{y} \approx 0.01\right.$ and $\left.\Delta Q_{x} \approx 0.02\right)$ are also observed experimentally which can be regarded as a non-systematic model mismatch. Tune shifts of comparable magnitudes have also been reported for electron beams during fast ramps e.g. at ELSA [82]. The exact reasons for such a tune shift is not trivial to determine because there may be many factors inter-playing together during the ramp e.g. output current of the power supplies not following the control curve, errors in the calibration of current to magnetic field of the magnets and eddy currents in the vacuum chambers or magnets. All these effects can result into the quadrupole field gradient errors during the ramp and consequently can affect the tune. The eddy currents in magnet cores are thought to be the primary cause of quadrupole gradient errors. Therefore the on-ramp tune-shift is simulated by application of low pass filtering to the ideal quadrupole strengths. Two low pass $1^{\text {st }}$ order butterworth type filters of cut-off frequencies $50 \mathrm{~Hz}$ and $35 \mathrm{~Hz}$ were applied to the quadrupole strengths in order to produce the vertical tune shift of $\Delta Q_{y, \max }=0.010$ and $\Delta Q_{y, \max }=0.020$ and the horizontal tune shift of $\Delta Q_{x, \max }=0.018$ and $\Delta Q_{x, \max }=0.032$, as shown in Fig. 5.7. The tune corresponding to ideal quadrupole strengths is also plotted as a reference (green). 


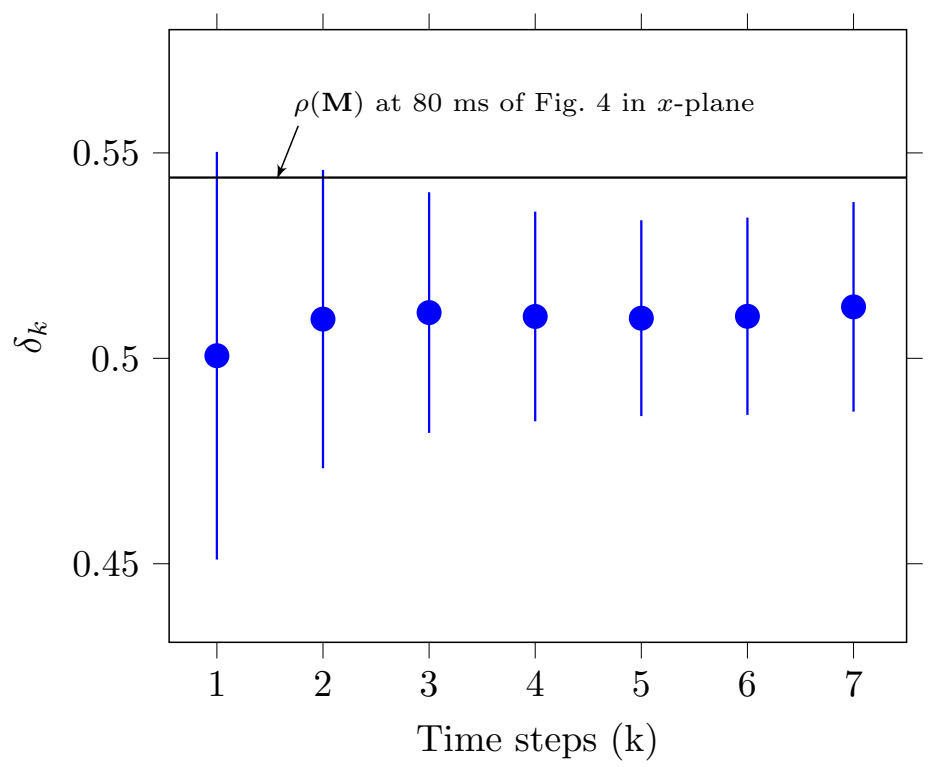

Figure 5.6 Correction ratio $\delta_{k}=\frac{\mathbf{r}_{k, \mathrm{RMS}}}{\mathbf{r}_{k-1, \mathrm{RMS}}}$ over subsequent iterations in agreement with Gelfand's formula.
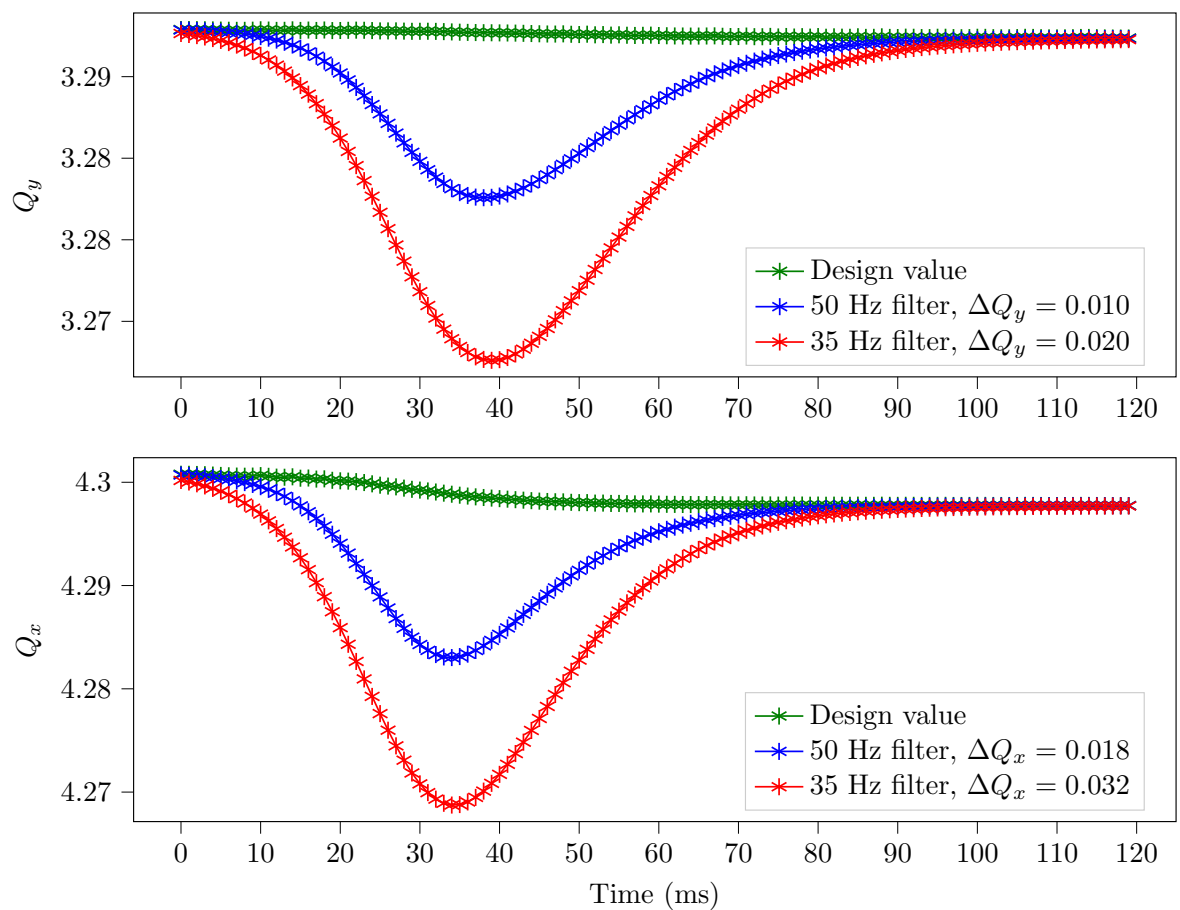

Figure 5.7 Simulated tune shifts over the ramp of dipole gradient of $10 \mathrm{~T} / \mathrm{s}$.

The contribution of such tunes shifts in the $\rho(\mathbf{M})$ is depicted in Fig. 5.8. Non-systematic tune shifts act as an additional model mismatch on top of that produced by the systematic model mismatch. However, the effect of tune shift is more dominant in vertical plane than in 


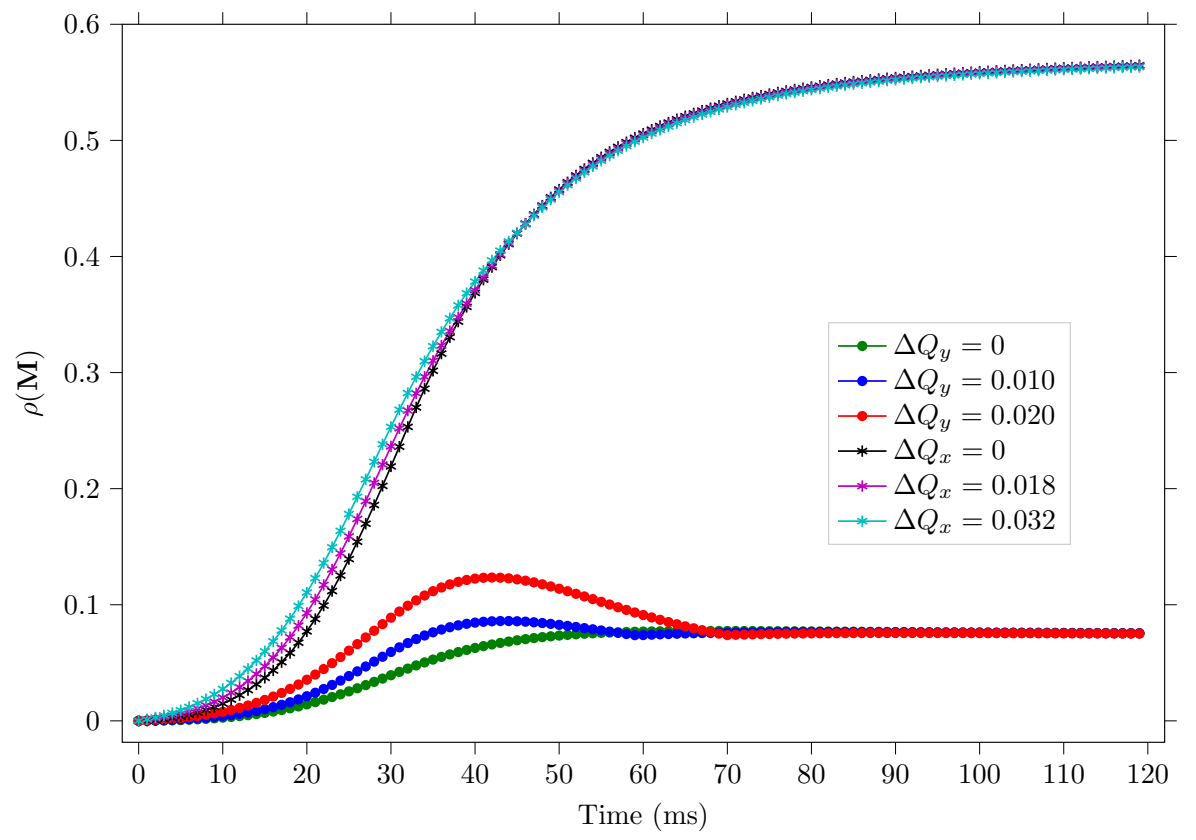

Figure $5.8 \rho(\mathbf{M})$ for both transverse planes over the ramp of dipole gradient of 10 $\mathrm{T} / \mathrm{s}$ for ideal quadrupole strengths as well as filtered for the tune variations.

horizontal plane.

\subsubsection{Intensity dependent tune shift}

The intensity-dependent tune shift can be modelled as the image charge effect of the vacuum chambers around the beam. Image charges of opposite sign pull the beam outward like a defocusing force causing a decrease in coherent tune. The image charge force is a non-linear function of the beam's transverse position [85] depending upon the boundary but can be linearized for small oscillations and for simple geometries (circular or elliptical) as performed in 86 and given as,

$$
F_{y}^{\text {image }} \propto y
$$

where $F_{y}^{\text {image }}$ is the defocusing force in the y-direction.

In order to simulate the image charge effect in MAD-X, all the drift regions of SIS18 were replaced with weak defocusing quadrupoles in $y$-plane and the same quadrupole strength was added to the strengths of already present quadrupole families resulting in a weak defocusing force throughout the synchrotron. As a result, we calculate the $\rho(\mathbf{M})$ for tune shift defined as,

$$
\rho(\mathbf{M}(\Delta Q))=\rho\left(\mathbf{I}-\mathbf{R}(\Delta Q) \mathbf{R}_{\Theta}^{+}\right)
$$

where $\mathbf{R}(\Delta Q)$ corresponding to ORM with image charge tune shifts for the vertical plane of SIS18. The corresponding $\bar{\sigma}\left(\Delta_{R}\right)$ (discussed in Eq. 5.16) quantifies the relative deviation of the singular values corresponding to the dominant (tune) modes of $\mathbf{R}_{\Theta}$ and $\mathbf{R}$. This implies that the allowed uncertainty in the ORM for a stable COFB will depend upon the initial 


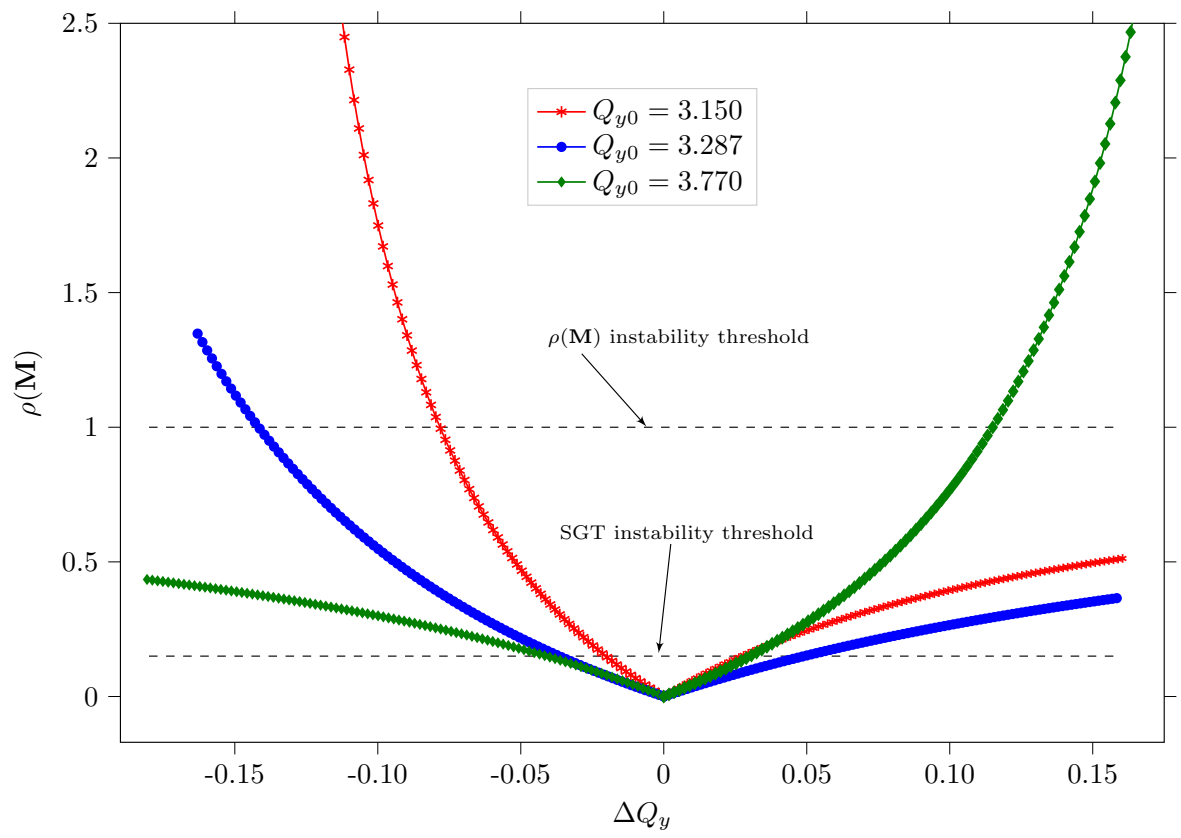

Figure $5.9 \rho\left(\mathbf{M}\left(\Delta Q_{y}\right)\right)$ for image charge based tune shift for three different initial vertical tunes. SGT means small gain theorem.

value of the tune and the direction of the tune shift towards or away from the integer value. This is due to the fact that the singular values have different growth rate when the tune moves towards (fast increase of spectral radius) or away (slow increase of spectral radius) from the integer value. In order to confirm this, three working points $Q_{y 0}=3.287,3.150$ and 3.770 are selected to calculate the ORMs corresponding to the zero tune shifts. The tune of the machine is then changed in both directions for all three cases and $\rho(\mathbf{M})$ is plotted in Fig. 5.9 . The stability margins defined by the small gain theorem is also highlighted along with that of spectral radius condition, for comparison. One can see that for an initial working point of 3.15 (red), the instability threshold is reached with a negative tune shift of only -0.0782 while for the $Q_{y 0}=3.77$ even a negative tune shift of -0.180 makes $\rho(\mathbf{M})=0.43$ (far from instability threshold of 1 ). On the other hand, $\rho(\mathbf{M})$ crosses the instability threshold for a small positive tune shift of +0.116 for $Q_{y 0}=3.77$.

For a further comparison, a fixed tune shift of -0.079 (for which red curve crosses 1 for the value of $\rho(\mathbf{M})$ in Fig. 5.9 ) is selected for all three above mentioned initial tunes and the orbit correction over many time steps is simulated using MAD-X for the slow regime of SIS18. The corrector settings for each time step $k$ are calculated as $\boldsymbol{\Theta}_{k}=\boldsymbol{\Theta}_{k-1}-\mathbf{R}_{\Theta}^{+} \mathbf{y}_{k}$ where $\mathbf{R}_{\Theta}^{+}$ is the pseudo-inverse of ORM corresponding to initial tune while the actual machine tune in MAD-X is $Q_{y 0}-0.079$, for each case. The RMS values of the resultant closed orbits over each time step are plotted in Fig. 5.10 where $k=0$ corresponds to the initial perturbed orbits.

One can see that the orbit correction is unstable for the case of initial tune $Q_{y 0}=3.15$ in agreement with $\rho(\mathbf{M})>1$ for $\Delta Q=-0.079$ and $\rho(\mathbf{M})=1.037$ as evident in Fig. 5.9. For the other two cases, where the $\rho(\mathbf{M})$ is less than 1 , the closed orbit necessarily converges 


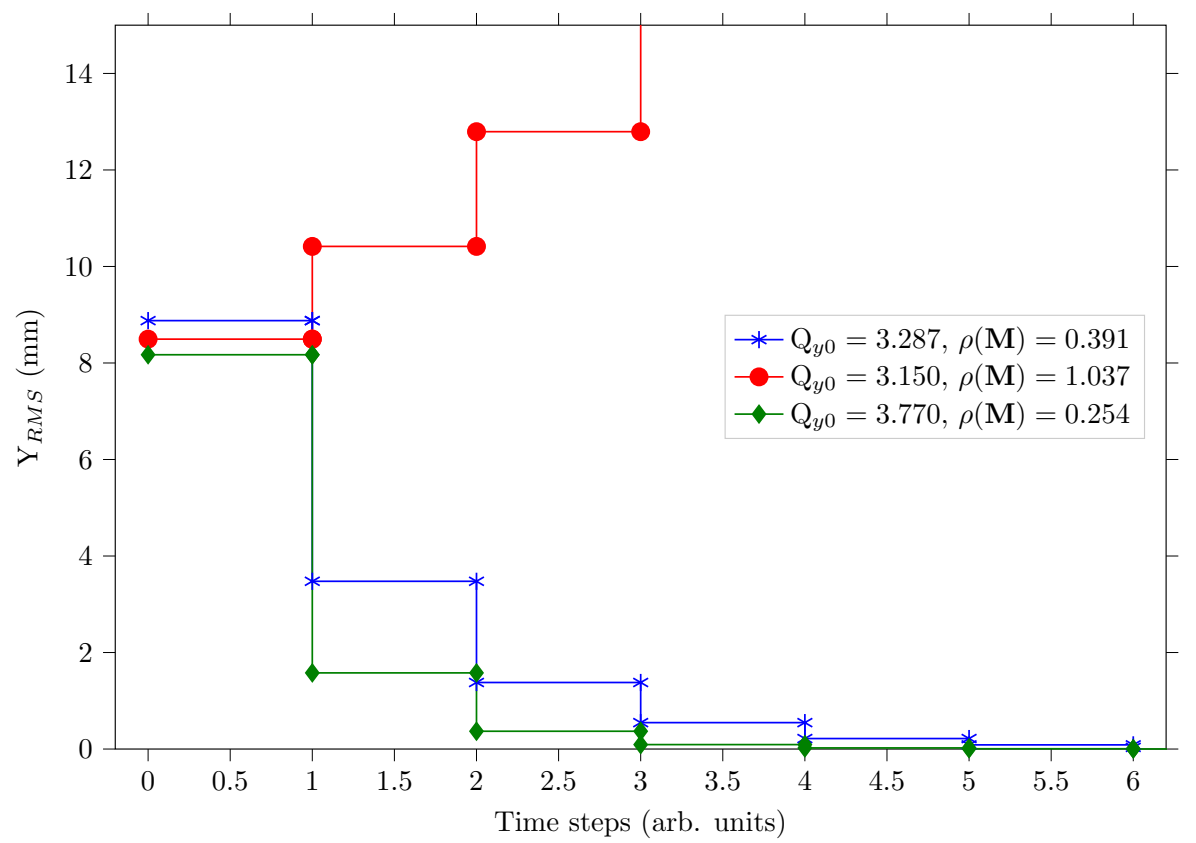

Figure 5.10 Simulated closed orbit RMS over successive time steps of correction in MAD-X for a fixed $\Delta Q=-0.079$ in the slow regime of SIS18 for three initial machine tunes.

to zero even if the stability margin defined by the small gain theorem (i.e. $\rho(\mathbf{M})=0.15$ ) is already crossed. The required number of time steps can be seen to be proportional to the magnitude of $\rho(\mathbf{M})$. These calculations confirm that it is not only the magnitude of the tune shift that defines the instability threshold of the COFB system but also the direction towards or away from the integer value. Tune shifts away from the integer value ensure the COFB system stability. It is important to mention that COFB system instabilities due to spatial model mismatch are discussed for scenerios when the machine is itself stable against tune resonances.

\subsubsection{Beta beating}

Beta beating is another source of model error that can vary the ORM of the machine. It is the deviation of the beta function from its periodic values caused by the localized quadrupole field errors in a synchrotron and is defined for random field errors $\Delta K_{i}$ as [15],

$$
\begin{aligned}
\Delta \beta\left(l_{0}\right)= & \frac{\beta_{0}}{2 \sin (2 \pi Q)} \sum_{i} \int_{0}^{L} \Delta K_{i} \beta_{i}(l) \\
& \cdot \cos \left(2\left(\mu(l)-\mu_{0}\right)-2 \pi Q\right) d l
\end{aligned}
$$



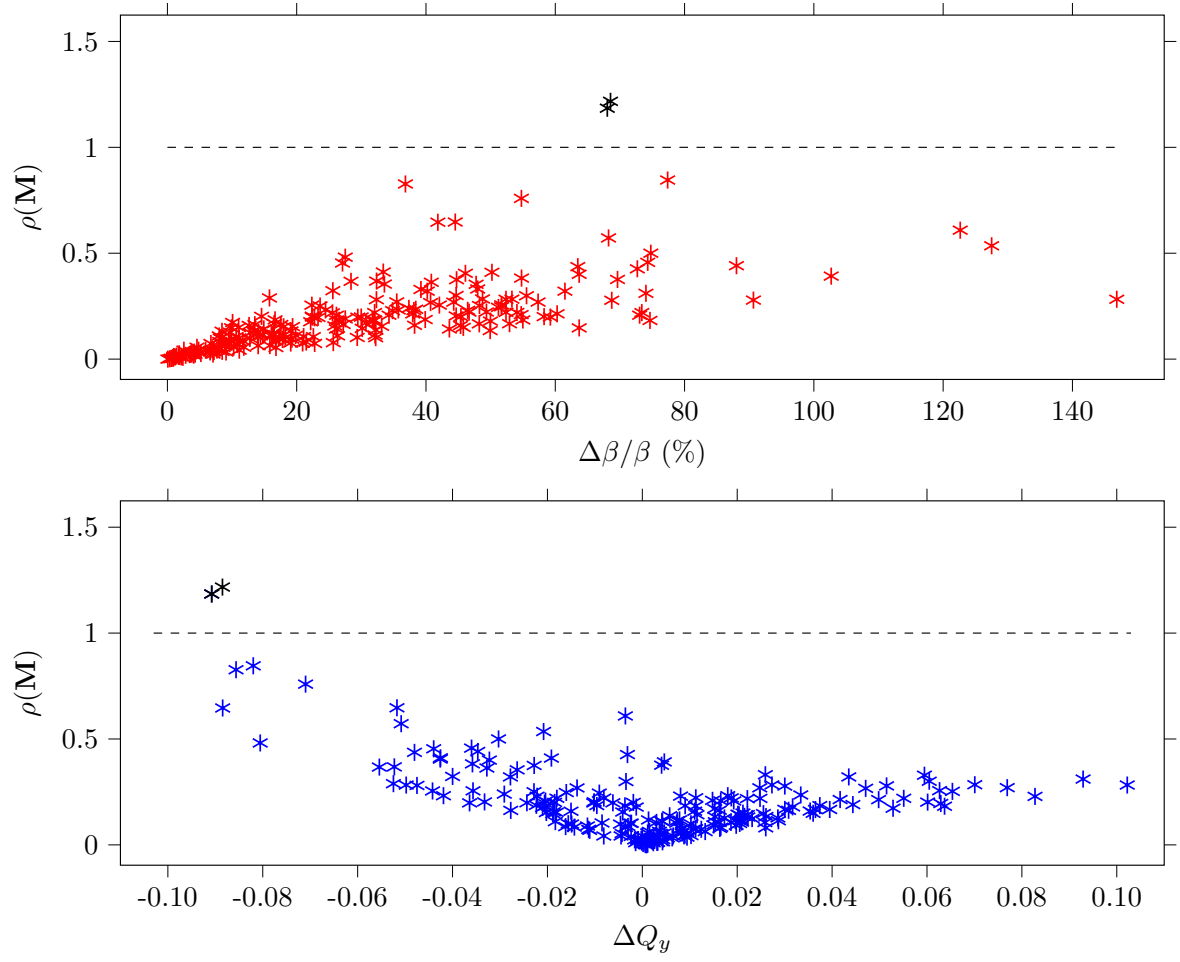

Figure $5.11 \rho\left(\mathbf{M}\left(\frac{\Delta \beta}{\beta}\right)\right)$ for beta beating produced by randomly distributed field errors for an initial vertical tune $Q_{y 0}=3.287$. Top: Versus beta beating. Bottom: Versus tune shift.

while the corresponding tune variation is

$$
\Delta Q=\frac{1}{4 \pi} \sum_{i} \int_{0}^{L} \Delta K_{i} \beta(l) d l
$$

Here $L$ is the length of the quadrupole and $l$ is the longitudinal coordinate of the synchrotron. The other parameters are already defined in chapter. 2. Figure 5.11(top) shows the simulations for $y$-plane of $\rho\left(\mathbf{M}\left(\frac{\Delta \beta}{\beta}\right)\right)$ using $\mathbf{R}_{\Theta}$ corresponding to zero beta beating and $Q_{y 0}=3.287$ for a range of beta beating up to $125 \%$ generated by random distribution of quadrupole field errors in MAD-X. One can see that the orbit correction with an improper model, can lead to instability $(\rho(\mathbf{M})>1)$ even for $67 \%$ of peak-peak beta beating (black stars in Fig. 5.11) and on the other hand, can converge $(\rho(\mathbf{M})<1)$ even for more than $100 \%$ of peak-peak beta beating. This observation is explained in Fig. 5.11 (bottom) where $\rho(\mathbf{M})$ is plotted as a function of tune shift. The $\rho(\mathbf{M})$ is larger than 1 only for negative tune shifts. The reason is that the negative tune shift takes the working point of the machine (in MAD-X) closer to the integer value in this case, as also discussed in the previous subsection. This assertion is confirmed by adjusting the unperturbed vertical tune at $Q_{y 0}=3.77$ so that for positive tune shifts, the working point moves towards the integer value (contrary to previous case). Figure 5.12 shows the corresponding $\rho(\mathbf{M})$ as function of both beta beating and tune shift. 

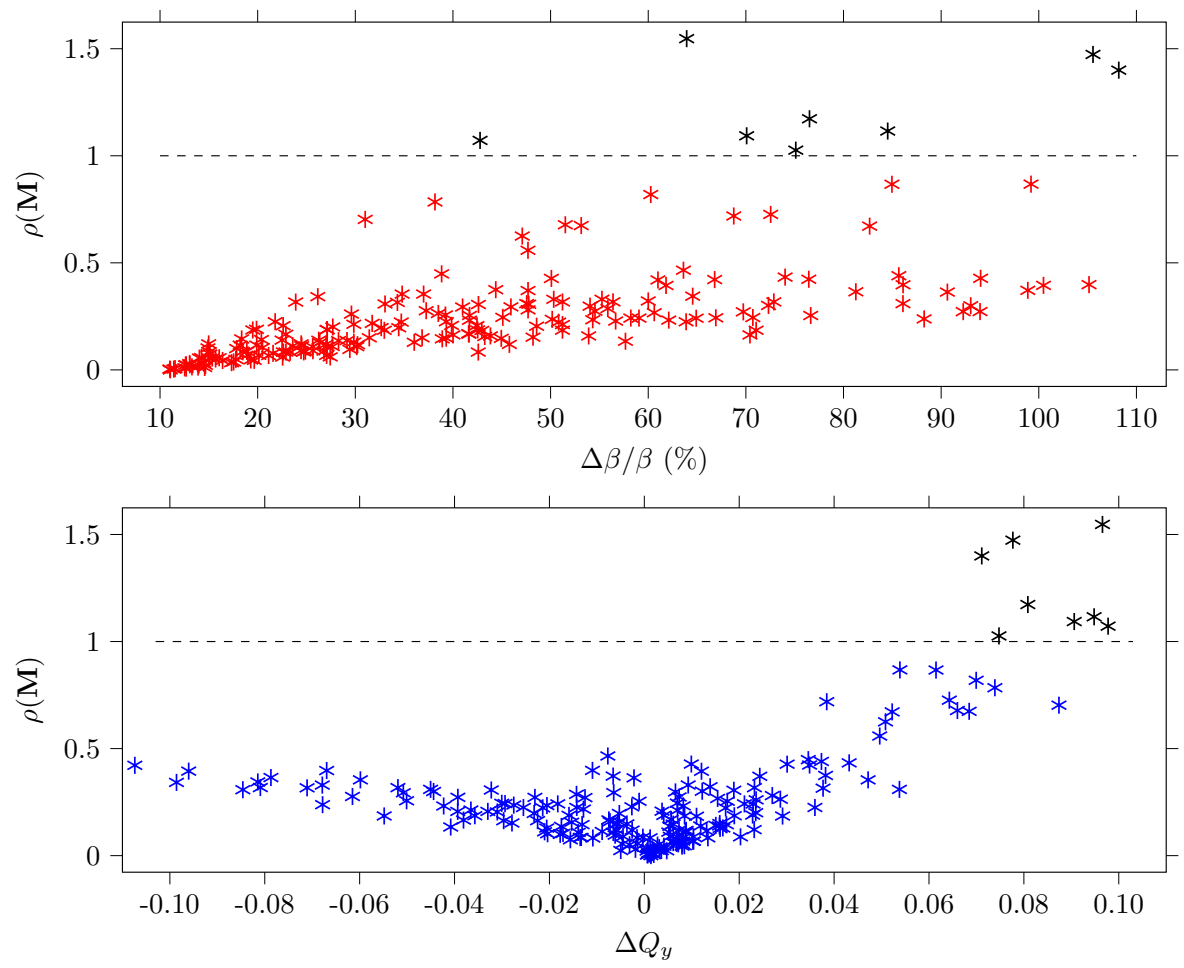

Figure $5.12 \rho\left(\mathbf{M}\left(\frac{\Delta \beta}{\beta}\right)\right)$ for beta beating produced by randomly distributed field errors for an initial vertical tune $Q_{y 0}=3.770$. Top: Versus beta beating. Bottom: Versus tune shift.

The behavior is similar to the previous case but mirrored with respect to tune shift. Thus we conclude, that the magnitude of beta beating itself does not play a large role in context of orbit correction (also observed at other facilities e.g. BNL 87]) but only when accompanied by the tune shift towards the integer value, beta beating can lead to orbit instability.

\subsection{Experimental verification at COSY FZJ}

Some of the conclusions drawn in sec. 5.5 were experimentally verified at the COFB system of proton Cooler Synchrotron at Forschungzentrum Jülich (FZJ). The correction time for the operational COFB system is $T_{s}=2 s$ and therefore, it can be regarded as a slow feedback system with temporal characteristics of the power supplies and BPMs ignored. The on-ramp ORM variation and artificially introduced tune shift were used as the sources of model mismatch. By default, the singular values of the ORM in the controller having magnitude below the $10 \%$ of the highest singular value were truncated. 


\subsubsection{Vertical tune variation}

As concluded in sec. 5.5.2, it is not only the magnitude of the tune shift but its direction towards or away from integer tune that defines the instability threshold of the COFB system. In order to verify this experimentally, the machine settings of COSY corresponding to the vertical tune of $Q_{y 0}=3.62$ were fixed while the ORMs were updated in the feedback system for a range of tune values from 3.53 to 4.16. The resultant measured correction

ratio $\left(\delta_{1}=\frac{\mathbf{r}_{1, \mathrm{RMS}}}{\mathbf{y}_{0, \mathrm{RMS}}}\right.$, replacing $\mathbf{w}$ with $\mathbf{y}$ for vertical plane in Eq. 5.18) is plotted in Fig. 5.13 (top). One can see that the correction ratio increases as the tune moves towards the integer value. Figure 5.13 (bottom) shows the corresponding calculated $\rho(\mathbf{M})$ in blue which is largely different from measured correction ratio primarily around $\Delta Q_{y}=0$ showing an inherent model mismatch between the actual (unknown) model (let us call it $\mathbf{R}_{X}$ ) and the best known (measured) model $\mathbf{R}$ of the machine. Since $\mathbf{R}$ is the known model in this case, we can condition it before pseudo-inversion by SVD so that $\mathbf{R R}^{+}=\mathbf{I}$. Such a model mismatch can be associated to the corrector calibration errors resulting in a multiplicative uncertainty such that $\mathbf{R}_{X}=\left(\mathbf{I}+\Delta_{\mathbf{R}}\right) \mathbf{R}$. The effect of such an inherent model mismatch can be excluded from simulations using the measured correction ratio for $\Delta Q_{y}=0$. Let us call it $\mathbf{r}_{1}^{\prime}$

$$
\begin{aligned}
\mathbf{r}_{1}^{\prime} & =\left(\mathbf{I}-\mathbf{R}_{X} \mathbf{R}^{+}\right) \mathbf{y}_{0} \\
\mathbf{r}_{1}^{\prime} & =-\Delta_{\mathbf{R}} \mathbf{y}_{0}
\end{aligned}
$$

The effect of intentionally varied model $\mathbf{R}_{\Theta}$ (controller model) on the residual $\mathbf{r}_{1}$ with respect to the actual ORM $\mathbf{R}_{X}$ can be written as

$$
\begin{aligned}
& \mathbf{r}_{1}=\left(\mathbf{I}-\mathbf{R}_{X} \mathbf{R}_{\Theta}^{+}\right) \mathbf{y}_{0} \\
& \mathbf{r}_{1}=\left(\mathbf{M}-\Delta_{\mathbf{R}}(1-\mathbf{M})\right) \mathbf{y}_{0}
\end{aligned}
$$

where $\mathbf{M}=\left(\mathbf{I}-\mathbf{R R}_{\Theta}^{+}\right)$. Using inequality 5.19 and the triangular inequalities of matrix norm $(\|\mathbf{A}+\mathbf{B}\| \leq\|\mathbf{A}\|+\|\mathbf{B}\|$ and $\|\mathbf{A B}\| \leq\|\mathbf{A}\| \cdot\|\mathbf{B}\|)$ the correction ratio can be deduced from Eq. 5.28 as

$$
\frac{\mathbf{r}_{1, \mathrm{RMS}}}{\mathbf{y}_{0, \mathrm{RMS}}} \leq \rho(\mathbf{M})+\frac{\mathbf{r}_{1, \mathrm{RMS}}^{\prime}}{\mathbf{y}_{0, \mathrm{RMS}}}(1-\rho(\mathbf{M}))
$$

The right-hand side of non-strict inequality 5.29 is plotted in red in Fig. 5.13 (bottom) in order to match the experimentally measured correction ratio. Figure 5.14 shows the measured closed orbit RMS over a number of time steps for specific tune values. Even up to a very large tune shift $\left(Q_{y}=3.97\right)$, the COFB system is stable since $\rho(\mathbf{M})$ is still less than 1 (Fig. 5.13 bottom). For the tune shifts $Q_{y}>4$, the $\rho(\mathbf{M})>1$ and the COFB system gets unstable as seen by the increasing residual over successive time steps in Fig. 5.14 resulting in the beam loss.

\subsubsection{On-ramp ORM variation}

At COSY, proton beam was accelerated from $45 \mathrm{MeV}$ to $283 \mathrm{MeV}$ and the orbit corrections were performed in both planes at injection, mid-ramp $(122 \mathrm{MeV})$ and extraction energies using only the ORM corresponding to the extraction settings. The measured temporal evolution of 

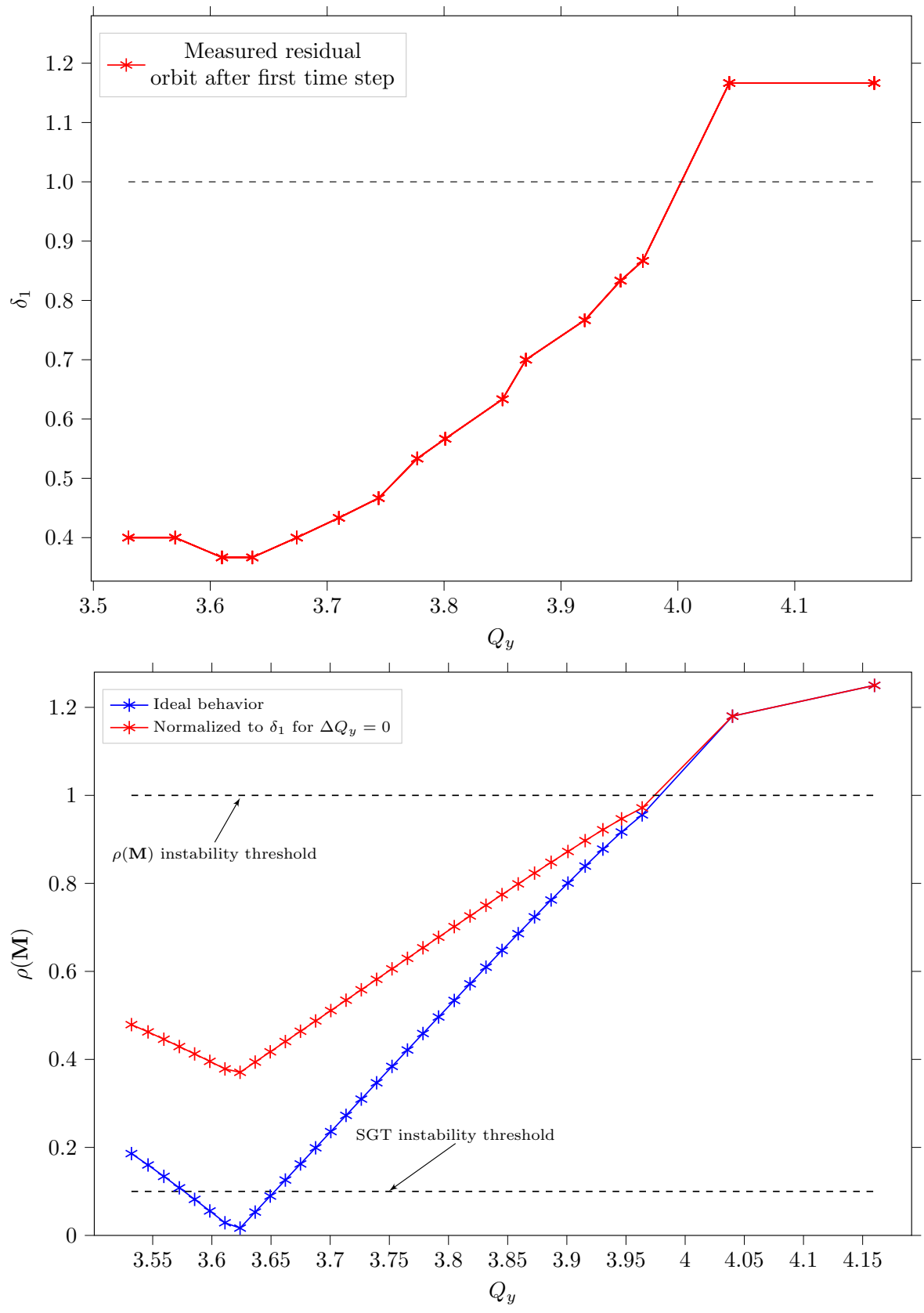

Figure 5.13 Measured ratio $\delta_{1}=\frac{\mathbf{r}_{1, \mathrm{RMS}}}{\mathbf{y}_{0, \mathrm{RMS}}}$ in the vertical plane of COSY for the fixed machine tune of $Q_{y 0}=3.62$ while varying the ORM in the feedback system corresponding to a tune range of 3.53 to 4.16 (top). Calculated $\rho(\mathbf{M})$ for vertical plane (bottom). SGT means small gain theorem. For ideal and realistic case please see the text.

the closed orbit RMS is plotted as the function of time steps in Fig. 5.15 (top) for $x$-plane and (bottom) for $y$-plane for all three machine settings. One can see that the residual after 


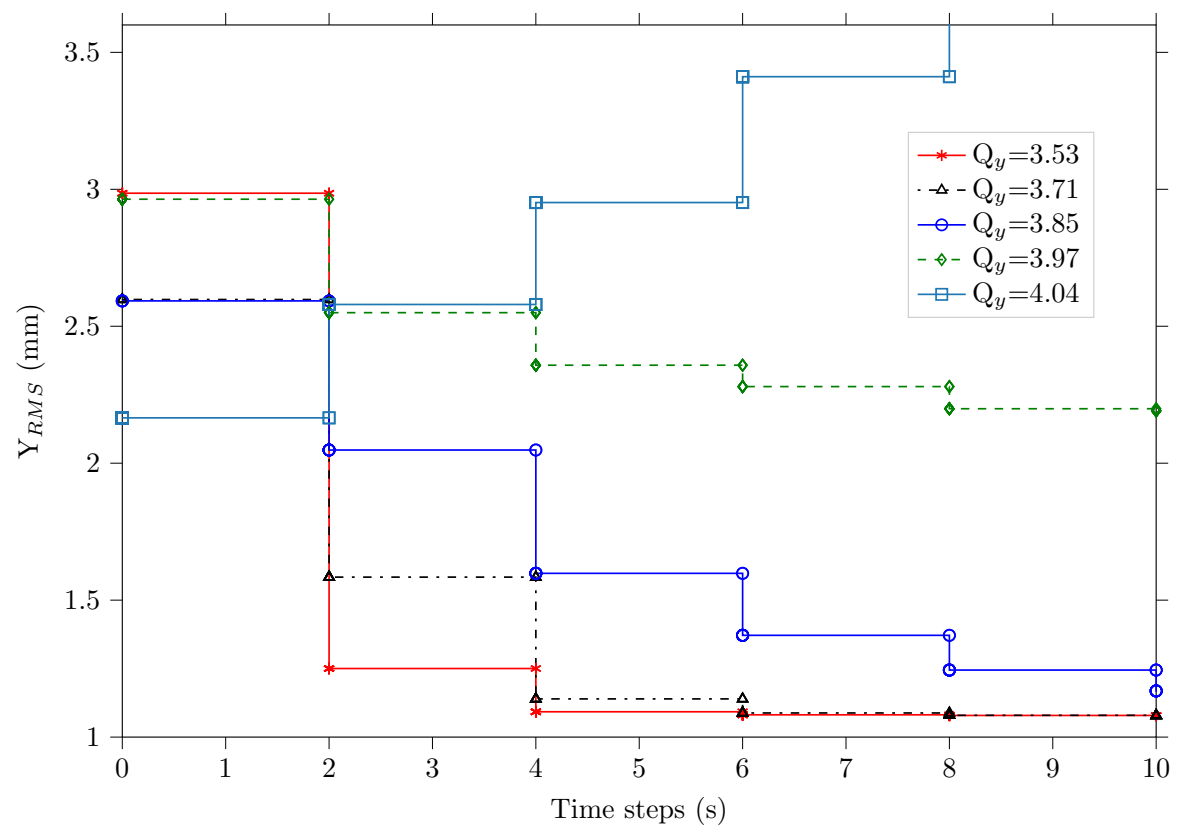

Figure 5.14 Measured RMS of the closed orbit in $y$-plane of COSY synchrotron for different ORMs used for the corrector settings calculation while keeping the machine settings fixed for a tune of 3.62 .

the first time step as well as the number of correction steps required for the orbit convergence increase with the increased model mismatch. In all three cases, closed orbit converges to a minimum, confirming that the COFB system stability is ensured if the correction ratio is less than 1 , in agreement with the spectral radius stability condition of inequality 5.12 . Two observations can be made concerning the residual orbit. 1) A non-zero residual orbit remains after the first correction step even at extraction due to the inherent model mismatch, as discussed in the previous subsection. 2) The residual orbit over successive correction steps converges to different values for the injection, mid ramp and extraction. This is most likely due to the change in orbit pattern and a different coupling to the truncated SVD modes in $\mathbf{R}_{\ominus}$ in each of those cases.

\subsection{Spatial model mismatch in the fast temporal regime}

In deriving the stability condition for the slow feedback regime i.e. inequality 5.12 , the temporal parts of the system and the controller were ignored. Here, the treatment is extended to the fast regime where the system dynamics plays a role in the performance of the COFB system, in the generic form. The analysis is made in the discrete $z$-domain but can also be equally valid for the Laplace domain if the sampling frequency is at least an order of magnitude higher than the required bandwidth of the feedback system.

First of all, the transition of parameters of interest from slow regime to the fast regime can be made intuitively as: the notion of the required number of discrete time steps for orbit 

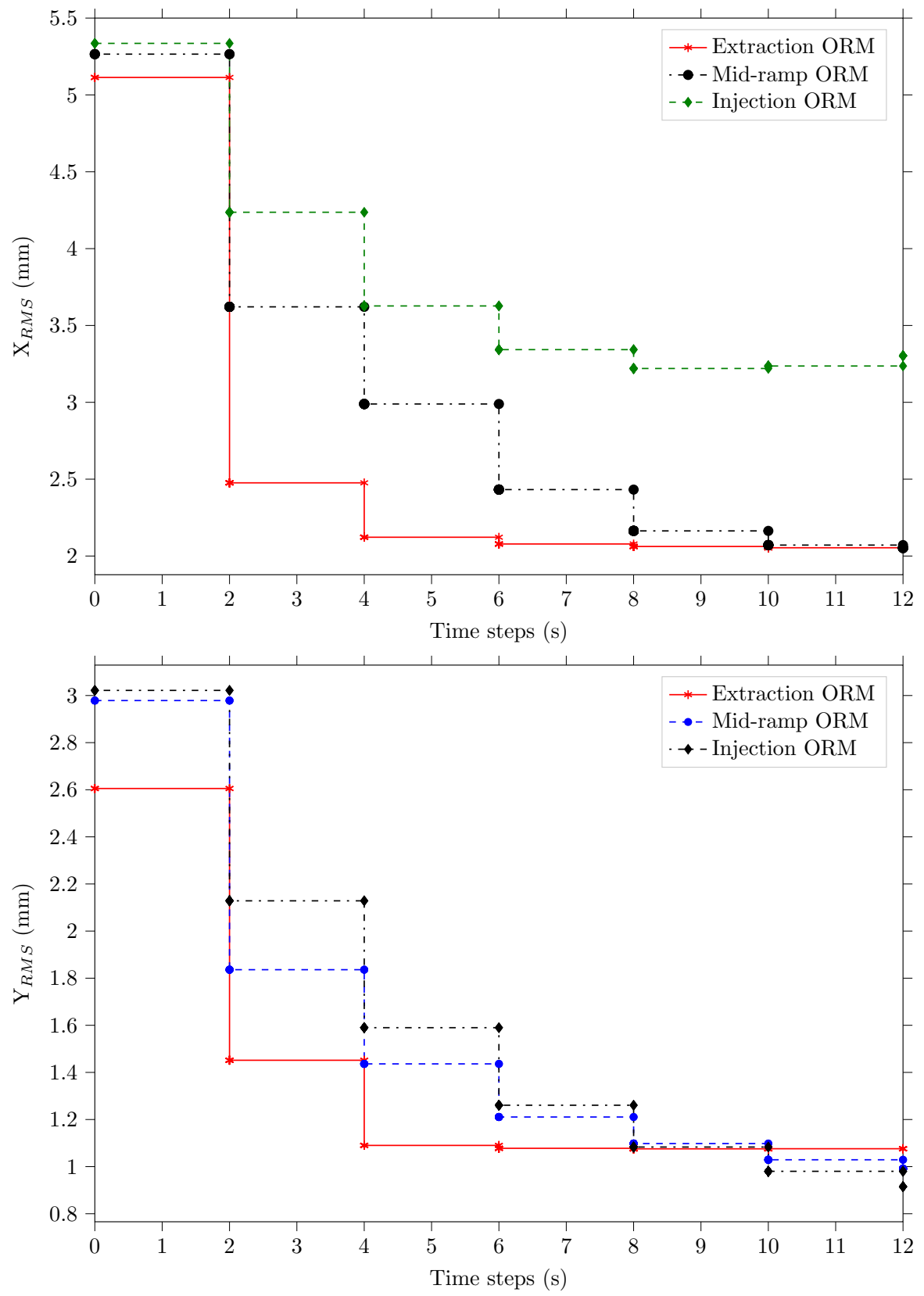

Figure 5.15 Measured RMS of the closed orbit in $x$-plane (top) and in $y$ - plane (bottom) for machine settings corresponding to injection, mid-ramp and extraction energy while using the ORM corresponding to extraction settings for the correctorsettings calculation.

convergence in slow regime can be exchanged with the closed-loop bandwidth for the fast regime. As larger the value of $\rho(\mathbf{M})$ is, as higher is the residual $r_{1}$ after first orbit correction. This results in more time steps or a longer time constant required for the convergence of 
the closed orbit. The longer time constant is equivalent to the reduction of the achievable bandwidth of the COFB system for the attenuation of the dynamic orbit errors.

For a quantitative analysis, following important transfer function matrices for the closed-loop shown in Fig. 5.1 can be written as,

$$
\begin{aligned}
\mathbf{L}(z) & =\mathbf{G}(z) \mathbf{K}(z) \\
\mathbf{S}(z) & =(\mathbf{I}+\mathbf{G}(z) \mathbf{K}(z))^{-1} \\
\mathbf{T}(z) & =(\mathbf{I}+\mathbf{G}(z) \mathbf{K}(z))^{-1} \mathbf{G}(z) \mathbf{K}(z) \\
& =(\mathbf{I}+\mathbf{L}(z))^{-1} \mathbf{L}(z)
\end{aligned}
$$

where $\mathbf{L}(z)$ is called the loop transfer function, $\mathbf{S}(z)$ is the sensitivity function from disturbance $\mathbf{d}(z)$ to the output $\mathbf{r}(z)$ and $\mathbf{T}(z)$ is the complementary transfer function from set value $\mathbf{w}_{r}$ to the output $\mathbf{r}(z)$.

In order to estimate the bandwidth of disturbance rejection, the sensitivity function $\mathbf{S}(z)$ is the right choice to be used such that the lowest frequency where $|\mathbf{S}(z)|$ has magnitude 1 is called sensitivity crossover frequency $f_{s c}$. Similarly, the positions of the poles of the loop transfer function $\mathbf{L}(z)$ and complementary sensitivity function $\mathbf{T}(z)$ on the complex plane predict the instability of the feedback system (for details see chapter 2).

For the COFB system of Fig. 5.1. the sensitivity function $\mathbf{S}(z)$ can also be written as,

$$
\begin{aligned}
\mathbf{S}(z) & =\left[\mathbf{I}+g(z) \mathbf{R} k(z) \mathbf{R}_{\Theta}^{+}\right]^{-1} \\
& =\left[\mathbf{I}+\mathbf{I} g(z) k(z)-\left(\mathbf{I}-\mathbf{R} \mathbf{R}_{\Theta}^{+}\right) g(z) k(z)\right]^{-1}
\end{aligned}
$$

One can see that the matrix $\mathbf{M}=\left(\mathbf{I}-\mathbf{R} \mathbf{R}_{\Theta}^{+}\right)$appears above in the temporal sensitivity function in order to affect the achievable bandwidth. Eq. 5.31 is a general equation of sensitivity function which can be solved for each mode of the ORM and for any controller $k(z)$. In order to find out the effect of spatial model mismatch on the disturbance rejection, the sensitivity function $S_{\rho}(z)$ corresponding to the highest eigenvalue of matrix $\mathbf{M}$ i.e. the spectral radius $\rho(\mathbf{M})$ can be defined by the replacement of $\mathbf{M}$ with $\rho(\mathbf{M})$ in Eq. 5.31 and by rearrangement as,

$$
S_{\rho}(z)=\frac{\frac{1}{1+g(z) k(z)}}{1-\rho(\mathbf{M}) \frac{g(z) k(z)}{1+g(z) k(z)}}
$$

where $g(z)$ and $k(z)$ are scalar functions. The above equation is actually an approximation of the MIMO system with a SISO system for $\rho(\mathbf{M})$, which carries the largest effect of model mismatch on the bandwidth, since all other eigenvalues will be smaller than $\rho(\mathbf{M})$.

For further analysis, the first order system model of Eq. 5.5 is considered as $g(z)=z^{-l} \frac{b_{0} z+b_{1}}{z-a_{1}}$ and a control law $k(z)$ can be calculated in a generic form using Internal Model Controller (IMC) methodology discussed in sec. 2.8.4 and is given as,

$$
k(z)=[g(z)]^{-1} \frac{Z(z)}{1-Z(z)}
$$

where $Z(z)=z^{-l} \frac{b_{0} z+b_{1}}{z-a_{1}}$ is a low pass filter. Using Eqs. 5.5 and 5.33 , the $\rho(\mathbf{M})$-dependent sensitivity function of Eq. 5.32 can be written as,

$$
S_{\rho}(z)=\frac{1-Z(z)}{1-\rho(\mathbf{M}) Z(z)}
$$


The corresponding loop transfer function $L_{\rho}(z)$ can be calculated from its corresponding relation to the sensitivity function from Eq. 5.30 as,

$$
L_{\rho}(z)=\frac{1-S_{\rho}(z)}{S_{\rho}(z)}
$$

Putting Eq. 5.34 in Eq. 5.35 yields

$$
\begin{aligned}
L_{\rho}(z) & =\frac{Z(z)(1-\rho(\mathbf{M}))}{1-Z(z)} \\
& =L_{(\rho=0)}(z)(1-\rho(\mathbf{M}) \\
& =g(z) k(z)(1-\rho(\mathbf{M}) \\
& =g(z) k_{\rho}(z)
\end{aligned}
$$

where $L_{(\rho=0)}(z)$ is the loop transfer function when the spatial models of system and controller cancel each other and can be calculated using $g(z)$ and $k(z)$ given in Eqs. 5.5 and 5.33 , respectively as,

$$
L_{(\rho=0)}(z)=g(z) k(z)=\frac{Z(z)}{1-Z(z)}
$$

Eqs. 5.36 gives a direct scaling of the controller with spatial model mismatch as a function of $\rho(\mathbf{M})$ as,

$$
k_{\rho}(z)=k(z)(1-\rho(\mathbf{M}))
$$

with reference to $k(z)$, the controller corresponding to $\rho(\mathbf{M})=0$.

The dependence of the complementary transfer function $T_{\rho}(z)$ on $\rho(\mathbf{M})$ can also be written in this case, using its relation with the loop transfer function given in Eq. 5.30 as,

$$
T_{\rho}(z)=\frac{L_{\rho}(z)}{1+L_{\rho}(z)}=\frac{Z(z)(1-\rho(\mathbf{M}))}{1-\rho(\mathbf{M}) Z(z)}
$$

\subsubsection{Temporal bandwidth}

In order to demonstrate the effect of spatial model mismatch on the disturbance rejection bandwidth of the feedback loop, the magnitude of the sensitivity function $S_{\rho}(z)$ defined in Eq. 5.34 is plotted in Fig. 5.16 for a range of $0<\rho(\mathbf{M})<1$ and for the following low pass filter,

$$
Z(z)=z^{-1} \frac{0.413}{z-0.586}
$$

calculated for the bandwidth of $850 \mathrm{~Hz}, \tau_{d}=100 \mu \mathrm{s}$ and $T_{s}=100 \mu \mathrm{s}$. One can see that there is no disturbance rejection for $\rho(\mathbf{M})=1$ as $\left|S_{\rho}(z)\right|=1$ for all frequencies. For $\rho(\mathbf{M})<1$, the sensitivity crossover frequency $f_{s c}$ decreases with the increase of $\rho(\mathbf{M})$ from 0 to 1 which is plotted in Fig. 5.17 (red) as a function of $\rho(\mathbf{M})$. It is evident that the spatial model mismatch has detrimental effect on the achievable bandwidth of the COFB system for the disturbance rejection.

The reduction of sensitivity crossover frequency also happens in the presence of pure delay in 


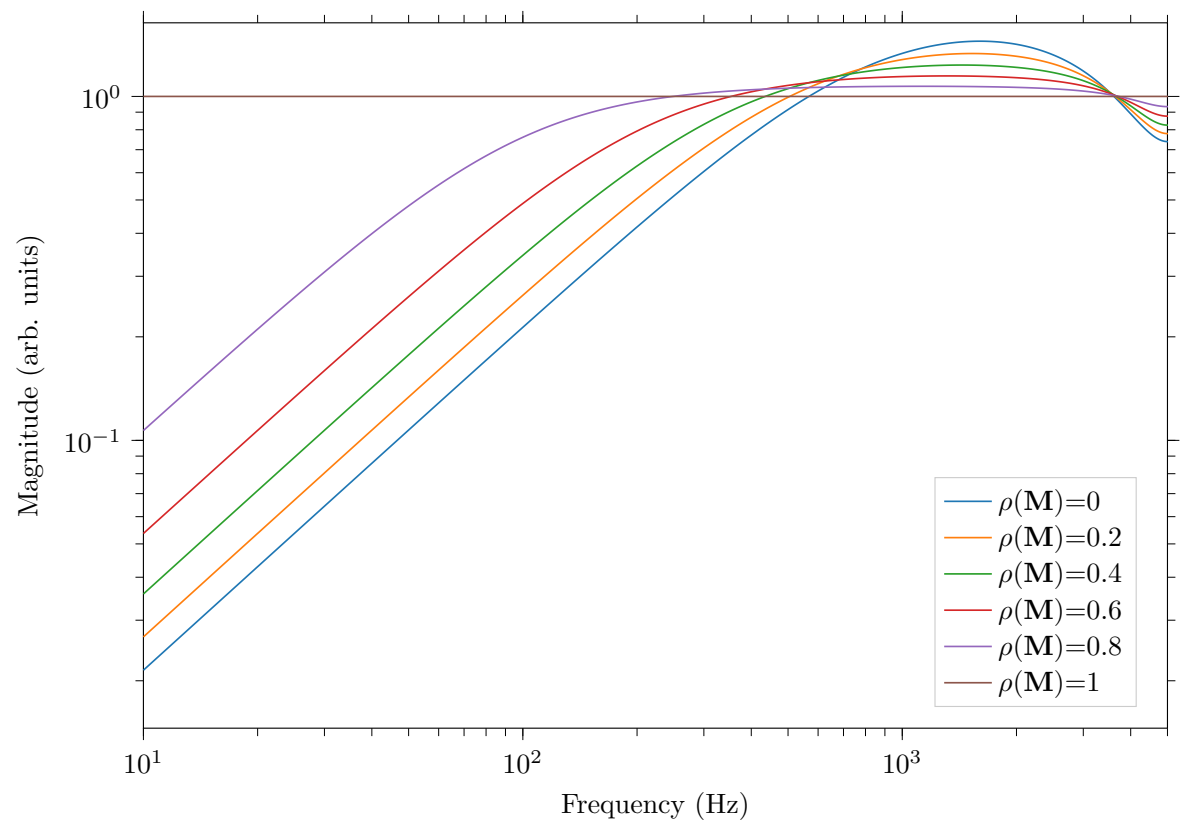

Figure 5.16 Sensitivity function of internal model controller (IMC) vs. frequency for various values of $\rho(\mathbf{M})$ between 0 and 1 .

the feedback loop (chapter 5 of [47]). For a comparison between the pure delay and spatial model mismatch, the sensitivity functions were also calculated (not shown) using the IMC methodology for $\rho(\mathbf{M})=0$ and for a range of pure delay $\tau_{d}$ from $100 \mu \mathrm{s}$ to $700 \mu \mathrm{s}$. $f_{s c}$ vs. $\tau_{d}$ curve is shown in blue in Fig. 5.17. Both pure delay and the spatial model mismatch limit the bandwidth for disturbance rejection but in a slightly different way. There is a weak reduction in bandwidth when $\rho(\mathbf{M})$ moves from 0 to 0.2 , and the drop is much sharper when $\rho(\mathbf{M})$ approaches unity, meaning closed-loop is more sensitive to spatial model mismatch near the instability region of $\rho(\mathbf{M})=1$. These results are very helpful to decide the required ORM update rate in the COFB controller for a variable optics machine e.g. for the on-ramp orbit correction in booster rings. For a given dynamical system and for the required bandwidth, one can calculate the allowed range of $\rho(\mathbf{M})$ using Eq. 5.34 which can be kept below a certain threshold by updating the $\mathbf{R}_{\Theta}^{+}$in the controller after appropriate time steps.

\subsubsection{Closed-loop stability}

Equation 5.36 suggests that for the fast regime of the orbit correction, $\rho(\mathbf{M})>1$ will change the sign of the controller gains making the positive feedback, resulting in the closed-loop instability. This is regardless of the dynamics of the system and the parameters of the controller and is consistent to the instability condition of slow regime given in inequality 5.12 . Eq. 5.38 provides precise information about the required controller parameter variation for a COFB system where the machine optics changes over time but the ORM is not updated accordingly i.e. $\rho(\mathbf{M})$ increases over time from 0 to 1 . In this case, the controller parameters are needed to be scaled with $(1-\rho(\mathbf{M}))$ relative to the controller parameters corresponding 


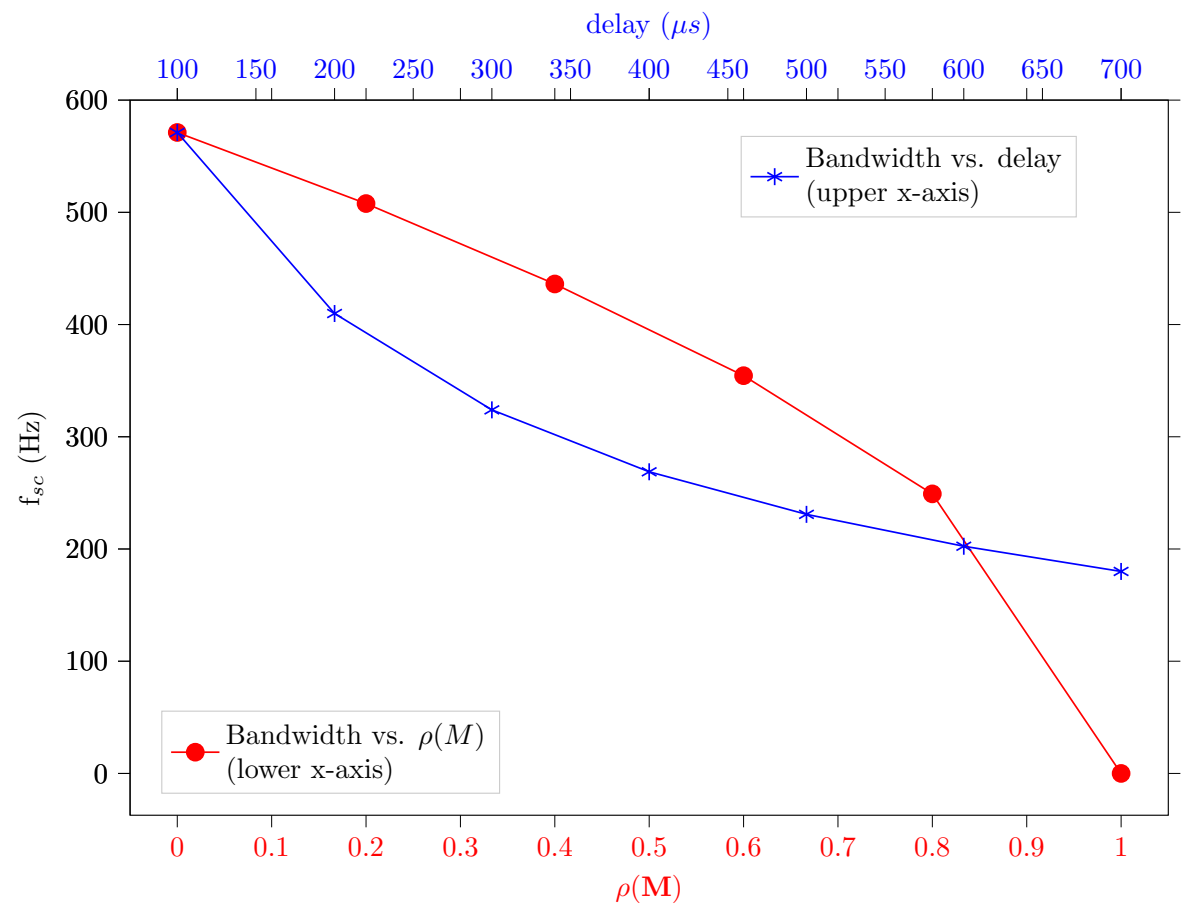

Figure 5.17 Reduction in the cut-off frequency as a function of $\rho(\mathbf{M})$ (lower $x$-axis) and delay (upper $x$-axis).

to $\rho(\mathbf{M})=0$.

In order to demonstrate the need of such a scaling, three first-order systems are considered with bandwidth of $850 \mathrm{~Hz}$ and delays $\tau_{d}=200,400$ and $600 \mu \mathrm{s}$. The PI controller corresponding to $\rho(\mathbf{M})=0$ for each system is optimized using IMC-tuning (discussed in sec. 2.8.4), let us call it $k_{0}(z)$. According to Eq. 5.38, the optimal controller gains $k_{\rho}(z)$ should be less than $k_{0}(z)$ by a factor $(1-\rho(\mathbf{M}))$. This directly implies that keeping the controller gains fixed at $k_{0}(z)$ for $\rho(\mathbf{M})>0$, the effective gains $k_{e f f}(z)$ are more than the the required gains by a factor as,

$$
k_{e f f}(z)=\frac{k_{0}(z)}{(1-\rho(\mathbf{M}))}
$$

For the above mentioned three first-order systems, the controller gains were optimized and then scaled according to Eq. 5.41 in order to incorporate the effect of spatial model mismatch. $\rho(\mathbf{M})$ was varied between 0 and 1 and the effective controller $k_{e f f}(z)$ was used to calculate the complementary sensitivity function $T(z)=\frac{L(z)}{1+L(z)}$ (Eq. 5.39 .

Fig. 5.18 shows the dominant pole positions of $T(z)$ on the complex plane of Laplace domain (in order to plot the frequency in $\mathrm{Hz}$ using transformation of $z=e^{s T_{s}}$ ) for all three systems. The pole-position on the right hand side of the complex plane (positive decay constant) shows the instability of the system as discussed in sec. 2.8.1. The poles move from left (stable region) to the right (unstable region) of the complex plane with an increase of $\rho(\mathbf{M})$ for all three cases whereas the cross-over value of $\rho(\mathbf{M})$ depends upon the dynamics (delays in this case) of the system. Higher is the delay, smaller is the value of $\rho(\mathbf{M})$ that can make the closed-loop 


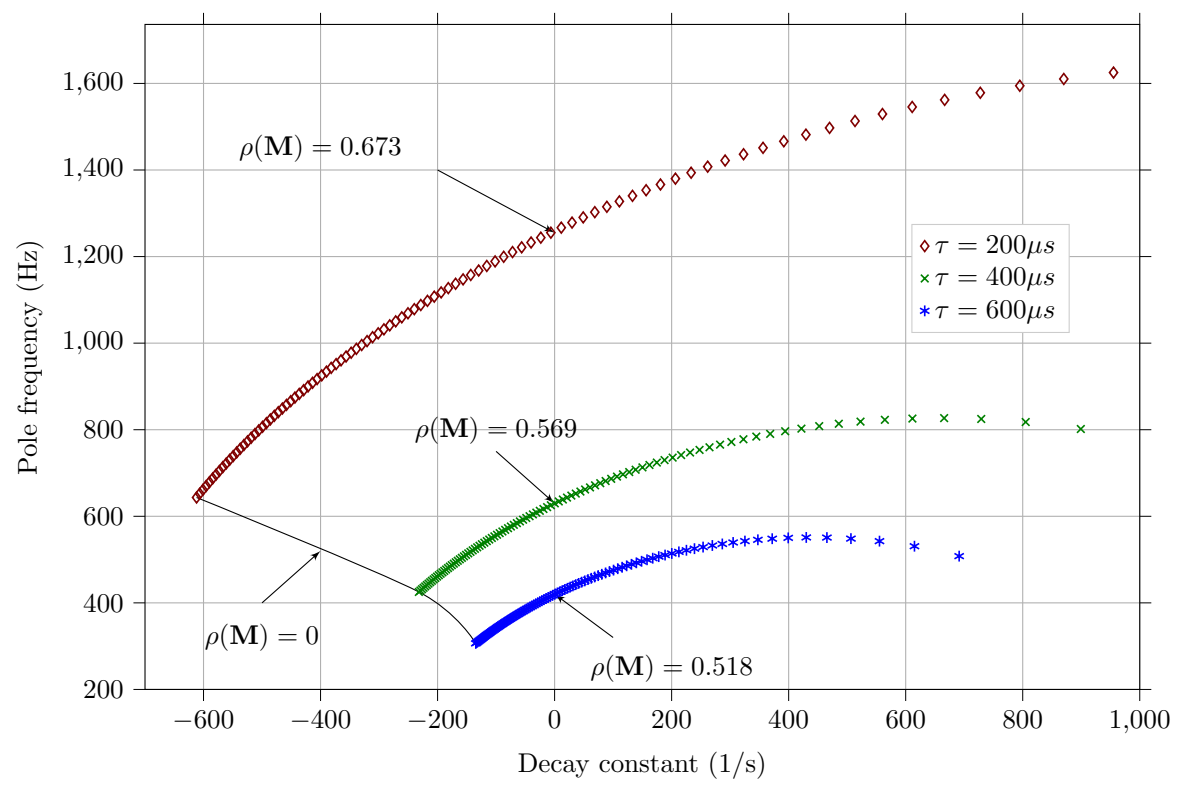

Figure 5.18 The closed-loop poles of complementary sensitivity function moving from left- to the right-hand side the the complex plane with the increase of spatial model mismatch characterized by $\rho(\mathbf{M})$. Three open-loop systems are considered having different pure delays.

unstable. These simulations confirm that spatial model mismatch beyond a certain threshold of $\rho(\mathbf{M})$ defined by the dynamics of the system, can result in the COFB system instability if the the controller parameters are not scaled according to Eq. 5.38. This conclusion has most relevance for SIS18 COFB system to run it in a fixed-ORM mode throughout the ramp, when the machine optics varies over time.

It is important to mention that the instability predicted for $\rho(\mathbf{M})<1$ in Fig. 5.18 is not in contradiction to the condition of inequality 5.12 which is defined for the slow regime where the dynamics of the system can be ignored and there is no need of scaling of the controller parameters. One can see that the threshold of $\rho(\mathbf{M})$ in Fig. 5.18 moves towards 1 as the delay of the system decreases relative to the sampling time $T_{s}=100 \mu \mathrm{s}$. On the other hand, if the controller parameters are scaled according to Eq. 5.38 as a function of model mismatch in the fast regime, the COFB instability due to the model mismatch will occur only for $\rho(\mathbf{M})>1$ (referred to Eq. 5.36), resulting in the identical condition for instability for both slow and fast regimes.

\subsection{Summary and contributions}

The contributions of the work presented in this chapter are listed as below, out of which many are also published in $[88]$.

- The effect of spatial model mismatch on the stability and the performance of the COFB 
systems is investigated for the slow and the fast regimes of orbit correction, separately, for the first time in a systematic manner.

- In the slow regime, the temporal dynamics of the system can be ignored in comparison to the slow rate of orbit correction and the closed-loop response depends only on the orbit response matrices of the controller and the system. For this regime, the spectral radius condition of stability is defined for the correction matrix $\mathbf{M}$ which yields a higher and practical stability margin in comparison to the previously used small gain theorem, against the spatial model mismatch.

- It is shown that the stability margins yielded by the two conditions can be related through the condition number of the nominal (controller) ORM, with exact equality for the circulant symmetry.

- A relation of the spectral radius $\rho(\mathbf{M})$ with the correction ratio is also established.

- The spectral radius is calculated for various sources of model mismatch in SIS18 in order to obtain hints for the optimal design of a COFB system. For on-ramp ORM variation at SIS18 due to triplet to doublet quadrupole transition, it is shown with the help of simulations in MAD-X that the defined spectral radius is a practical figure of merit for quantifying the spatial model mismatch.

- The simulations predicted that SIS18 COFB system will not be unstable even for the maximum on-ramp model mismatch for the slow regime of operation in both planes.

- The calculations of spectral radius are also performed for intensity-dependent tune shift and the beta-beating in SIS18. It is shown that the spectral radius is related to the magnitude of the tune shift and more sensitive in the direction towards the integer value of tune, and hence the instability threshold to spatial model mismatch depends on the working point.

- It is found that beta beating itself is not a crucial source of model mismatch unless accompanied by a tune shift towards an integer value.

- The conclusions drawn from the calculations and simulations for SIS18 were experimentally verified at COSY FZJ where the closed orbit is made unstable with artificially induced tune shift-based model mismatch. The observed instability threshold is in agreement with the instability threshold predicted by the spectral radius condition.

- These results from the slow regime are extended to the fast feedback regime also accounting for closed-loop dynamics. It is found that the spectral radius condition defines the stability condition of the fast COFB system for a given temporal system model.

- The controller settings must be scaled with $\rho(\mathbf{M})$ for a fixed-ORM mode of the COFB system for a variable optics machine, in order to ensure the closed-loop stability. 
- The achievable system bandwidth can be calculated from $\rho(\mathbf{M})$ and in order to achieve a required bandwidth, $\rho(\mathbf{M})$ can be controlled by the appropriate updating of the ORM in the controller of the COFB system.

- In nutshell, a single measurable parameter, i.e. spectral radius of the correction matrix $\mathbf{M}$ quantifying the model mismatch is enough for predicting the residual orbit, bandwidth reduction and the stability criteria in terms of controller parameters for the feedback system in consistency with the practical limits. 


\section{Chapter Six}

\section{Hardware for SIS18 COFB system}

This chapter introduces the hardware used for the implementation of closed orbit feedback controller for SIS18. Primary modules and functionalities implemented in the hardware are described. The tests performed on the hardware during commissioning are also mentioned.

\subsection{Basic hardware requirements}

In chapter 3, the general performance requirements of SIS18 COFB system are described and the developments made regarding orbit correction methods, uncertainty modeling in the ORM and the consequent stability and performance parameter i.e. $\rho(\mathbf{M})$ for the COFB systems were worked out in subsequent chapters, in line with general requirements. The conclusions drawn based upon theoretical investigations and simulations provide a basic understanding of the requirements for the hardware capable of implementing such a COFB system. The major demands can be described in a broad way as follows:

- Calculation rate: Based upon the frequency spectrum of closed orbit disturbances (see Fig. 3.6), initially a COFB system-bandwidth as high as possible up to $1 \mathrm{kHz}$ was foreseen. For this purpose, a controller hardware capable of corrector-strength calculation at a rate higher than $2 \mathrm{kHz}$ is needed so that the hardware does not put any limitation for the achievement of foreseen bandwidth.

- ORM update: The possibility of ORM update during an acceleration cycle, in the controller hardware is also a fundamental requirement for the SIS18 COFB system, particularly in the horizontal plane. Although the simulations have predicted that the ORM update is not required at every instant (milliseconds) of ramp and the maximum model mismatch characterized by the $\rho(\mathbf{M})$ is less than 1 . But in order to avoid bandwidth reduction due to the spatial model mismatch and the instability in the fast regime even for $\rho(\mathbf{M})<1$, an ORM update is recommended, during the ramp.

- Interface with power converters: The digital values of the corrector-strengths also need to be sent to the power converters of the corrector magnets with an appropriate interface to have the pure delay as minimum as possible.

One trivial requirement from COFB system hardware to be integrated with FAIR accelerators is as follows: 
- Synchronization with machine cycles: All accelerators of FAIR project are connected to a centrally dedicated timing system called General Machine Timing System (GMT), the details are given in Appendix A.1. GMT is responsible for the timely execution of the pre-planned actions of each component and provides the possibility of cascading the beam processes in time e.g. acceleration of beam in UNILAC (Universal Linear Accelerator), injection to and extraction from SIS18 etc. COFB system hardware also needs to have synchronization with GMT for BPM data acquisition and calculation of corrector strengths.

\subsection{Hardware for SIS18 COFB system}

Libera Hadron PlatfomB (called Libera Hadron onward) is the device used for the SIS18 COFB system and is developed for orbit correction at hadron synchrotrons by the Instrumentation Technologies (I-Tech) [89] Slovenia. It is delivered as an in-kind contribution to the FAIR project and was never tested and commissioned in a COFB system, before. In this way, one natural scope of the thesis is also to verify the appropriateness of Libera Hadron for COFB systems of SIS18 and SIS100 and to suggest further improvements. Libera Hadron is the advanced version of already existing Libera PlatformA which is used for the analog to digital data processing of the BPM plate signals. Libera Hadron is also capable of BPM data processing along with additional features of corrector-strength calculation and making them available to the power converters of the corrector magnets at a rate of $10 \mathrm{kHz}$. Dedicated modules are installed for each of the above mentioned actions, the details are given in Appendix A.2.

\subsubsection{Prime modules of Libera Hadron}

Libera Hadron has an FPGA board for the controller implementation in its GDX module, in order to achieve low latency in corrector-strength calculation and is FPGA-programmed also by the I-tech, Slovenia. Three important modules relevant for the corrector-strength calculation along with their functionalities are listed below:

- Four Hadron Beam Position Monitor (HBPM) modules each for the BPM data processing. HBPM modules replace the previously used Libera platformA for receiving the signal from upto four BPM plates (also called pick-ups), doing analog-to-digital signal processing and providing a $10 \mathrm{kHz}$ data stream for the closed orbit correction.

- Gigabit Data eXchange (GDX) module is the main module of Libera Hadron for the exchange of beam position data with other Libera Hadrons and for the implementation of the controller algorithm and storing the inverse ORM for corrector-strength calculation.

- Serial (SER) interface module for supplying current values to the corrector power converters. SER module of each Libera Hadron has maximum 8 serial ports so that maximum 8 power converters can be connected to one Libera Hadron. 


\subsubsection{Functionalities implemented in GDX module}

Here we discuss the salient features of the algorithm implemented in the GDX module of Libera Hadron for the corrector-strength calculation, in the context of SIS18 COFB system. The update rate of $10 \mathrm{kHz}$ is already higher than that required for a bandwidth of $1 \mathrm{kHz}$. The data flow withing the Libera Hadron from BPM readouts to the corrector-strengths is shown schematically in Fig. 6.1 while various data symbols are explained in Table. 6.1. Each of the relevant design feature of the GDX module is discussed below. The details can be found in the user manual of the Libera Hadron [90] as well as in [91].

\section{Global orbit data}

Although each Libera Hadron can receive data from a maximum of 4 BPMs (due to 4 HBPM modules per Libera), the implementation of "Libera Grouping Protocol" implemented in the GDX module provides the possibility of creating a global orbit vector from all BPMs. As a result, a combination of several Libera Hadrons can form a global COFB system. In the grouping network, global orbit data packets are passed from GDX module of one Libera Hadron to the next where each GDX inserts the sum, position and status data of its connected HBPM modules. The latency of the Libera Grouping is in the range of $0.1 \mu$ s per BPM and thus distribution of global orbit data can take place within $10 \%$ for SIS100 and $2 \%$ for SIS18, of the $100 \mu$ s time slice for orbit correction. Synchronism between all the HBPM modules is required and achieved by starting the data chains at a specific time on all Libera Hadrons using trigger from the timing system. The timing modules of Libera Hadron are discussed in Appendix A.2. Consequently, each Libera Hadron calculates the strengths for all correctors in parallel. It is important to mention that the calculation is performed only for one plane per Libera Hadron i.e. either horizontal or vertical.

\section{Double buffering}

An important feature of the Libera Hadron is the possibility to store two ORMs, reference orbits and beam rigidity vectors in two data buffers in the FPGA of the GDX module (Fig. 6.1). During the orbit correction, one buffer is active and the other is available for parameter loading. In this way, ORMs can be updated occasionally in a pipe-lined manner in accordance with the optics change during the ramp in SIS18 (for details see sec. 3.2). For initial tests, there were two limitations regarding ORM update:

- The buffers in the FPGA of the GDX module are programmed only for the real valued SVD matrices of the ORM i.e. $\mathbf{S}^{+} \mathbf{U}^{\mathbf{T}}$ and $\mathbf{V}$ and there is no possibility of uploading the complex matrices of the DFT-based decomposition discussed in chapter 4 . This means that ORM update only in terms of the complex Fourier coefficients instead of all three SVD matrices could not be tested experimentally.

- There was no user interface for the upload of the ORMs and switching the active buffer during the fast ramps (200 ms - $1 \mathrm{~s}$ ), at the time of measurements presented in this thesis. That's why initial tests are planned for using a single ORM during the ramp. 


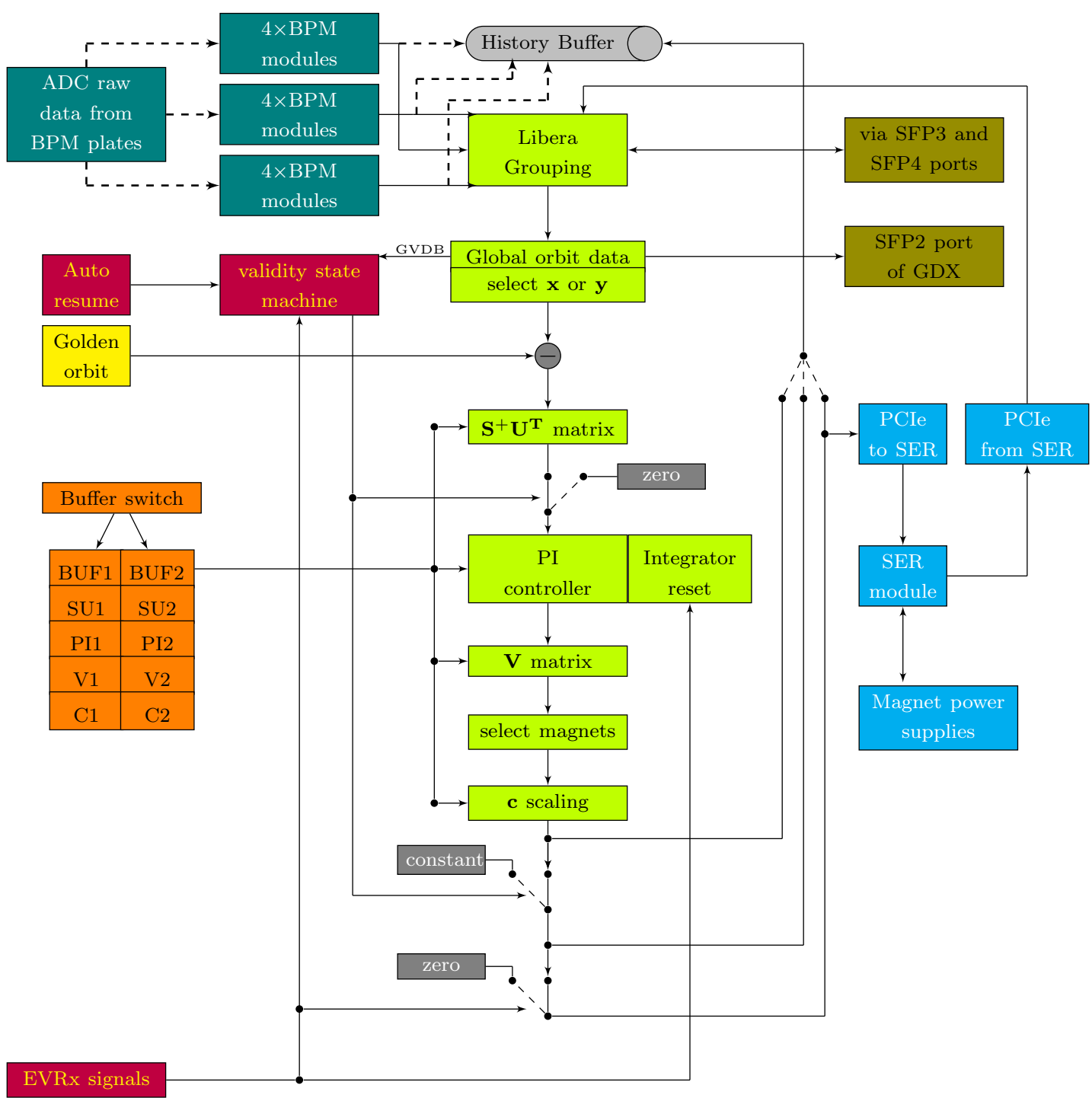

Figure 6.1 SIS18 COFB design overview. For the meanings of symbols see Table 6.1

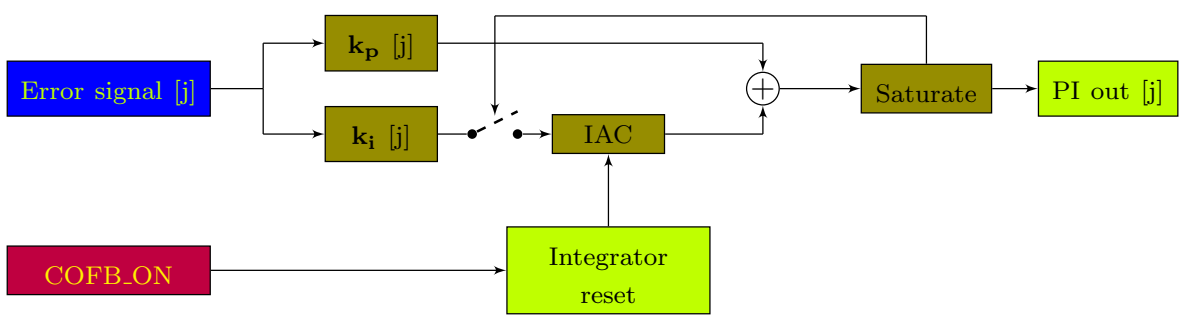

Figure 6.2 Schematic of the PI controller for $j^{\text {th }}$ SVD mode. IAC stands for Integrator accumulator.

\section{Corrector-strength calculation}

The corrector-strength calculation is performed in the GDX module of Libera Hadron where the SVD-decomposed matrices can be stored by the user in the form of $\mathbf{S}^{+} \mathbf{U}^{\mathbf{T}}$ and $\mathbf{V}$. The 
Table 6.1 The symbols of the signals and matrices used in the COFB algorithm.

\begin{tabular}{ll}
\hline \hline Symbol & Description \\
\hline \hline $\mathbf{x}$ or $\mathbf{y}$ & Measured orbit array \\
\hline $\mathbf{r}$ & reference orbit array \\
\hline $\mathbf{S}^{+}$ & Pseudo-inverse of SVD singular matrix \\
\hline $\mathbf{U}$ & SVD left orthogonal matrix \\
\hline $\mathbf{k}_{\mathbf{p}}$ & Proportional gains column of PI controller \\
\hline $\mathbf{k}_{\mathbf{i}}$ & Integral gains column of PI controller \\
\hline $\mathbf{V}$ & SVD right orthogonal matrix \\
\hline $\mathbf{c}$ & Scaling for radian to current \\
\hline $\mathrm{M}$ & Number of BPMs \\
\hline $\mathrm{N}$ & Number of corrector magnets \\
\hline $\mathbf{j}$ & Index of SVD mode \\
\hline
\end{tabular}

dimension of each matrix is $128 \times 128$ which is larger than required even for SIS100 where the number of BPMs and correctors are 84 each, while for SIS18 the ORM dimension is $12 \times 12$. Instead of any alteration in the FPGA programming for the dimension of the ORM, it is decided to store the SIS18 ORM in the full dimension by setting the remaining elements equal to zero, the calculations are verified in Python, beforehand. Similarly, the dimension of global orbit vector is also kept equal to 128 while only first 12 elements contained beam position from 12 BPMs of SIS18.

The sequence of calculation of corrector-strengths is shown in Fig. 6.1 where the output of $\mathbf{S}^{+} \mathbf{U}^{\mathbf{T}}$ matrix is fed to a Proportional-Integral (PI) controller featuring the anti-windup algorithm, the schematic is shown in Fig. 6.2. Whenever the PI controller output saturates (the limit defined by the user), the Integrator accumulator (IAC) is held constant at the last stored values. The integrator is reset at each restart of the COFB system. The controller parameters are stored in one-dimensional arrays $\mathbf{k}_{\mathbf{P}}$ and $\mathbf{k}_{\mathbf{i}}$ of the size equal to the total number of possible SVD modes of the ORM i.e. 128. Such an implementation provides the liberty of using different controller gains for each of the SVD mode, a unique feature of this implementation.

The output of PI controller is multiplied with $\mathbf{V}$ matrix and the calculated corrector-strengths (radian) are scaled with the beam rigidity given as $\mathbf{c}$ scaling. The corrector-strengths are calculated for all correctors on each Libera Hadron but only those are sent to the SER module for which its 8 output ports are configured, beforehand.

\subsection{Testing of Libera Hadron during commissioning}

As mentioned before, Libera Hadron being a new development, was not used at any other facility in the functional COFB system, at the time of commissioning of SIS18 COFB system. Therefore, a number of tests were needed to be performed before its deployment in the COFB 

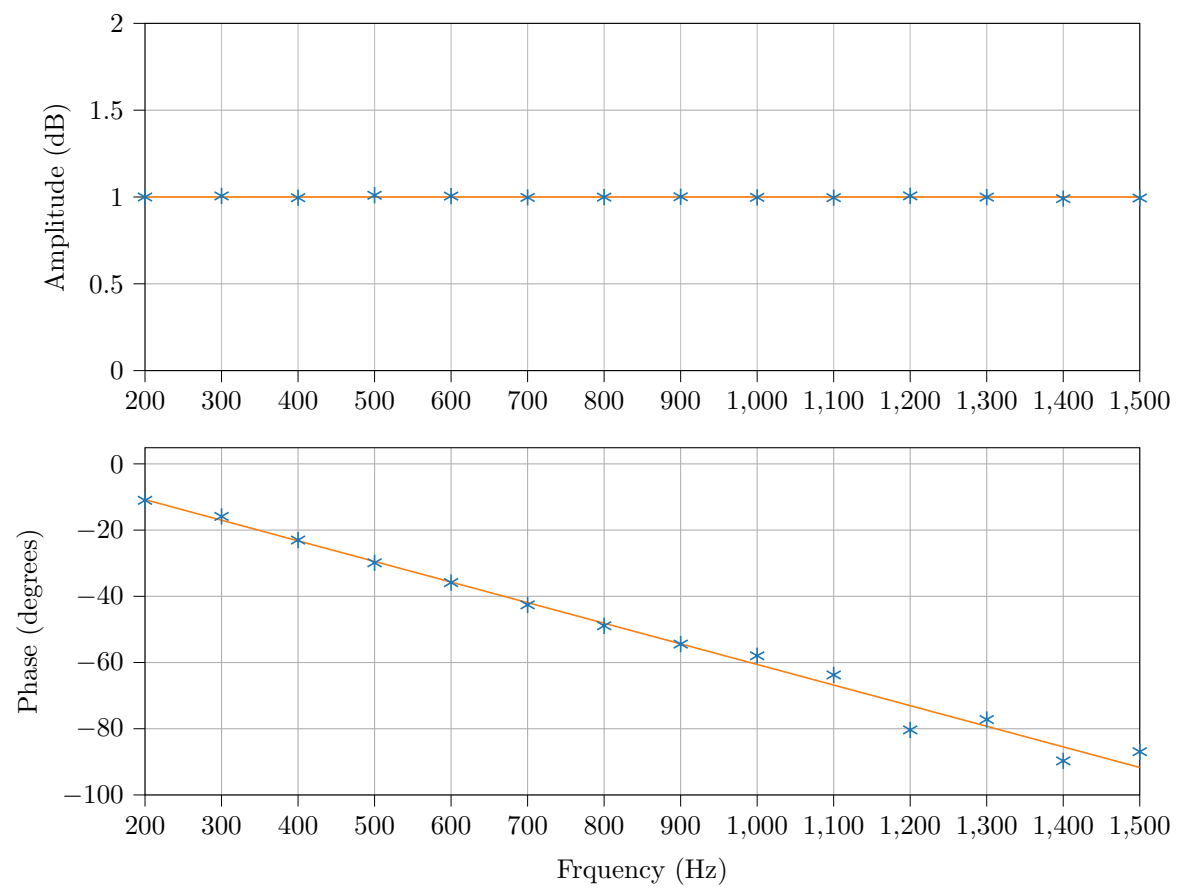

Figure 6.3 Frequency response of Libera Hadron and DAC card. The points show the measurement while the lines show the linear fit.

system. The tests included the measurement of the pure delay from BPM signal input to the corrector-strength output and correctness of the corrector-strength calculation. Some additional features were also demanded and got implemented during the tests.

\subsubsection{Delay measurement}

In order to measure the time delay contribution of Libera Hadron in the feedback loop of SIS18 COFB system, a signal generator is used to mimic the BPM data, artificially, in the absence of beam. Amplitude modulation of a range of frequencies from 200 to $1500 \mathrm{~Hz}$ is done over a base frequency of $300 \mathrm{kHz}$ (typical bunch revolution frequency in SIS18 at injection). Consequently, artificial closed orbit oscillation of the modulating frequencies is fed to the calculation algorithm in the Libera Hadron. The range of modulating frequencies is chosen to cover the expected bandwidth of the COFB system. The digital output from one of the SER modules are displayed on oscilloscope with the help of digital-to-analog converter (DAC) card whose time delay is separately measured to be $50 \pm 10 \mu \mathrm{s}$. The output of DAC is compared with the modulating input signal on an oscilloscope, for the pure delay measurement. The transfer function representation of the pure delay $\tau$ can be written in frequency domain for $s=j \omega$ as,

$$
e^{-\tau s}=e^{-\tau j \omega}=\cos (\omega \tau)-\sin (\omega \tau)
$$


The phase difference between input and output sinusoidal signals for the transfer function of Eq. 6.1 is,

$$
\angle e^{-\tau j \omega}=\Delta \phi(\omega)=-\omega \tau
$$

which shows that $\tau$ is equal to the slope of the straight line between phase difference (radian) and the input angular frequency (radians per seconds). The measured amplitude ratio of input and output signals as well as the phase difference is plotted in Fig. 6.3. One can see that there is no amplitude attenuation of the signal in the Libera Hadron but a pure delay is obvious that is calculated from the measured phase difference to be equal to $110 \pm 10 \mu \mathrm{s}$, excluding the delay caused by DAC. It is important to mention that the measured delay of Libera Hadron is mainly due to the averaging done for $100 \mu$ s for the closed orbit calculation to provide data at $10 \mathrm{kHz}$ and the delay of corrector-strengths calculation is much smaller than that.

\subsubsection{Fixed-point arithmetic test}

An important parameter of performance for Libera Hadron is the digital signal processing (DSP) during the corrector-strength calculation in the FPGA of GDX module. The algorithm is implemented using fixed-point arithmetic, which provides a finite number of bits (register width) to perform arithmetic operations at each step of the correction-settings calculation. It is contrary to the floating-point arithmetic where the numerical representations of numbers in binary format can ideally have infinite bits (e.g. working with pencil and paper or in higher-level floating-points environments such as MatLab etc.). In the fixed-point $N$-bit binary representation, a signed integer $i$ can have a value only in the range $-2^{N-1} \leq i \leq 2^{N-1}$. Some relevant attributes of fixed-point arithmetic are given as [92],

- Precision is the maximum number of non-zero bits that can be represented for a given register width.

- Resolution is the smallest non-zero magnitude that can be represented in a given register width.

- Range is the difference between the most negative and most positive numbers represented in a given register width.

- Accuracy is the maximum difference between the real value of a number and its binary representation.

- Dynamic range is the ratio of the maximum and minimum absolute values that can be represented in a given size of bits. For a $N$-bit binary representation, the dynamic range is 0 to $2^{N-1}$.

- Sum or Difference of two $N$-bit signed numbers requires $N+1$ bits.

- Multiplication of two $N$-bit and $M$-bit signed number requires $N+M$ bits. 
Table 6.2 Available register width and bit cutting at each node of the correctorsettings calculation. The center value in the third column is retained while left and right number of bits are cut $[90]$. For meaning of the symbols see Table. 6.1.

\begin{tabular}{lll}
\hline \hline Calculation node & $\begin{array}{l}\text { Register } \\
\text { width }\end{array}$ & Bit cutting \\
\hline Global orbit, $\mathbf{p}$ & 32 & $6-21-5$ \\
\hline $\mathbf{d P}=\mathbf{r}-\mathbf{p}$ & 22 & $0-22-0$ \\
\hline $\mathbf{S}^{+} \mathbf{U}^{\mathbf{T}}$ matrix & 18 & $0-18-0$ \\
\hline$\left[\mathbf{S}^{+} \mathbf{U}^{\mathbf{T}}\right] \mathbf{d p}$ & 48 & $10-22-16$ \\
\hline $\mathbf{k}_{\mathbf{p}}$ and $\mathbf{k}_{\mathbf{i}}$ arrays & 18 & $0-18-0$ \\
\hline$\left[\mathbf{S}^{+} \mathbf{U}^{\mathbf{T}} \mathbf{d p}\right] \mathbf{k}_{\mathbf{p}}$ & 40 & $0-40-0$ \\
\hline$\left[\mathbf{S}^{+} \mathbf{U}^{\mathbf{T}} \mathbf{d p}\right] \mathbf{k}_{\mathbf{i}}$ & 40 & $0-40-0$ \\
\hline Integration & 41 & $1-40-0$ \\
\hline Sum after PI & 41 & $3-22-16$ \\
\hline $\mathbf{V}$ matrix & 18 & $0-18-0$ \\
\hline$[\mathbf{V}]$ PI output & 48 & $8-20-20$ \\
\hline $\mathbf{c}$ array & 18 & $0-18-0$ \\
\hline$[[\mathbf{V}]$ PI output $] \mathbf{c}$ & 38 & $8-20-10$ \\
\hline
\end{tabular}

Since, the multiplication has to be performed at different stages of the algorithm for the corrector-settings calculations, a finite register width at each calculation node will require a bit cutting of the resulting signal, if the available register width is less than the sum of number of bits of two multiplying signals. This requires a careful allocation of register width at each calculation node in order to avoid the loss of significant information and a wrong corrector-strength calculation. Moreover, in order to use the whole dynamic range available at each calculation node, the elements of the SVD matrices and the arrays of controller parameters need to be normalized with the available dynamic range, before uploading. In order to verify the correctness of calculation and the appropriateness of register width at each node of the calculation, the algorithm implemented in the FPGA of GDX module was simulated and the corrector-settings were calculated for a static closed orbit distortion (upto $30 \mathrm{~mm}$, shown in Fig. 6.4 top) produced artificially with the help of signal generator, in the absence of beam. First of all, the comparison of the simulated output with the corrector-settings calculated in the Libera Hadron helped to bench mark the simulation against the real implementation.

As a second step, the comparison of the simulated output at each node with the values calculated directly, without normalizing and bit cutting of the closed orbit and the ORM, helped to testify the appropriateness of bit width at each calculation node. Changes in the register width at some of the nodes was recommended, as a result of above comparison, and was re-implemented by I-tech, accordingly. For example, originally the $\mathbf{S}^{+} \mathbf{U}^{\mathbf{T}}$ matrix was normalized to the dynamic range of 12-bit while a 18-bit was demanded for better resolution. Similarly, register width is increased from 22 to 40 for the multiplication of 

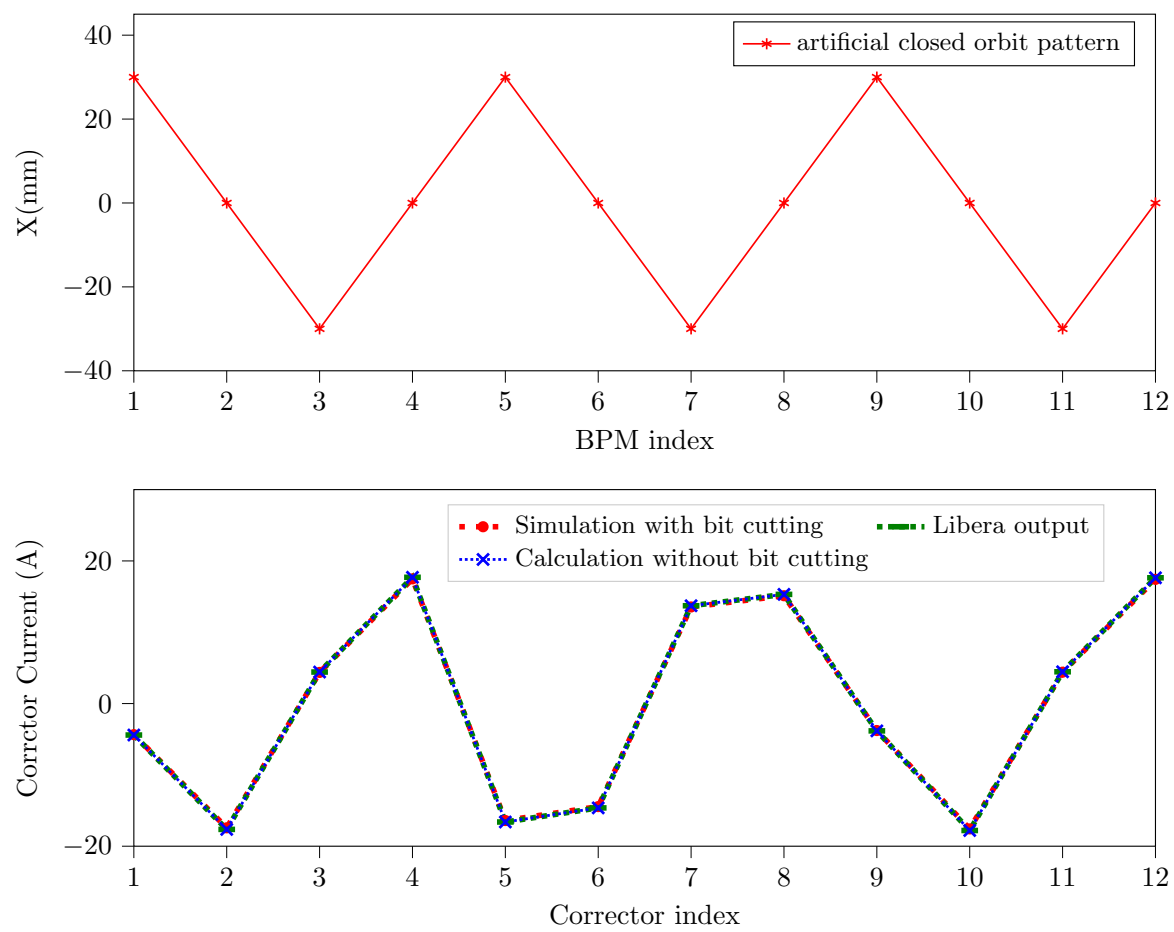

Figure 6.4 Comparison of corrector-settings calculation between Libera Hadron, simulation with bit cutting and calculation without bit cutting.

signal with controller parameter $\mathbf{k}_{\mathbf{i}}$ since the saturation was observed at this node, later during beam-based tests. The finally implemented register width at each calculation node is shown in Table. 6.2. The comparison between corrector-strengths calculated by all above mentioned three methods is shown in Fig. 6.4 for the artificially generator closed orbit pattern, demonstrating the faultlessness of corrector-strength calculation in Libera Hadron, before commissioning of the COFB system.

\subsubsection{Synthetic data generator for system identification}

In order to measure the orbit response matrix and the temporal response of the corrector magnets as well as for the full system identification, a synthetic signal generator in the SER module was recommended and was installed by the I-tech during the tests of the Libera Hadron. The synthetic generator replaces the real magnet correction data coming from the COFB algorithm with the user defined synthetic data.

\subsection{Summary}

The main hardware used for the SIS18 COFB system is Libera Hadron developed by I-tech Slovenia and is an in-kind contribution to the FAIR project. It is a single device containing the dedicated modules for obtaining real time machine events, acquisition and processing 
of beam position data and calculating the corrector-strengths at $10 \mathrm{kHz}$ with the help of a Proportional-Integral (PI) controller and supplying to the power converters of corrector magnets. The appropriateness of Libera Hadron for SIS18 COFB system was verified in line with general performance requirements and theoretical investigations and simulations presented in previous chapters. 


\section{Chapter Seven}

\section{SIS18 COFB system in horizontal plane: orbit correction and characterization}

This chapter presents the commissioning of the SIS18 COFB system and the on-ramp orbit correction only in the horizontal plane due to the lack of adaptive control units for the power converters of vertical corrector magnets at the time of thesis. Measurement of the frequency response of component hardware as well as full-loop system identification is described. Orbit response matrices are measured over the ramps of two different machine settings for Argon and Uranium beams respectively, and the spatial model (i.e. ORM) mismatch is calculated with respect to the injection ORM. The orbit correction is demonstrated with a fixed injection ORM and for the fixed controller gains for each ramp. The effect of spatial model mismatch on the stability and performance of the feedback system is investigated in the light of conclusions drawn in Chapter 5 .

\subsection{Components of the COFB system}

The hardware components of the COFB system realized in the horizontal plane of SIS18 include:

- 12 Shoe-box type beam position monitors (BPMs) (for details see sec. 2.8.3) one in each section of the SIS18 synchrotron. BPMs had transformers installed for the impedance coupling between the BPM plate and the $50 \Omega$ side of the electronics, at the time of measurements presented in this thesis. They were installed on SIS18 BPMs to test them for their future use in SIS100.

- Three Libera Hadrons, one for 4 BPMs in which data is digitized at $250 \mathrm{MSa} / \mathrm{s}$ and then processed at $10 \mathrm{kHz}$ for the COFB system. The corrector-strengths are also calculated at the same rate of $10 \mathrm{kHz}$. The detail of the hardware is already described in Chapter 6 and Appendix A.2.

- $30 \mathrm{~m}$ long Cat6 (Ethernet) cables, one for each corrector-strength output to be transmitted from Libera Hadron to the adaptive control unit of the corrector magnet.

- 12 adaptive control units (ACUs) for converting digital values of the corrector-strengths from Libera Hadron into analogue values of the corrector magnet current (ampere). 
Table 7.1 Comparison of SIS18 and SIS12 modes of main dipole configuration $\mid 93$.

\begin{tabular}{lll}
\hline \hline Parameter & SIS18 mode & SIS12 mode \\
\hline Peak magnetic field & $1.8 \mathrm{~T}$ & $1.2 \mathrm{~T}$ \\
\hline Magnet ramping rate & $4.2 \mathrm{~T} / \mathrm{s}$ & $10 \mathrm{~T} / \mathrm{s}$ \\
\hline power converter & 4 sets each of 6 & 2 sets each of 12 \\
configuration & power converters & power converters \\
& connected in series & connected in parallel \\
\hline
\end{tabular}

- 12 power converters for the horizontal corrector magnets.

- 12 corrector magnets which are extra winding on the main dipoles of SIS18 for the horizontal plane.

\subsection{Open-loop system identification}

System identification is the process of mathematical modeling of system's dynamic response using measurements of the system's input and output signal. The input signal can be sinusoidal, step or pulse function of time while the outcome of system identification is the frequency response of the system (sec. 2.8.1). The design, optimization and performance of the controller strongly depend upon the frequency response of the system to be controlled. The mathematical model of the system's response helps to predict the controller parameters in advance (mostly through simulations) in order to ensure the closed loop stability within the limits of achievable performance. For the COFB system, the corrector magnets and their power converters are the major contributors in defining the overall frequency response of the system and the pure delay of the loop. As the first step, the frequency responses of all 12 horizontal corrector magnets along with their power converters are measured one by one. Since, the horizontal corrector magnets are extra winding on the main dipoles, the measurements are performed for the two modes of the main dipole power converters i.e. slow and fast modes called SIS18 and SIS12 modes (comparison given in table 7.1), respectively. The purpose of the measurement is to investigate the effect of mutual induction between winding of corrector magnets and main dipole magnets. The measurements are carried out using the sinusoidal excitation of discrete frequencies as well as pulse inputs of various heights. As a second step, the beam-based measurement of the combined frequency response of all hardware in the loop are also made using the sinusoidal excitation of discrete frequencies for each corrector channel. The details of the measurement are given in subsections below.

\subsubsection{Sub-system identification: Corrector magnets, power convert- ers and adaptive control units}

As a first step, the frequency response of the corrector magnets and associated devices including power converters and adaptive control units are measured. The electronic architecture of the hardware components of these devices are out of the scope of the thesis and details can be 
found in 94 . Here, the features of these devices relevant to COFB system are listed while a few more details are mentioned in Appendix B.1.

- The ACU is a modular digital control system for corrector magnet power converters. It has a Multifunction Module that provides the user interface for manual operation, service and diagnosis by computer or oscilloscope. It also contains the communication ports to the external accelerator control system via the basic I/O-unit (Interface Card). Two independent PI control loops and the control topology of the power converter are also implemented in the Multifunction module.

- Universal Serial Interface (USI) supports communication between Multifunction module with SER module of the Libera Hadron at a baud rate upto $20 \mathrm{MBit} / \mathrm{s}$ with the help of Ethernet cable and RJ45 connector.

- Power converters have two special features: the rate limiter against the high slew-rate and the anti-oscillation (causing interlock) as the protection measures for the power converter hardware (diodes etc.).

\section{Frequency response measurement}

In order to measure the transfer functions of all 12 corrector magnets, their power converters and ACUs, the sinusoidal output of the synthetic generator implemented in the SER module of the Libera Hadron is used one by one for each corrector channel. The frequency of the sinusoidal signal of fixed amplitude $0.13 \mathrm{~V}(0.78 \mathrm{~A})$ is swept over a frequency range of $10-5000$ $\mathrm{Hz}$ with appropriate steps and the current in the magnet coil was monitored with the help of the DC current transformer (DCCT). The input signal and the output of the DCCT were also available on two Lemo jacks of the Multifunciton Module (reserved for diagnostic in Fig. B.1 of Appendix B of the ACUs that could be recorded on an oscilloscope. An external trigger was used for the measurement of the delay between input and output on oscilloscope. Figure. 7.1 shows the measured Bode plots (amplitude ratio and phase difference of output and input signals) for all 12 corrector magnets along with their individual power converters and ACUs, for the sinusoidal excitation of amplitude $0.78 \mathrm{~A}$. The dots in fig 7.1 represent the frequencies of measurement while the line interpolation is only for visual purpose. One can see that two out of 12 corrector channels have significantly different frequency response which is related to the fact that these two channels did not have ACUs rather had analog control loops along with DAC cards building a bridge between digital values sent from Libera and the analog set value fed to the analog control loop. The exact circuit design for these units is out of the scope of this thesis but the difference of their measured frequency response is of significance for the performance of the COFB. The measurement further shows that the magnitude response of majority of the correctors amplifies in the vicinity of $1.2 \mathrm{kHz}$ while attenuates below $-3 \mathrm{~dB}$ even after $2 \mathrm{kHz}$.

In order to extract the mathematical models of the transfer functions, the Bode plots of Fig 7.1 were fitted with the help of MATLAB function "tfest" used for the estimation of the transfer function from the measured data with following arguments,

$$
\text { sys }=\operatorname{tfest}(\text { data }, n p, n z)
$$



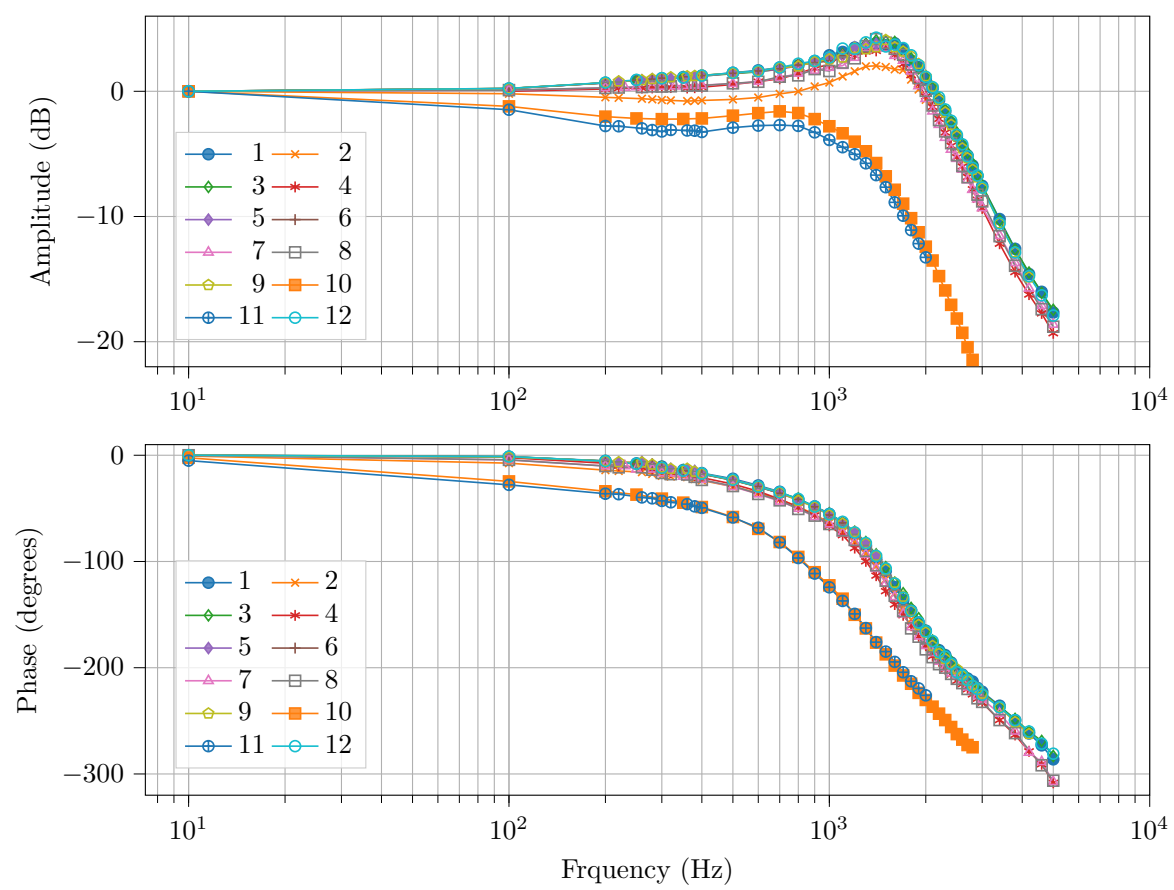

Figure 7.1 Measured Bode plots for all 12 horizontal corrector magnets along with their power converters and ACUs, in SIS18 mode.

where sys is the estimated transfer function and $n p$ and $n z$ are the expected number of poles and zeros, respectively. The data has to be supplied in complex form using the MATLab command "frd" as,

$$
d a t a=\operatorname{frd}\left(A_{r} e^{j \phi}, \omega\right)
$$

where $A_{r}$ is the amplitude ratio, $\phi$ is the phase difference and $\omega$ is the frequency in radians, from Bode plot.

The fitted Bode plots are shown in Fig. 7.2 for correctors marked as 4 and 11 in SIS18, as the representatives of two groups of Fig. 7.1, one comprising of correctors 1 to 9 and 12 and the other group containing correctors 10 and 11, respectively. The following transfer functions were estimated in continuous Laplace domain ' $s$ ' (in units of radians) and are written in zero-pole-gain form as,

$$
\begin{aligned}
& \text { subsys } 4=e^{-0.0001 s} \frac{2149.2\left(s+4.585 \times 10^{4}\right)(s+697.2)}{(s+737.7)\left(s^{2}+7245 s+9.723 \times 10^{7}\right)} \\
& \text { subsys } 11=e^{-0.00013 s} \frac{633\left(s+3.47210^{5}\right)(s+1069)}{(s+642.9)\left(s^{2}+7408 s+5.15 \times 10^{7}\right)}
\end{aligned}
$$

where subsys means the transfer function of the subsystem (corrector magnet, its power converter and ACU). It is important to mention that the symbol ' $\mathrm{s}$ ' in the transfer function is always Laplace variable and should not be confused with the symbol for second (time). The extracted parameters (poles and zeros) from Eqs. 7.3 and 7.4 are given in table 7.2. 

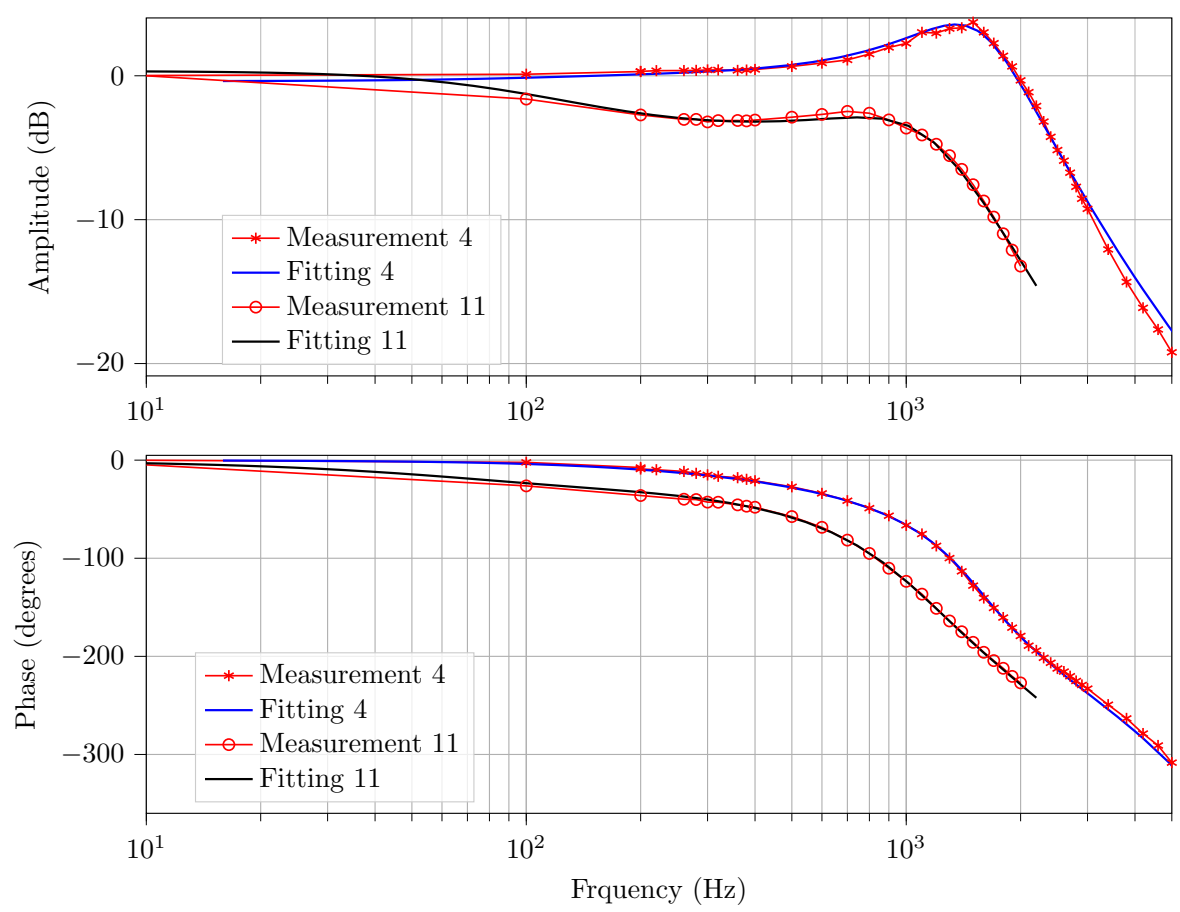

Figure 7.2 Measured and fitted (in MATLAB) Bode plots for corrector magnets 4 and 11 with their power converters.

For both groups of corrector channels, the real parts of all the poles lie on the left-hand side of the pole-zero complex plane (negative real parts) meaning that the system is open-loop stable (sec. 2.8.1). But the gain and phase margins for corrector 4 and hence for other nine similar correctors is quite small in comparison to the corrector 11, meaning less robustness against transfer function uncertainty. Corrector 11 has the exact bandwidth (defined by the $3 \mathrm{~dB}$ attenuation to input signal) of $207 \mathrm{~Hz}$ meaning that correctors 10 and 11 will behave "different" above this frequency in the orbit correction process and also destroy the assumption made for Eq. 2.72 that all corrector magnets have same temporal responses.

The Bode plots of Fig. 7.1 are measured for a very small amplitude (0.78 A) of sinusoidal excitation because at higher amplitudes and high frequencies, the power converters get switched OFF due to the anti-oscillation interlocks. Due to this reason, the measurement of Fig. 7.1 is not the representative of system's behavior against higher slew-rate for the corrector-strengths variation. In order to quantify slew-rate limited response, pulse inputs of variable amplitudes $(0.7 \mathrm{~A}, 6 \mathrm{~A}, 12 \mathrm{~A}, 18 \mathrm{~A}$ and $36 \mathrm{~A}$ reached within $10 \mu \mathrm{s})$ are applied and the measured system response for corrector 4 is plotted in Fig. 7.3 for both SIS18 (green) and SIS12 (blue) modes of the main dipole configuration. A similar measurement for corrector 11 is plotted in Fig. 7.4. The red curves show the input pulses in both figures. A ripple of fixed amplitude $(0.75 \mathrm{~A})$ and frequencies of $600 \mathrm{~Hz}$ and its higher harmonics are recorded in case of SIS12 mode (green in Figs. 7.3 and 7.4 of the main dipole configuration, for both corrector channels. Except this, there is no major difference for the rise time of the output response, for the two main-dipole configurations. On the other hand, the comparison of pulse 
Table 7.2 Parameters extracted from the system identification for the corrector magnets 4 and 11 along with their power converters. $p$ and $z$ represent the poles and zeros, respectively.

\begin{tabular}{llll}
\hline \hline Parameter & Unit & subsys4 & subsys11 \\
\hline \hline$p_{1}$ & $\mathrm{~Hz}$ & $-111+0 j$ & $-170+0 j$ \\
\hline$p_{2}$ & $\mathrm{~Hz}$ & $-576.5-1460 j$ & $-589.5-978.3 j$ \\
\hline$p_{3}$ & $\mathrm{~Hz}$ & $-576.5+1460 j$ & $-589.5+978.3 j$ \\
\hline$z_{1}$ & $\mathrm{~Hz}$ & $-7297+0 j$ & $-55250+0 j$ \\
\hline$z_{2}$ & $\mathrm{~Hz}$ & $-111+0 j$ & $-70+0 j$ \\
\hline delay & $\mu \mathrm{s}$ & 100 & 130 \\
\hline bandwidth & $\mathrm{Hz}$ & 2295 & 207 \\
\hline gain margin & $\mathrm{dB}$ & 0.578 at $2 \mathrm{kHz}$ & 7.12 at $1.4 \mathrm{kHz}$ \\
\hline phase margin & degrees & 5.24 at $1.9 \mathrm{kHz}$ & 169 at $37 \mathrm{~Hz}$ \\
\hline
\end{tabular}

response for step heights of different amplitudes shows a strong influence of the slew-rate of the input signal on the response of the system as can be seen by the different rise time of the output signal for different step heights. Such an influence is visible on both rising and falling edges of the pulse. The maximum allowed slew-rate measured is $20 \mathrm{kA} / \mathrm{s}=2 \mathrm{~A} / 100$ $\mu \mathrm{s}$. In this case, it would not be appropriate to represent the system's response with a single transfer function for any rate of change of corrector-strengths while on the other hand, it is not possible to take into account the rate of change of input signal for the transfer function which is by definition independent of input signal.

In order to further understand the dependence of the system's response on the slew-rate of the input signal, the Bode plots are extracted from the pulse responses plotted in Fig. 7.3 and 7.4 denoted by $u(t)$ from now onward, by calculating the impulse responses $h(t)$ by definition $(47])$ as,

$$
h(t)=\frac{d u(t)}{d t}
$$

As defined in sec. 2.8.1, that the transfer function of a system is the Laplace transform of its impulse response under assumption of zero initial conditions. Thereby, the frequency responses are calculated by the DFT of the impulse responses $h(t)$ calculated using Eq. 7.5 for the rising edge response of each input height (i.e. step response) and are plotted in Fig. 7.5. The frequency response measured by sinusoidal excitation for the amplitude of $0.78 \mathrm{~A}$ is also plotted for comparison which completely overlaps with the Bode plot calculated from the step response of same amplitude. The Bode plots extracted from step responses confirm a strong dependence of the system's frequency response on the rate of change of corrector-strengths (i.e. slew-rate). This means that with the existing power converters, system's effective transfer function can be variable over the ramp depending upon the slew-rate of controller output. This is in addition to the effect of spatial model mismatch discussed in chapter 5 .

The Bode plots of Fig. 7.5 are also fitted in MATLAB for the transfer function estimation (fitting not shown) and the dominant poles and zeros (for their meaning, see sec. 2.8.1) are 


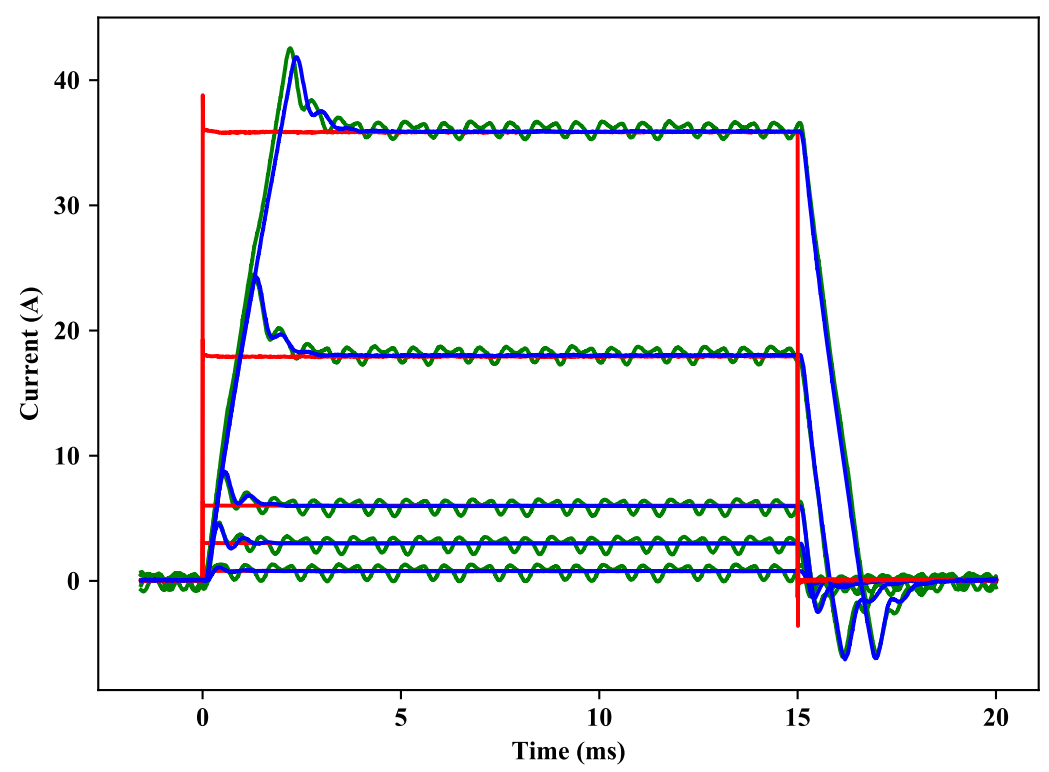

Figure 7.3 Pulse response of corrector magnet 4 along with its power converter and ACU for SIS18 (green) and SIS12 modes (blue) of main dipole configuration. The input signal (red) have amplitudes of $0.7 \mathrm{~A}, 6 \mathrm{~A}, 12 \mathrm{~A}, 18 \mathrm{~A}$ and $36 \mathrm{~A}$ reached within $10 \mu \mathrm{s}$.

extracted and plotted in Fig. 7.6 for all amplitudes of the step input. It can be seen that for a very small slew-rate $(0.78 \mathrm{~A} / 10 \mu \mathrm{s})$, the pole (at $1460 \mathrm{~Hz}$ ) has its corresponding zero at positive infinity meaning that increase in the controller gain in the feedback loop may cause this pole to move to the right-hand side of the complex plane resulting in the instability of the system around this pole's frequency (for explanation see sec. 2.8.2). The same is true for all other cases e.g. for the step of slew-rate of $6 \mathrm{~A} / 10 \mu \mathrm{s}$, the zero at $2.5 \mathrm{kHz}$ does not pair with the pole at $1.6 \mathrm{kHz}$ and this pole will also move to the right-half plane for higher controller gains which is a direct consequence of spatial model mismatch (conclusion drawn in Chapter 5), in the closed-loop response. On the other hand, the frequency of the dominant pole whose zero lies on the right hand side decreases for higher slew rate, meaning that higher the slew-rate, lower will be system's oscillation frequency. These measurements infer that in case of spatial model mismatch, the system can oscillate and can even be unstable at a particular frequency that depends upon the rate of change of corrector outputs.

\subsubsection{Full-system identification in open-loop}

The temporal-response measurement discussed in previous subsection for the corrector magnets and their associated components showed that the effective transfer functions for all horizontal corrector channels in SIS18 have slew-rate dependence. Moreover, it is found that two out of twelve corrector channels have different temporal responses. For the estimation of the controller parameters and to predict the stability of the closed-loop in the presence 


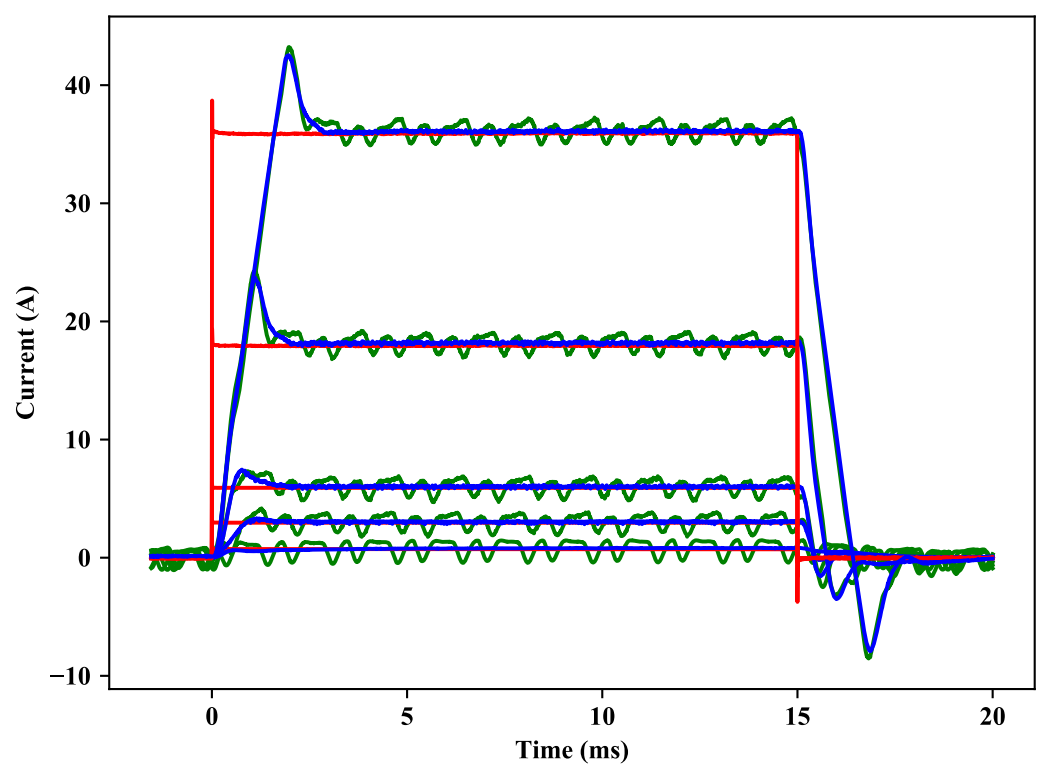

Figure 7.4 Pulse response of corrector magnet 11 along with its power converter and ACU for SIS18 (green) and SIS12 modes (blue) of main dipole configuration. The input signal (red) have amplitudes of $0.7 \mathrm{~A}, 6 \mathrm{~A}, 12 \mathrm{~A}, 18 \mathrm{~A}$ and $36 \mathrm{~A}$ reached within $10 \mu \mathrm{s}$.

of spatial model mismatch, system identification is further extended to the beam-based measurement for the full-loop, including all hardware components of the COFB system. The hardware included in the measurement are corrector magnets, their power converters and ACUs, vacuum chamber, beam, BPM hardware and Libera Hadron. In order to avoid integral action of the controller, $K_{p}$ and $K_{i}$ were set equal to 1 and 0 , respectively.

The feedback loop is interrupted at the point of controller output to achieve the open-loop configuration, in Libera Hadrons. For this purpose, a special arrangement is made using all three Libera Hadrons as shown in Fig. 7.7. In the presence of beam in SIS18, the COFB is made OFF in one of the Libera Hadrons and the sinusoidal excitation in the frequency range $100-1500 \mathrm{~Hz}$ from its synthetic waveform generator is split into two outputs (each of amplitude $0.7 \mathrm{~A}$ ) to connect to the oscilloscope as well for use as input to the system, for one corrector channel at a time. The response of the full-system in the form of the closed orbit oscillations is measured by all 12 BPMs and made available to all Libera Hadrons at the same time, in the form of global closed orbit. COFB is made ON at the second Libera whose corrector output is physically disconnected from the power converters and is recorded on the oscilloscope, as the system's output response. The oscilloscope is triggered at the Beam_ON event from the FTRN module of the third Libera. In this way, on one hand, the input and output are recorded in the same "corrector space" and on the other hand, almost all hardware are covered in the measurement.

In the full-system identification the corrector channels are found to have three different groups in terms of their temporal responses (Bode plots), having 9, 2 and 1 members, respectively. 

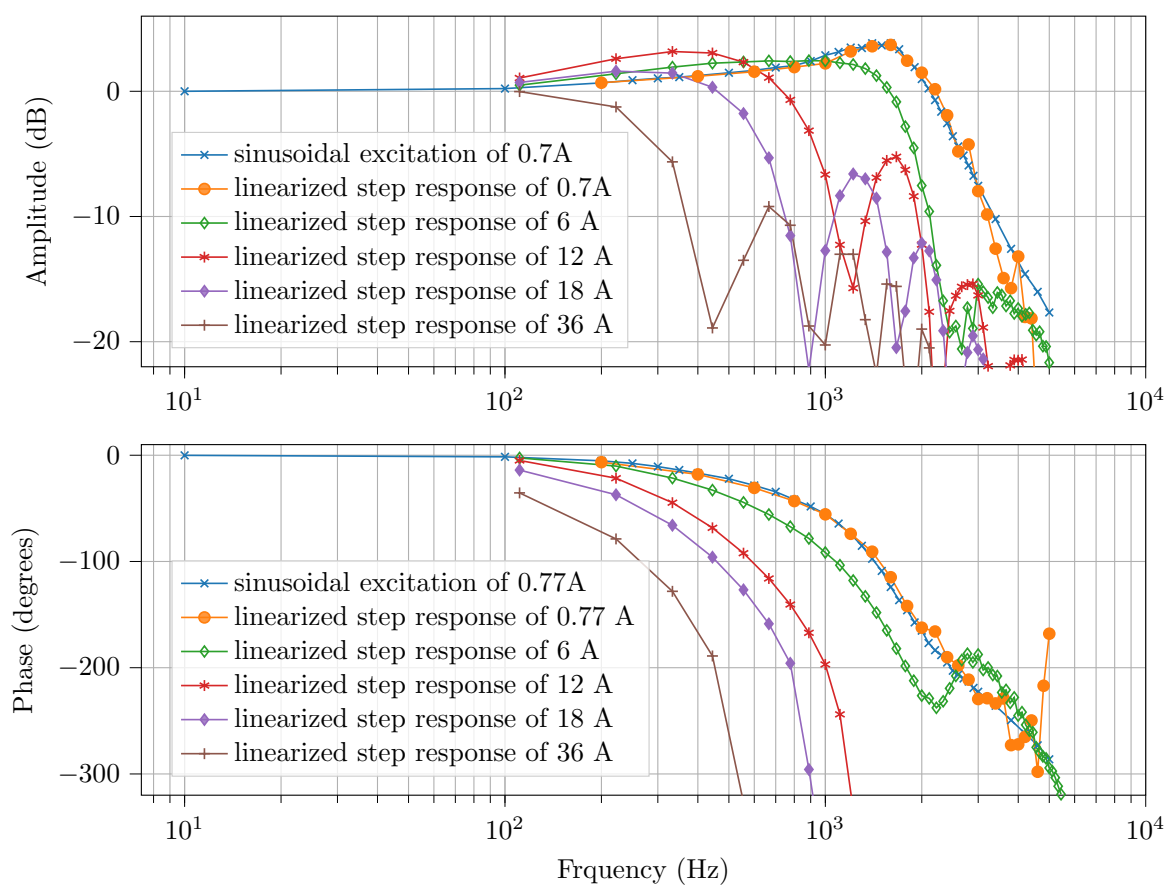

Figure 7.5 Bode plots for horizontal corrector 4 and its power converter in SIS18 mode extracted from its responses to the pulse inputs of variable heights per $10 \mu \mathrm{s}$.

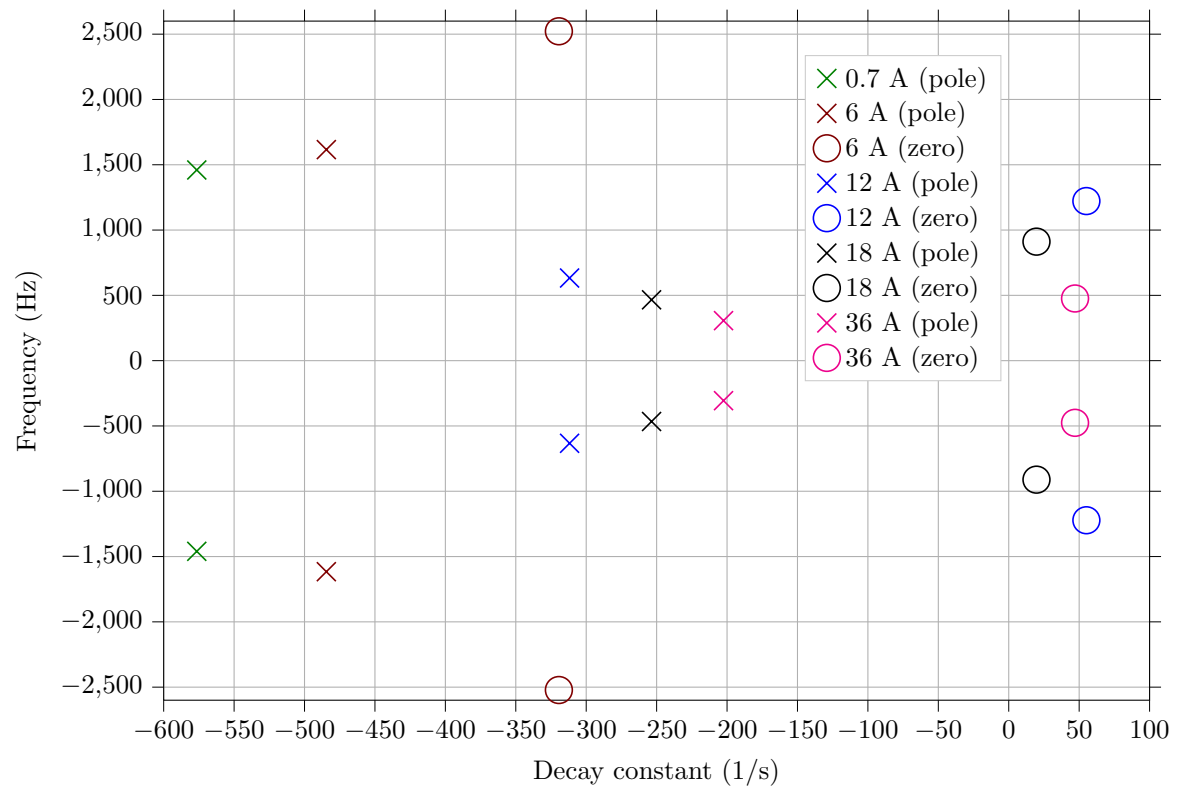

Figure 7.6 Dominant poles and zeros of estimated system models for various heights of step inputs (written in legend) per $10 \mu \mathrm{s}$.

Fig. 7.8 shows the Bode plots of 6 out of 9 similar corrector channels, while Fig. 7.9 shows three different behaviors, one representing each group. In comparison to subsystem identification, 


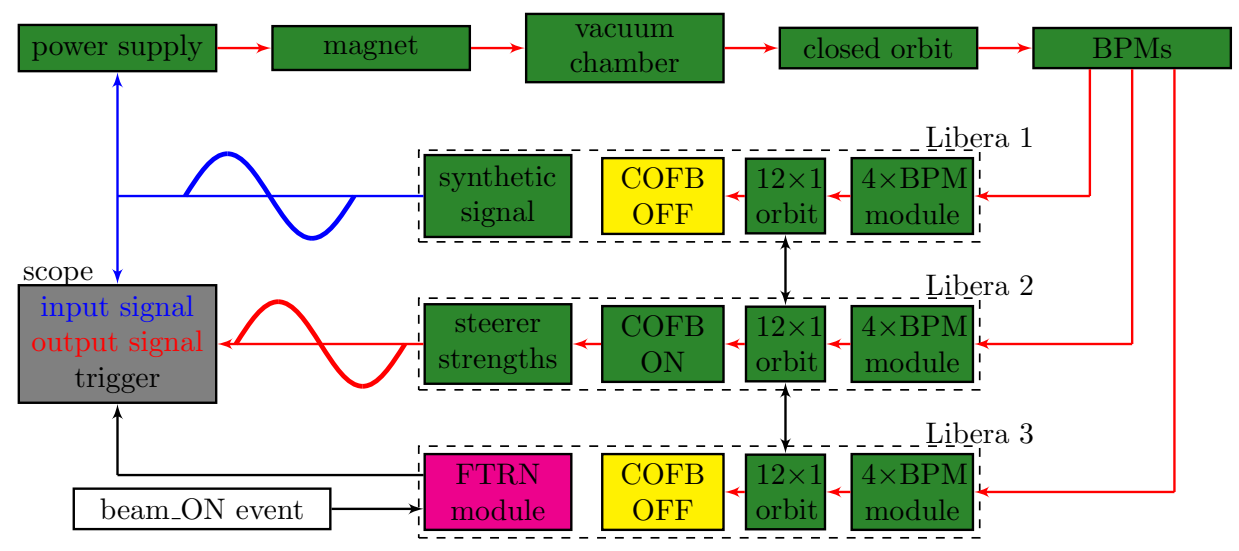

Figure 7.7 Schematic of the hardware arrangement for the open-loop system identification.

a new pole-zero pair appeared in the vicinity of 300 to $400 \mathrm{~Hz}$ for majority of the corrector channels in the full-system identification as predicted by the fitting in MATLAB, plotted in Fig. 7.10 only for corrector channel 4. For corrector channel 8, such a pole-zero pair is not detectable in the above mentioned frequency range (MATLAB fitting in Fig. 7.11). The group comprising of correctors marked as 10 and 11, which also showed a significantly different behavior in subsystem identification (Fig. 7.2), have a very small amplitude response (not shown) even for low frequencies (e.g. $100 \mathrm{~Hz}$ ) in comparison to all other corrector channels. The Bode Plot for corrector channel 11 in Fig. 7.9 is thus only a relative amplitude and phase advance compared to the their values at $100 \mathrm{~Hz}$. The positive phase advance at $300 \mathrm{~Hz}$ and consequently higher phase advance in comparison to other correctors, might be related to the fact that actual phase advance is more than $2 \pi$ radians and could not be measured in the comparison of peaks of input and output sinusoidal signals. In this way, the reliability of the measurement is only limited to 10 out of 12 corrector channels in the full-system identification. Moreover, these measurement are limited only to small input amplitude of 0.7 A as higher amplitude signals could not be applied for higher frequencies, due to the anti-oscillation interlocks of the power converters which resulted in the shutdown of the beam in SIS18. Also, pulse input excitation could also not be performed for the full-loop, in these measurements.

The transfer functions extracted from the fitting of Bode plots for correctors 4 and 8 are given in the zero-pole-gain form as,

$$
\begin{aligned}
& \text { fullsys } 4=e^{-0.00033 s} \frac{7702.7\left(s+1.071 \times 10^{4}\right)\left(s^{2}+649.6 s+4.78 \times 10^{6}\right)}{\left(s^{2}+562.6 s+4.176 \times 10^{6}\right)\left(s^{2}+9033 s+1.021 \times 10^{8}\right)} \\
& \text { fullsys } 8=e^{-0.00025 s} \frac{1165\left(s+6.345 \times 10^{4}\right)\left(s^{2}+1018 s+4.851 \times 10^{7}\right)}{\left(s^{2}+1289 s+4.657 \times 10^{7}\right)\left(s^{2}+9388 s+7.672 \times 10^{7}\right)}
\end{aligned}
$$

where fullsys represents the full-system transfer function whose important parameters are listed in table 7.3. There is a negative gain margin at frequency $1.3 \mathrm{kHz}$ for corrector 4 which is the representative of the majority of the corrector channels, meaning the system can be 

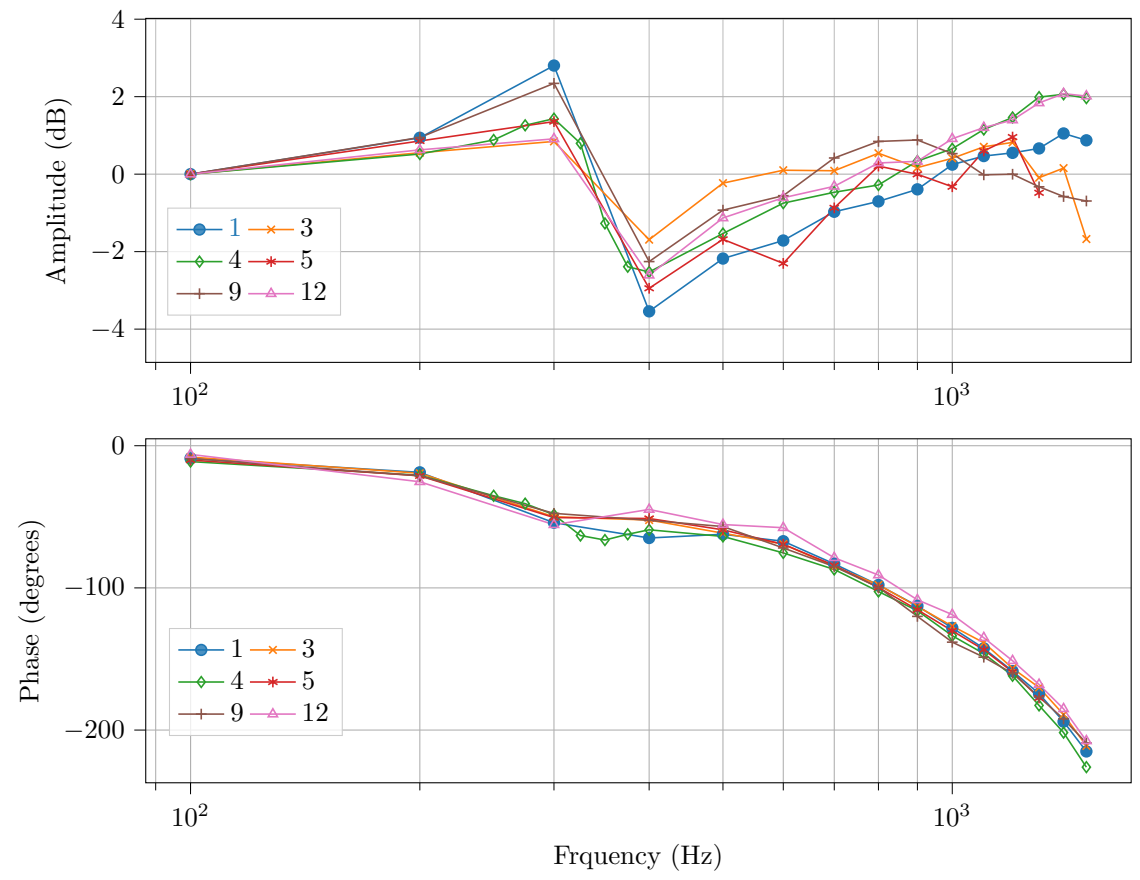

Figure 7.8 Measured Bode plots for the full-system for 6 of 9 similar horizontal corrector channels for sinusoidal excitation of $0.7 \mathrm{~A}$ amplitude.
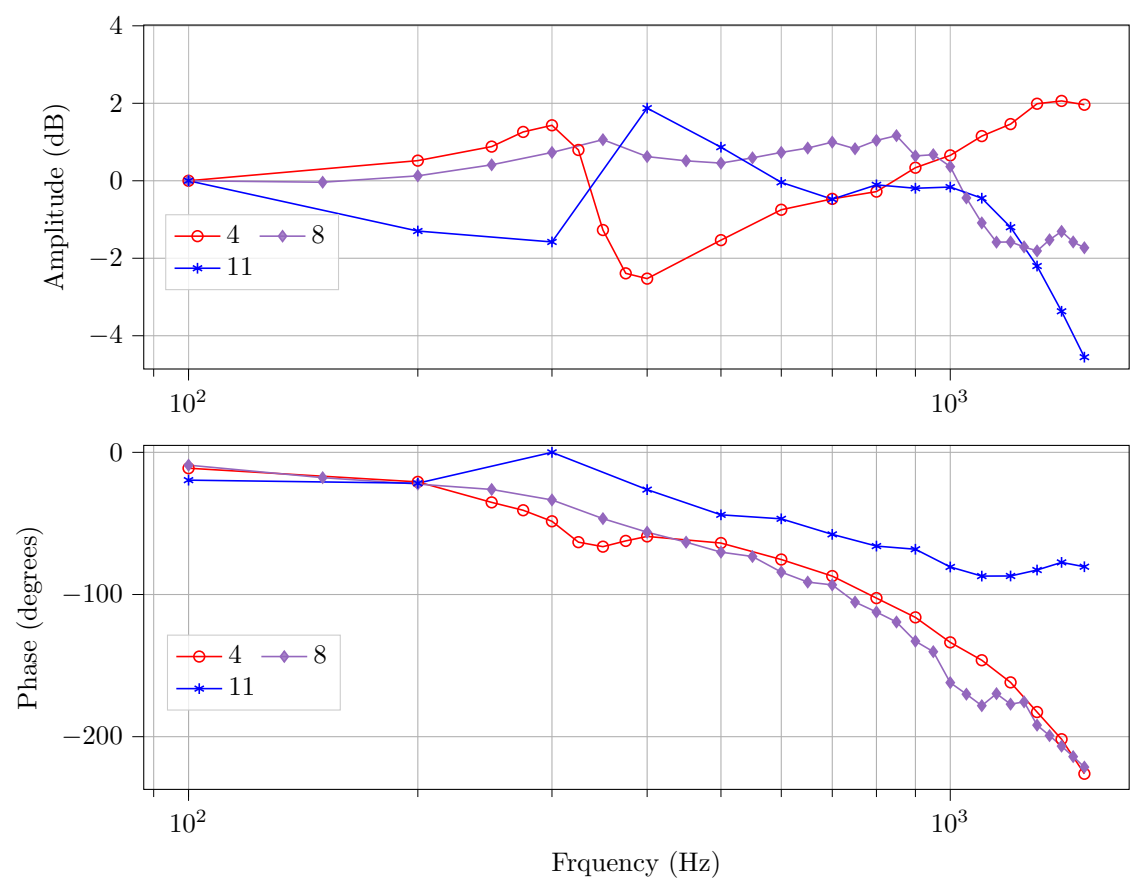

Figure 7.9 Measured Bode plots for the full-system for $4^{\text {th }}, 8^{\text {th }}$ and $11^{\text {th }}$ (similar to $10^{\text {th }}$ ) horizontal corrector channels for sinusoidal excitation of $0.7 \mathrm{~A}$ amplitude. 

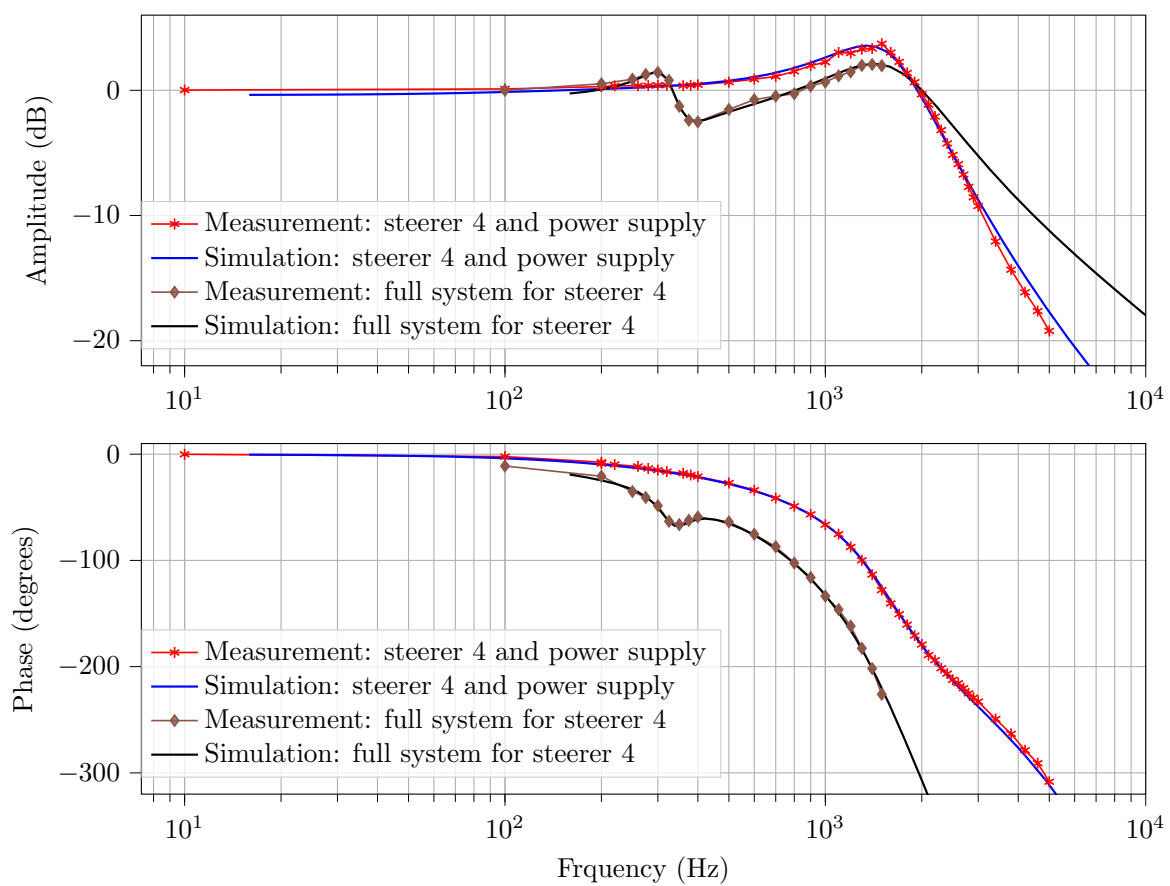

Figure 7.10 Measured and fitted Bode plot for the full-system for $4^{\text {th }}$ horizontal corrector channel for sinusoidal excitation of $0.7 \mathrm{~A}$ amplitude.
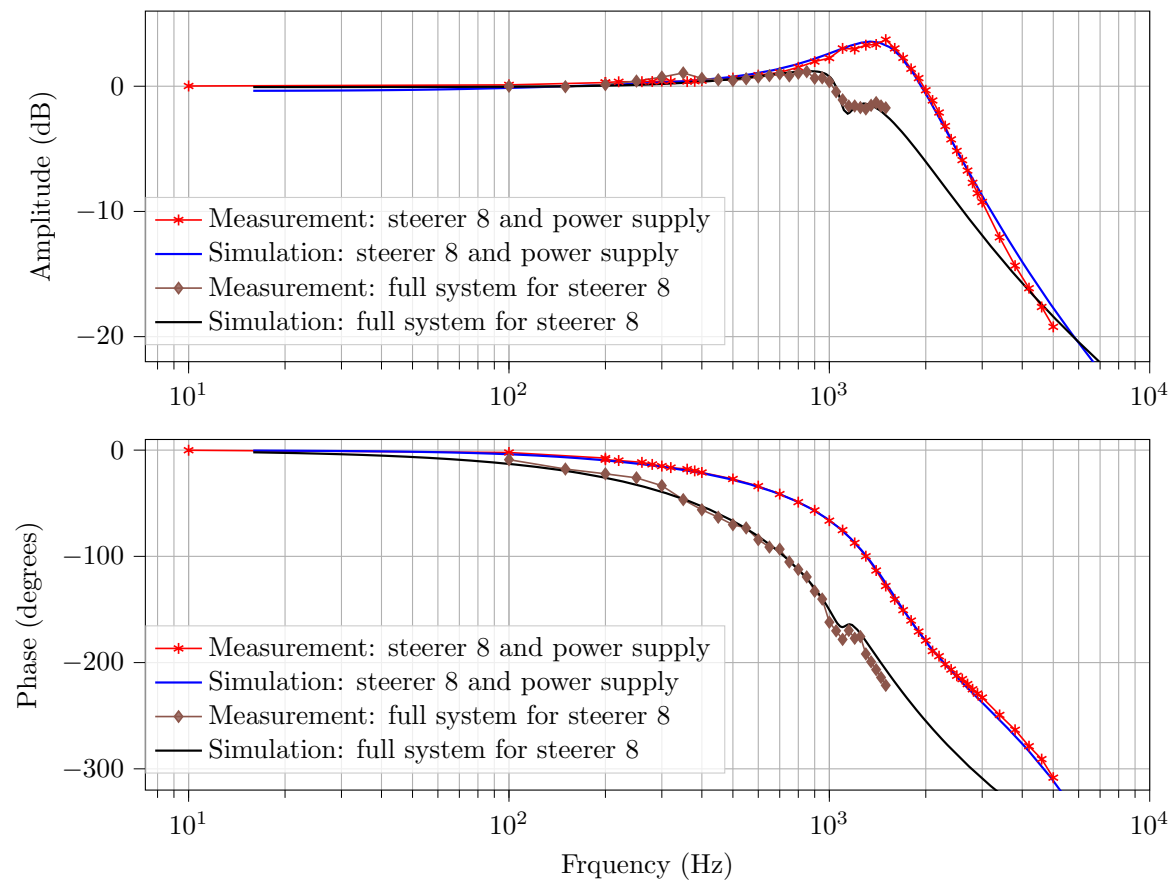

Figure 7.11 Measured and fitted Bode plot for the full-system for $8^{\text {th }}$ horizontal corrector channel for sinusoidal excitation of $0.7 \mathrm{~A}$ amplitude. 
Table 7.3 Parameters extracted from the full-system identification for the corrector channels 4 and 8. $p$ and $z$ represent the poles and zeros, respectively.

\begin{tabular}{llll}
\hline \hline Parameter & Unit & fullsys4 & fullsys8 \\
\hline \hline$p_{1}$ & $\mathrm{~Hz}$ & $-718.8+1438 \mathrm{j}$ & $-747.1+1177 \mathrm{j}$ \\
\hline$p_{2}$ & $\mathrm{~Hz}$ & $-718.8-1438 \mathrm{j}$ & $-747.1-1177 \mathrm{j}$ \\
\hline$p_{3}$ & $\mathrm{~Hz}$ & $-44.8+322.1 \mathrm{j}$ & $-102.5+1081 \mathrm{j}$ \\
\hline$p_{4}$ & $\mathrm{~Hz}$ & $-44.8-322.1 j$ & $-102.5-1081 \mathrm{j}$ \\
\hline$z_{1}$ & $\mathrm{~Hz}$ & $-1704.5+0 j$ & $-81.02+1106 \mathrm{j}$ \\
\hline$z_{2}$ & $\mathrm{~Hz}$ & $-51.7 .5+344.1 j$ & $-81.02-1106 \mathrm{j}$ \\
\hline$z_{3}$ & $\mathrm{~Hz}$ & $-51.7 .5-344.1 j$ & $-10100+0 \mathrm{j}$ \\
\hline delay & $\mu \mathrm{s}$ & 330 & 250 \\
\hline bandwidth & $\mathrm{Hz}$ & 2661 & 1608 \\
\hline gain margin & $\mathrm{dB}$ & -1.95 at $1.3 \mathrm{kHz}$ & 1.56 at $1.2 \mathrm{kHz}$ \\
\hline phase margin & $\mathrm{degrees}$ & 14.1 at $1.044 \mathrm{kHz}$ & 13.9 at $1.04 \mathrm{k} \mathrm{Hz}$ \\
\hline
\end{tabular}

unstable in case of higher controller gain which is the direct consequence of the spatial model mismatch. Looking at the imaginary parts of the poles and zeros, $p_{3}$ and $p_{4}$ form pairs with zeros $z_{2}$ and $z_{3}$ and these pairs lie on the left-half of the complex plane (i.e. real parts are negative). On the other hand, zeros corresponding to the poles having imaginary parts at $1.438 \mathrm{kHz}\left(p_{1}\right.$ and $\left.p_{2}\right)$ lie on the positive infinity, meaning that with higher controller gains, these poles can move to the right-hand side of the complex plane, resulting into the system instability at $1.438 \mathrm{kHz}$.

As, mentioned before, the current pole-zero arrangement is only for an excitation of very small amplitude of $0.78 \mathrm{~A} / 10 \mu \mathrm{s}$. In case of higher rate of change of corrector output, if the position of zero moves from left to the right of the complex plane for a pole of lower frequency, the system will oscillate at that pole frequency instead of $1.438 \mathrm{kHz}$ because of the finite location of its corresponding zero as compared to the infinity, in the presence of spatial model mismatch.

\subsection{Controller parameter estimation}

As mentioned in sec. 6.2.2, the controller implemented in the Libera Hadron for SIS18 COFB system is the Proportional-Integral (PI) type. The transfer function for a PI controller in 's' domain is given as,

$$
k(s)_{P I}=K_{p}+\frac{K_{i}}{s}
$$

where $K_{p}$ is unitless quantity and $K_{i}$ has the units of reciprocal of time $(1 / \mathrm{s})$. The PI controller parameters are estimated for the measured transfer functions for correctors 4 and 8 , in case of full-system identification using MATLAB class "pidtune" as,

$$
\mathrm{C}=\operatorname{pidtune}(\text { sys,type) }
$$


where 'sys' is the measured transfer function for the open-loop and type can be any combination of P, I and D. The type PI is used to estimate the $K_{p}$ and $K_{i}$ for corrector 4 and 8 and the values are given in Table. 7.4. It is important to mention that these controller parameters were regarded as an initial guess only, since the transfer functions measured as a result of full-system identification do not include the effect of slew-rate limited response.

Table 7.4 PI controller parameter estimation for measured transfer functions of corrector channels 4 and 8 .

\begin{tabular}{lll}
\hline \hline Transfer function & $K_{p}$ & $K_{i}$ \\
\hline \hline fullsys4 & 0.152 & $1340 / \mathrm{s}$ \\
\hline fullsys8 & 0.219 & $1940 / \mathrm{s}$ \\
\hline
\end{tabular}

\subsection{Orbit response matrix measurement}

Orbit response matrix (ORM) measurement is also a kind of system identification but in the spatial domain and is carried out throughout the ramps of different lengths (in time) using a fixed frequency excitation signal for each corrector channel, one by one. The response of the closed orbit on all BPMs to the excitation on one corrector magnet gives the elements of corresponding column of the ORM (Eq. 2.23). The excitation signals are sent to each corrector magnet using the synthetic wave generator of SER module of the Libera Hadron. The resultant closed orbit excitation are recorded with the help of already developed software named "TOPOS" [95] for all BPMs. The amplitude of the input excitation signal is chosen equivalent to a corrector kick of $1 \mathrm{mrad}$ at injection energy and the measured closed orbit excitation is normalized to the magnetic rigidity over each ramp. In this way, the amplitude of the closed orbit excitation is a direct measure of the elements of the ORM. Moreover, the intrinsic BPM offsets could also be eliminated from the measurement by subtracting the mean of the sinusoidal excitation of the closed orbit.

A typical response of the closed orbit over a ramp of maximum dipole field gradient of $1.4 \mathrm{~T} / \mathrm{s}$ to the excitation of $100 \mathrm{~Hz}$ on a single corrector, normalized to the beam rigidity is shown in Fig. 7.12 for 3 BPMs, as an example. The ORM elements are calculated from this data at the time intervals of $4 \mathrm{~ms}$ using a data length of $14 \mathrm{~ms}$ (more than one wave length) and the singular values of the corresponding ORMs are plotted as their direct signature in Fig. 7.13 . A clear variation of the singular values over the ramp can be seen from injection to extraction. Figure 7.14 shows the measured $\rho(\mathbf{M})$ over the ramp (defined in sec. 5.3) with respect to the ORM corresponding to the injection energy i.e. $\rho(\mathbf{M})=\rho\left(\mathbf{I}-\mathbf{R}(\mathbf{t}) \mathbf{R}_{\text {injection }}^{+}\right)$for MAD-X $\mathbf{R}_{\text {injection }}$ (blue) and measured $\mathbf{R}_{\text {injection (red). One can clearly see that MAD-X ORM has }}$ an intrinsic large model mismatch with respect to the measured ORM even at injection settings. The exact reason for such a large error in the MAD-X model of SIS18 is not known and further measurement-based studies are carried out in order to match the model with the measurement. On the other hand, the change in $\rho(\mathbf{M})$ with respect to injection settings for both cases is much larger up to $200 \mathrm{~ms}$ and $\rho(\mathbf{M})$ even crosses 1 between $77 \mathrm{~ms}$ and $92 \mathrm{~ms}$ 


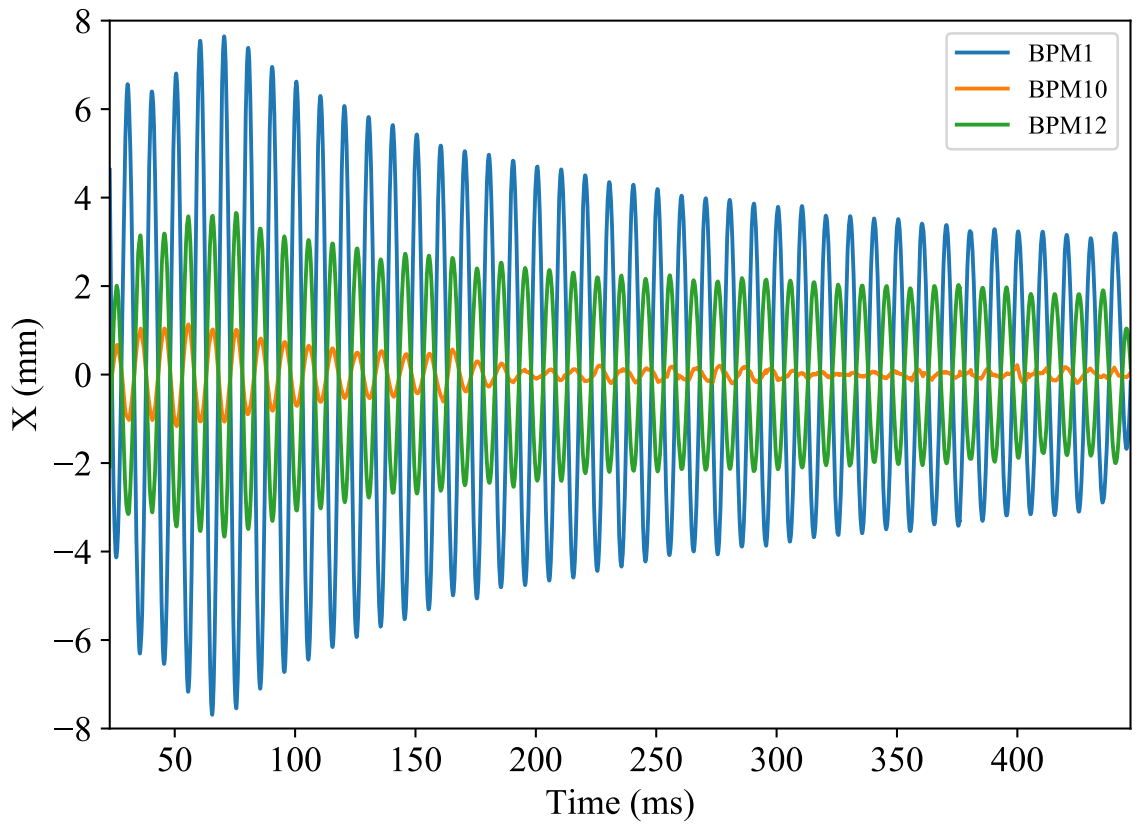

Figure 7.12 Example of closed orbit measured at 3 BPMs over the ramp as result of sinusoidal excitation of $100 \mathrm{~Hz}$ at corrector 1 for the ORM measurement (normalized with beam rigidity over the ramp).

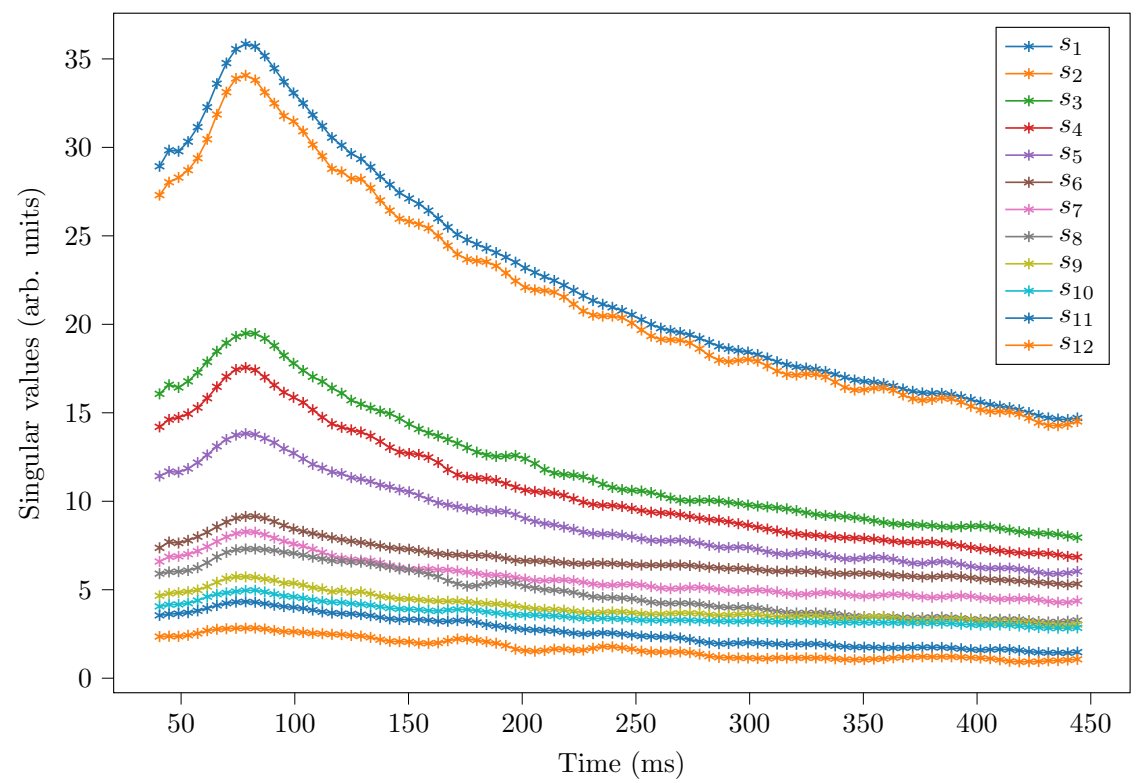

Figure 7.13 Measured singular values over a ramp of dipole field gradient of 1.4 $\mathrm{T} / \mathrm{s}$ at a step of $4 \mathrm{~ms}$ over a time window of $14 \mathrm{~ms}$ for each data point.

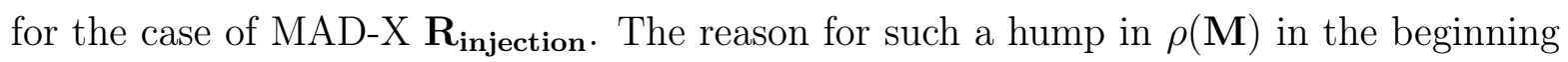




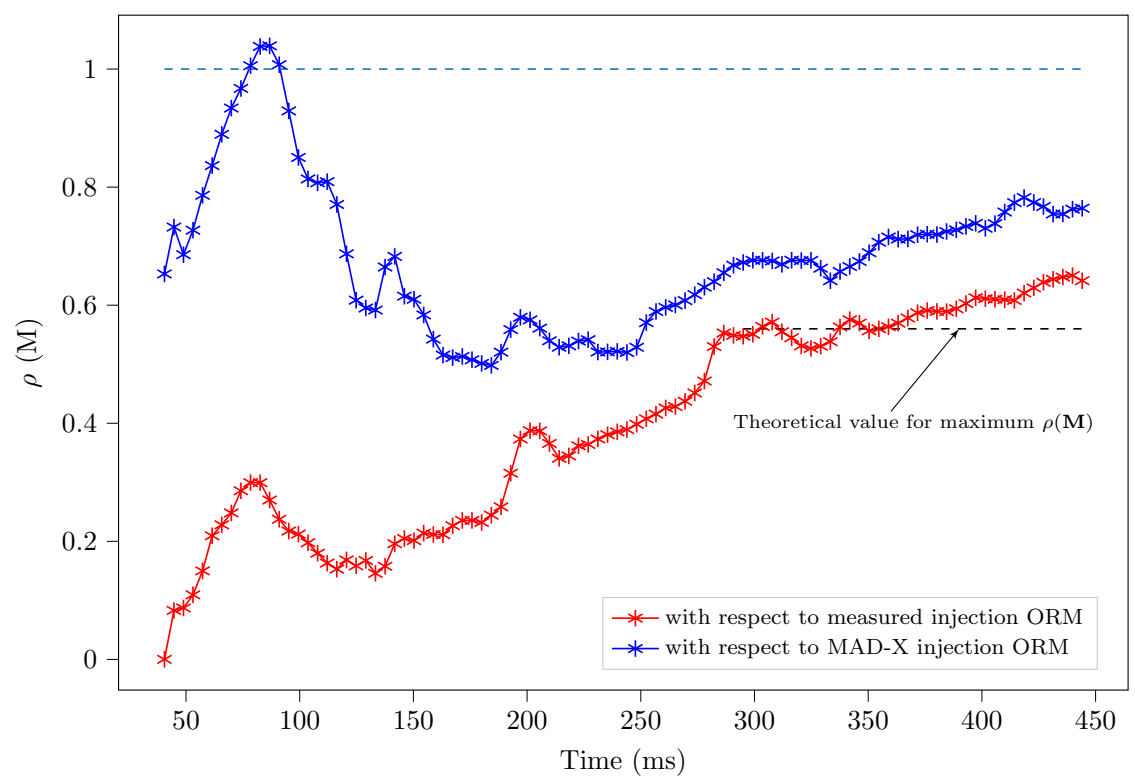

Figure 7.14 Measured spatil model mismatch over a ramp of dipole field gradient of $1.4 \mathrm{~T} / \mathrm{s}$ at a step of $4 \mathrm{~ms}$ over a time windows of $14 \mathrm{~ms}$.

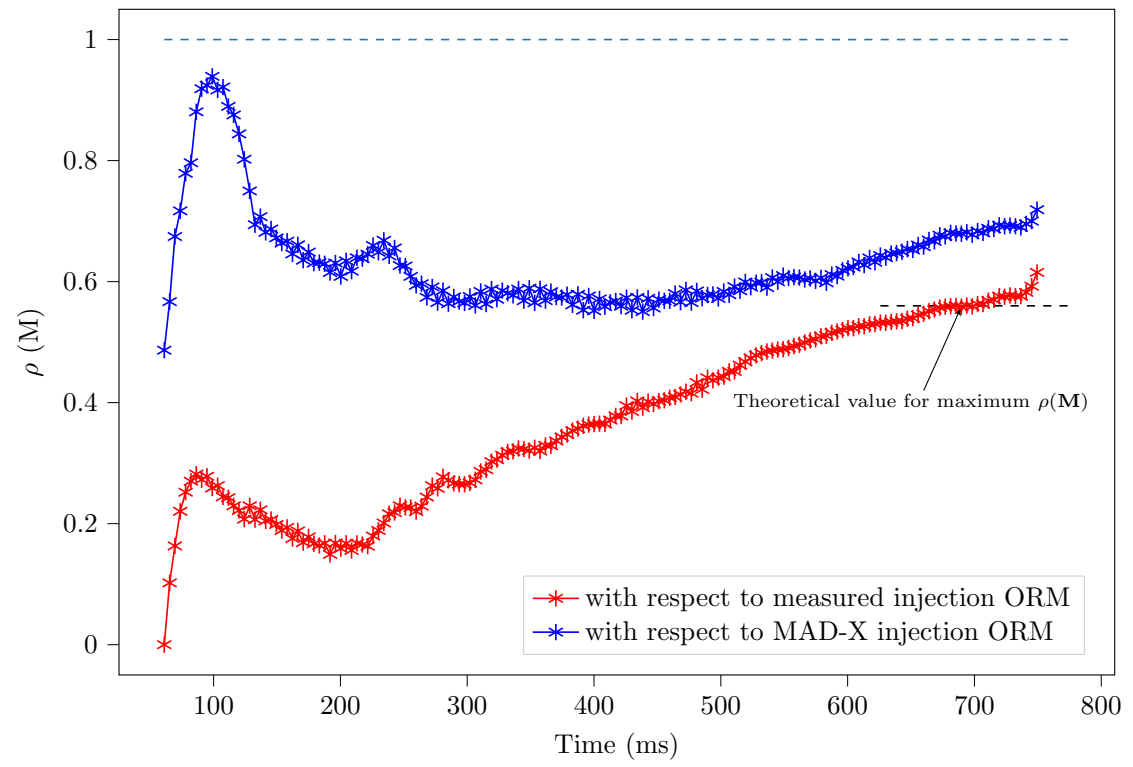

Figure 7.15 Measured spatial model mismatch over a ramp of dipole field gradient of $1.0 \mathrm{~T} / \mathrm{s}$ at a step of $4 \mathrm{~ms}$ over a time windows of $14 \mathrm{~ms}$.

of the ramp, can be associated with the quadrupole field errors caused by the quadrupole magnet power converters not being able to follow the fast gradient. A similar effect was already measured in the past on the coherent tune variation in the beginning of the ramp (Fig. 3.5). Fig. 7.15 shows the measured $\rho(\mathbf{M})$ over a relatively slower dipole field gradient i.e. 
1.0 T/s and one can see that the height of the hump in $\rho(\mathbf{M})$ in the beginning of the ramp is lower, as compared to the fast ramp. For both ramps, the maximum $\rho(\mathbf{M})$ at extraction with respect to measured injection ORM, is comparable to the theoretical values simulated in Fig. 5.8 for the horizontal plane and marked in both Figs. 7.14 and 7.15. The measurement of the $\rho(\mathbf{M})$ over the ramp is helpful for the prediction of the stability of the COFB system for the scenario of using a fixed ORM in the controller throughout the ramp (sec. 5.7).

\subsection{Closed-loop measurements}

SIS18 COFB hardware was first subjected to the basic beam-based capability tests in the closed-loop configuration during the beam time of February 2019. The tests comprised of communication between Libera hardware and the corrector power converters during the COFB being ON, the accuracy of the corrector strength calculation and the ability of the COFB system for the fast orbit correction over the ramp. Some of these tests performed without beam are already reported in Chapter 6. The beam-based tests have been helpful to make the FPGA programming of the hardware bug-free (via private communication with I-Tech Slovenia). A second more dedicated demonstration of the closed orbit correction and characterization of the COFB system in the closed-loop configuration was performed in November 2019 and the results are presented in the following subsections.

\subsubsection{On-ramp orbit correction}

\section{First-ever test}

Figure 7.16 (top) shows a perturbed closed orbit recorded on all BPMs over a ramp of $C^{6+}$ beam with number of particles of $1 \times 10^{8}$ from $11.38 \mathrm{MeV} / \mathrm{u}(\mathrm{B} \rho=0.974)$ to $300 \mathrm{MeV} / \mathrm{u}$ $(\mathrm{B} \rho=5.372)$. Besides the on-ramp beam position variation, the coupling transformers mentioned in subsection 7.1 also mixed some external noise to the BPM signal that appears as slow variation of beam position, particularly between 100 and $200 \mathrm{~ms}$ of the ramp. Figure 7.16 (bottom) shows the first ever effect of the fast COFB system recorded on the closed orbit of SIS18 in the horizontal plane throughout the ramp of beam settings mentioned above. COFB system was made ON upto $260 \mathrm{~ms}$ using a fixed MAD-X model of the ORM corresponding to the injection energy in the controller and for $K_{p}=0.12$ and $K_{i}=234 / \mathrm{s}$ (the measurement refers to the time before performing the system identification). It can be seen that the COFB system tried to bring the beam position on all BPMs closer to the golden orbit i.e. $0 \mathrm{~mm}$ but a non-zero residual was left over throughout the ramp (Fig. 7.16 bottom). Later on, it was found that 3 out of 12 correctors were disconnected from Libera hadron during that operation. The extent of orbit correction seen in Fig. 7.16 is achieved only by the remaining 9 correctors. These results anyhow demonstrated the capability of this COFB system for a fast orbit correction over the ramp as a first ever on-ramp implementation and is also reported in $[91]$. 

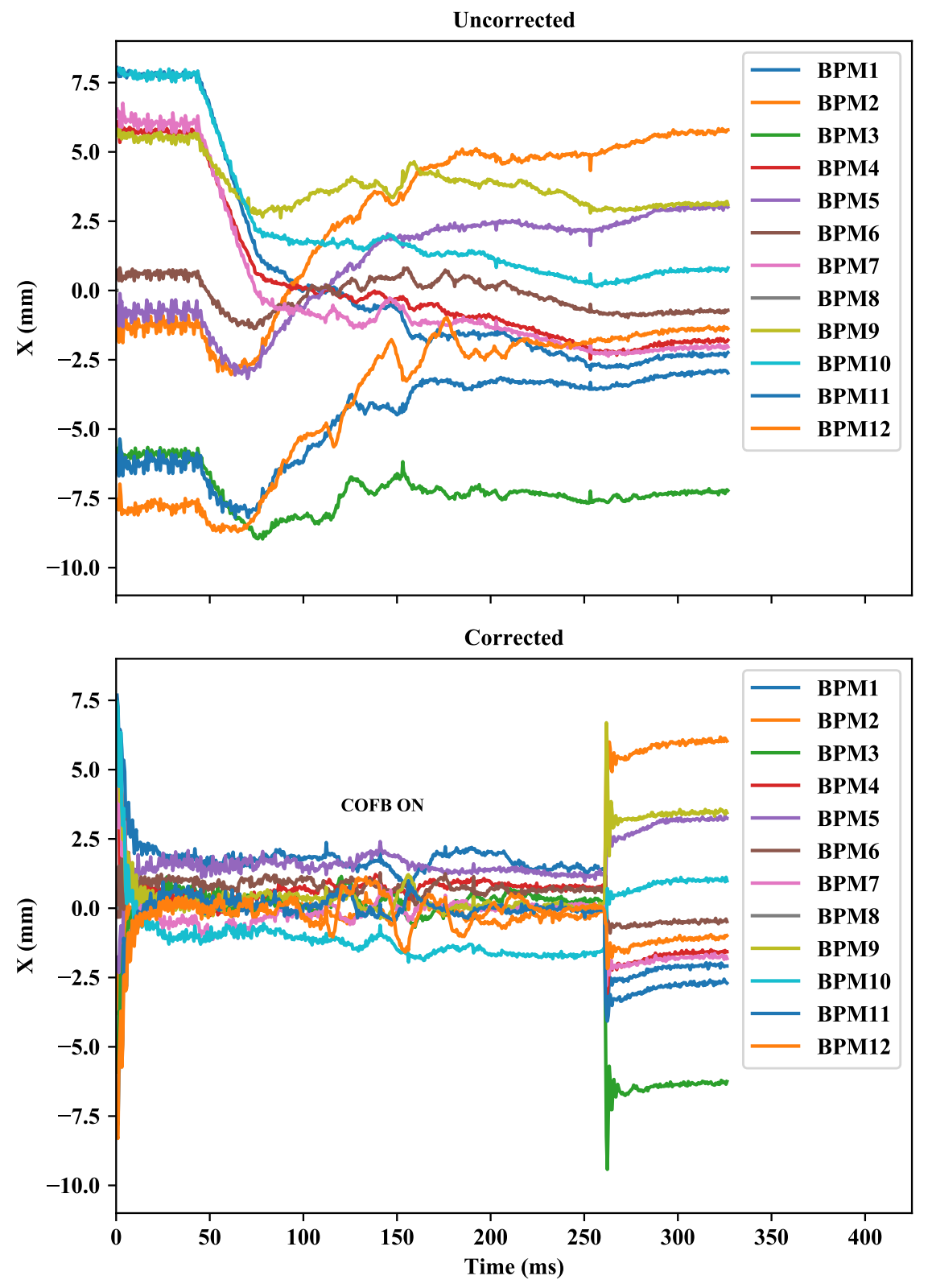

Figure 7.16 Orbit correction over the ramp in BPM space for the case of using MAD-X based model ORM corresponding to the injection energy in the controller. Measured beam position on all BPMs in SIS18 for uncorrected orbit (above) and for corrected orbit up to $260 \mathrm{~ms}$ (below).

\section{Orbit correction with MAD-X ORM}

A second more dedicated demonstration of the orbit correction in SIS18 is made in November 2019 where all the correctors were connected to the Libera hadron and the system was 

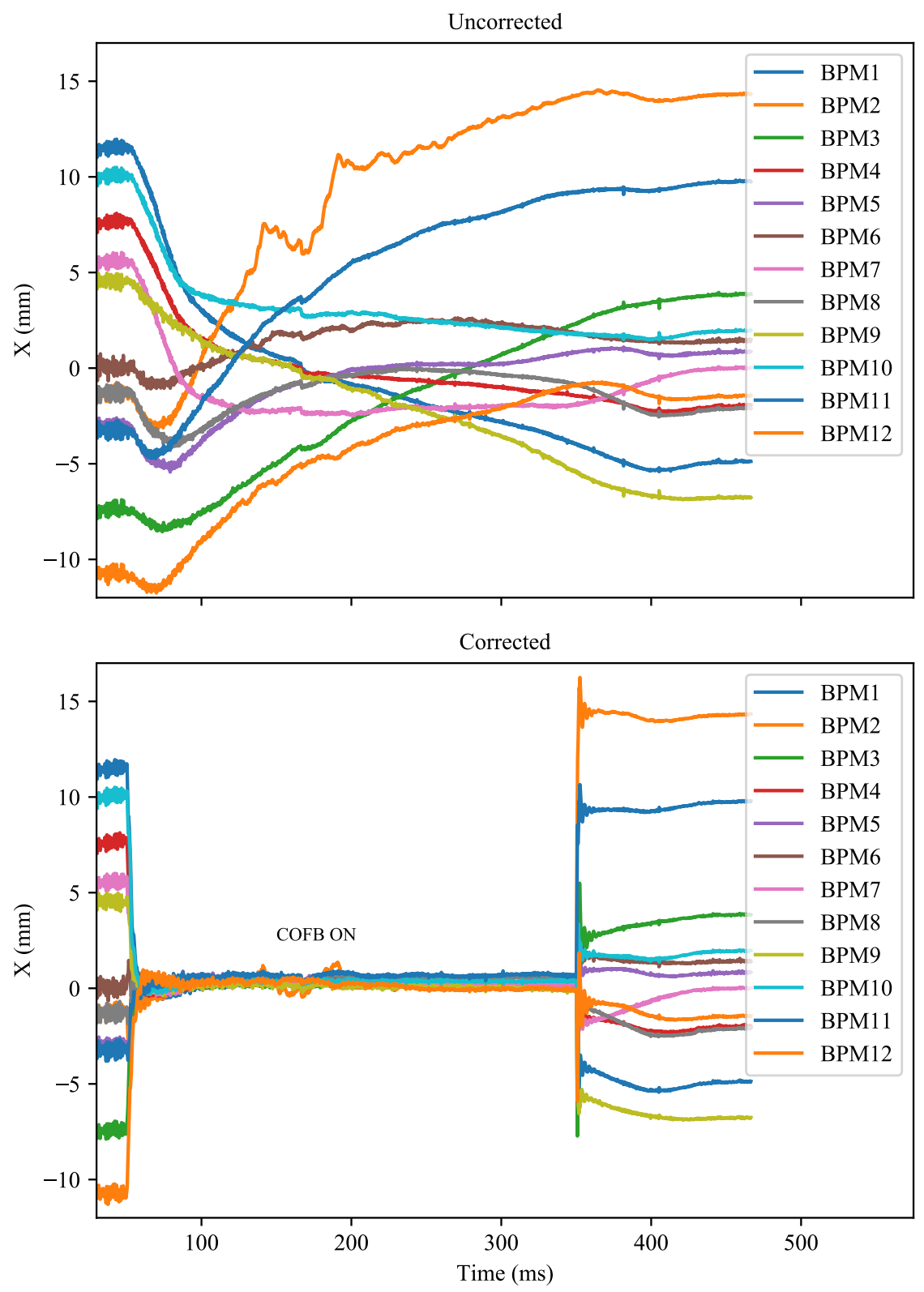

Figure 7.17 Orbit correction over the ramp in BPM space for the case of using MAD-X based model ORM corresponding to the injection energy in the controller. Measured beam position on all BPMs in SIS18 for uncorrected orbit (above) and for corrected orbit from $40 \mathrm{~ms}$ to $350 \mathrm{~ms}$ (below) using $K_{p}=0.046$ and $K_{i}=234 / \mathrm{s}$.

equipped with an improved bug-free version of FPGA programming of the Libera Hadron. A beam of $A r^{18+}$ is accelerated from $11.38 \mathrm{MeV} / \mathrm{u}$ to $300 \mathrm{MeV} / \mathrm{u}$ within an acceleration cycle of $450 \mathrm{~ms}$ and the COFB is made ON at $40 \mathrm{~ms}$ and is made OFF at $350 \mathrm{~ms}$, with respect to the ramp start. Also in this case, the COFB is run in a fixed-ORM mode with the MAD-X 


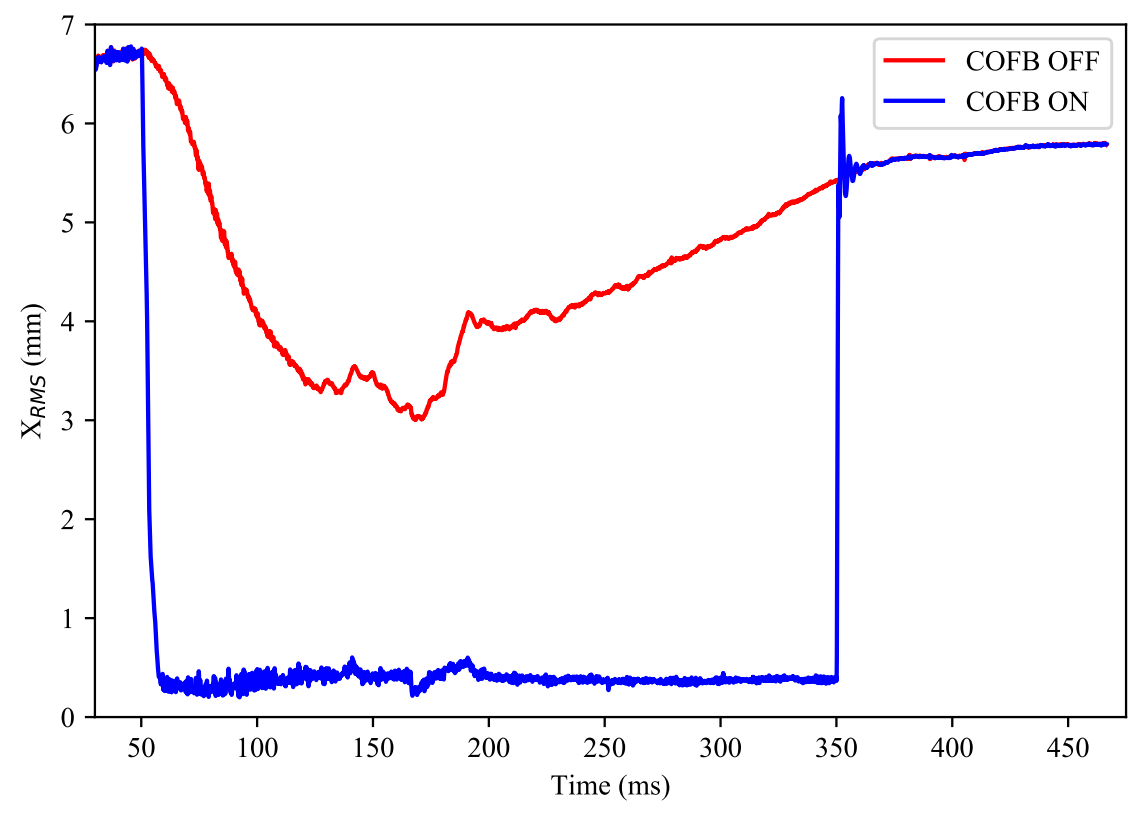

Figure 7.18 RMS calculated for uncorrected and corrected closed orbits of Fig. 7.17 where $K_{p}=0.046$ and $K_{i}=234 / s$ for the orbit correction.

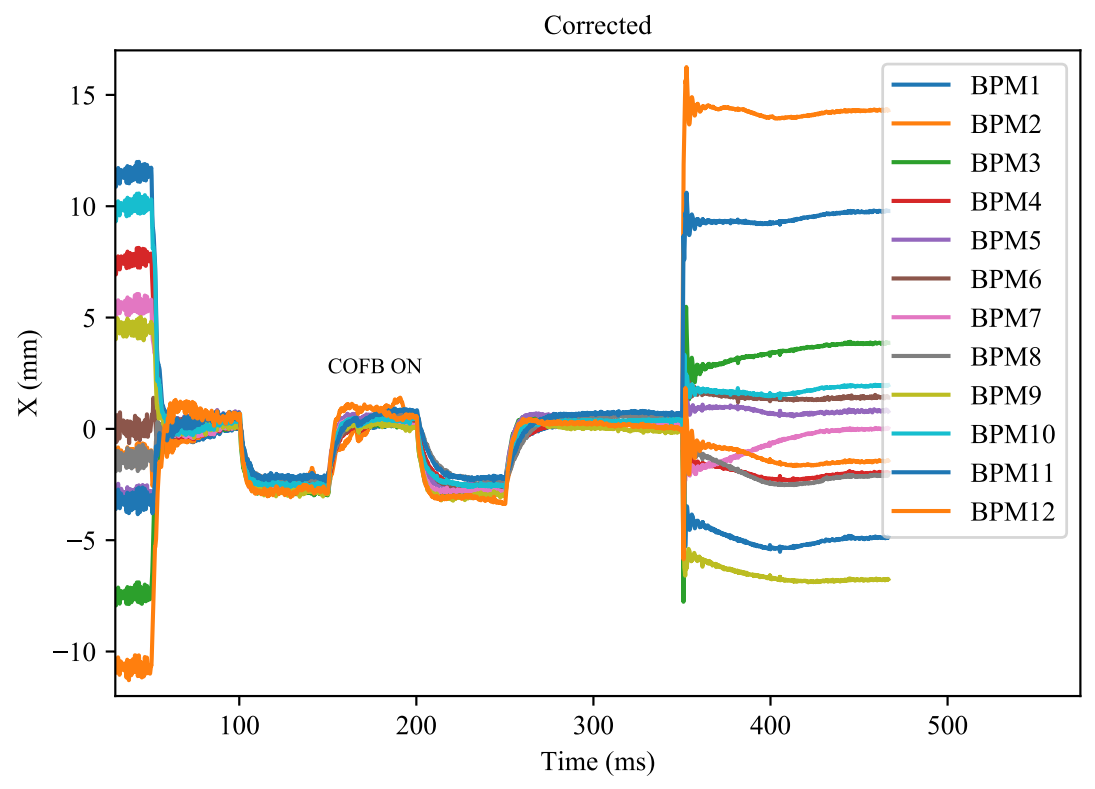

Figure 7.19 Golden orbit manipulation during the orbit correction over the ramp. Measured beam position on all BPMs in SIS18 for corrected orbit from $40 \mathrm{~ms}$ to $350 \mathrm{~ms}$ using $K_{p}=0.046$ and $K_{i}=234 / \mathrm{s}$. The MAD-X based model ORM corresponding to the injection settings is used in the controller. 


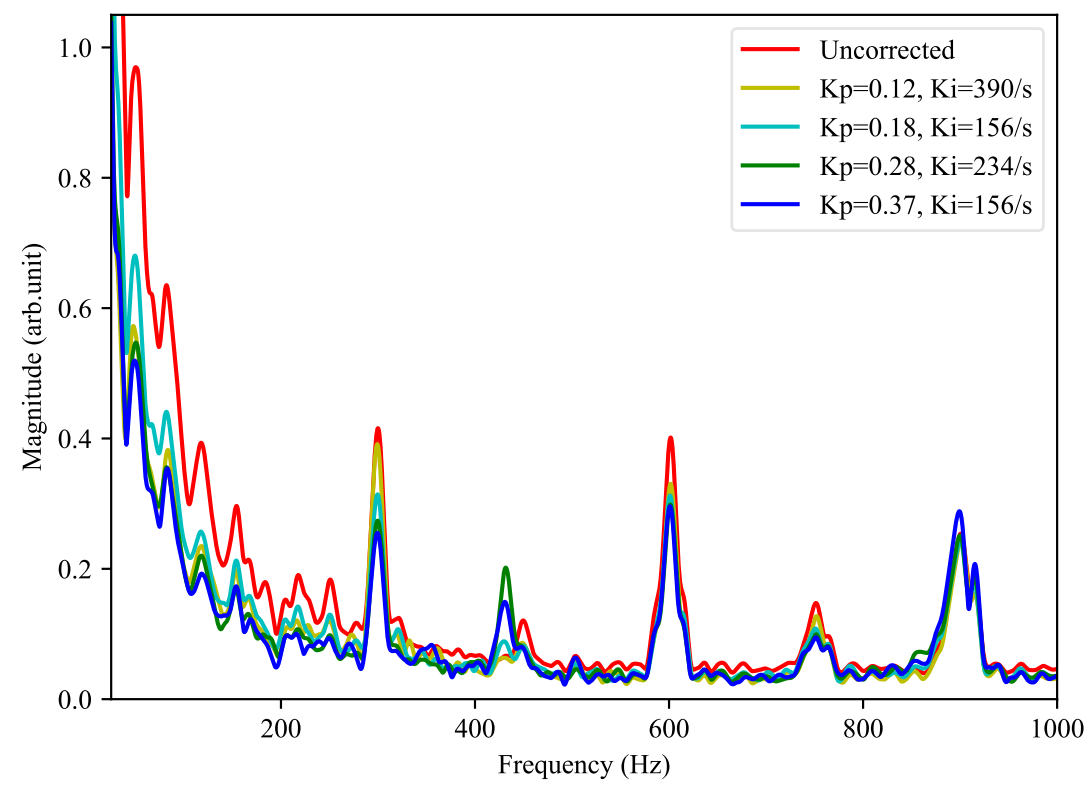

Figure 7.20 Orbit correction for SIS18 in frequency domain (average of the DFT of position data from all BPMs) for various controller gains for the case of using MAD-X based model ORM corresponding to the injection settings in the controller.

ORM corresponding to the injection settings in the controller. Figure 7.17 shows the beam position on all $12 \mathrm{BPMs}$ before and after orbit correction for with $K_{p}=0.046$ and $K_{i}=234 / \mathrm{s}$. The effect of transformer coupling is more prominent for BPM2 in these measurements. Initially, the controller gains are kept very small as compared to the estimated gains in order to demonstrate the orbit correction only for the DC component (zero frequency) of the closed orbit motion while high frequency correction is ignored so far. The achieved orbit correction is very promising in terms of the RMS of the DC component below $1 \mathrm{~mm}$ throughout the ramp during the COFB being ON, as shown in Fig. 7.18. The comparison of top and bottom subplots of Fig. 7.17 shows that the closed orbit returns to its uncorrected position at 350 $\mathrm{ms}$ as the COFB system is switched OFF. These results show a very good tracking ability of the COFB system to the set point which is further demonstrated in Fig. 7.19 with an artificially varied reference orbit over the ramp and the COFB system followed it. In order to perform high frequency orbit correction, the controller gains are increased gradually towards the estimated values given in Table. 7.4 as the estimated $K_{i}$ value resulted in the closed orbit oscillations and consequently beam loss. The reason for such an instability is discussed in detail in next section. The frequency spectra of the uncorrected as well as corrected closed orbit for a set of controller gains are shown in Figure 7.20. Each curve is the average of the DFT of all 12 BPMs data for each set of controller gains. The possible source of peaks at $150 \mathrm{~Hz}$ and its higher harmonics is already discussed in sec. 3.2. One can see that orbit correction up to $300 \mathrm{~Hz}$ has been achieved for all controller gains (the best for $K_{p}=0.37$ and $\left.K_{i}=156 / s\right)$. Moreover, the DC value of DFT of corrected data is much smaller as compared 
to the uncorrected data in proportion to the $X_{R M S}$ but not shown in Fig. 7.20 due to very large amplitude for uncorrected orbit, as compared to other high frequency peaks.

\section{Orbit correction with measured ORM}

Orbit correction is further performed for another acceleration cycle of different machine settings i.e. dipole field gradient of $1.0 \mathrm{~T} / \mathrm{s}$, ramp length of $600 \mathrm{~ms}$, particle beam of $U^{28+}$ and beam rigidity changing from 3.6 Tm to $18.2 \mathrm{Tm}$, over the ramp. The extraction energy was $300 \mathrm{MeV} / \mathrm{u}$ and number of particles were $1 \times 10^{8}$, as before. COFB system is run in the same fixed-ORM mode but using the measured ORM corresponding to injection settings instead of MAD-X model, hence the model mismatch over the ramp is given by the red curve of Fig. 7.15. For this measurement, BPM2 had to be excluded from the input array of closed orbit to the feedback controller since it had strong influence of the coupling transformer mentioned in sec. 7.1. Such an external noise signal, when fed to the COFB system, can be another source of closed orbit instability as the integral part of feedback system would keep on building up the corrector current to compensate a BPM signal which is not changing as the result of feedback action.

For the case of using measured ORM in the controller, the PI gains are increased gradually and closed orbit instability is not observed even for the values larger than estimated in Table. 7.4. Figure 7.21 shows the BPM data of 9 BPMs before (above) and after (below) making the COFB system $\mathrm{ON}$ for $K_{p}=0.37$ and $K_{i}=2343 / s$. The corresponding frequency spectrum of uncorrected and corrected closed orbit is shown in Fig. 7.22 where it is obvious that the disturbance rejection could be achieved up to $600 \mathrm{~Hz}$. An enhancement in the lower frequency side of spectrum is most probably the consequence of the external noise signal being fed to the feedback loop in the presence of very high integral gain, by the other BPMs. A comparison of Fig. 7.20 and 7.22 clearly proves the conclusion drawn in Chapter 5 that the achievable bandwidth of disturbance rejection is reduced with the spatial model mismatch.

\subsubsection{Spatial model mismatch and COFB system stability}

In the previous subsection, following observations have been made during the on-ramp orbit correction,

- In case of using fixed MAD-X ORM in the controller throughout the ramp, which corresponds to the large spatial model mismatch (blue curves in Figs. 7.16 and 7.17) the controller gains (particularly $K_{i}$ ) could only be applied to certain fraction of the estimated values and orbit correction could only be achieved up to $300 \mathrm{~Hz}$.

- In case of using measured ORM, the controller gains could even be applied higher than the estimated values, and orbit correction could be achieved up to $600 \mathrm{~Hz}$.

Both of the above experimental observations confirm a strong influence of the spatial model mismatch on the performance of the COFB system. In this subsection, the effect of such a model mismatch is further investigated experimentally for the SIS18 COFB system, in the light of and as a confirmation of theoretical and simulation results worked out in chapter 5. In sec. 5.7, it is shown that larger $\rho(\mathbf{M})$ can make the COFB system unstable at the frequency 

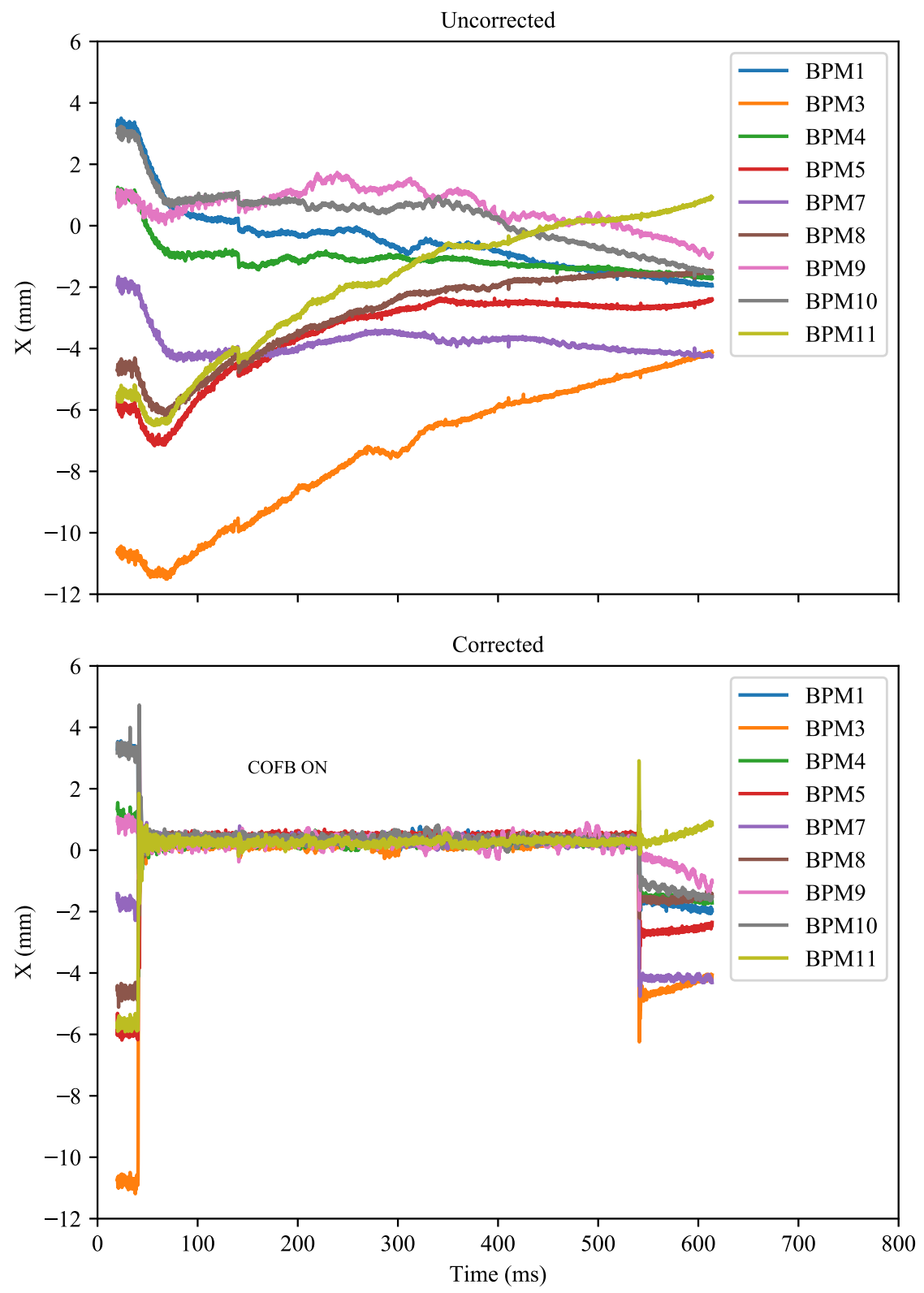

Figure 7.21 Orbit correction over the ramp in BPM space. Measured beam position on all BPMs in SIS18 for uncorrected orbit (above) and for corrected orbit from 40 ms to $540 \mathrm{~ms}$ (below) for the case of using measured ORM corresponding to the injection settings and controller gains of $K_{p}=0.37$ and $K_{i}=2343 / \mathrm{s}$.

of the dominant pole of the open-loop system whose corresponding open-loop zero is on the right-hand side of the complex plane. The pole movement as a function of $\rho(\mathbf{M})$ for a first-order system is shown as an example, by the simulations in Fig. 5.18. Furthermore, the scaling (for decrease) of the controller gains with $\rho(\mathbf{M})$ required to avoid instability of the 


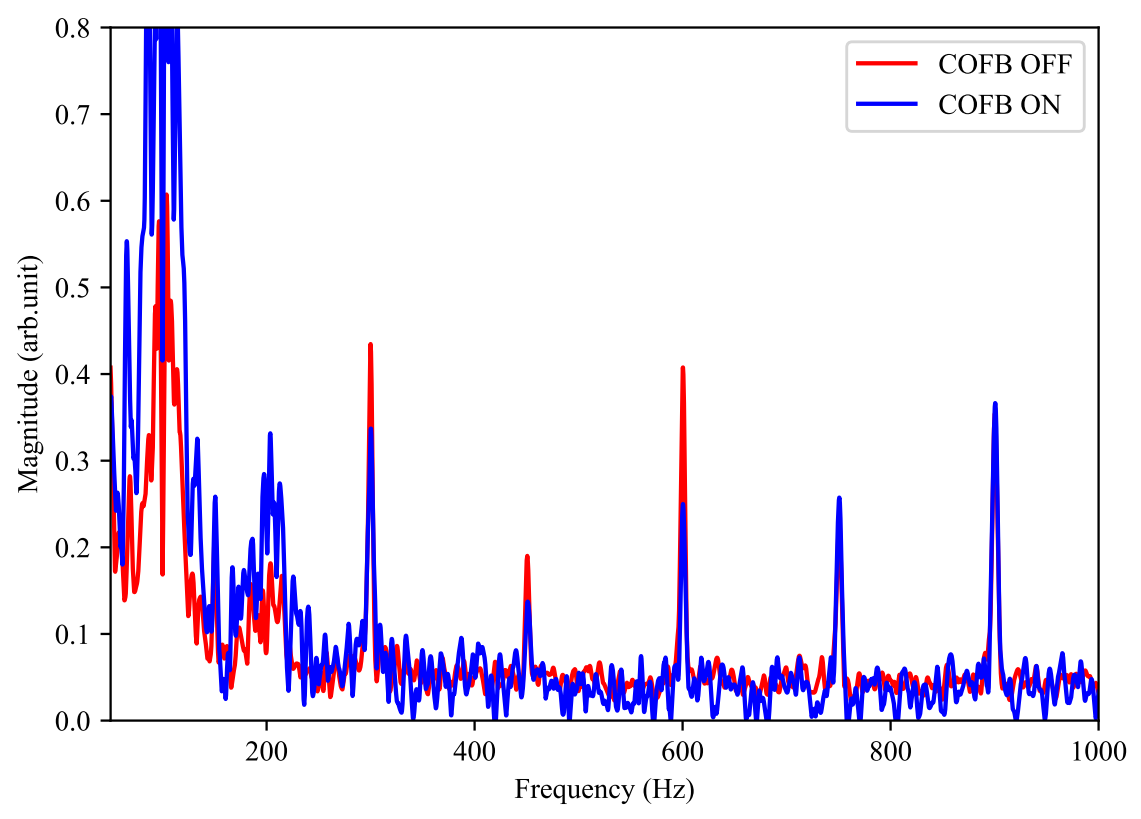

Figure 7.22 Average of the DFT of position data from 9 BPMs (excluding BPMs 2, 6 and 12) for COFB OFF (red) and COFB ON (blue) with $K_{p}=0.37$ and $K_{i}=2343 / s$, using measured ORM corresponding to the injection settings in the controller. For explanation of low frequency enhancement, please see text.

COFB system is also described in Eq. 5.38.

In order to achieve the $\rho(\mathbf{M})$-dependent instability of the SIS18 COFB system, the ramp of $A r^{18+}$ from $11.38 \mathrm{MeV} / \mathrm{u}$ to $300 \mathrm{MeV} / \mathrm{u}$ is used for orbit correction with the MAD-X ORM of injection settings for which $\rho(\mathbf{M})$ follow the blue curve of Fig. 7.14 and the best disturbance rejection is achieved for controller gains of $K_{p}=0.37$ and $K_{i}=156 / s$, previously (Fig. 7.20). $\mathrm{COFB}$ is made $\mathrm{ON}$ at $40 \mathrm{~ms}$ and the proportional gain is fixed to this value while integral gain is increased and resultant beam position on BPM12 is shown in Fig. 7.23 for a number of integral gains ( $K_{p}$ and $K_{i}$ are both constant over one ramp). The frequency spectrum of the BPM data is shown in Fig. 7.24. One can see that with the increase of controller gain $K_{i}$, the closed orbit starts oscillations with following two interesting facts,

- The closed orbit oscillation occurs only over a specific time window of the ramp.

- The frequency of oscillations i.e. $\approx 335 \mathrm{~Hz}$, does not exist in the uncorrected closed orbit as can be seen in the red curve in Fig. 7.22. Anyhow, this frequency of oscillations is closer to the one of the open-loop pole frequency of $322 \mathrm{~Hz}$ named as $p_{3}$ in Table 7.3 .

The existence of oscillations only for a specific time window for higher controller gain can be explained with the help of $\rho(\mathbf{M})$ curve plotted over the BPM data in Fig. 7.23. The amplitude of closed orbit oscillations first increases and then decreases (as does $\rho(\mathbf{M})$ ) which gives a strong hint on the movement of the dominant pole over the ramp, from left- to right-half 


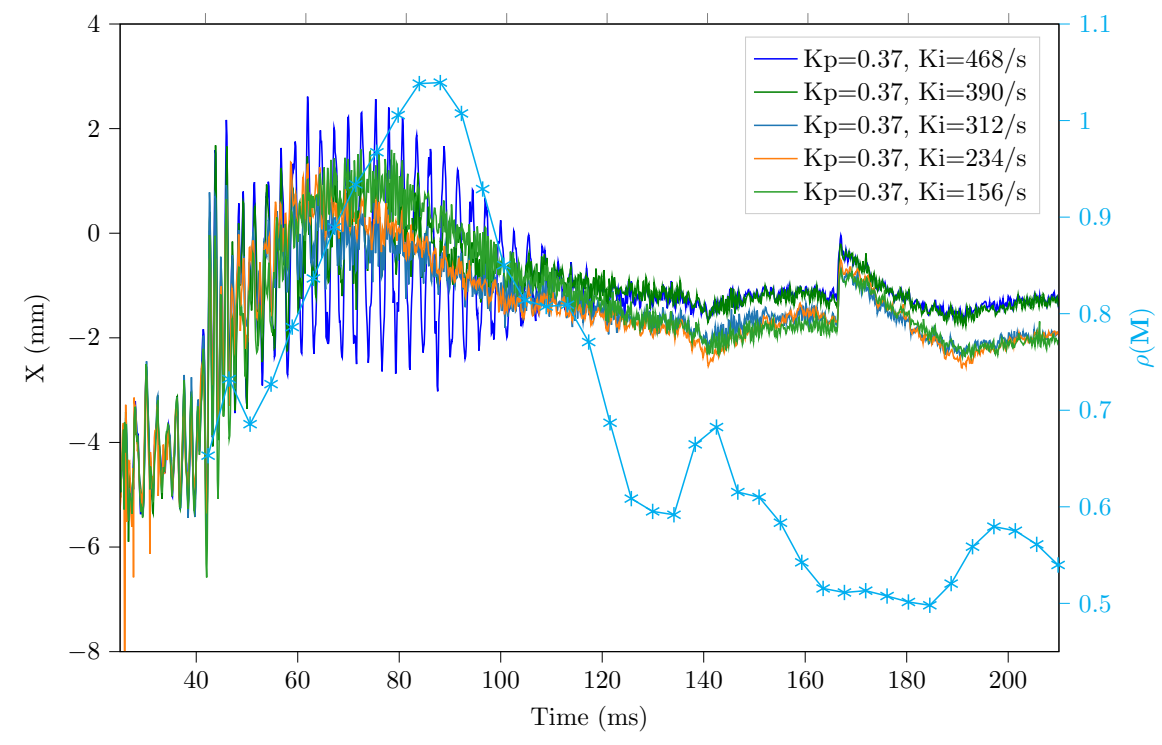

Figure 7.23 Closed orbit excitation for higher controller gains for the case of using MAD-X based model ORM corresponding to the injection settings in the controller. The induced oscillations are in the region of maximum $\rho(\mathbf{M})$.

of the complex plane and then back to the left-half, following the pattern of $\rho(\mathbf{M})$. On the other hand, no oscillations for smaller controller gains is also consistent with the theoretical conclusions made in sec. 5.7 that for larger $\rho(\mathbf{M})$, the controller gain needs to be reduced to avoid oscillations and consequently instability. In case of small $\rho(\mathbf{M})$, even much higher controller gains $\left(K_{p}=0.37\right.$ and $\left.K i=2343 / s\right)$ do not cause any closed orbit oscillations at this frequency, as evident from Fig. 7.22 where the $\rho(\mathbf{M})$ follows the red curve of Fig. 7.15 over the ramp.

The measured frequency of closed orbit oscillation is an apparent contradiction with the prediction of the open-loop system identification. As mentioned before, there exists a conjugate pair of open-loop poles $p_{3}$ and $p_{4}$ in Table 7.3 at frequency of $322 \mathrm{~Hz}$ and the frequency of closed-loop oscillation being $335 \mathrm{~Hz}$ shows that the open-loop pole sensitive to the spatial model mismatch must be $p_{3}$ or $p_{4}$. But the zeros corresponding to these poles lie on the left-hand side of the complex plane (negative real parts) which means that movement of these poles towards their zeros for larger $\rho(\mathbf{M})$ will not let these poles to appear on the right-hand side of the complex plane and hence, the closed-loop system should not oscillate and diverge at these pole frequencies. Anyhow, it is important to recall that the open-loop poles and zeros given in Table 7.3 are measured as a result of system identification performed with a sinusoidal signal much below the slew-rate limitation. It is observed in the subsystem identification in sec. 7.2 .1 that the open-loop zeros corresponding to the poles of lower frequency can be shifted to the right-hand side of complex plane for higher slew rates of input signal as shown in Figs. 7.5 and 7.6. Based upon above information, it is concluded that,

- The instability observed in the form of closed orbit oscillations for higher controller 


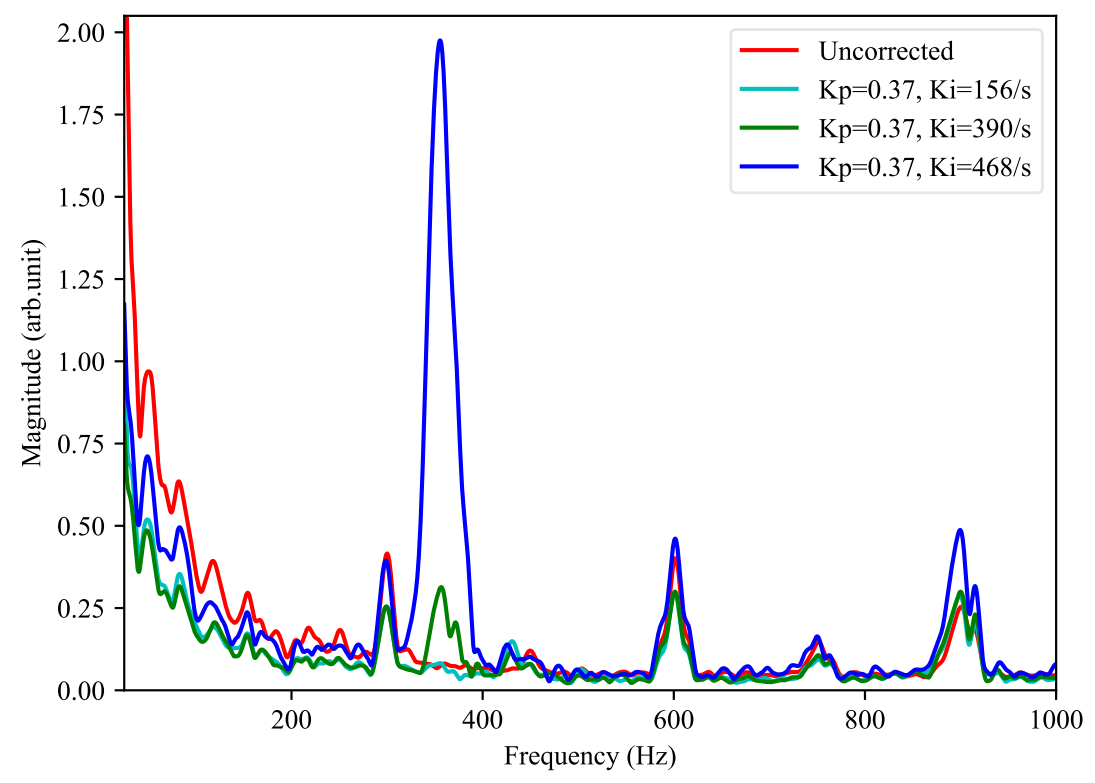

Figure 7.24 Closed orbit instability (average of the DFT of position data from all BPMs) as a function of higher controller gains for the case of using MAD-X based model ORM corresponding to the injection settings in the controller.

gains is caused by the spatial model mismatch.

- The frequency of oscillation belongs to an open-loop pole whose corresponding open-loop zero lies on the right-hand side of the complex plane but is not measured on that side of the complex plane during the open-loop full-system identification and required further measurements performed in the closed-loop configuration.

\subsubsection{Closed-loop system identification}

SIS18 COFB system was further characterized in the closed-loop configuration with the help of disturbance applied as a step change in the controller output at various times over the ramp. Figure. 7.25 shows the location of the disturbance on the schematic diagram of the feedback loop (in Laplace domain) where the transfer function from this disturbance location to output is called the load-disturbance sensitivity function [46] and can be written as,

$$
\frac{\mathbf{G}(s)}{1+\mathbf{K}(s) \mathbf{G}(s)}
$$

Instead of applying external signal as the step input additively, which would have changed the input to the system $\mathbf{G}(s)$, the beam rigidity vector inside the Libera Hadron called as c scaling in sec. 6.2.2 is changed during the ramp in order to apply a step disturbance to the feedback system. This arrangement also has effectively the same action of changing the input to the system, as a step. 


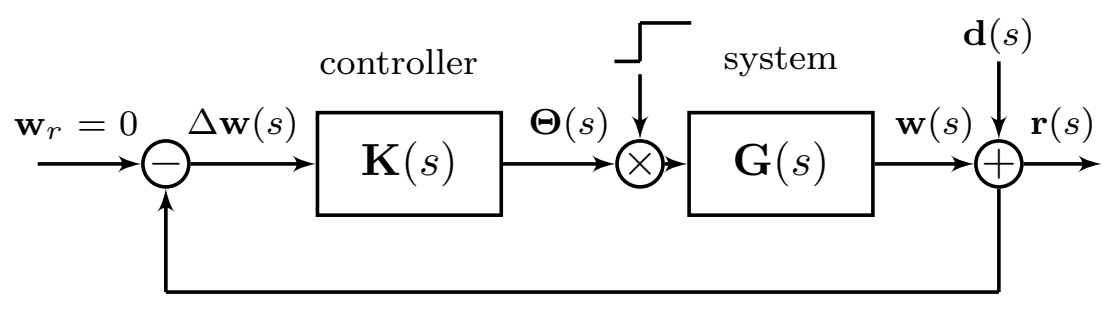

Figure 7.25 Schematic diagram of closed-loop system identification. The step input is applied at the output of the controller as a multiplication factor.

First of all, the step disturbance is applied during the ramp of dipole field gradient of $1.4 \mathrm{~T} / \mathrm{s}$, (beam parameters are already given in previous subsection). MAD-X injection ORM is used for the orbit correction so that $\rho(\mathbf{M})$ followed the blue curve (corresponding to higher values) in Fig. 7.14. The controller output is changed at times and by the factors given in table 7.5 while no beam loss occurred during the closed orbit manipulation, in this measurement. In order to compare the closed-loop response at different times of the ramp, it is necessary to chose a beam motion which has least variation during the ramp. For this purpose, the BPM position is transformed into the SVD-mode space (for details see sec. 2.7.4) as,

$$
X^{m}=\frac{1}{12} \mathbf{U}^{T} X
$$

where $\mathbf{U}$ is the SVD matrix, $X$ and $X^{m}$ are the closed orbit vectors in real and in mode spaces, respectively. Figure 7.26 shows the beam position coupled to the $4^{\text {th }}$ SVD mode as a result of step disturbances applied at different times over the ramp during the orbit correction (given in Table 7.5). The closed-loop response to a step disturbance of factor 4.2 at $210 \mathrm{~ms}$ of another slower ramp of dipole field gradient of $1.0 \mathrm{~T} / \mathrm{s}$ is also plotted in black,

Table 7.5 Information of the step disturbance applied to the SIS18 COFB system in the closed-loop configuration. The last value corresponds to the ramp of orbit correction with measured injection ORM while others are for MAD-X ORM.

\begin{tabular}{lll}
\hline \hline $\begin{array}{l}\text { Time } \\
\text { on ramp }\end{array}$ & $\begin{array}{l}\text { step change } \\
\text { (factor) }\end{array}$ & $\rho(\mathbf{M})$ \\
\hline \hline $90 \mathrm{~ms}$ & 1.9 & 1.02 \\
\hline $100 \mathrm{~ms}$ & 2.1 & 0.83 \\
\hline $110 \mathrm{~ms}$ & 2.3 & 0.82 \\
\hline $130 \mathrm{~ms}$ & 2.6 & 0.58 \\
\hline $150 \mathrm{~ms}$ & 2.9 & 0.60 \\
\hline $180 \mathrm{~ms}$ & 3.3 & 0.51 \\
\hline $200 \mathrm{~ms}$ & 3.7 & 0.58 \\
\hline \hline $210 \mathrm{~ms}$ & 4.2 & 0.19 \\
\hline
\end{tabular}




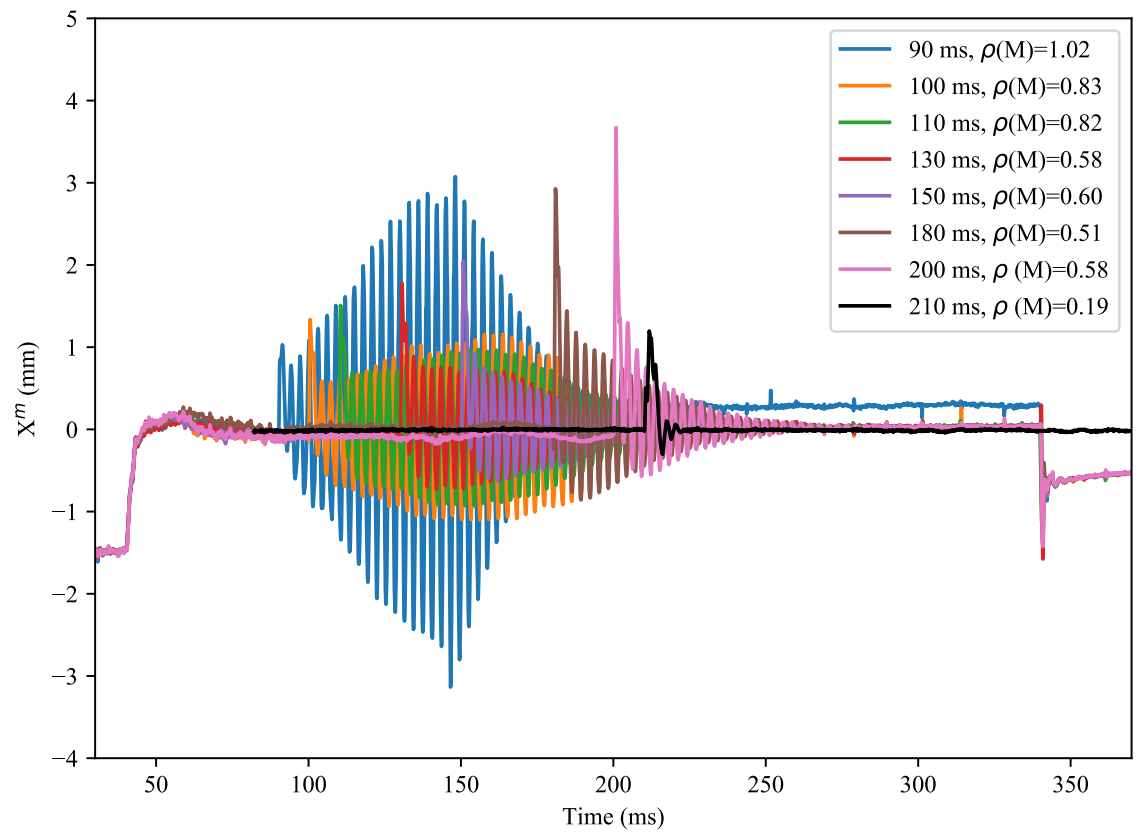

Figure 7.26 The closed-loop response of the SIS18 COFB system coupled to the one of the least varying SVD modes $\left(4^{t h}\right)$ of MAD-X ORM over the ramp, to the step inputs at various positions on the ramp (i.e. at different magnitudes of spatial model mismatch).

for comparison where $\rho(\mathbf{M})=0.19$. Qualitatively, one can see that the closed orbit oscillates as a response to the step disturbance analogous to an higher (at least $2^{\text {nd }}$ ) order system. For the step changes at $90 \mathrm{~ms}, 100 \mathrm{~ms}$ and $110 \mathrm{~ms}$, closed orbit oscillations can be seen growing in the beginning and later decaying meaning that the COFB system passed through instability region over the ramp at these times in response to the step disturbance. On the other hand, the oscillations only decay for the rest of the times even for larger amplitudes of step. It is important to mention that the controller parameters are identical for all ramps of Fig. 7.26 ( $K_{p}=0.12$ and $K_{i}=469 / \mathrm{s}$ ) except for step response at $210 \mathrm{~ms}$ for which $K_{p}=0.19$ and $K_{i}=2343 / s$. These values are chosen to have a stable closed-loop response in the absence of step disturbance. The closed orbit oscillations die very fast for the case of $210 \mathrm{~ms}$ because of smaller $\rho(\mathbf{M})=0.19$ even for a higher amplitude of step disturbance.

For the quantitative analysis, beam oscillations (in mode space) are curve fitted one by one, with the following sinusoidal function,

$$
X^{m}(t)=A e^{\lambda t} \sin (\omega t+\phi)
$$

where $A$ is the amplitude, $\lambda$ is the decay constant, $\omega$ is the frequency of oscillations and $\phi$ is the phase, respectively. The fitting is done over a time window of 14 ms moved by $100 \mu$ s so that the change in the parameters of Eq. 7.12 could be measured as a function of time on the ramp and consequently as a function of $\rho(\mathbf{M})$. 


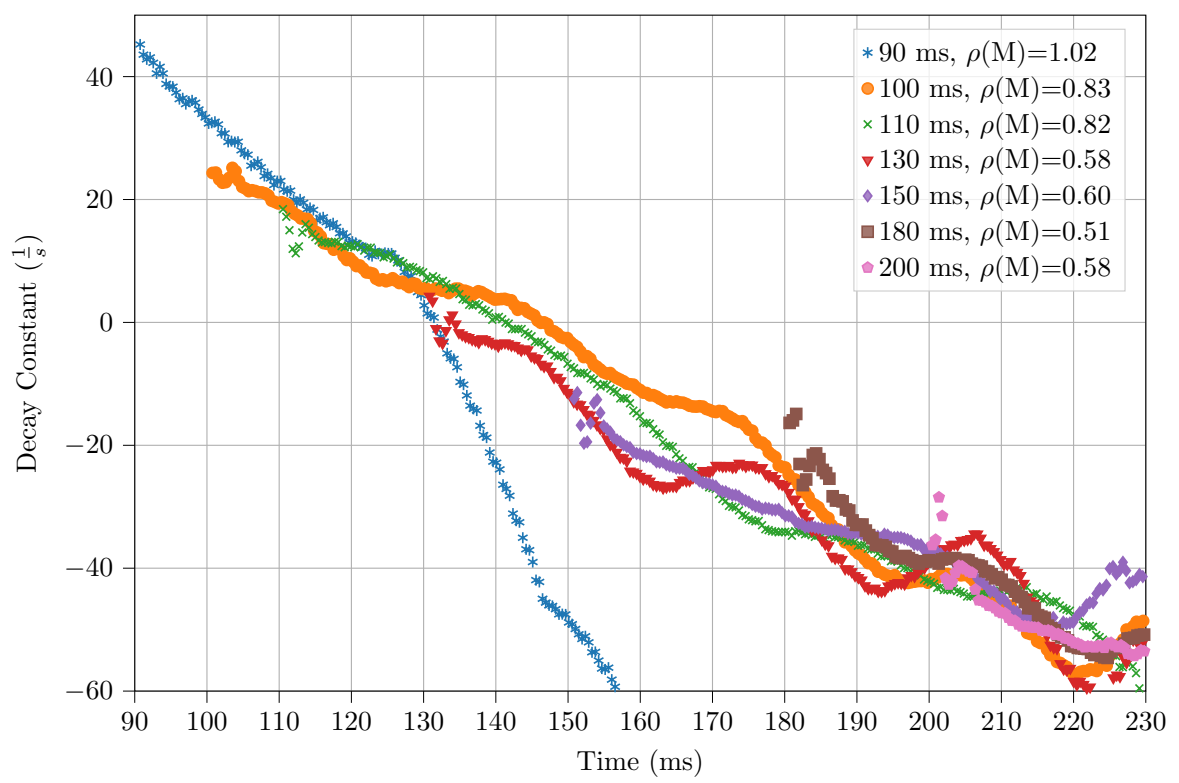

Figure 7.27 Decay constant of the beam motion coupled to one of the least varying SVD modes $\left(4^{\text {th }}\right)$ of MAD-X ORM over the ramp, as a response to the step change at various times on the ramp (i.e. at different magnitudes of spatial model mismatch), plotted in Fig. 7.28

Eq. 7.12 is the well-known solution of the second order system (e.g. harmonic oscillator) where the characteristic frequency $\omega$ is the imaginary part of the dominant pole of the transfer function given as,

$$
H(s)=\frac{A \omega^{2}}{s^{2}+\lambda s+\omega^{2}}
$$

where the pole is defined as $p=\lambda \pm j \omega \sqrt{1-\left(\frac{\lambda}{\omega}\right)^{2}}$ and the sign of $\lambda$ infers the location of the pole on the right- (positive $\lambda$ ) or the left- (negative $\lambda$ ) hand side of the complex plane (for details see sec. 2.8.1).

The decay constants $\lambda_{\mathrm{s}}$ extracted from curve fitting, for each step response of Fig. 7.26 are plotted as function of time of the ramp in Fig. 7.27. An instability of the COFB system is clearly evident from the positive sign of $\lambda$ up to $130-140 \mathrm{~ms}$ for the response to step disturbance at $90 \mathrm{~ms}, 100 \mathrm{~ms}$ and $110 \mathrm{~ms}$. While, a continuous decrease in the decay constant over the ramp resulting into the sign change from positive to negative shows a transition from instability to stability of the COFB system, for the above mentioned times of step disturbances. For the rest of the times, the decay constants remain negative even for higher step change in the controller output at $130 \mathrm{~ms}$ to $200 \mathrm{~ms}$. The comparison of $\rho(\mathbf{M})$ (blue curve of Fig. 7.14 and the decay constants of Fig. 7.27 confirm the direct relation between the instability of the COFB system and the larger $\rho(\mathbf{M})$.

Figure 7.28 shows the movement of the poles (extracted by the fitting of step responses of Fig. 7.27) on the complex plane, for all step changes over the ramp, where the frequency 


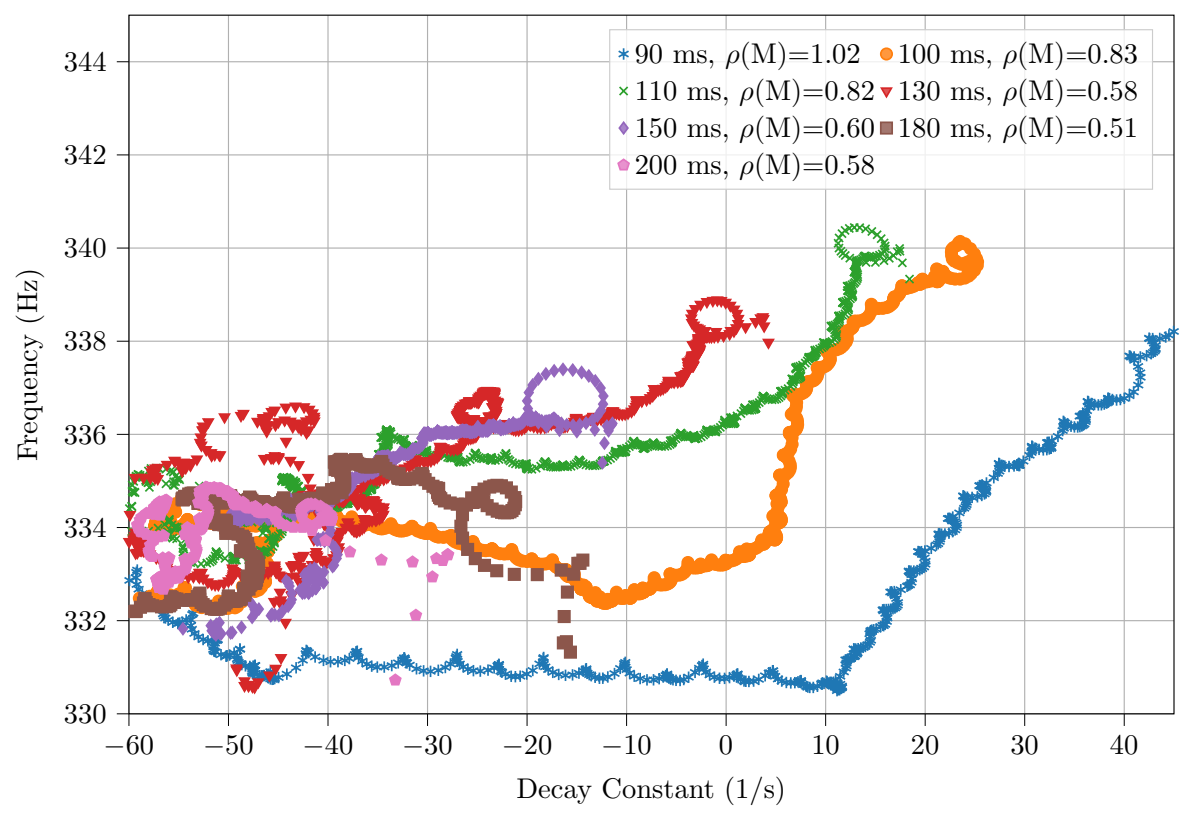

Figure 7.28 Pole movement as a function of model mismatch over the ramp measured by the fitting of step responses of beam motion coupled to the one of the least varying SVD modes $\left(4^{\text {th }}\right)$ of MAD-X ORM over the ramp, plotted in Fig. 7.26

of the oscillations is plotted versus the decay constant. One can see that the frequencies of oscillations vary only within a small window of 330 and $340 \mathrm{~Hz}$ and is centered around 335 $\mathrm{Hz}$. It is the same frequency as reported in the previous subsection for the instability caused by the higher controller gains in the region of higher $\rho(\mathbf{M})$.

As mentioned before, the measured closed-loop response to the step disturbance can be modelled with the help of transfer function given in Eq. 7.10 and is also simulated for the effective controller gains corresponding to $\rho(\mathbf{M})=0.58$ i.e. $K(s) /(1-0.58)$ (for details see sec. 5.7) and is compared with the measured response to the step disturbance at $200 \mathrm{~ms}$. The open-loop transfer function is optimized as a result of simulation in order to overlap the measured and simulated responses as shown in Fig. 7.29. The fitted open-loop transfer function is given in the zero-pole-gain form as,

$$
\text { sys }=e^{-0.00033 s} \frac{7702.7\left(s+1.071 \times 10^{4}\right)\left(s^{2}-494 s+4.735 \times 10^{6}\right)}{\left(s^{2}+185.6 s+5.044 \times 10^{6}\right)\left(s^{2}+9033 s+1.021 \times 10^{8}\right)}
$$

which has a conjugate pole pair at $-14.8 \pm 357 j$ and conjugate zero-pair at $39.3 \pm 344 j$. The frequencies are slightly different from those measured during open-loop system identification which is performed for each of the corrector channel, separately while the transfer function of Eq. 7.14 is extracted from the response of the closed orbit including all 12 correctors along with their slew-rate limitation. The closed-loop system identification has confirmed that the zero corresponding to the pole of frequency of closed orbit oscillations lies on the right-hand side of the complex plane, although is not measured during the open-loop system identification on that side of the complex plane. 


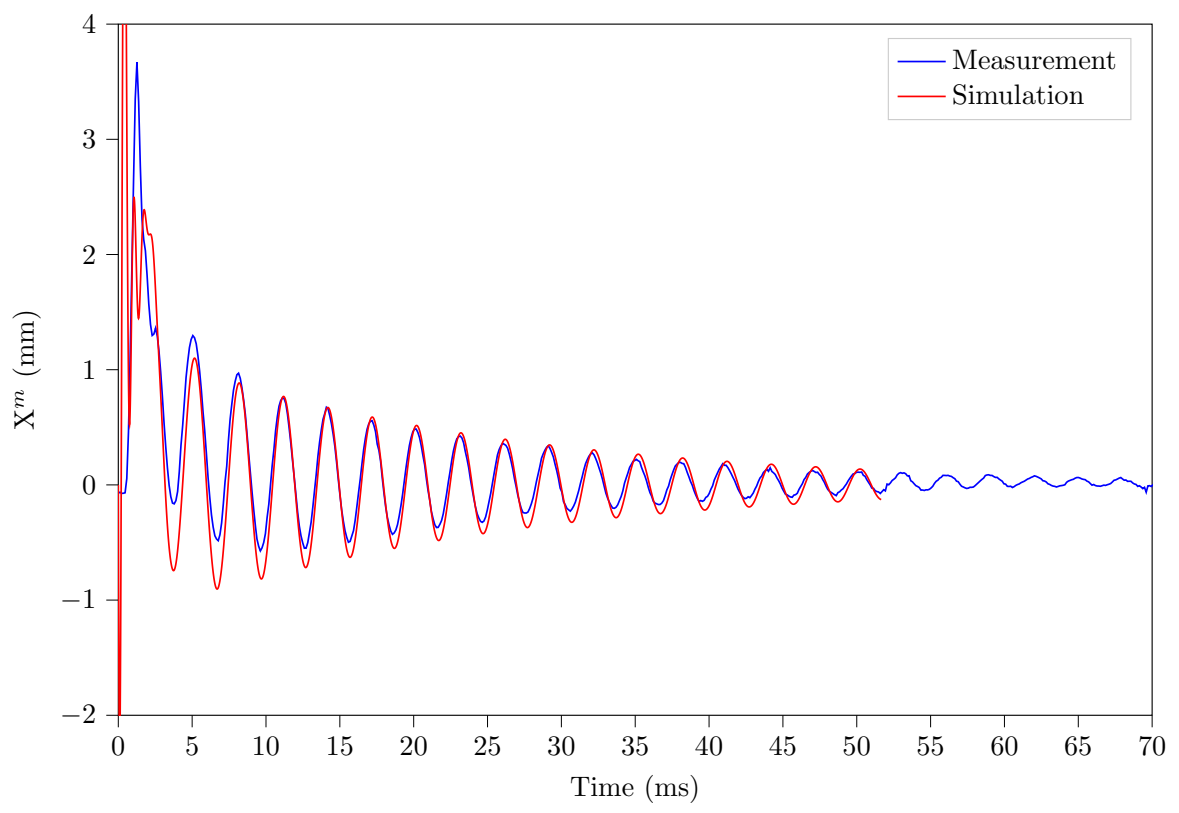

Figure 7.29 Closed-loop response to the step disturbance at $200 \mathrm{~ms}$ of the ramp for which $\rho(\mathbf{M})=0.58$. Further details in text below.

\subsection{Summary}

In this chapter, the commissioning and the operation of a fast closed orbit feedback system for the on-ramp orbit correction in the horizontal plane of SIS18 synchrotron has been described. The important steps and the results can be summarized as follows:

- The commissioning of the fast COFB system is realized using Libera Hadron (hardware details in chapter 6) for BPM data processing and controller output calculation. 12 corrector magnets and 12 BPMs of SIS18 are engaged by the COFB system.

- The sub-system identification including corrector magnets, their power converters and adaptive control units is performed as a first step, using sinusoidal (amplitude $0.7 \mathrm{~A}$ ) and pulse signals (amplitude 0.7, 6, 12, 18 and $36 \mathrm{~A} / 10 \mu \mathrm{s}$ ) as excitation in order to measure the transfer functions. It is found that the slew-rate limitation implemented in the power converters of the corrector magnets changes the effective transfer function for higher input slew-rates. The salient effects of slew-rate limitation are the movement of zeros corresponding to the poles of lower frequencies to the right-hand side of the complex plane along with the reduction of effective bandwidth of the system. Moreover, it is found that 2 out of 12 corrector channels have different transfer functions, during these measurements.

- The full-loop system identification is also performed using sinusoidal excitation (amplitude $0.78 \mathrm{~A}$ ) and new pole-zero pairs in the vicinity of $300-400 \mathrm{~Hz}$ are measured for 9 out of 10 similar corrector channels of subsystem identification. 
- The transfer function measured as result of full-loop system identification is used for the estimation of the controller gains. The estimation is only an initial guess as the effective transfer function is expected to change during the ramp due to variable slew-rate of power converters.

- The orbit response matrix (ORM) is measured over the entire ramps for two different machine settings using a sinusoidal excitation of amplitude corresponding to $1 \mathrm{mrad}$ corrector strength, one by one for each corrector magnet. The spatial model mismatch is calculated (in terms of $\rho(\mathbf{M})$ ) over the ramp with respect to MAD-X model as well measured ORM of injection settings. The maximum $\rho(\mathbf{M})$ at extraction energy relative to measured injection ORM is comparable to the theoretical value simulated in Chapter 5. It is also found that the MAD-X model has an intrinsic large mismatch $(\rho(\mathbf{M})=0.4-0.6)$ with respect to the measured ORM even for injection settings. The exact reason for such a mismatch is not known and further investigation is carried out to correct the MAD-X model.

- The closed orbit correction is demonstrated with the help of MAD-X and the measured ORM corresponding to injection settings for fixed controller gains for each ramp. The maximum achieved bandwidth of orbit correction is $600 \mathrm{~Hz}$, which reduced to $300 \mathrm{~Hz}$ in case of larger spatial model mismatch i.e. for using MAD-X ORM in the controller.

- The effect of spatial model mismatch on the stability of the COFB system is demonstrated experimentally and it is shown that the spectral radius $\rho(\mathbf{M})$ can alone define the performance and stability of a COFB system, even in fast regime, in agreement with the theoretical conclusions made in Chapter 5 as a result of simulations.

- The frequency of oscillations measured in case of COFB system instability due to the larger $\rho(\mathbf{M})$ is around $335 \mathrm{~Hz}$. Although a conjugate-pair of poles frequency $322 \mathrm{~Hz}$ has been measured during the open-loop full-system identification, their corresponding zeros are measured on the left-hand side of the complex plane, predicting that closed loop system should not get unstable on this frequency. Either, the zeros lie on the right-hand side of the complex plane in reality and are not measured or they appear on that side due to the high slew-rates of the corrector-strength variation during the ramp.

- In order to further confirm the dominant poles of system, a closed loop system identification is performed with the help of step change in the controller output, and the system response is made to oscillate (measured as closed orbit oscillations). The measurement also confirm the dominant pole movement from left- to the right-hand side of the complex plane as a result of larger $\rho(\mathbf{M})$ is centered around $335 \mathrm{~Hz}$.

- The closed orbit instability due to spatial model mismatch presented in this chapter for the fast regime is the first demonstration of its kind in the field of orbit correction. But a quantitative analysis can be further extended for the explanation of the conclusions emerged during experiments having an improved open-loop system identification and simulations. 


\section{Chapter Eight}

\section{Summary and outlook}

This chapter summarizes the thesis for its contributions made in the field of closed orbit correction in pursuit of the realization of fast and robust closed orbit feedback system for SIS18 synchrotron of FAIR project.

\subsection{Summary}

Closed orbit feedback (COFB) system is implemented in most synchrotrons for the correction of transverse distortions in the closed orbit, caused by the time-dependent and fixed dipolar field errors. The transverse beam position is measured with the help of beam position monitors (BPMs), and orbit correction is performed by correctors, which are dedicated dipole magnets. The required bandwidth of disturbance rejection and the allowed residual depends upon the type of synchrotron. For example, in the storage rings of light sources the bandwidth of interest lies from DC to $150 \mathrm{~Hz}$ while the position deviation is required to be less than several $\mu \mathrm{m}$. This is in agreement with the allowed position deviation of less than $10 \%$ of the beam size which is a generally accepted criterion in the light source community. SIS18 of the FAIR project is a ramping hadron synchrotron and will serve as the booster ring for the upcoming SIS100 synchrotron. Previously, the orbit correction in SIS18 has been performed only at injection and extraction where the optics is fixed and only against DC offsets. While high frequency perturbations up to $1 \mathrm{kHz}$ exist throughout the ramp, requiring a fast on-ramp COFB system, which has been realized as the final goal of this thesis. It is also worthwhile to mention that a cycle-to-cycle feedback system for SIS18 is also under development for the past few years.

SIS18 has some peculiar behaviours as compared to the storage rings of the light sources as well as other ramping hadron synchrotrons (e.g. Large Hadron Collider), the most important of them being the on-ramp optics variation, very short lengths of the ramps (200 ms to $1 \mathrm{~s}$ ) and the cycle-to-cycle variation of beam parameters. To the best knowledge of the author, this is the first realization of a fast on-ramp COFB system which is also robust against above mentioned features of SIS18.

In Chapter 4 , first of all, the existing methods for global orbit correction are reviewed in order to deal with fast on-ramp optics variation. The most commonly used method relies on the singular value decomposition (SVD) of the orbit response matrix (ORM) due to its robustness regarding the (pseudo-)inversion of the ORM for the corrector-strengths calculation.

Certain limitations of the SVD-based method are pointed out, in the context of on-ramp 
orbit correction. SVD is a technique for decomposing any matrix (ORM, in case of orbit correction) into two left and right orthogonal matrices and one middle diagonal matrix, whose diagonal entries are called singular values. The columns of orthogonal matrices are regarded as SVD modes that are uniquely coupled through singular values. In this way, the closed orbit is effectively resolved into the SVD mode space, analogous to the vector resolution into its Cartesian components. But the SVD modes have no clear analytical interpretation, and consequently, the closed orbit is resolved into a mode space that has no physical meaning in order to relate different beam phenomena and their variation over the ramp with the resolved components of the closed orbit. Similarly, there is no apparent analytic way of associating uncertainties in the lattice parameters, e.g. tune to the singular values. The later limitation was also reported previously in the literature. The most critical limitation is regarding the update of the ORM during the ramp. There is an interconnection between all three SVD matrices, and the update of the (pseudo-)inverse of ORM in the feedback controller requires the calculation and updating of all three matrices for each time step of the ramp.

A new method for the decomposition and the (pseudo-)inversion of the ORM is presented in this thesis, relying on the symmetry exploitation in the ORM. A special kind of symmetry called circulant or block-circulant (depending upon the number of BPMs and correctors per cell) arises in the ORM if the synchrotron has a symmetry in its cells, which is the case for most of the synchrotrons. For the broken symmetry e.g. due to the placement of insertion devices in light sources, a nearest-circulant approximation has also been worked out, making the method applicable to a vast majority of synchrotrons. The method has the following powerful implications: Firstly, it simplifies the ORM decomposition by the discrete Fourier transform (DFT) of its only single row or a single block of rows that repeat cyclically throughout the ORM to make it circulant or block-circulant, respectively. This means a significant reduction in the computational complexity and the required memory resources in comparison to SVD. The complex Fourier coefficients form the middle diagonal matrix while left and right orthogonal matrices are standard Fourier matrices, which are constant for any circulant ORM of the same size. Secondly, in this case, the mode space comprises of analytical sine or cosine functions that give solutions to most of the limitations of the SVD-based method discussed above. Since the Fourier matrices are fixed even if the ORM changes (retaining the symmetry), one needs to update only the Fourier coefficients over the ramp for a changing machine optics, instead of updating all three matrices at each time step of the ramp. An equivalence of the SVD- and DFT-based decomposition is presented for the circulant ORMs, which provides a physical interpretation also to the SVD modes, in this case.

The circulant symmetry is explored in the vertical ORM of SIS18 synchrotron, while application of nearest-circulant and nearest-block circulant approximation is demonstrated for the horizontal ORM of SIS18 and both ORMs of SIS100 synchrotrons. The advantages of symmetry exploitation are also discussed, including computational complexity, and demonstrated with the help of simulations for the orbit correction in case of "missing" BPMs (e.g. ignoring a possibly faulty BPM position reading). Moreover, the better manipulation of a beam phenomenon i.e. the dispersion-induced orbit shift is demonstrated with the help of Fourier-mode space over SVD-mode space, experimentally in the horizontal plane of SIS18 using the nearest-circulant approximation. 
Another significant contribution of this thesis discussed in Chapter 5 is the performance and stability analysis of COFB systems against the ORM mismatch between the synchrotron and the feedback controller. This corresponds to the on-ramp orbit correction scenario using a fixed (pseudo-)inverse of the ORM or updating it after larger time intervals (due to technical limitations), while the ORM in the synchrotron changes continuously during the ramp. Interestingly, no study was found in the literature to predict a COFB system's behaviour for the comparable magnitudes of ORM mismatch observed for SIS18. The uncertainty modeling in the ORM for small tune variations was found in literature based upon the small gain theorem of the control theory, which yields very stringent stability margins and hence proved to be impractical for the analysis of the SIS18 COFB system.

COFB system is analyzed by splitting the system's model into two separate parts, i.e. temporal part containing the time response of all hardware in the feedback loop and the spatial part representing the ORM, in this thesis. Feedback systems are divided into two regimes, i.e. slow and fast regimes. In the slow regime, the time step of orbit correction is at least an order of magnitude longer than system delays and open-loop time constants. The motivation for this classification is first to ignore the temporal parts of the system and the controller in the slow regime to define stability criteria only in terms of the ORMs and then modify the results for the case of including temporal parts for the fast regime. The stability criteria defined for the slow regime called spectral radius condition (Chapter 5) is found to give a margin higher than the small gain theorem by a factor equal to the condition number of the controller ORM. It is emphasized that the spectral radius condition can be applied to any kind of ORM variation as it only depends upon the relative discord between the two ORMs hence presenting it as a practical figure of merit for day-to-day COFB system operation for every facility. A relation of the spectral radius to a measurable quantity, i.e. the correction ratio defined as the ratio of residual orbit (RMS value) to the perturbed orbit, after a single correction is also derived.

Various sources of spatial model mismatch in SIS18, including on-ramp ORM variation, intensity-dependent tune shift and beta beating, are considered in simulations and the spectral radius is calculated for ORM variation with respect to injection settings, zero tune shift and zero beta beating, respectively. More ORM variation is found in the horizontal plane than in the vertical plane, which is consistent with the fact that lattice settings variation occurs primarily in the horizontal plane. These simulations predicted that SIS18 COFB system should not be unstable even for the maximum on-ramp model mismatch for the slow regime of operation in both planes, for the calculated magnitudes of ORM mismatch. For the intensity-dependent tune shift, it is found that the instability of the COFB system depends not only upon the magnitude of the tune shift but also on the direction of tune shift towards or away from integer value. Tune shift away from integer value ensures the COFB system stability even for a larger magnitude of tune shifts. The validity of the spectral radius condition is verified with simulations for SIS18 and experimentally at COSY Jülich by observing the correction ratio over many iterations for various sources and magnitudes of the ORM mismatch. It is further found that the beta beating itself is not a crucial source of feedback instability unless accompanied by a tune shift towards an integer value.

The stability criteria framework is extended to the fast feedback regime taking system and controller dynamics into account, and the transfer functions are derived in terms of the 
spectral radius. It is found quantitatively and demonstrated with the help of simulations that the achievable bandwidth of disturbance rejection decreases with the increase of ORM mismatch. As a result, to reach a certain bandwidth throughout the ramp, the ORM update rate has to be changed. Moreover, the required scaling of the controller parameters with ORM mismatch is also derived in order to avoid closed-loop instability.

Along with the investigations mentioned above, SIS18 COFB system has also been commissioned for the horizontal plane. Chapter 6 presents the details of the main hardware resource which is a Slovenian in-kind contribution to FAIR. It is a dedicated device named Libera Hadron used for the BPM data processing, controller implementation and the correctorstrength calculation. The pure delay measurement and fixed-point arithmetic tests are performed for Libera Hadrons, and three of them are integrated with 12 BPMs and 12 corrector magnets to realize the global COFB system.

Chapter 7 contains the further details of commissioning of the SIS18 COFB system along with the final results of on-ramp orbit correction. The sub-system identification is performed for each corrector magnet along with its power converter and the adaptive control unit with the help of sinusoidal input as well as pulse inputs of various amplitudes, to estimate the transfer functions. The power converters have a slew-rate limitation which is also recorded during the sub-system identification. A beam-based full-loop system identification is also performed for each corrector magnet using the sinusoidal excitation. The controller parameters for the Proportional Integral (PI) controller implemented in Libera Hadron are estimated based upon full-system identification. The estimation is taken as an initial guess since the effective transfer function is expected to change during the ramp for higher slew-rate of corrector strengths, as predicted by sub-system identification. ORM measurements are also performed over the entire ramps for two different machine settings. Hence, the spectral radius is measured with respect to the MADX model and the measured injection ORMs. Unexpectedly, the MADX model has shown an intrinsic mismatch with respect to the measured ORM, even at injection settings.

On-ramp orbit correction is demonstrated for the fixed controller parameters and for the fixed injection ORM for corrector-strength calculation. The maximum achieved bandwidth of orbit correction is $600 \mathrm{~Hz}$ if the measured injection ORM is used, while for using the MADX model, the orbit correction up to $300 \mathrm{~Hz}$ has been be achieved.

The experiments are further extended including closed-loop system identification, to verify the theoretical conclusions regarding the stability and performance of the COFB system against ORM mismatch in the fast regime.

\subsection{Outlook}

- One reason for the deviation from circulant or block-circulant symmetry in the ORMs of the majority of storage rings is the placement of insertion devices in $3^{\text {rd }}$ generation light sources. Modern facilities are regaining the symmetries in their ORMs, e.g. Diamond-II of the UK, ALBA of Spain, Sirius of Brazilian Light Source and NSLS-II of Brookhaven National Laboratory. Symmetry exploitation and the DFT-based decomposition of the ORM can be applied as an alternate to the SVD for modern synchrotrons and light 
sources. Even a recent effort has already been reported to utilize the computational benefits of symmetry exploitation for the Diamond-II COFB system [96].

- The spectral radius condition that has been shown to yield practical margins of ORM mismatch between the controller and the synchrotron, for the stability of the COFB system can be utilized by any synchrotron facility regardless of the nature of the ORM mismatch. It can be used as a figure of merit for the performance and stability of a COFB system for day-to-day operation in case of ORM variations. Typically, efforts are made on the hardware side of the COFB system in order to increase the bandwidth for disturbance rejection. Based upon the conclusions drawn in this thesis, it is strongly recommended that the spatial model mismatch should also be investigated thoroughly even for the fixed-lattice storage rings as it can have a detrimental effect on the achievable bandwidth.

- SIS18 COFB system has been tested using the SVD-decomposed fixed ORM throughout the ramp in the measurements presented in this thesis. In order to benefit from the symmetry exploitation in terms of using only DFT-coefficients for updating the ORMs, the FPGA design of the Libera Hadron needs to be upgraded for storing the complex Fourier coefficients and matrices for the corrector-strength calculation.

- Experimental results presented in this thesis are the first-ever demonstrations of the effect of spatial model mismatch on the stability and performance of the COFB systems in slow and fast regimes. However, an improvement in the open-loop full-system identification, including the effect of slew-rate, is required for the quantitative understanding of the closed-loop behaviour in the presence of spatial model mismatch and for reproducing it in simulations.

- For the vertical plane of SIS18, the installation of adaptive control units of power supplies and the addition of one more Libera Hadron is required for the commissioning of the COFB system in this plane. The system identification and the ORM measurement over the ramp will also be required in the vertical plane. 


\section{REFERENCES}

[1] Guenther Rehm. "Achieving and Measuring Sub-micrometer Beam Stability at $3^{\text {rd }}$ Generation Light Sources". In: Journel of Physics: Conference Series 425 (2013).

[2] D. Bulfone. "Overview of Fast Beam Position Feedback Systems". In: Conf. Proc. C 0806233 (2008). Ed. by Ivan Andrian and Christine Petit-Jean-Genaz, TUZM02.

[3] Sandira Gayadeen. "Synchrotron Electron Beam Control". Thesis, University of Oxford, 2014.

[4] Weixing Cheng et al. "Characterization of NSLS2 Storage Ring Beam Orbit Stability". In: $4^{\text {th }}$ International Beam Instrumentation Conference. 2016, THALA02. DOI: 10. 18429/JACoW-IBIC2015-THALA02.

[5] E. Plouviez et al. "The new fast orbit correction system of the ESRF storage ring". In: $10^{\text {th }}$ European Workshop on Beam Diagnostics and Instrumentation for Particle Accelerators (DIPAC 2011), Hamburg, Germany. 2011.

[6] G. Kube et al. "Overview of the Diagnostics Systems of PETRA III". In: Conf. Proc. C 0806233 (2008). Ed. by Ivan Andrian and Christine Petit-Jean-Genaz, TUPC111.

[7] R. J. Apsimon et al. "Design and operation of a prototype interaction point beam collision feedback system for the International Linear Collider". In: Phys. Rev. Accel. Beams 21 (12 Dec. 2018), p. 122802. DOI: 10.1103/PhysRevAccelBeams.21.122802. URL: https://link.aps.org/doi/10.1103/PhysRevAccelBeams.21.122802.

[8] L H Yu. "The Performance of a Fast Closed Orbit Feedback System with Combined Fast and Slow Correctors". In: $11^{\text {th }}$ European Particle Accelerator Conference (EPAC 2008); Genoa, Italy (2008). URL: https://www.osti.gov/biblio/939963.

[9] J. Wenninger. "LHC orbit feedback - architecture and operational experience". In: Joint ARIES Workshop on Electron and Hadron Synchrotrons (2018). URL: https: //indico.cern.ch/event/743699/contributions/3072646/.

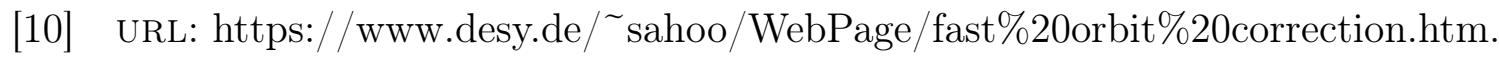

[11] B. Autin and Y. Marti. "Closed orbit correction of alternating gradient machines using a small number of magnets". In: European Organization for Nuclear Research Report No. CERN-ISR-MA/73-17 (1973).

[12] L.H. Yu et al. "Real time harmonic closed orbit correction". In: Nuclear Instruments and Methods in Physics Research Section A: Accelerators, Spectrometers, Detectors and Associated Equipment 284.2 (1989), pp. 268-285. ISSN: 0168-9002. DOI: https: 
//doi.org/10.1016/0168-9002(89)90292-1. URL: https://www.sciencedirect.com/ science/article/pii/0168900289902921.

[13] Y. Cheng. "Dynamic closed orbit correction". In: Proceedings of the Particle Accelerator Conference (PAC 1993), Washington, D.C. (1993), p. 2269.

[14] Y. Chung, G. Decker, and K. Evans. "Closed orbit correction using singular value decomposition of the response matrix". In: Proceedings of International Conference on Particle Accelerators. 1993, 2263-2265 vol.3. DOI: 10.1109/PAC.1993.309289.

[15] K. Wille. The Physics of Particle Accelerators. An Introduction. Oxford University Press, 1996.

[16] O. Bruning and S. Myers. Challenges and Goals For Accelerators In The XXI Century. World Scientific, 2016.

[17] M. Sands. "The physics of electron storage rings: An introduction". In: Stanford Linear Accelerator Center Report No. SLAC-121 (1970).

[18] Helmut Wiedemann. Particle Accelerator Physics. Introduction to Accelerator Physics. Springer, 2015.

[19] Helmut Wiedemann. Particle Accelerator Physics. Circular accelerators. Springer, 2015.

[20] P. Elleaume. "Undulators and wigglers for the new generation of synchrotron sources". In: Journal de Physique IV Colloque 04 (1994), pp. C9-3-C9-10.

[21] M. Martini. "An introduction to transverse beam dynamics in accelerators". In: CERN/PS 9611 (PA) (1996).

[22] V. Ziemann. "Response matrices in strongly coupled storage rings with a radiofrequency system constraining the revolution time". In: Phys. Rev. ST Accel. Beams 18 (5 May 2015), p. 054001. DOI: 10.1103/PhysRevSTAB.18.054001. URL: https: //link.aps.org/doi/10.1103/PhysRevSTAB.18.054001.

[23] URL: https://www.lnls.cnpem.br/the-lnls/history-of-the-synchrotron-light-sources/.

[24] J. Klute et al. "The Petra III fast orbit feedback system". In: Proceedings of DIPAC2011. 2011, MOPD76.

[25] L. Torino and U. Iriso. "Transverse beam profile reconstruction using synchrotron radiation interferometry". In: Phys. Rev. Accel. Beams 19 (12 Dec. 2016), p. 122801. DOI: 10.1103/PhysRevAccelBeams.19.122801. URL: https://link.aps.org/doi/10.1103/ PhysRevAccelBeams.19.122801.

[26] Glenn Decker. "Beam stability in synchrotron light sources". In: Proceedings of DIPAC. 2005, ITWM01.

[27] J. Feikes et al. "Orbit Stability at BESSY". In: Proceedings of the 2005 Particle Accelerator Conference. 2005, pp. 2366-2368. DoI: 10.1109/PAC.2005.1591112. 
[28] Åke Andersson et al. "Diagnostics at the Max IV $3 \mathrm{GeV}$ Storage Ring During Commissioning". In: 5th International Beam Instrumentation Conference. 2017, MOAL02. DOI: $10.18429 /$ JACoW-IBIC2016-MOAL02

[29] R. O. Hettel. "Beam stability at light sources (invited)". In: Review of Scientific Instruments 73.3 (2002), pp. 1396-1401. DOI: 10.1063/1.1435812. eprint: https: //doi.org/10.1063/1.1435812, uRL: https://doi.org/10.1063/1.1435812.

[30] Alexander Wu Chao et al. Handbook of Accelerator Physics and Engineering. 2nd. WORLD SCIENTIFIC, 2013. DOI: 10.1142/8543. eprint: https://www.worldscientific. com/doi/pdf/10.1142/8543. URL: https://www.worldscientific.com/doi/abs/10.1142/ 8543 .

[31] Andy Wolski. "Storage Ring Design Part 1: Beam Dynamics with Synchrotron Radiation". In: Joint US-CERN-Japan-Russia School on Particle Accelerators (2011).

[32] URL: https://www.bnl.gov/rhic/

[33] URL: https://www.desy.de/forschung/anlagen__projekte/hera/index_ger.html.

[34] URL: https://home.cern/science/accelerators/large-hadron-collider.

[35] URL: https://j-parc.jp/c/en/

[36] R. J. Steinhagen. "LHC Beam Stability and Feedback Control - Orbit and Energy -". Presented on 22 Jun 2007. 2007. URL: http://cds.cern.ch/record/1054849.

[37] Kentaro Harada et al. "Orbit correction using an eigenvector method with constraints for synchrotron radiation sources". In: Nuclear Instruments and Methods in Physics Research Section A: Accelerators, Spectrometers, Detectors and Associated Equipment 604.3 (2009), pp. 481-488. ISSN: 0168-9002. DOI: https://doi.org/10.1016/j.nima.2009. 03.238. URL: https://www.sciencedirect.com/science/article/pii/S0168900209006895.

[38] Aharon Friedman and Eva Bozoki. "Use of eigenvectors in understanding and correcting storage ring orbits". In: Nuclear Instruments and Methods in Physics Research Section A: Accelerators, Spectrometers, Detectors and Associated Equipment 344.2 (1994), pp. 269-277. ISSN: 0168-9002. DOI: https://doi.org/10.1016/0168-9002(94)90075-2. URL: https://www.sciencedirect.com/science/article/pii/0168900294900752.

[39] Angelina Parfenova. "Linear and nonlinear Response Matrix and its application to the SIS18 synchrotron". https://www.gsi.de/documents/DOC-2008-Jul-31.html;Universität Frankfurt/M., Diss., 2008. PhD thesis. Universität Frankfurt/M., 2008, 150 p. URL: https://repository.gsi.de/record/55269.

[40] J. P. Koutchouk. "Trajectory and closed orbit correction". In: $3^{\text {rd }}$ Joint US-CERN School on Particle Accelerators: Frontiers of Particle Beams, Observation, Diagnosis and Correction. Jan. 1989, pp. 0046-64.

[41] W.H. Press et al. Numerical Recipes: The Art of Scientific Computing. 3rd ed. Cambridge University Press, 2007. ISBN: 9780521880688.

[42] G. H. Golub and C. F. V. Loan. Matrix Computations, Chapter 2. The Johns Hopkins University Press, 2013. 
[43] V. Ziemann. "Corrector ironing in the SLC final focus". In: Proceedings of the Particle Accelerator Conference (PAC 1993), Washington, D.C., 1993 (1993), p. 498.

[44] Leo Depuydt and Marshall Clagett. "Ancient Egyptian Science, Vol. II: Calendars, Clocks, and Astronomy". In: Journal of the American Oriental Society 118.1 (1998), p. 75. DOI: $10.2307 / 606303$.

[45] R.N. Webb. James Watt: Inventor of a Steam Engine. Immortals of mankind. Franklin Watts Limited, 1972. ISBN: 9780851662190.

[46] Karl Johan Astrom and Richard M. Murray. Feedback Systems: An Introduction for Scientists and Engineers. Princeton University Press, 2008. ISBN: 0691135762.

[47] S. Skogestad and I. Postlethwaite. Multivariable Feedback Control: Analysis and Design. Wiley, 2005. ISBN: 9780470011683.

[48] G.F. Franklin, J.D. Powell, and M.L. Workman. Digital Control of Dynamic Systems. Addison-Wesley series in electrical and computer engineering: Control engineering. Addison-Wesley Publishing Company, 1990. ISBN: 9780201119381.

[49] S. H. Kim. "Calculation of pulsed kicker magnetic field attenuation inside beam chambers". In: Technical Report LS-291, Advanced Proton Source (2001).

[50] URL: https://en.wikiversity.org/wiki/Table_of_physical_constants.

[51] SIS-SV 067.011.070s, technical drawings for SIS18 vacuum chambers.

[52] Peter Forck, P. Kowina, and Dmitry Liakin. "Beam Position Monitors". In: Synchrotron Radiation News 1 (Jan. 2008).

[53] Rahul Singh. "Tune Measurement at GSI SIS-18: Methods and Applications". Technische Universität, Darmstadt, 2014.

[54] Forck Peter. "Lecture Notes on Beam Instrumentation and Diagnostics". In: Joint University Accelerator School (2020).

[55] A. Reiter and R. Singh. "Comparison of beam position calculation methods for application in digital acquisition systems". In: Nuclear Instruments and Methods in Physics Research Section A: Accelerators, Spectrometers, Detectors and Associated Equipment 890 (2018), pp. 18-27. ISSN: 0168-9002. DOI: https://doi.org/10.1016/ j. nima.2018.02.046. URL: http:// www . sciencedirect.com/science/article/pii/ S0168900218302006.

[56] Rahul Singh et al. "Digital Processing of Pick-up Signals for Position and Tune Determination". In: 4th International Beam Instrumentation Conference. 2016, TUPB010. DOI: $10.18429 /$ JACoW-IBIC2015-TUPB010.

[57] M. Sami Fadali and Antonio Visioli. Digital Control Engineering. 3rd ed. Academic Press, Bostony, 2009. ISBN: 9780128144336.

[58] H. H. Gutbrod. "FAIR baseline technical reports". In: GSI technical report (2006). 
[59] P. Spiller. "Status of the FAIR synchrotron projects: SIS18 upgrade and SIS100". In: Proceedings of the $5^{\text {th }}$ International Particle Accelerator Conference (IPAC14), Dresden, Germany 04 (2014), p. 1857.

[60] URL: https://www.gsi.de/forschungbeschleuniger/fair.htm.

[61] B. Franczak. "SIS parameter list". In: GSI-SIS-TN/87-13 (1987).

[62] K. Blasche et al. "High intensity Uranium operation in SIS18". In: 9 ${ }^{\text {th }}$ European Particle Accelerator Conference (EPAC 2004). July 2004.

[63] H. Grote et al. "Computer code MAD-X: user's reference manual". In: European Organization for Nuclear Research (2016).

[64] Frank Hinterberger. Physik der Teilchenbeschleuniger und Ionenoptic. Single Parcile Dynamics. Springer, 1997. ISBN: 978-3-540-75281-3.

[65] Sandira Gayadeen, Mark Heron, and Guenther Rehm. "Uncertainty Modelling of Response Matrix". In: $15^{\text {th }}$ International Conference on Accelerator and Large Experimental Physics Control Systems. 2015, MOPGF178. DoI: 10.18429/JACoWICALEPCS2015-MOPGF178.

[66] Philips J.Davis. Circulant matrices. AMS Chelsea publishing, 1994. ISBN: 978-0-82189165-0.

[67] Kamisetty Ramam Rao and Patrick C. Yip. The Transform and Data Compression Handbook. 1st ed. CRC Press, 2001. ISBN: 9781315220529. URL: https://doi.org/10. $1201 / 9781315220529$

[68] Paolo Massioni and Michel Verhaegen. "Subspace identification of circulant systems". In: Automatica 44.11 (2008), pp. 2825-2833. ISSN: 0005-1098. DOI: https://doi.org/10. 1016/j.automatica.2008.04.014. URL: http://www.sciencedirect.com/science/article/ pii/S0005109808002690.

[69] R. N. Bracewell. "Aspects of the Hartley transform". In: Proceedings of the IEEE 82.3 (1994), pp. 381-387. DOI: 10.1109/5.272142.

[70] Herbert Karner, Josef Schneid, and Christoph W Ueberhuber. "Spectral decomposition of real circulant matrices". In: Linear Algebra and its Applications 367 (2003), pp. 301311. ISSN: 0024-3795. DOI: https://doi.org/10.1016/S0024-3795(02)00664-X. URL: https://www.sciencedirect.com/science/article/pii/S002437950200664X.

[71] Arnold Neumaier. "Solving Ill-Conditioned and Singular Linear Systems: A Tutorial on Regularization". In: SIAM Review 40.3 (1998), pp. 636-666. DOI: $10.1137 /$ S0036144597321909. URL: https://doi.org/10.1137/S0036144597321909.

[72] Alan Edelman. "Eigenvalues and Condition Numbers of Random Matrices". 1988, pp. 543-560. DOI: 10.1137/0609045. URL: https://doi.org/10.1137/0609045.

[73] Moody T. Chu and Robert J. Plemmons. "Real-Valued, Low Rank, Circulant Approximation". In: SIAM Journal on Matrix Analysis and Applications 24.3 (2003), pp. 645-659. DOI: 10.1137 /S0895479801383166. URL: https://doi.org/10.1137/ S0895479801383166. 
[74] Ali R. Amir-Moéz and Chandler Davis. "Generalized Frobenius inner products". In: Math. Ann. 141, 107-112 (1960).

[75] M. Munoz and D. Einfeld. "Lattice and beam dynamics of the ALBA storage ring". In: ICFA Beam Dyn. Newslett. 44 (2007).

[76] Lin Liu, Ximenes Resende, and Fernando de Sá. "A New Optics for Sirius". In: $7^{\text {th }}$ International Particle Accelerator Conference. 2016, THPMR013. DOI: 10.18429 , JACoW-IPAC2016-THPMR013.

[77] Yuke Tian et al. "NSLS-II Fast Orbit Feedback System". In: $15^{\text {th }}$ International Conference on Accelerator and Large Experimental Physics Control Systems. 2015, MOC3O05. DOI: 10.18429/JACoW-ICALEPCS2015-MOC3O05.

[78] Alan Frieze, Ravi Kannan, and Santosh Vempala. "Fast Monte-Carlo Algorithms for Finding Low-Rank Approximations". In: J. ACM 51.6 (Nov. 2004), pp. 1025-1041. ISSN: 0004-5411. DOI: $10.1145 / 1039488.1039494$. URL: https://doi.org/10.1145/ 1039488.1039494.

[79] Sajjad Hussain Mirza et al. "Closed orbit correction at synchrotrons for symmetric and near-symmetric lattices". In: Phys. Rev. Accel. Beams 22 (7 July 2019), p. 072804. DOI: 10.1103/PhysRevAccelBeams.22.072804. uRL: https://link.aps.org/doi/10.1103/ PhysRevAccelBeams.22.072804.

[80] Jürgen Pfingstner and Jochem Snuverink. Analytic robust stability analysis of SVD orbit feedback. Tech. rep. CERN-OPEN-2012-019. CLIC-Note-946. Geneva: CERN, July 2012. URL: https://cds.cern.ch/record/1472966

[81] Sajjad Hussain Mirza et al. "Effect of Model Errors on the Closed Orbit Correction at the SIS18 Synchrotron of GSI". In: J. Phys. Conf. Ser. 1067.7 (2018), p. 072035. DOI: 10.18429/JACoW-IPAC2018-WEPAK003.

[82] Eberhardt M. et al. "Measurement and correction of the longitudinal and transverse tune during the fast energy ramp at ELSA". In: Proc. IPAC'10, Kyoto, Japan, MOPD085. (2010).

[83] Michael Green and David J. N. Limebeer. Linear Robust Control. chapter 3. USA: Prentice-Hall, Inc., 1994. ISBN: 0131022784.

[84] P.D. Lax and P. Lax. Functional Analysis. Pure and Applied Mathematics: A Wiley Series of Texts, Monographs and Tracts. Wiley, 2002. ISBN: 9780471556046.

[85] B. Zotter. "The Q-shift of off-center particle beams in elliptic vacuum chambers". In: Nuclear Instruments and Methods in Physics Research Section A: Accelerators, Spectrometers, Detectors and Associated Equipment 129 (1975), pp. 377-395.

[86] Karlheinz Schindl. "Space charge". In: (2006). DOI: 10.5170/CERN-2006-002.305. URL: https://cds.cern.ch/record/941316.

[87] M. Bei. private communication.

[88] Sajjad Hussain Mirza et al. "Performance of the closed orbit feedback systems with spatial model mismatch". In: Phys. Rev. Accel. Beams 23 (7 July 2020), p. 072801. 
DOI: 10.1103/PhysRevAccelBeams.23.072801. URL: https://link.aps.org/doi/10.1103/ PhysRevAccelBeams.23.072801.

[89] URL: https://www.i-tech.si/products/libera-hadron/.

[90] Dejan Tinta. "Closed Orbit Feedback application for Libera Hadron: user manual". In: Instrumentation Technologies, Velika Pot 22 SI-5250 Solkan, Slovenia (2020).

[91] Rahul Singh et al. "First Beam-based Test of Fast Closed Orbit Feedback System at GSI SIS18". In: $8^{\text {th }}$ International Beam Instrumentation Conference. 2019, MOPP027. DOI: $10.18429 / \mathrm{JACoW}-\mathrm{IBIC} 2019-\mathrm{MOPP} 027$.

[92] R. Yates. "Fixed-Point Arithmetic: An Introduction". In: Technical Reference. 2013. URL: www.digitalsignallabs.com/fp.pdf.

[93] B. Franczak. "SIS18 parameter list". In: GSI Technical Parameters (1987). URL: https://www.gsi.de/en/work/accelerator_operations/accelerators / heavy_ion_ synchrotron_sis18/technical_parameters.htm.

[94] D. Schupp, A. Döring, and D. Rodomonti. "Technical Note of Adaptive Control Unit (ACU) for FAIR Power Converters". In: GSI technical Note, F-TN-PC-04e (2006).

[95] R. Singh, P. Kowina, and K. Lang. "Recent improvements in TOPOS". In: GSI technical report (2015).

[96] I. Kempf et al. "Symmetry Exploitation in Orbit Feedback Systems of Synchrotrons for Computational Efficiency". In: IEEE Transactions on Nuclear Science (2021), pp. 1-1. DOI: $10.1109 / \mathrm{TNS} .2021 .3052553$.

[97] D. Beck et al. "The General Machine Timing System for FAIR and GSI". In: GSI Internal Document (2013).

[98] Tomasz Włostowski. "Precise time and frequency transfer in a White Rabbit network". Master thesis, Warsaw University of Technology, 2011.

[99] T. Hoffmann. "FESA - The front-end software architecture at FAIR". In: Proceedings of PCaPAC08, Ljubljana, Slovenia (2008), WEP007.

[100] Derek Schupp. "Universal Serial Interface verstehen (USI 1.1)". In: GSI internal document (2019). URL: https://docplayer.org/199273793-Universal-serial-interfaceverstehen-usi-1-1.html. 


\section{APPENDIX}




\section{Appendix A}

\section{A.1 GMT of FAIR}

Every component hardware used for control or measurement of the beam parameters in FAIR/GSI is attached to a Front End Controller (FEC) computer. A synchronization between the triggers and the actions of FECs is the key requirement for the successful operation of any accelerator facility. This requires a timing system that can handle machine cycles (injection to extraction) from milliseconds up to the order of several seconds for ramping synchrotrons and up to several hours for storage rings. The task for such a timing system is the real time decisions for the execution of predefined sequence of actions.

The General Machine Timing System (GMT) [97] of FAIR responsible for timely execution of the actions of FECs by sending timing messages through the timing network, is a component of the accelerator control system, shown schematically in Fig. A.1. The GMT is contained in the lowest of the three layers of the accelerator control system, the so-called Equipment Layer where two sub-systems of the GMT in the overall context of the accelerator control system are present i.e. a Timing Master and a Timing Network. Timing master is a logical device and receives a schedule for the operation of the FAIR accelerator complex from the Settings Management (situated in the middle layer). The timing master schedules actions of the Equipment connected to the Front-end Controllers (FECs) for timely execution by sending so-called timing messages through the timing network (shown as green lines in Fig. A.1. GMT is synchronized primarily by the GPS Disciplined Oscillator (GPSDO) and is complemented by the Bunch phase Timing System (BuTiS), which is a high-precision clock distribution system mainly targeted at the radio-frequency systems of the accelerator. GMT is implemented using White Rabbit (WR) 98 which is a protocol developed by CERN, GSI and other partners for synchronizing nodes in a packet-based network. It combines Gigabit-Ethernet, IEEE1588-2008 (PTP), precise knowledge of the link delay and Synchronous Ethernet. Time synchronization is achieved by adjusting the clock phase (125 MHz carrier) and offset (Coordinated Universal Time - UTC, or International Atomic Time - TAI) of all network nodes to that of a common grand master clock. It has been demonstrated in 2012, that sub-nanosecond synchronization with a jitter in the hundreds of picoseconds range is achieved over distances of a few kilometers and across dedicated WR network switches.

\section{A.2 Modules of Libera Hadron}

Figure A.2 shows the picture of Libera Hadron (top) and the block diagram for its modules (bottom) in the order of signal flow from BPMs to the corrector magnets. Since each Libera Hadron is equipped with four HBPM modules, a total of 3 Libera Hadrons are used for a total of 12 BPMs forming the global COFB system of SIS18. Out of 12 corrector magnets, 8 are connected to one and 4 are connected to another Libera Hadron. Each Libera contains 


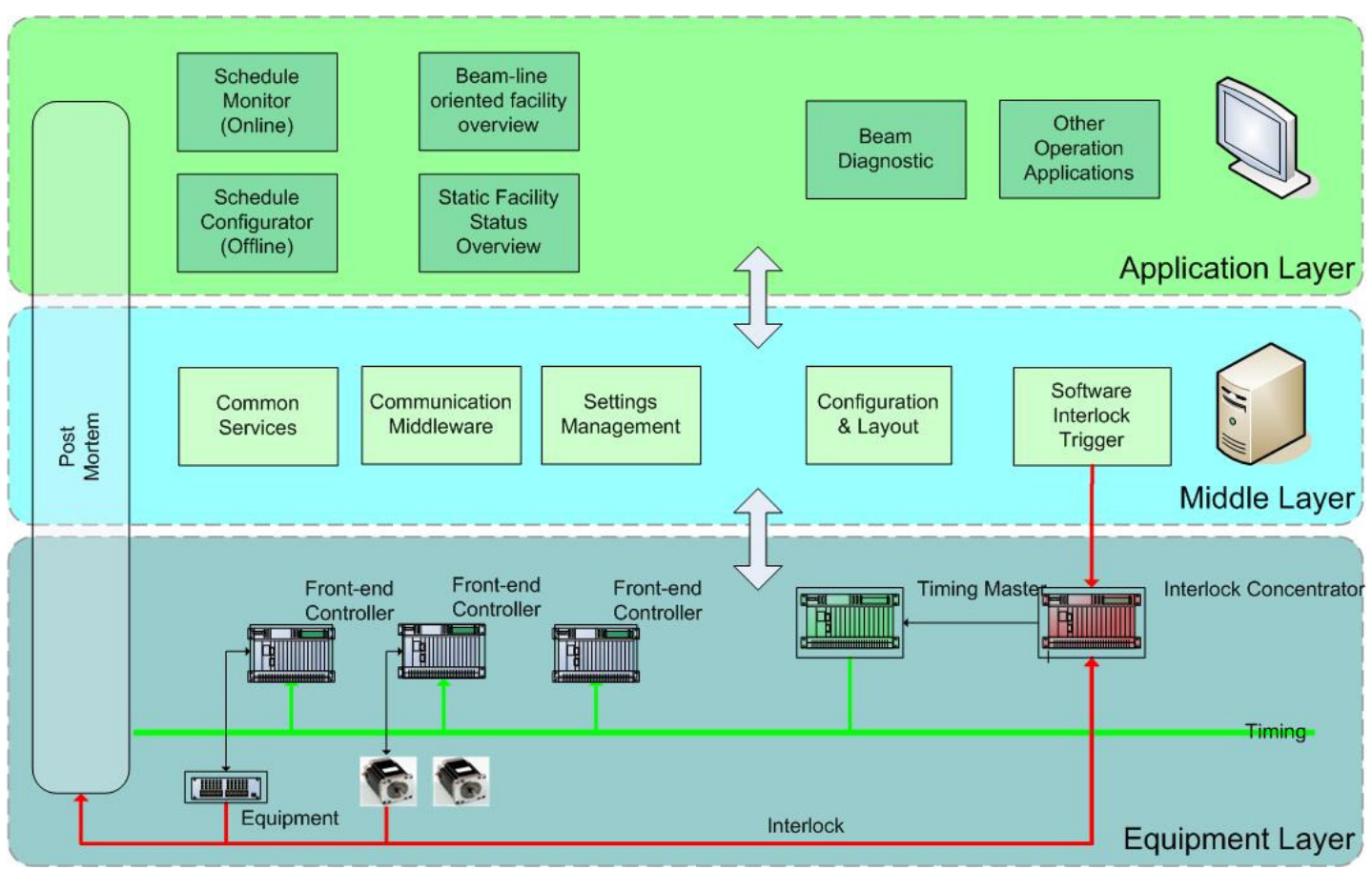

Figure A.1 Architecture Overview of the Accelerator Control System. Picture copied as it is from [97].

a board computer called Inter Connection Board (ICB) with a Measurement and Control Interface (MCI) for user's communication with all the modules. The modules are connected over a backplane which provides several control and communication channels. Further details of each module is discussed below.

\section{A.2.1 FRTN module}

The main components of the timing system are the master, the network and the receiver nodes 97. Here we shall focus on the third component which is the interface of an FEC towards the timing system and is called FAIR Timing Receiver Node (FTRN). FTRN module is embedded in the Libera Hadron and is responsible for the synchronization between the external triggers and the actions of Libera Hadron. Each FTRN is configured by third party software, typically Front-end System Architecture (FESA) [99], to execute a set of actions known in advance. These actions are scheduled for execution by the data master, which broadcasts commands containing absolute timestamps (such as Temps Atomique International - TAI) denoting the time of action execution and references to an action. When a broadcast command is received, FTRNs enqueue the designated action to be executed locally at the specified time. FTRNs have the following features:

- Clock and time synchronization with a clock master according to the WR specifications.

- Ability to record arrival time of a digital pulse (e.g. LVTTL). 

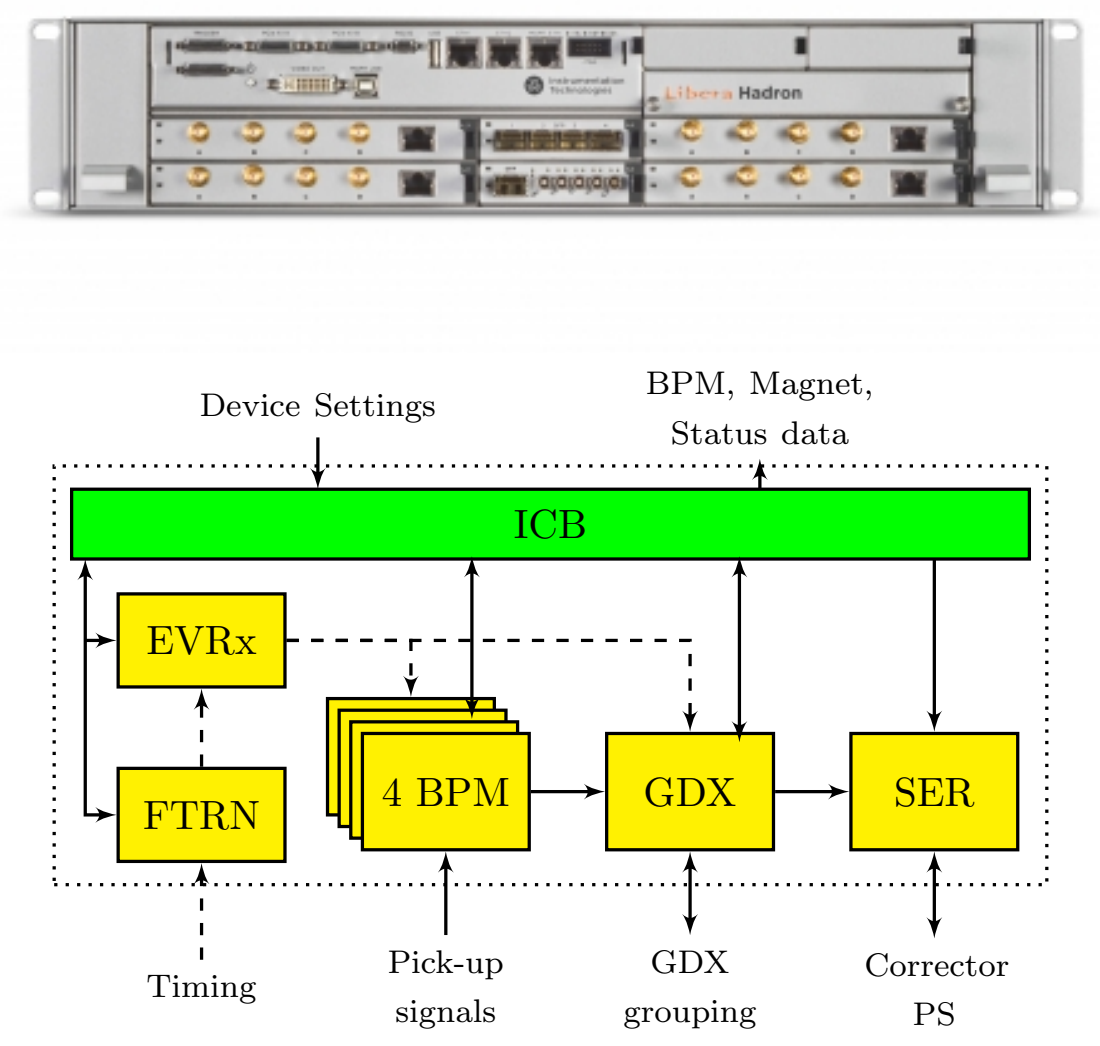

Figure A.2 Libera Hadron hardware layout. For abbreviations please see the text below.

- Ability to generate a digital pulse or a pulse sequence at a given time.

- Ability to generate clock signals with a given frequency and phase.

- Ability to generate specified interrupts at given times.

The FTRNs are connected via optical Small Form-factor Pluggable (SFP) transceiver modules to the White Rabbit (WR) timing network. WR synchronizes the internal clocks of all FTRNs in sub-ns precision and distributes timing information. In the GMT, an accelerator cycle is divided into several beam processes where the point of time when a beam process will start is exactly known. This information is used to program the FTRNs with upcoming events in advance - like setting an alarm clock - so that trigger actions can be performed on all FECs synchronously.

\section{A.2.2 EVRx module}

For the COFB system, FTRN uses two trigger outputs which are connected with Libera's EVRx module. The EvRx module (see Fig. A.3) receives the optical signal through the Small Form-factor Pluggable (SFP) transceiver and identifies and extracts the 16-bit event 


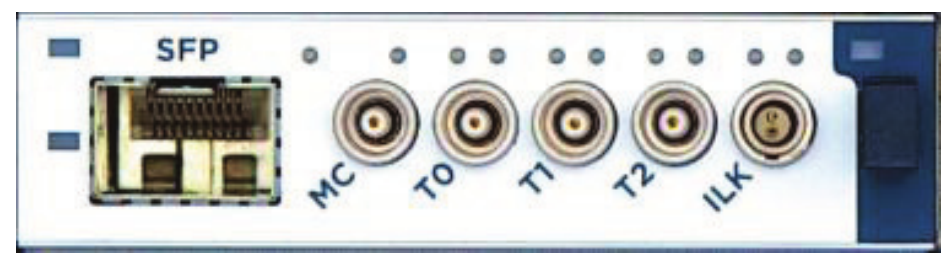

Figure A.3 EVRx module $|90|$.

code. Once the code has been extracted, the module decodes the event identification code and triggers specific functions at low latency. One trigger is used to start the specific digital signal processing (DSP) functions in HBPM modules (described in next subsection) such as position measurement and the other one is used to start the feedback system, independently. In addition, FTRN generates a continuous $10 \mathrm{MHz}$ clock signal which is connected via front panel output to the EVRx's front panel Machine Clock (MC) input. The EVRx has a Phase Locked Loop (PLL) which uses the MC input to generate the $250 \mathrm{MHz}$ clock signal for the HBPM modules so that sampling of all BPM pick-up signals of an accelerator is performed synchronously.

\section{A.2.3 HBPM module}

Each HBPM module has four input channels for the signals from four plates of each BPM and digitizes the signals at $250 \mathrm{MSa} / \mathrm{s}$ (based upon EVRx clock frequency) representing into 16-bit values referred to as the raw ADC data. The raw data is processed into the bunch-by-bunch data (at the bunch revolution frequency), Fast Acquisition (FA) (at $10 \mathrm{kHz}$ ) and Slow Acquisition (SA) (at $100 \mathrm{~Hz}$ ) streams in the FPGA. The raw ADC and position data, along with accompanying timing and status data is stored into dedicated buffers in HBPM module, according to the received start/stop trigger events. Dedicated 2 GB buffer for raw ADC data enables data acquisition with maximum acquisition length of $268 \mathrm{M}$ samples available as data on demand. Similarly, the bunch-by-bunch data is stored in another dedicated 2 GB DDR RAM buffer. Presently three position calculation algorithms options are available, "bbb" [53 or Narrow-Band Analysis "nba" algorithm and a new fit based position calculation algorithm [55, 56]. The FA data stream from all 4 HBPM modules is transferred synchronously to the GDX module of each Libera hadron.

\section{A.2.4 GDX module}

Gigabit Data eXchange (GDX) module offers fast $(6.5 \mathrm{Gbit} / \mathrm{s})$ links (SFP connectors) with GDX modules of the other Libera modules through which the orbit data is transferred at 10 $\mathrm{kHz}$ rate in hard real-time. The local FA data from the BPM modules is obtained through low-voltage differential signaling (LVDS) data channels on the Libera back-plane (Fig.A.4). The heart of the GDX is an FPGA where the feedback algorithm is implemented. 


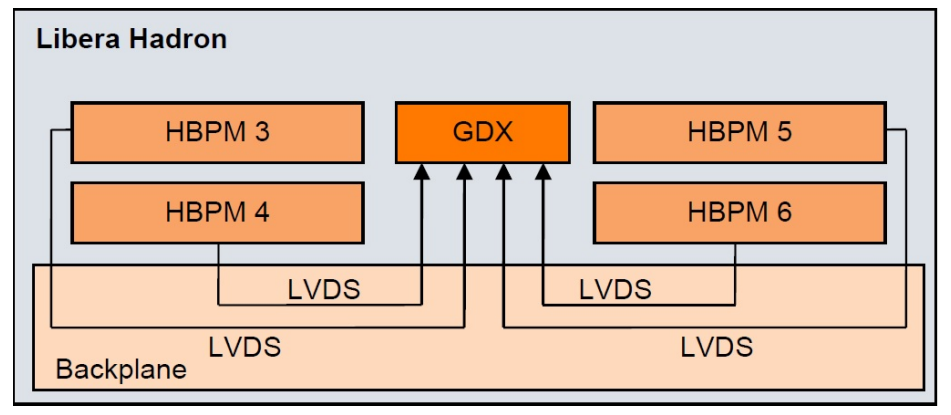

Figure A.4 Local data concentration from HBPM modules to GDX module 90 .

\section{A.2.5 SER module}

The SER module builds the interface between GDX module of Libera Hadron and the adaptive control unit (ACU) which is the modular control system of the corrector magnet power converters developed at GSI. SER provides eight RJ-45 ports where each one can be used for a point-to-point connection with an ACU. Data transmission is done over these ports by the Universal Serial Interface (USI) [100]. USI was developed along with the ACU to interconnect different components of a power converter and includes a RS-485 based protocol for communication. The GDX module sends 8 out of 12 user-selectable 20 Bit wide magnet correction-values to the SER module of one Libera Hadron and 4 to another Libera Hadron with $10 \mathrm{kHz}$ data rate synchronous to the COFB execution rate. The SER module caches these corrections in a buffer. For communication via USI, the ACU always has to act as master and polls the data from the SER module asynchronously. In our setup Cat 6 (Ethernet) cables have been used on the physical layer and a maximum baud rate (waveform changes per second) of $16 \mathrm{MBit} / \mathrm{s}$ has been reached for a cable length of $30 \mathrm{~m}$. At our chosen baud rate and with total protocol overhead, the maximum polling rate is then $133 \mathrm{kHz}$.

\section{A.2.6 COFB validity state machine}

The Validity State Machine (VSM) is responsible for controlling (stopping \& resuming) the COFB feedback in the following way: The VSM stops the COFB system,

- Whenever no bunch-by-bunch data is available within one period of orbit correction.

- Whenever an overload or underload occurs.

- Whenever the result of Libera Grouping is not valid (missing data in vector $\mathbf{x}$ or $\mathbf{y}$ ).

- Whenever there is a fault on the ACU side of the magnet power converters.

The user can select on software level which of these conditions shall be taken into account by the VSM. Each of these causes can be made hidden using "masks". When the VSM stops the COFB system,

- The error signal fed to the PI controller is set to zero. 
- The magnet output is either set to the integral part of the last calculated valid magnet correction value or to constant values, predefined by the user

VSM resumes the COFB system,

- Immediately after the conditions which led to stop COFB system become valid (e.g. bunches get detected), if the "Auto Resume" signal is true.

- After a manual user resume. 


\section{Appendix B}

\section{B.1 ACU of corrector power converters}

- ACU has Eight 1-pole Lemo jacks out of which 4 are digital to analogue (DAC) outputs that act as diagnostic outputs and input/output signal of both control loops. The DACs have a resolution of 14 bit and a sampling rate of up to $250 \mathrm{kSPS}$.

- Analogue to digital (ADC) modules of ACU make it possible to acquire analogue signals from the power converter and corrector magnets such as load current, for measuring and controlling purposes. The ADC module has a resolution of 18 bit and a sampling rate of up to $600 \mathrm{kSPS}$.

- The "Power Config Advanced" software is used for configuring an ACU from a personal computer. The computer is connected via USB cable to the Multifunction Module of ACU. A screenshot of the software is shown in Fig. B.2.

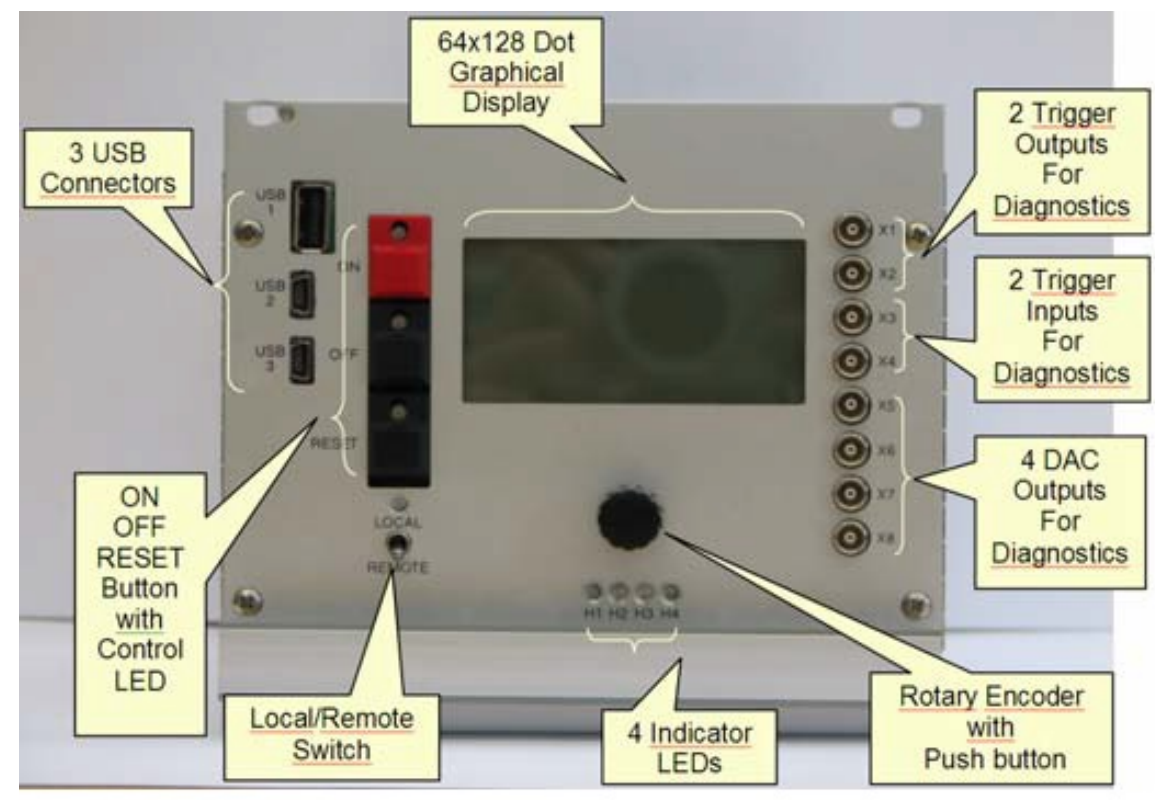

Figure B.1 Front board of Multifunction Module of ACU unit. 


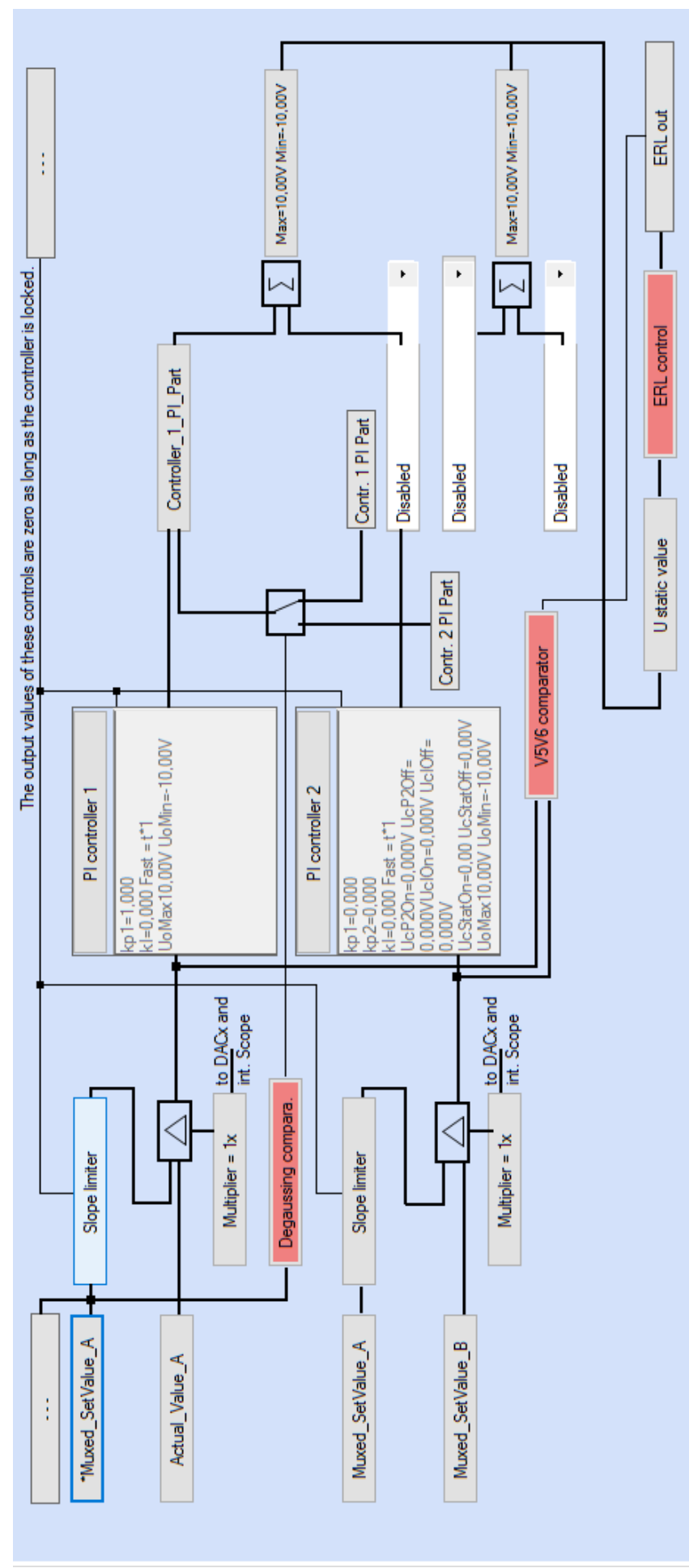

Figure B.2 A screen shot of "Power Config Advanced" software to set the controller parameters in the adaptive control units connected to the horizontal corrector power converters of SIS18. 


\section{Acknowledgement}

I am thankful with immense gratitude to my professor PD Dr. Giuliano Franchetti from Goethe University Frankfurt for his all-time support, encouragement and motivating behavior. He always stood by my side through thick and thin and rescued me in every crisis during my $\mathrm{PhD}$. His insightful comments and questions enormously improved the quality of this thesis. I also acknowledge Prof. Dr. Ulrich Ratzinger's valuable comments on the thesis as well as his support in university related matters, as member of my thesis committee.

I pay my gratitude to Dr Peter Forck for making possible my arrival to GSI by offering me the $\mathrm{PhD}$ project and getting DAAD funding with his acceptance letter. During my stay at GSI, he has been a solution to almost every problem, whether technical or management related. His dynamism, sincerity, patience, and motivation have deeply inspired me. He took proper care of my education and international projection of my work by recommending me for various courses, conferences, workshops and grants. His trust in me and his caring and helping behavior will be in my memories, forever.

I would also like to express my deepest gratitude to Dr Rahul Singh from GSI; without his contribution, the work presented in this thesis would not have been possible. His wisdom, encouragement and confidence in me pushed me farther than I thought I could go. His continuous guidance, innovative ideas and discussions and help in timely designing and conducting experiments at GSI and COSY Jülich made possible the successful and timely completion of this thesis. Along with technical support, he was always available to listen to my concerns and doubts as a friend.

My sincere thanks also go to Kevin Lang and Timo Milosic from GSI and to Aleš Bardorfer from Instrumentation Technologies, Slovenia for their support during setting up the hardware. In addition, special thanks to Horst Welker, Davide Rodomonti, Axel Doering from the power supply group of GSI for their support during the system's commissioning.

I would not like to forget the support and guidance of Prof. Harald Klingbeil from Technical University Darmstadt, particularly during first three years of my PhD.

A special thanks go to Dr David Ondreka and Dr Stefan Sorge from GSI for their support to get familiar with the machine models and parameters at the GSI synchrotrons.

I would also like to thank all of my colleagues at the Beam Instrumentation department of GSI, particularly Head of department Marcus Schwickert for his encouragement and support that led to the successful commissioning of the closed orbit feedback system at SIS18. I am specially thankful to my friends Thomas Reichert and Rainer Haseitl for their emotional support during my stay in GSI.

DAAD (Deutscher Akademischer Austauschdienst) is also highly acknowledged for the financial support of my living expenses during doctoral research work (Reference No. 91605207). 